This repor wis done with support from the Department of Energy. A.cy ennelusions or opinions expressed in this report represent solely those of the author(s) and nol necessarily those of The Regents of the University of California, the Latrence Betkeley Labosalory of the Depariment of Energy.

Reference to a company or product name does not imply approval of recommendation of the product by the University of California or the U.S. Deparament of Energy to the exclusion of others that may be suitable. 


\title{
DISCLAIMER
}

This repor was prepared as an account of work sponsored hy an agency of the United States Government. Nejther the United States Covernment nor any agency thereof, nor any of their employees, makes any warranty. express or implicd, or assumes any legal hability or responsibility for the accuracy, compieteness, or usefuiness of any information, apparatus, product. or process disclosed, or represents that its use would not infringe privately owned rights. Reference herein to any specifte commercial produc;. process, or service hy trade name, trademark. manufacturer, or otherwise dies not necessarily constilute or im 1 l jis endorsement, recommendation, or favoring by the Inited Slates Ginvernment or ant agency thereof. The views and opinions of authors expressed herein do nut necessistly state or reflect those of the United States Government or any agency therwif

\section{Measurement of the Nucleon Structure Function Using High Energy Muons}

\author{
Peter Danie] Meyers \\ Ph.D. Thesis \\ Lawrence Berkeley Laboratory \\ University of Californja \\ Berkeley, CA 94720 \\ December 2, 1983
}

\begin{abstract}
We have measured the inclusive deep inelastic scattering of muons on nucleons in iron using beams of 93 and $215 \mathrm{GeV}$ muons. To perform this measurement, we have built and operated the Multimuon Spectrometer (MMS) in the muon beam at Fermilab. The MMS is a magnetized iron target/spectrometer/calorimeter which provides $5.61 \mathrm{~kg} / \mathrm{cm}^{2}$ of target. $9 c_{c}^{-}$ momentum resolution on scattered muons. and a direct measure of total hadronic energy witb resolution $\sigma_{\nu}=1.4 \sqrt{l^{\prime}(\mathrm{GeV})}$. In the distributed target, the average beam energies at the interaction are 88.0 and $209 \mathrm{GeV}^{-}$. Lsing the known form of the radiatively-corrected electromagnetic cross section. we extract the structure function $F_{2}\left(x, Q^{2}\right)$ with a typical precision of $2 \%$ over the range $5<Q^{2}<200 \mathrm{GeV}^{2} / \mathrm{c}^{2}$. We compare our measurements to the predictions of lowest order quantum chromodynamics ( $Q C D$ ) and find a best fit ralue of the $Q C D$ scale parameter $\Lambda_{\text {LO }}=230 \pm 40^{\text {stat }} \pm 80^{\text {syst }} \mathrm{MeV} / \mathrm{c}$. assuming $R=0$ and without applying Fermi motion corrections. Comparing the cross sections at the two beam energies, we measure $R=-0.06 \pm 0.06^{\text {rtat }} \pm 0.11^{\text {syst }}$. Our measurements sbow qualitative agreement with $Q C D$, but quantitative comparison is hampered by phenomenological uncertainties. The experimen1al situation is quite good. with substantial agreement between our measurements and those of others.
\end{abstract}

\section{RITISE}

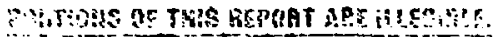

$\because$ : $;$ :

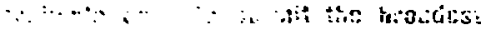

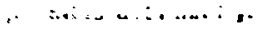




\section{Contents}

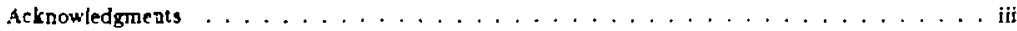

Chapter 1. Probing the Structure of Matter $\ldots \ldots \ldots \ldots \ldots$

Chapter 2. The Phenomenole $y$ of Deep Inelastic Scattering . . . . . . . . . . 3

2.1 Kinematics . . . . . . . . . . . . . . . . . . . . 3

2.2 Cross section and structure functions $\ldots \ldots \ldots \ldots \ldots \ldots$

2.3 Virtual photons . . . . . . . . . . . . . . . . .

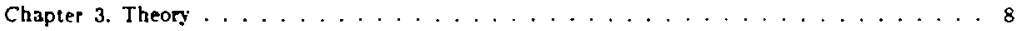

3.1 The parton model . . . . . . . . . . . . . . . . . . . 8

3.2 Quantum chromodynamics . . . . . . . . . . . . . . . . . . . 10

3.3 Grand unifled theories . . . . . . . . . . . . . . . . . . . . . . . .

3.4 Theoretical summary and experimental program . . . . . . . . . . . . . . 19

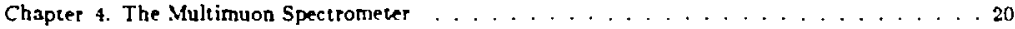

4.I Design constraints . . . . . . . . . . . . . . . . . . . . 20

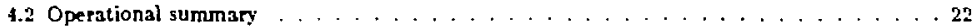

4.3 The muon beam . . . . . . . . . . . . . . . . . . . . . . . . 22

4.4 Target and magnet. . . . . . . . . . . . . . . . . 25

4.5 Trigger . . . . . . . . . . . . . . . . . . . . . . . . . . . . .

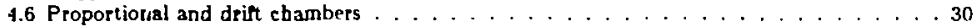

4.7 Calorimeter . . . . . . . . . . . . . . . . . . . . . . . . .

Chapter 5. Dats Taking and Data Scts . . . . . . . . . . . . . 39

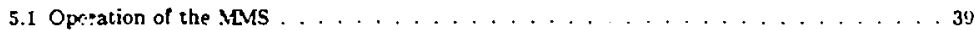

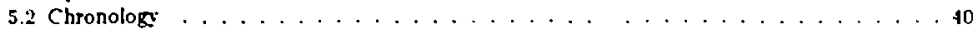

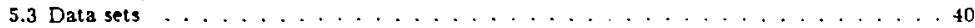

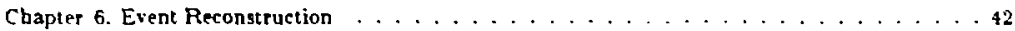

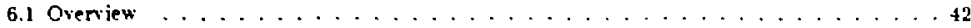

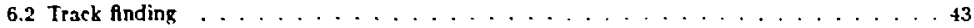

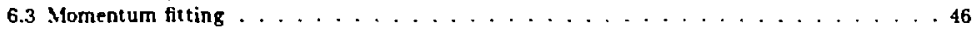

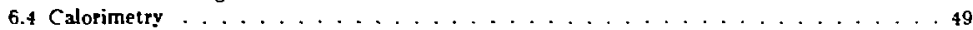

6.5 Perfo:mance . . . . . . . . . . . . . . . . . . . 49 
Chapter $i$. Alignment and $\mathrm{Calibration} \ldots \ldots \ldots \ldots \ldots \ldots \ldots$

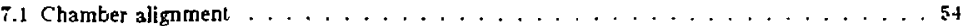

7.2 Magnetic fleld integrals . . . . . . . . . . . . . . . 55

i.3 Calorirneter calibration $\ldots \ldots \ldots \ldots \ldots \ldots$

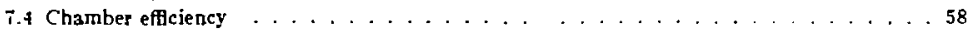

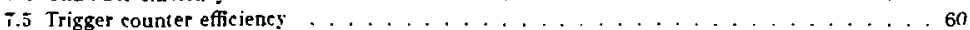

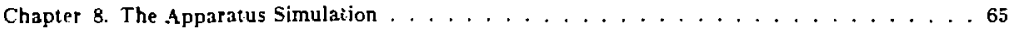

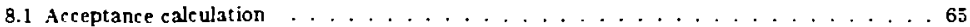

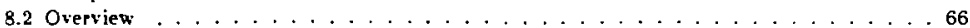

8.3 Beam and target . . . . . . . . . . . . . . . . . . . . . . . . . .

8. Event generation . . . . . . . . . . . . . . . . . . . . . . . . .

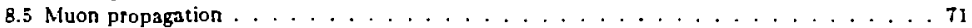

8.6 Data simulation . . . . . . . . . . . . . . . . . . 75

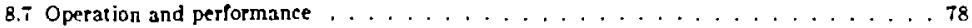

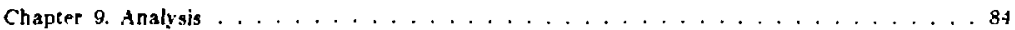

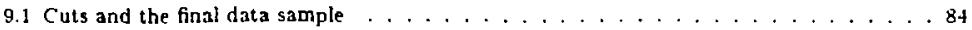

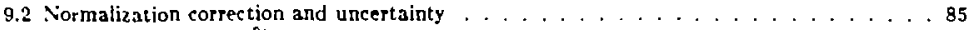

9.3 Estraction of $F_{2}\left(x, Q^{2}\right) \ldots \ldots \ldots 5$

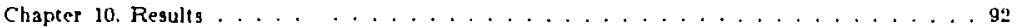

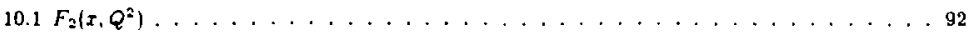

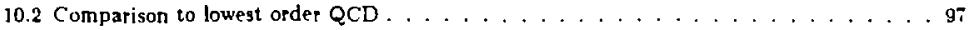

10.3 Systematic uncertainties . . . . . . . . . . . . . . . . . . 98

10.4 Pheromenological uncertainties . . . . . . . . . . . . . . 106

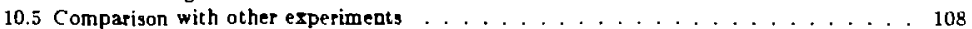

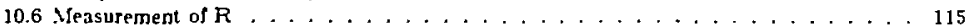

10.7 Search for a $\mu^{+-, 2}-$ ssymmetry . . . . . . . . . . . . . . 118

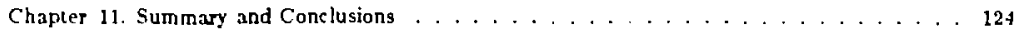

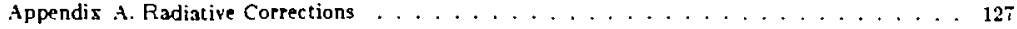

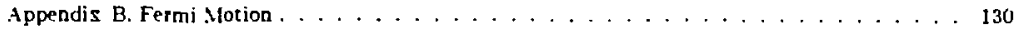

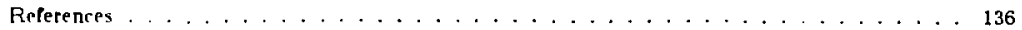




\title{
Acknowledgments
}

\author{
Q: How mang Berketer phrsics greduace students does it leze ro \\ chagge light bolb? \\ A: Just one, but it tales bim efght years.
}

Fungy joke, c. 1970

This docament is the closing chapter of an effort that occupied many people tor the better part of a decade. Fermilab Experiment 203/391 was designed, built, and operated by a collaboration of physicists from the Lawrence Berkeley Laboratory. Fermilab, and Princeton Unirersity. The members of this group were A Clark. George Gollin, Karl Johnson, Rol Johnson, Roy Kerth. Stew Loken, Tom Markjewicz. Craig Moore, Marshall Mugge, Bob Shafer, Frank Shoemaker, Wes Smith, Mark Strovink. Pam Surko, Bill Wenzel, and me. At LBL we were assisted by the technical skills of Fred Goozen, Tom Webet. Tim Nuzum. and Duck (aka Elaine. aka kay) Lucas. Also instrumental were Leslie Bauman and others of the Fermilab staff. Within our group. Marshall Mugge and Stew Loken worked with me on the analysis of our deep inelastic scattering data, which forms the basis of this thesis.

The hardships infolved in a small group (about $1 / 5$ the size of a typical one for an effort of this magnitude) doing an experiment far from home should not be forgotten. The joys of working at Fermilab are related in a cycle of poems written by George Gollin and myself which is not included in this werk. Suffee it to say that somebody had to shovel the manure out of that barn. It is this sort of direct contact with unvarnished reality that distinguishes experimental from theoretical physics.

Besides the direct collaboration with Marsball and Stew, I hare been aided by many people in this endeavor. Mark Strovink advised me on many points of physics; Tom Markiewicz. fellow student, roommate, and friend, advised me on everything else. Janet Remer. Pat Bronnenberg. and Edgar Whipple are responsibie for whatever is attractive in the appearance of the thesis. The experiment itself was funded by the Director, Office of Energy Research. OAfe of Bigh Energy and Nuclear Pbysics, High Energ Pbysics Division of the Lnited States Department of Energy under Contract Nos. DE-AC03-76SF00098. DE-AC02-i6CH03000, and DE-ACO2-iCER030-2. I was supported by a National Science Foundation Graduate Fellowship during the years isis-77. Of greater importance was the emotional support I received from family and friends, who must have wondered what was going on all this time, and from Marjorie Shapiro. who knew.

I worked on this experiment for $8 \frac{1}{2}$ years. On the whole I'm quite proud of the results, something I could not hove said 3 year ago. On the ot ber hand, much can happen in $8 \frac{1}{2}$ years, and the rest of the world has not been standing still. Want of $\mathrm{my}$ contemporaries have establisbed families and careers. Against this candle of decency, the world has hurled a $\$ 000000000000$ 
hurricane of military spending. Although I am grateful for the unqualifled shelter provided by the University of California, my step into the world is oferdue. I take that step now with some trepidation. but secure in the knowledge that, whatever the result, the cause will aot be lack of scbooling. 
To my Mother and Father, who never made me become a real doctor. 


\section{Chapter 1 \\ Probing the Structure of Matter}

This is the las polato I bave oug in my life.

E. Rutberford, 1894

When a human being wants to see an object directly, she shines a light un it. The structure of the object is revealed by the pattern of light scattered to the eye. Perbaps because we are naturally equipped io interpret such information. the estension of this process to the subatomic realm bas been consistently successful in exposing the successive layers in the structure of matter. To make this kind of direct obserration, the probes employed must meet several requirements. They must be pointlike: their structure must not complicate the measurement and their warelength must be small enough. their moment um large enough. to probe the lengt scale of interest. They must be able to penetrate the structure under investigation down to the length scale of interest. For charged probes this requires a sufficiently large energy. Finally. the interaction of the probe with the target must be simple enough to allow an interpretation of the scattering in terms of the target's structure. Since the tirst decade of tbis century. the electromagnetic scattering of pointlike particles bas sbown us the structure of atoms and the (unexpected) existence of the at omic nucleus. the struct ure of the at omic nucleus and its size. and the structure of the aucleon and the (unexpected) esistence of partons. later to be identifed with quarks. The ezperiment recorded bere continues this investigation. using the principle erseatially unchanged since 1909 , but at a scale $10^{5}$ times smaller.

At the turn of the century. the current model of the atom nas tbat of J.J. Thomson. ${ }^{1}$ It postulated an array of ligbt, negat/vely charged electrons embedded in a uniform ball of positive charge. This model was able to explain the small but measured deflections of cbarged particles by thin lasers of matter as tbe result of many gentle scattcrs." In 1909, however. Geiger and Marsden ${ }^{3}$ working in Ernest Rutberford's laborat ory at Manchester. found that there was a small ( of platinum. Even this small rate of large angle scattering a ss impossible in Thom $\operatorname{con}^{\mathrm{s}} \mathrm{s}$ model. cither through a sucession of small acatters of through a ringle large oufe. The dituse nature of the charge distribution did not produce an electric field of sufficient intensity to tura tbe a particles through large angles. Rutherford ${ }^{+}$realized that the observed large-angle sratters could be produced only by the concentration of one siga of charge at the center of the atom. The known properties of electromagnetism allowed Rutherford in make detailed predictions of scattering probahilits vs. angle, aromic number, and velocity for his model with a pointlike nucleus. The predictions mere confirmed by the subsequent experiments of Geiger and Marsden."

Geiger and Marsden's a particles could onls penetrate 10 xit hin $3000 \mathrm{fm}=3 \times 10^{-10} \mathrm{~cm}$ of the center of a beary atom. Direct measurement of nuclear sizes $(\approx 5 \mathrm{fm} / \mathrm{had}$ to $\mathrm{xat}$ for the 
bigher energies provided by accelerators. Measurements using beams of high energr ( $\approx 500 \mathrm{MeV}$ ) electrons ${ }^{\theta}$ showed suppression of large augle elastic scattering from nuclei. The interpretation was simply the inverse of Rutberford's concerning the atom: the nucleus was a flailesized distribution of cbarge. The suppression of the scattering cross section is described by a "form factor" $F(q)$. where $q$ is the momentum transferred to the target. a quantits that increases as the scattering angle increases. If the energy transfer in the collision is small. the form factor is the Fourier transform of the spatial distribution of rharge. An extended charge distribution leads to an $F(q)$ less than one and generally decreasing witb increasing $1 \mathbf{q}$.

The proton also showed a non-unit form factor, indicating a charge radius of $\approx 0 . i \mathrm{fm}$ This, however, represented the resolution limit of elastic electron-nucleon scattering. Demanding an elastic scatter in effect requires a moment un uncertaints in the hadron finat state of less than a pion mass. The uncertainty principle then restricts spatial resolution to approsimately $1 \mathrm{fm}$ at large moment um transfers for which the recoiling nucleon is relativistic. To reap the benefits of bigher moment um transfer (shorter waveleng1 $b$, it is nec 'ary to relax the elastic requirement and consider inelastic scattering. Early stiz as of inelastic eN scattering were directed primarily at the resonance region near the elastic limit. ${ }^{i}$ but in 1969. when the SLAC.MIIT group first louked at the "dcep ibelastic continuum," ihes were surprised to fiad very little form-factor type suppression ai large momentum transfer, in clear contrast to the elastic cross section.

These results were immediately interpreted ${ }^{0}$ tbrough a model that would have seemed familiar to Rucherford. The proposed model post ulated a nuclean composed of poinljike charged "partons" which were assumed to interact with each other on a time scale longer than that characteristic of the scattering process. The parton model reproduced the weak moment um transfer suppression obserted in the data and also the property of scaling", predicted from other considerations by Bjorken ${ }^{10}$ and confirmed by experiment. ${ }^{5}$ For a spherically spmaetic target. the elastic scattering form factor depends only on the magnitude of the momentum transfer $|q|$. In inelastic scattering. the counterpart to the elastic form factor is the "structure function." which can depenci on botb $q^{2}$. the square of the 4 -roment um transfer. and . the energy transfer in the target rest frame. In the parton model, the structure function depends on these variables only in the dimensionless combination $x \equiv-q^{2} / 2.1 \%$ where $M$ is the wass of the target nucleon The dimensionless nat ure of this dependence is called scaling.

The partons inferred from deep inelastic scattering were alwas suspected to be the quarks that Gell-Mann ${ }^{11}$ and $Z$ weig ${ }^{12}$ bad invoked to explain the proliferating chemistry of hadrons in the wid-1960's However, the dynamical theory of quartic necessary to interpret ccattering experiments was not developed until the mid-1970's. ${ }^{13}$ By this time, continued experimental investigations. now using muons, had found $\mathrm{mmall}$ riolations of scaling ${ }^{\text {it }}$ The dyamical theory. called quant um chromodynamics (QCD). was found to contar slow (logarithmic) scaling violations due 10 the interactions of the quarks va gluons. the postulated carriers of the st rong

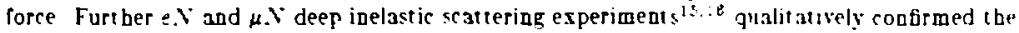
predicted pattern of scaling riolations.

Tbe experimental task at the end of the seventies was to challenge OCD quantitatively: to test its validity and if conflemed. measure its undeiermined parameters. Our axperiment ass designed and built to make such measurements by pefforming a precice determination of the nucleon structure function using the deep inelastic scattering of high energ. muons By doing so. we learn not only about the structure of the nucleon. but. if QCD is valid about the structure of the strong interaction. 
Chapter 2

\section{The Phenomenology of Deep Inelastic Scattering}

ELECTRICTY. D. The power that causes all asteral phenomena not

know $a$ to be ceused by oompther elip

Anbrose Biprce, Tbe Deril': Dictionary

\subsection{Kinematics}

The Feyman diagram for deep inclastic scattering in the lowest order of $Q E D$ is show in Fig. 2.1, together witb a summary of our kinemañical notation. To this order, the process is described as the exchange of one rirtual photon. OF.D allows us to calculate unambiguously: what bappens at the leptonic (upper) vertes. The goal of our experiment is to uncover wbat bappens in $t$ be region surrounding the badronic rertex. Simply draxing the diagram indicates an inmediate sumplification. The only lines entering the badronic vertes are those representing the satial nucleon and the virtual photon. We will be studying inclusive scatiering. $\mu . \gamma-\mu x$. $\times$ it no reference to any particular badronic final state. This means that the only relevant 4-vectors are $p$ and $q$. and the only Lorentz-invariant quantities are $q^{2}$ and $p \cdot q$ (and $p^{2}=. / 2$ ). While Isolat ing the badronic rertex is a produrtive move toward understanding the scattering process. experimentaly it is amponant to note tbat these quantities can be measured using only the initial and thal muons:

$$
\begin{aligned}
& Q^{2} \equiv-q^{2}=4 E E^{\prime} \sin ^{2} \frac{H}{\ddot{*}} \\
& l \equiv \rho q / \mu,=E-F^{\prime}
\end{aligned}
$$

xhere $E, E^{\prime}$, and ? are the intial and flna! muon energies and the muon scattering angle as measured in tbe laboratory frame t the target rest framel. Terms containing the lepton mas: bare been neglected. Anotber useful quantity is the invaint mass of the badronic final state: $H^{2}=(p+q)^{2}=M^{2}+2 W_{N}{ }^{2}-Q^{2}$. The elastic limit is $H^{2}=W_{X}^{2}$ or $Q^{2}=2 . W_{N}$. Resonances appear at fised $H^{2}$ near this limit. Figure 2.2 shows the region of the $Q^{2}-l^{2}$ plane accosible to inelastic scattering at ixed incideat enefgr $E$. 


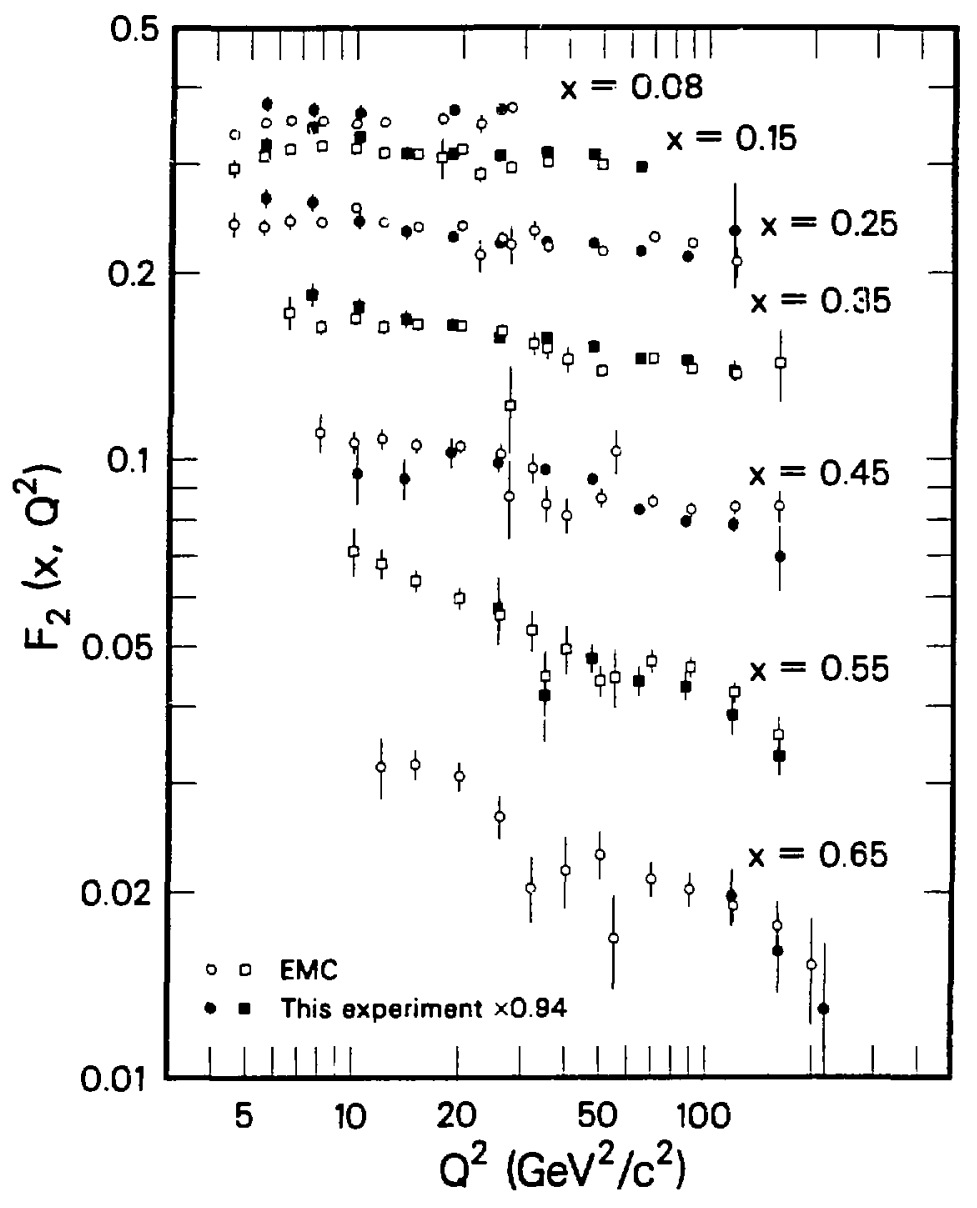

XCG $8311-4904$

Fis. 10.8. A comparison of our measured $F_{2}\left(r, Q^{2}\right)$ (mutiplied by 0.94) to the EMC's iron target measurement (Ref. $6 \pi$.). 


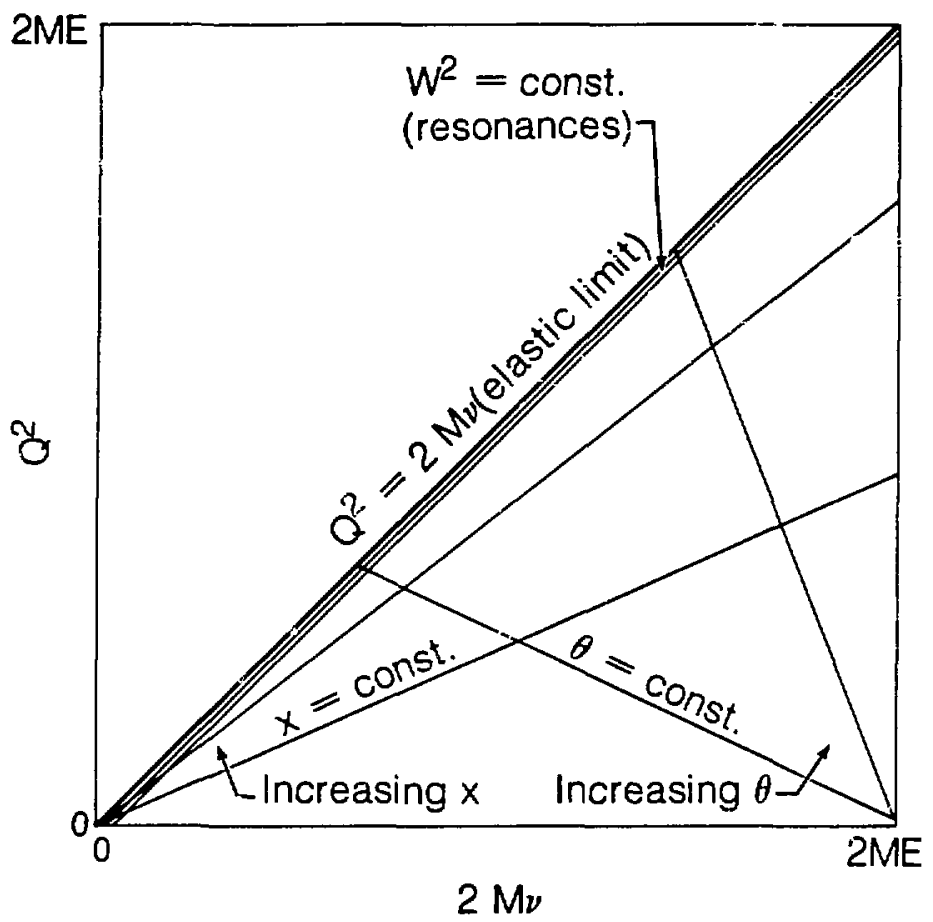

XBL B310.740

Fig. 2.2. Deep inelastic scattering kinematics. The region below the diagonal is accessible. Lines of constant $\boldsymbol{H}^{2}, x$, and $\theta$ are indicated. 
It is convenient to describe another set of variables which are $Q^{2}$ and $v$ scaled by their maximum values, neglecting lepton masses:

$$
\begin{aligned}
& x=Q^{2} / 2 M_{N} v \leq 1, \\
& y=\nu / E \leq 1, \\
& v=Q^{2} / 2 M_{N} E=x y .
\end{aligned}
$$

\subsection{Cross section and structure functions}

We can translate ${ }^{17}$ the diagram of Fig. 2.1 directly into an expression for the spinareraged inclusive cross section:

$$
\begin{aligned}
d \sigma=\frac{1}{i_{\text {rel }}}(2 \pi)^{4} \delta^{4}\left(k+p-k^{\prime}-\right. & \left.p^{\prime}\right) \frac{(4 \pi \alpha)^{2}}{Q^{4}} \frac{d^{3} \mathbf{k}^{\prime}}{(2 \pi)^{3}} \\
& \times \frac{m^{2}}{E E^{\prime}} \frac{1}{4} \sum_{\text {gpins }} \sum_{X}\left|\left(\bar{u}_{k^{\prime}} \gamma_{\mu} u_{k}\right)<X\right| J^{\mu}(0)|p>|^{2} .
\end{aligned}
$$

In this expression, $m$ is the lepton mass. $\bar{t}_{k^{\prime}} \gamma_{\mu} t_{k}$ is the known lepton electromagnetic current. and $J^{\mu}(0)$ is the anknown hadron electromagnetic current. Despite this ignorance, wuch furtber progress can be made in reducing this expression. ${ }^{18}$ We start by isolating the unknown part of the square of the matris element and defining the "badron tensor"

$$
W^{\mu \nu}=\frac{1}{2 \pi} \frac{E_{N}}{M_{N}} \sum_{X} \frac{1}{2} \sum_{\text {spins }}\left\langle p\left|J^{\mu}(0)\right| X><X\left|J^{\nu}(0)\right| p\right\rangle(2 \pi)^{4} \delta^{4}\left(p+q-p^{\prime}\right) .
$$

Lorenta covariance demands that this tensor be constructed of the available tensor quantities with scalar coeffients. Because the corresponding lepton tensor (with which the hadron tensor will be contracted) is symmetric under interchange of $\mu$ and $v$, we keep only symmetric terms:

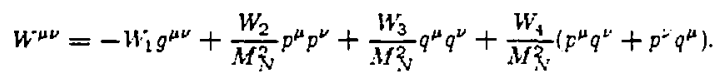

The structure functions $W_{i}$ are scalar functions and therefore depend only on $Q^{2}$ and $\nu$. Gauge invariance requires that $q_{\mu} W^{\mu \nu}=q_{\nu} W^{\mu \nu}=0$. With these constraints we can eliminate $W_{3}$ and H. leaving

$$
W^{\mu \nu}=W_{1}\left(-q^{\mu \nu}+\frac{q^{\mu} q^{\nu}}{q^{2}}\right)+\frac{W_{2}}{M}\left(p^{\mu}-\frac{p \cdot q}{q^{2}} q^{\mu}\right)\left(p^{\nu}-\frac{p \cdot q}{q^{2}} q^{\nu}\right) .
$$

Contracting this with the lepton tensor, we get a compact expression for the differential cross section:

$$
\frac{d^{2} \sigma}{d E^{\prime} d \Omega^{\prime}}=\frac{E E^{\prime}}{\pi} \frac{d^{2} \sigma}{d Q^{2} d \nu}=\frac{4 a^{2} E^{\prime 2}}{Q^{4}}\left[2 \psi_{1}\left(Q^{2} \cdot \nu\right) \sin ^{2} \frac{\theta}{2}+H_{2}\left(Q^{2}, \nu\right) \cos ^{2} \frac{\theta}{2}\right] .
$$

in terms of the tro unknown structure functions $H_{1}$ and $H_{2}$. 


\subsection{Virtual photons}

Further enlightenment comes from a complementary approach to the same process. ${ }^{10}$ We treat the incident muon as a source of virtual photons and wite the cross section as $d \sigma=\sum_{c} d \Gamma_{\ell} \sigma_{t}$, where $\Gamma_{t}$ and $\sigma_{\ell}$ are the flux and absorption cross section. respectively. for virtual photons of polarization $f$. $\tau^{*}$ sing the Feynman ruies for the reduced diagram of $\gamma^{*} X^{*} \rightarrow X$.

$$
\sigma_{\mathrm{E}}=\frac{4 \pi^{2} \alpha}{K^{*}} \dot{\epsilon}_{\mu} H^{-\mu \nu} \epsilon_{\nu}^{\prime \prime},
$$

Fhere $F^{-}$is the virtual photon flux factor.

We now apply the gauge invariance condition in the form $q^{\mu} \varepsilon_{\mu}=0$. This is easiest in the "virtual photon rest frame" (indicated with primes). where we choose the basis polarizations

$$
\begin{gathered}
\epsilon_{1}^{\prime}=(0,1,0.0), \quad \varepsilon_{2}^{\prime}=(0,0,1,0), \quad \epsilon_{3}^{\prime}=(0,0,0,1) \\
q^{\prime}=\left(\sqrt{Q^{2}}, 0.0 .0\right) .
\end{gathered}
$$

Boosting into the lab frame with the $z$-asis chosen along the virtual photon direction gives

$$
\begin{gathered}
\epsilon_{1}=\epsilon_{1}^{\prime} . \quad \epsilon_{2}=\epsilon_{2}^{\prime} . \quad \epsilon_{3}=\frac{1}{\sqrt{Q^{2}}}\left(\sqrt{Q^{2}+\iota^{2}}, 0,0, \nu\right), \\
q=\left(\nu, 0,0, \sqrt{Q^{2}+\nu^{2}}\right) .
\end{gathered}
$$

Explicitly contracting $\epsilon_{\mu} W^{\mu \nu} \varepsilon_{\nu}^{*}$ we flnd

$$
\begin{aligned}
& \sigma_{T} \equiv \frac{1}{2}\left(\sigma_{\mathrm{C}_{1}}+\sigma_{\varepsilon_{2}}\right)=\frac{4 \pi^{2} \alpha}{K} H_{1}, \\
& \sigma_{L} \equiv \sigma_{e_{3}}=\frac{4 \pi^{2} \alpha}{K}\left[H_{2}\left(1+\frac{l^{2}}{Q^{2}}\right)-H_{1}\right] .
\end{aligned}
$$

where $T$ and $L$ refer to transversely and longitudinally polarized rirtual phocons. Defining $R \equiv \sigma_{L} / \sigma_{T}$ we can eliminate $W_{1}$ in faror of $R$ in the cross section Eq. $2 . T$. The advantages of this substitution will be described in the nest section where it is show that for some cases of interest $R$ is expected to be small. For now we record the cross section in its new form.

$$
\begin{aligned}
\frac{d^{2} \sigma}{d Q^{2} d \nu} & =\frac{4 \pi \alpha^{2}}{Q^{4}}\left[1-y+\frac{y^{2}+2 H_{X} x y / E}{2(R+1)}-\frac{M_{X} x y}{2 E}\right] H_{2}\left(Q^{2}, \nu\right) \\
& \approx \frac{4 \pi \alpha^{2}}{Q^{4}}\left[1-y+\frac{y^{2}}{2(R+1)}\right] H_{2}\left(Q^{2}, \nu\right) .
\end{aligned}
$$

The approximate form comes from taking the Bjorken limit where energies $\left(E, Q^{2}, u\right) \rightarrow x$ xith $z$ and $y$ flnite. ${ }^{10}$ In the kinematic region covered by our data. making such an approsimation has a maximum effect of $<\frac{1}{3} r$ on our measured structure function. 


\section{Chapter 3 \\ Theory}

The old hady crowed triumphanth. "It's na ose, Mr. James-it's burtles all the war dowa!"

Discussion fullowing a lecture by William James

\subsection{The parton model}

The early experiments in the deep inelastic continunm shored that the aucleon behaves as if it contains pointlike constituents. That one could detect such structure meant that these constituents were interacting only weakly with each other. a fact not easily understood in the contest of the strong interaction which presumably bound them together. Understood or not. this bebavior is formalized as the parton model, the success of which is gratifying, if sometimes ba团ing.

In the parton model, the process of Fig. 2.1 is understood as the incoherent sum of diagrams sucb as Fig. 3.1. The nucleon is resolred into a swarm of partons. one of wich is responsible for the scattering. During the scattering the parton is assumed to be free. The scattering itself is simple. If the parion bas spin $\frac{1}{2}$, the virtual photon-parton vertex is calculated exactly as the lepton vertex was. Our ignorance about strong interactions is now relegated to the processes that determine the spectrum of partons in the nucleon and produce the splash of hadrons in the final state.

The parton model allows ns to rewrite the hadron tensor of Eq. 2.4. We consider the process in the frame where the nucleon momentum is large. We aeglect the transverse momentum of the partons and assign to each parton of type i a fraction of the nucleon's momentum $:$, from the noknow a distriburion $f_{1}\left(x_{1}\right) d z_{1}$. The hadron tensor is rewritten as

$$
\begin{aligned}
H^{-\mu \nu}=\frac{1}{2 \pi} \frac{E_{V}}{H_{Y}} \sum_{i} & \int f_{1}\left(x_{1}\right) d x_{1} \int \frac{d^{3} p_{2}^{\prime}}{(2 \pi)^{3}} \\
& \times\left[\frac{1}{2} \sum_{s p_{i n s}}<p_{1}\left|J^{\mu}(0)\right| p_{1}^{\prime}><p_{1}^{\prime}\left|J^{\nu}(0)\right| p_{1}>(2 \pi)^{4} s^{4}\left(p_{1}+q-p_{s}^{\prime}\right)\right] .
\end{aligned}
$$

The incoherent sum is now an integral over the initial distribution of partons, and the inclusive sum over fnal states is an integral over the final parton phase space. Aided by the momentum 


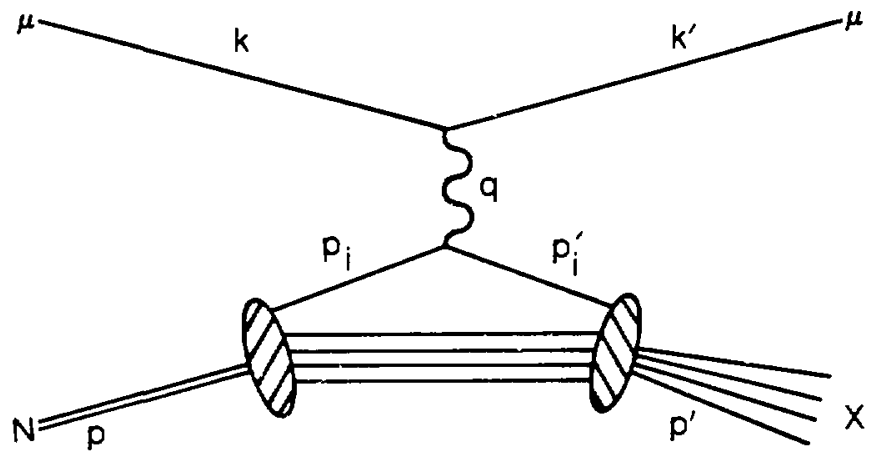

XBL 8310-709

Fis. 3.1. The parton model picture of deep inelastic scattering. 
conserving delta function, we perform the final state momentum integration. Because the leptonparton scatter is elastic and the partons are non-interacting, we are lef with the elastic scattering condition in the form

$$
\begin{aligned}
\delta\left(2 p_{i} \cdot q+q^{2}\right) & =\delta\left(2 \Sigma_{i} p \cdot q-Q^{2}\right) \\
& =\frac{1}{M_{N} \nu} \delta\left(x_{i}-\tau\right) .
\end{aligned}
$$

where $I=Q^{2} / 2 M_{N} \nu$ (see Eq. 2.2). We have neglected the masses and transverse momenta of the partons to allow the relation $p_{1}=x_{1} p$ to hold for all four momentum components. The measurable quantity $I$ is thus the fractional momentum of the struck parton.

We now calculate the matris elements. assuming the partons so be pointlike Dirac particles. Identifying the result term by term with the expression for $W^{\mu \nu}$ in Eq. 2.6 we find

$$
\begin{aligned}
& F_{1}(x) \equiv M_{N} W_{1}\left(Q^{2}, \nu\right)=\frac{1}{2} \sum_{i} f_{i}(x) e_{1}^{2}, \\
& F_{2}(z) \equiv \nu W_{2}\left(Q^{2}, \nu\right)=\sum_{i} x f_{l}(z) e_{1}^{2} .
\end{aligned}
$$

The structure functions $F_{i}$ are seen to be functions of $x$ only, with $e_{i}$ the charge of type- $i$ partons in units of the proton charge. This is the property known as "scaling."

Another property apparent from Eq. 3.3 is the relation $F_{2}(x)=2 x F_{1}(x)$. This is known as the Callen-Gross relation. ${ }^{20}$ Inspection of Eq. 2.11 shows that, in the Bjorken limit $\left(1^{2} / Q^{2} \rightarrow \infty\right.$ with $x$ fixed), the Callen-Gross relation implies $R=0$. This result can be understood in the frame where the parton backscatters from the virtual photon. Since the electromagnetic Dirac (spin $\frac{1}{2}$ ) current is helicity conserving, this scattering can take place only through the absorption of helicity $= \pm 1$ photons, hence $\sigma_{L}=0$ and $R=0$. For scalar partons. $\sigma_{T}=0$ and $R=\infty$. Allowing finite parton masses $m_{1}$ and transverse momenta modifies these conclusions $^{21}$ and leads to corrections of order $\left(m_{1}^{2}+p_{i \perp}^{2}\right) / Q^{2}$.

To summarize, in the parton model, a measurement of $F_{2}(x)$ is a determination of the moment um distribution of partons in the target nucleon - the aucleon 's "structure" in momentum space. A measurement of $R$ yields information on the spin of the partons themselves.

\subsection{Quantum chromodynamics}

At the beginning of the 1960 's, Gell-Mann and Ne'eman ${ }^{22}$ independently proposed SC(3) symmetry as the underlying structure of the cronded spectrum of strongly interacting particles. While the classification of hadrons into $S U(3)$ multiplets was completely successful. it was puzzling that , be fundamental 3 and $\overline{3}$ representations never appeared. Before long it was proposed independently by Gell-Mann ${ }^{11}$ and 2 weig ${ }^{12}$ that all observed hadrons could be considered to be composite particles, made up of combinations of "quarks" which transformed under the fundamental representations. The actual existence of quarks as phrsical (as opposed to mathematical) entitjes was a point of speculation. In fact, Gell-Maun proposed searches that "would belp to reassure us of the non-existence of real quarks"." The null results of such searches indicated that quarks were either very abstract or very tightly bound. In this environment, the experimentally-motivated parton model raised as many questions a it answered. If partons were quarks, then quarks had a rather robost (if restricted) physical existence inside nucleons and. furtbermore, scaling implied that they were quasi-free. 
(a)

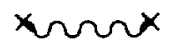

(b)

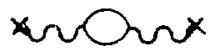

(c)

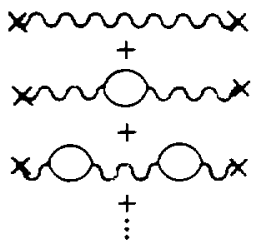

XBL $83 \cdot 0 \cdot 711$

Fig. 3.2. The QED photon propagator with higher order corrections.

With the existence of partons as both mrstery and clue, the development of a dynamical theory of tie strong interactions proceeded. Euclid's geometry and Tewton s mechanics were the explanatory standards of their day, and Kepler and Faraday felt compelled to try to cast their discoveries in terms of those successful structures. In high energy physics today, this role is played by quantum fleld theories, notably those with local gauge symmetry. The paradigm is quantum electrodynamirs, which through a perturbation expansion in the fine structure constant $\alpha \approx \frac{1}{31}$ provides a pcxerful and remarmbly accurate calculational tool. In creating a quantum fleld theory for the strong interactions of quarks one is in immediate diffculty. The coupling constant $a_{S}$ is not small. In fact, the apparently permanent binding of quarks into hadrons casts doubt on the use of quarks as the fundamental felus to begin with. However, just as the existence of real electrons with finite mass and charge was the key to bypassing the divergences of QED's perturbation theory, the existence of quasi-free partons in the nucleon offered hope that teyond the old-style strong interaction complexity, there was a usable field theory of puirks. In both cases, the restoration of flnite physical behavior to the fundamental felds is through renormalization.

In QED. the lowest-order contribution to the photon propagator (see Fig. 3.2a) gives a $1 / q^{2}$ dependence tnat is the Coulomb interaction. ${ }^{23}$ The second-order contribution of Fig. $3.2 b$ is infinite. Toget her, the expression for the propagator is ${ }^{i T}$

$$
\frac{-i g_{\mu \nu}}{q^{2}}\left[1-\frac{a}{3 \pi} \ln \frac{M^{2}}{m^{2}}+\frac{2 \alpha}{\pi} \int_{0}^{i} d z z(1-z) \ln \left(1-\frac{q^{2} z(1-z)}{m^{2}-i t}\right)\right] .
$$

where $m$ is the electron mass and $\mathcal{H}$ is a large mass inserted as a cutot. For $q^{2} \rightarrow 0$ we restore the Coulomb potential by renormalizing the coupling constant (or, equivalently, the electron charge) to absorb the formally inflate factor

$$
1-\frac{\alpha}{3 \pi} \ln \frac{M^{2}}{m^{2}}
$$


(a)

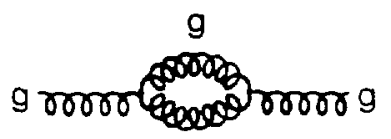

$\mathrm{g}$ (b)

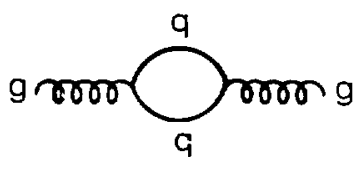

XBL 8310.710

Fif. 3.3. Corrections to the QCD gluon propagaior.

This procedure is defined to yield the obserfed coupling const3nt

$$
\alpha=\alpha_{B}\left(1-\frac{\alpha_{B}}{3 \pi} \ln \frac{M^{2}}{m^{2}}\right) \approx \frac{1}{13 i} .
$$

where $a_{B}$ is the unmeasurable "bare" coupling constant. The remainder of Eq. 3.4 is finite, $q^{2}$ dependent. and observable. ${ }^{24}$ In the imit of small $q^{2}$ it gives a small coctribution to the Lamb shift. If, instead, we look at the limit $Q^{2} \equiv-q^{2}$ large, we find a correction to the propagator of

$$
\frac{\alpha^{2}}{3 \pi} \ln \frac{Q^{2}}{m^{2}}
$$

We can choose to redefine $\alpha$ to include this correction as well. We restore Coulomb's law, but at the expense of introducing a $Q^{2}$ dependent or "running" coupling constant

$$
\alpha\left(Q^{2}\right)=\alpha\left(I+\frac{a}{3 \pi} \ln \frac{Q^{2}}{m^{2}}\right) .
$$

We could, in a similas fashion, include higher order contributions from a series of loops (Fig. $3.2 c$ ). Summing this series gives

$$
\alpha\left(Q^{2}\right)=\frac{\alpha}{1-(\alpha / 3 \pi)\left(\ln Q^{2} / m^{2}\right)}
$$

The runnidg coupling constant is seen to increase slowly with $Q^{2}$. This is interpreted as the penetration of the sbielding due to racuum polarization as one probes shorter discances, revealing morc oi the bare charge.

The theors of strong interactions was developed in analogy to QED. with an internal quamum number called "color" taking the part of the electric cbarge. The resulting theory" is thus known as quantum chromodynamics (QCD). The primary difference between QCD and QED is that, while photons are neutral. the "gluons" of QCD carry color and thus couple directly to one another. This allows diagrams such as Fig. 3.3a to occur in addition to the QED-like diagram of Fig. 3.3b. lncluding the new QCD diagrams in the calculation of the running coupling constant 
(a) $\operatorname{ror}\left(\frac{3}{2}\right) r$

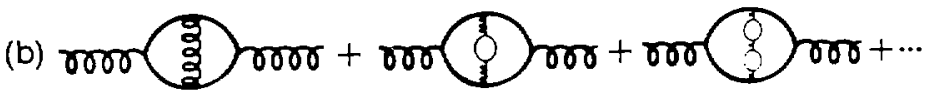

XBL 8310.712

Fig. 3.4. (a) A non-leading-log diagram. (b) The effect of using the renormalized coupling constant in (a).

for the strong interaction gives

$$
\alpha_{S}\left(Q^{2}\right)=\frac{\sigma_{S}\left(\mu^{2}\right)}{1+\left[\left(33-2 V_{f}\right) / 12 \pi\right] \alpha_{S}\left(\mu^{2}\right) \ln \left(Q^{2} / \mu^{2}\right)} .
$$

where $\mu^{2}$ is an arbitrary renormalization point and $V_{f}$ is the number of quark favors (up, down. strange...). This expression is distinguished frow the QED case by two features: the QCD coupling constant runs much faster (due to the presence of $\alpha_{s}$ in the denominator) and it runs in the opposite direction, getting weaker as $Q^{2}$ increases, for $N_{f}<17$. This latter property, known as "asymptotic freedo $m^{\text {" }}{ }^{25}$ is the key to the success of the parton model, and hence to the success of $\mathrm{QCD}$, as will be discussed furt her below. The restriction on the number of flavors arises because quark loop contributions behave like the electron loop terms of QED and run $\alpha_{S}$ up. They must be dominated by the remaining $Q C D$ terms to give asymptotic freedom. The expression Eq. 3.10 for $\alpha_{S}$ is an approximation based on ignoring diagrams such as Fig. 3.4a. In estimating its contribution : can use Eq. 3.10, or equivalently the renormalized gluon propagator, which automatically replaces Fig. 3.4a with the series of Fig. 3.4b. The asymptotically free running coupling constant increases the level of convergence and :he resulting contribution is of the order $\ln \left(\ln \left(Q^{2} / \mu^{2}\right)\right)$. The deglect of these terms compared with the $\left(\ln \left(Q^{2} / \mu^{2}\right)\right)^{n}$ behavior of the terms in Fig. 3.3 is known as the "leading logarithm" approzimation. We can remore the reference to the arbitrary renormalization point $\mu$ in Eq. 3.10 by introductag the parameter $A$ such that

$$
\alpha g\left(Q^{2}\right)=\frac{12 \pi}{\left(33-2 V_{f}\right) \ln \left(Q^{2} / L^{2}\right.}
$$

While $\mu$ xas arbitrary and unmeasuratle, $\boldsymbol{A}$ is defined by Eq. 3.11 and is measurable. Other definitions of $A$ differ from this one in the next order of $a_{s} .^{28}$ heeping the nest-to-leading logarithm gives such a correction. For this reason. $A$ as defined in Eq. 3.11 is sometimes called ito for "lowest order."

Asymptotic freedom allows the understanding of the quasi-free appearance of partons in nurleons. As in QED, the senormalized coupling is indeed small. Tisis permits meaningful 
perturbation series calculations for strong interactions at large $Q^{2}$. At the lor $Q^{2}$ end. it is hoped, but has not been proven, that the increasing $\alpha_{S}$ is a sign that the QCD interaction between quarks is confining. meaning that free quarks cannot be separated from their parent hadrons. Even without such proof, it is clear from Eq. 3.11 that perturbative QCD cannot work at low $Q^{2}$. For this reason, perturbative $Q C D$ cannot give predictions for such static properties of hadrons as the $z$ distribution of partons. With low $Q^{2}$ information as input, bowever, $Q C D$ can calculate the $Q^{2}$ dependence of such quantities. (Recall that the parton model predicts scaling. that is, no $Q^{2}$ dependence at fixed $I$.)

The most direct prediction of $Q C D^{13}$ is not of the $Q^{2}$ evolution of parton distributions. but rather of moments of those distributions, defined as

$$
M_{n}\left(Q^{2}\right)=\int_{0}^{1} d x x^{n-1} f_{i}\left(I, Q^{2}\right) .
$$

Given the moment at a reference $Q^{2}=Q_{0}^{2}, Q C D$ predicts the evolution

$$
M_{n}(t)=M_{n}(t=0)\left[\frac{\alpha_{S}(t=0)}{\alpha_{S}(t)}\right]^{d_{*}},
$$

with $t \equiv \ln \left(Q^{2} / Q_{0}^{2}\right)$ and the exponents $d_{n}$ calculable in perturbative $Q C D$. After calculating the moments to suffieiently high $n$, the set can be inverted to give results for the distributions themselves. When this is done, it is found that QCD predicts scaling violations which are logaritbmic in $Q^{2}$.

Fortunately, Altarelli and Parisi ${ }^{27}$ hare given a more direct interpretation of QCD: predictions, an interpretation that is a natural extension of the parton model. Figure 3.5a shows the parton model diagram for deep inelastic scattering vid a virtual photon. Allowing the partons to interact generates processes such as those in Figure $3.5 \mathrm{~b}-\mathrm{d}$. These processes rob from the momentum fraction I carried by the struck parton. We therefore expect that these processes will reduce the structure function at large $I$ and increase it at small $I$. Increasing $Q^{2}$ resolves finer and iner structure of this type, but asymptotic freedom slows the erolution. learing soth (logarithmic) scaling violations. The method of Altarelli and Psrisi accounts for the processes in Fig. 3.5b by writing a set of transport-ilke differential equations or the quark distribution functions:

$$
\begin{aligned}
& \frac{d \sum_{i=1}^{2 N_{t}} f_{s}(x, t)}{d t}=\frac{\alpha_{S}(t)}{2 \pi} \int_{I}^{t} \frac{d y}{y}\left[\sum_{i=1}^{2 N_{f}} f_{1}(y, t) P_{\mathrm{qq}}\left(\frac{x}{y}\right)+2 N_{j} G(y, t) P_{\mathrm{qG}}\left(\frac{z}{y}\right)\right] . \\
& \frac{d G(x, t)}{d t}=\frac{\alpha_{S}(t)}{2 \pi} \int_{I}^{1} \frac{d y}{y}\left[\sum_{1=1}^{2 N_{t}} f_{t}(y, t) P_{G Q}\left(\frac{x}{y}\right)+G(y, t) P_{G G}\left(\frac{x}{y}\right)\right] \text {. }
\end{aligned}
$$

The sum is over quark +antiquark flarors. and couplings are assumed to be flavor independent . $G(x, f)$ is the distribution of gluons in the nucleon as a function of momentum fraction $x$. We have anticipated our use of a nuclear target with a nearly equal mix of protons and neutrons. This leads to a mixt ure of quarks which is nearly a flavor singlet. Since the gluon is also a flavor singlet. there is coupling between the quark and gluon evolutions. As the fawor independence of the couplings implies, the gluon does not couple to the difference between two quark types. For such a case ("non-singlet") the evolution is simpler:

$$
\frac{d}{d t}\left[f_{1}(x, t)-f_{J}(I, t)\right]=\frac{\alpha_{S}(t)}{2 \pi} \int_{x}^{1} \frac{d y}{y}\left[f_{1}(y, t)-f_{1}(y, t)\right] P_{\mathrm{qq}}\left(\frac{I}{y}\right) .
$$




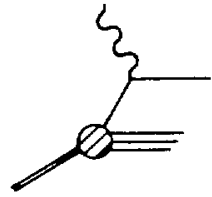

(a)

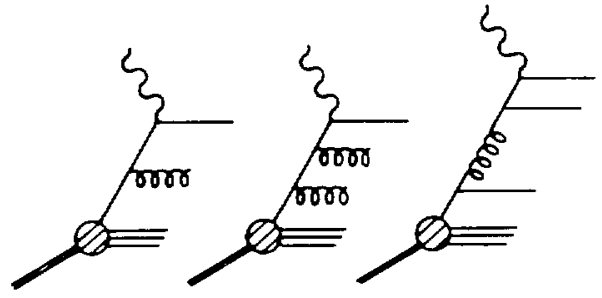

(b)

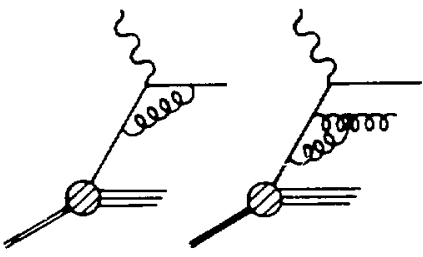

(c)

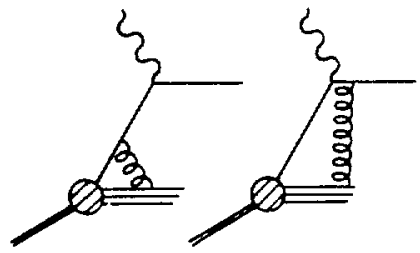

(d)

XBL $8310 \cdot 719$

Fig. 3.5. QCD modifications to the parton model diagram ! a) for deep inelastic scattering. (b) Logarithmic $Q^{2}$ evolution: (c) renormalization: (d) "higher twist". 

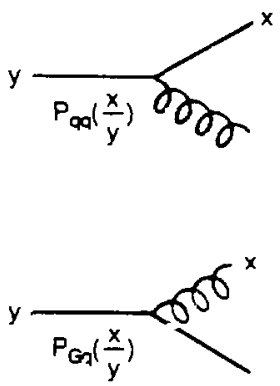
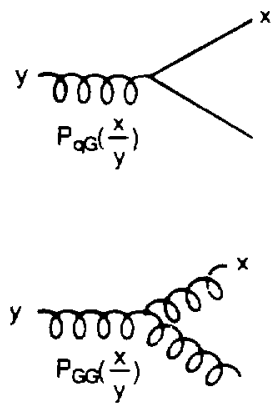

XBL $8310-718$

Fig. 3.6. QCD rertices governing the $Q^{2}$ evolution of the structure function.

The interpretation of Eqs. 3.14 is quite straightforard. The $P(z)$ 's re', rese at the probabilities of the processes in Fig 3.6, where a daughter parton (either quaris or gluon; is split from a parent parton, taking a fraction $z$ of its momentum. Thus partons of momentum fraction I (the left hand sides of Eq. 3.14 ) come from partons of larger moment um fraction $y$ with probability $P(x / y)$. This appreach is equivalent to the QCD calculation of moments: and the probabilities $P(z)$ can be calculated from the $d_{n}$ of Eq. 3.13 .

The diagrams of Fig. 3.5c and d still remain. Those such as that or Fig. 3.5c are take? into account automatically. They either cancel infrared divergences from the ewission of soft real gluons and are bandled by renormalization, or are contributions to the running coupling constant and thus are included in the leading $\log$ approsimation by using $\alpha_{S}\left(Q^{2}\right) .^{25}$ The diagrams of Fig. 3.5d are another story. Though not caiculable in detail, their general behavior is known. Since they involve interactions with more than one target quark, they resemble elastic scattering. where multiple gluon exchanges are required to keep the recoiling nucleon intact. This leads to a form factor-like suppression which goes as powers of $1 / Q^{2}$. At sufficiently large $Q^{2}$. the logarithmic beharior discussed above should dominate these terms, which bear the unfortunate name "higher twist".

To test the predictions of QCD. one can measure the structure function as a function of $Q^{2}$ for various fixed values of 3 . This $Q^{2}$ dependence or scaling violation can be compared to $Q C D$ by using the measured $I$ dependence at a fixed $Q^{2}=Q_{0}^{2}$ as the initial condition and integrating Eq. 3.14 to get predicted values of the structure function for all $Q^{2}$. For the singlet case. is is also necessary to bave $G\left(I . Q_{0}^{2}\right)$. the initial coadition for the gluon evolution. This distribution is not directly measurable. Neutrinns, however, are sensitive to the difference between quarks and antiquarks. With this essentiallr non-singlet information, various QCD and phenomenological parameters can be determined without complications from gluons. With these values fixed. tbe singlet structure functions can be used to extract of the gluon distribution. ${ }^{29}$ A hypothetically more direct but experimentally more difficult approach is to use the production of heavy flavors in the "photon-gluon fusion" diagram of Fig. 3.7 to tag interactions $\pi$ il h gluons. ${ }^{30}$ 


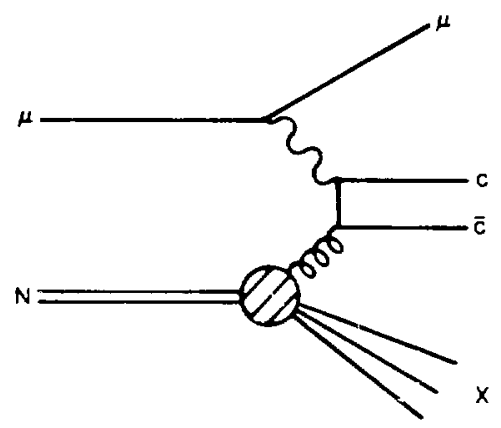

XBL 8310.713

Fig. 3.7. Photon-gluon fusisn. The production of a heavy quark pair can be used to lag the interaction with a gluon.

\subsection{Grand unified theories}

The realization that asymptotic freedom brought the strong interactions/at least at high $Q^{2}$ ) nader the hegemony of gauge theories brought a feeling of $x$ arm comport to high eisergy pbysics. All the forces of concern to high energy physicists (except the one that bolds thew to the planet, and the peculiar one that draws them to Batavia, Dlinois) were now described using the same basic tools. The weak and electromagnetic interactions had been uniffed and understood as vae gauge theory with a spontaneously broket symmetry. ${ }^{31}$ It was immedi :t ply hoped that the strong interactions could be added, making a "Grand linifled Theory" or GLT.

In a typical GLT (for a review, see Ref. 32.) quarks and leptons are placed in the same represent ation of a group $\left(S C^{\prime}(5)\right.$ for the simplest theor. $\left.{ }^{33}\right)$. Below some large energy scale charaterized by the mass $M_{X}$ of the bosons that mediate transitions among members of the representation, the symetry between the strong and electroweak interactions is broken. At 'ow energies symmet ry breaking is complete and we observe the $S\left[{ }^{-1}(3)_{\text {color }} \times S L^{-}(2) \times C^{-1}(1)\right.$ structure of the standard model. The mass $M_{Y}$ is determined by folloring the three runing coupling constants up to the energy where tbey become equal. I'sing o and us for this determination in one of the simpler models gives ${ }^{32}$

$$
M_{X} \approx 15 \times 10^{14} \mathrm{AIGeVI}
$$




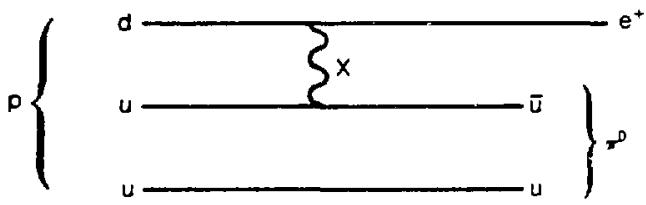

XBL 8310.714

Fig. 3.8. Proton decay urifiativ by $X$ boson exchange.

The symmeiry that allows transitions bet ween lepuns and quarks vic the exchange of $X$-bosons means that baryon nomier is not conserved and hence protons are no longer stable. Proton decay can proceed through the diagram of Fig. 3.8 und others. The predicted lifetime is

$$
\tau_{p} \sim \frac{1}{a_{G U T}} \frac{M_{X}^{4}}{M_{p}^{5}} \approx 5 \times 10^{32}\left[\Lambda\left(\mathrm{GeV}^{4}\right]^{4 r .}\right.
$$

with over an orde: of magnitude of theoretical uncertainty. The existence of this lepton-quark symmetry and its breaking has a number of other uses. It explains the apparently exact equality of the magnitude of the charges of the electron and proton. Baryon number violation is necessary. but not sufficient, to explain in a dynamical way the nbserved matter-antimatter asy mmetry in the universe. ${ }^{34}$ Some speculative theories use the "super-saturated" state of the universe in the false vacuum prior to the spontanevis breakdown of symmetry to give a cosmological constant. that generates the entire observable universe out of virtually nothing. ${ }^{35}$

Although GCT's have had some theoretical successes, the prediction of proton dec' $y$ is one of the fer that may he reriflable. Early determinations of 1 indicated values on the order of several bundred $\mathrm{MeV}$. $\mathrm{Th}$ : resulting proton lifetimes were above, but close 10 , existing experimental lower limits of $\approx 10^{29}$ years. ${ }^{36}$ A new generation of proton decay experiments designed to probe the GLT regime has recently begun running. Early results ${ }^{3 z}$ Ior the decay mode $p-e^{+} \pi^{0}$ yield limits in conflict. with the simplest GLT models. The fourt h-power dependence of ip on 1 malies a reliable determination of $A$ essentjal to the confrontation of theors with experiment. 


\subsection{Theoretical summary and experimental program}

In the brief time since this experiment was proposed, the amphasis of theoretical interest has changed a great deal. Through all these shifts, the importance of input from deep inelastic lepton nucleon scattering has persisted. The original measurements of this type were concerned with determining the geometrical structure of the target. Today the diflerential cross section Eq. 2.12 is dissected to give information on a variety of topics. At fixed $Q^{2}, F_{2}(J)$ gires the longit udinal moment um distribution of the nucleon's charged constituents. $R$, determined using $F_{2}(E)$ at fled $Q^{2}$ and $a$ (see Sec. 10.6), gires the spin of the constituents and can be affected by their transverse momenta. At fixed $x$, the $Q^{2}$ dependence of $F_{2}$ probes the interactions of quarks and gluons and determines the running coupling constant of QCD. Grand unification turns a measurement of $a_{S}\left(Q^{2}\right)$ into a prediction or the proton lifetime.

The experimental program is clet: test this theoretical struct ure by making a precision measurement of the differential cross section of deep inelastic scattering over as broad a kinematic range as possible. This is especially important for $Q^{2}$, where the rariation of interest is expected to be only logarithmic (on top of a $1 / Q^{4}$ fallofi) and the low $Q^{2}$ region may be obscured by nonperturbative effects. The experimental considerations for such a measurement will be discussed in the next chapter. 


\section{Chapter 4 \\ The Multimuon Spectrometer}

The mud elephant, wading through the sea, kares do tracks.

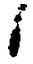

The Fugs, c. 1970

\subsection{Design constraints}

Our goal is to measure the nocleon structure function $F_{2}\left(x, Q^{2}\right)$ over as large a range of $Q^{2}$ as possible, without being forced to include very low $Q^{2}$. The kinematic limit, $Q^{2}<2 M_{N} E$ (see Eq. 2.2), requires us to have a high energy lepton beam. The desire to reach $Q^{2}>100 \mathrm{GeV}^{2} / c^{2}$ rules out existing electron beams, so we use the high energy inuon beam at Fermilab. The virtual photon propagator suppresses the large $Q^{2}$ cross section like $1 / Q^{4}$. To reach high $Q^{2}$ we must therefore have high luminosity. We achieve high luminosity by using a high intensity beam incident on a long, heavy target. To maintain high acceptance throughout the long target, the target and the spectrom:ter used to analyze the final state are integrated into one pachage. The high magnetic flelds required for momentum analysis above $100 \mathrm{GeV} / c$ and the large magnetized volume require an iron magnet.

Along with deep inelastic scattering. the experiment is designed to observe multimuon flnal states: $\mu N \rightarrow \mu \mu X, \mu \mu \mu \mathrm{N}$, etc. An example is shown in Fig. 3.i, where the heary quark state decays to one or more muons. ${ }^{30,38}$ This imposes three constraints: suppression of low mass electromagnetically produced muon pairs; observation at the lower $Q^{2}$ s typical of multimuon production (a few $\mathrm{GeV}^{+2} / \mathrm{c}^{2}$ ) and thus at smallet scattering angles: and suppression of secondary muons from pion and kaon decay in hadronic showers. To meet the first requirement. we use a Cartesian geometry with a uniform vertical magnetic fleld for momentum aralysis. Mivon trajectories bend (and muon pairs separate) in the horizontal plane. For pair mass discrimination we demand a minimum vertical opening angle, which is unaflected by the magnetic separation. When the large $(\approx 20 \mathrm{~cm})$ size of the beam is folded in, the second constraint requires that the spectrometer be active in the beam region and therefore that the magnetic fleld extend uniformly to the center of the beam. This dictates that the target material be the same iron used in the magnet. This target must be densely packed along the beam direction to suppress $\pi$ and $K$ decay.

The result is the Multimuon Spectrometer (MMS) illustrated in Fig. 4.1. The MMS is a horizontal stack of 91 4inch thick steel plates, each eight feet on a side. The stack is 


\section{Multi-Muon Spectrometer Berkeley-Fermilab-Princeton}

$S_{1.12}$ in modules $4,6,8 \ldots 18, P C+D C$ in $1.18,5 C$ in 1.15

(b) Section

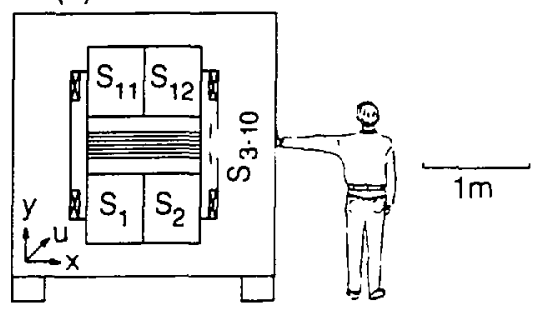

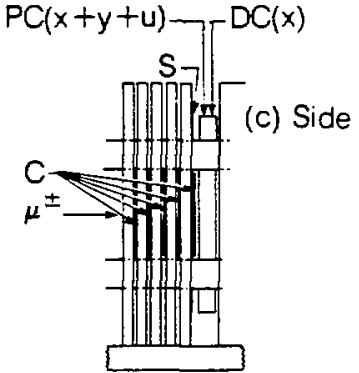

(a) Top

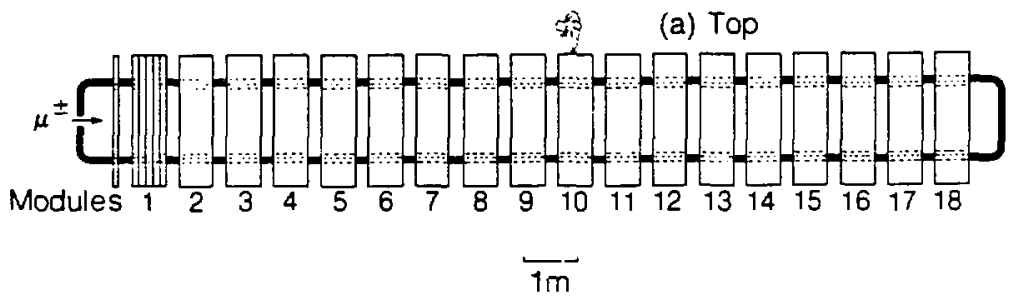

XBL 8310.733

Fig. 4.1. The Mutiman Spectrometer (MMS). (a) Top vier, showing the arrangement of iron plates into modules and the magnet coils running the length of the stack. (b) Section, looking into the beam, showing the coils in their slots, our coordinate system, and a trigger scintillator hodoscope. Paddles $S_{1,2,11,12}$ are used to signal a scattered muon, while stares $S_{3-10}$ serve as a beam reto. (c) Side riew of one module - detailed in Fig. 4.4. 
magnetized by coils running the length of the stack through slots cut in the plates. The iron serves as target. analyzing magnet, hadron absorber/muon identifier, and badron calorimetry medium. The spectrometer is instrumented with multiwire proportional and drift chambers for recording muon trajectories and with plastic scintillation counters for triggering and calorimetry The rarious components of the MMS are described in the remaining sections of this chapter.

It should be noted that the MMS solution to these constraints represents a compromise and that other solutions have been designed and operated. The effectively solid-iron environment in which we work is a messy one, complicating the reconstruction of tracks and limiting our momentum resolution. An alternative design sacrifles luminosity for better resolution and a simpler target material by using a hydrogen or deuterium target folloned by an air gap magnet. with muon identiflcation postponed until after the magnet. Examples are the experiments done in the Chicago Cyclotron Magnet at Fermilab ${ }^{18}$ and by the European Muon Collaboration at CERS. ${ }^{30}$ For the reasons discussed above, these experiments are not suitable for the study of multimuon production. Another alternative is to use a neutrino beam. This probes somewhat different physics and is a complement to the muon program.

\subsection{Operational summary}

- Protons from the Fermilab stachrotron incident on a target produce pions whicb decay, giving a beam of muons. (Fig. 4.2)

- The last two magnets in the muon beam line are instrumented to record the beam track for momentum analysis. Beam muons arc rejected if accompanied by a second inuon. (Fig. 4.3)

- The beam muon enters the MMS. Three in a million scatter in the iron by enough to enter trigger counters above or below the beam. generating a signal to record an event. (The multimuon triggers operate in parallel.)

- The bending trajectories of the beam and scattered muons are recorded in proportional and drift chambers located at Qve plate intervals throughout the MMS. (Fig. 4.4)

- The pulse heights in plastic scintillators in the beam region after every plate are recorded to measure calorimetrically the energy of the flnal state hadronic shower.

- Our coordinate system is deflned with $z$ along the beam axis. $y$ up. and $z$ horizontal.

\subsection{The muon beam}

The production of the Fermilab maon beam begins with the sontinuous extraction of the $400 \mathrm{GeV}$ proton beam during the $1.25 \mathrm{sec}$ "flattop" of the accelerator. Typically $1.5 .2 \times 10^{13}$ protons were accelerated per cycle, with over half delivered to a $30 \mathrm{im}$ aluminum target for the $\mathrm{Nl}$ muon line. The pions and kaons produced in the target were focussed into a $400 \mathrm{~m}$ emcuated decay pipe (see Fig. 4.2) where the pions and kans decayed to muons. The beam was sign- and momentum- selected in dipoles D1 and D2 and focussed into the aperture of D3 which contained 60 feet of polyethelene $\left(\mathrm{CH}_{2}\right)$ absorber to remove the remaining hadrons. The heam was then bent and focussed into the muon lab. The acceleration cycle was repeated at intervals of approsimately $10 \mathrm{sec}$. The resulting beam yielded approximately $1\left(\frac{1}{3}\right) \mu^{+}\left(\mu^{-}\right)$per $10^{-}$protons on target with $8 \pi / \mu$ ratio of less than $10^{-i}$ and 3 momentum acceptance of $\pm 2.5 \% \mathrm{c} .{ }^{10}$ 


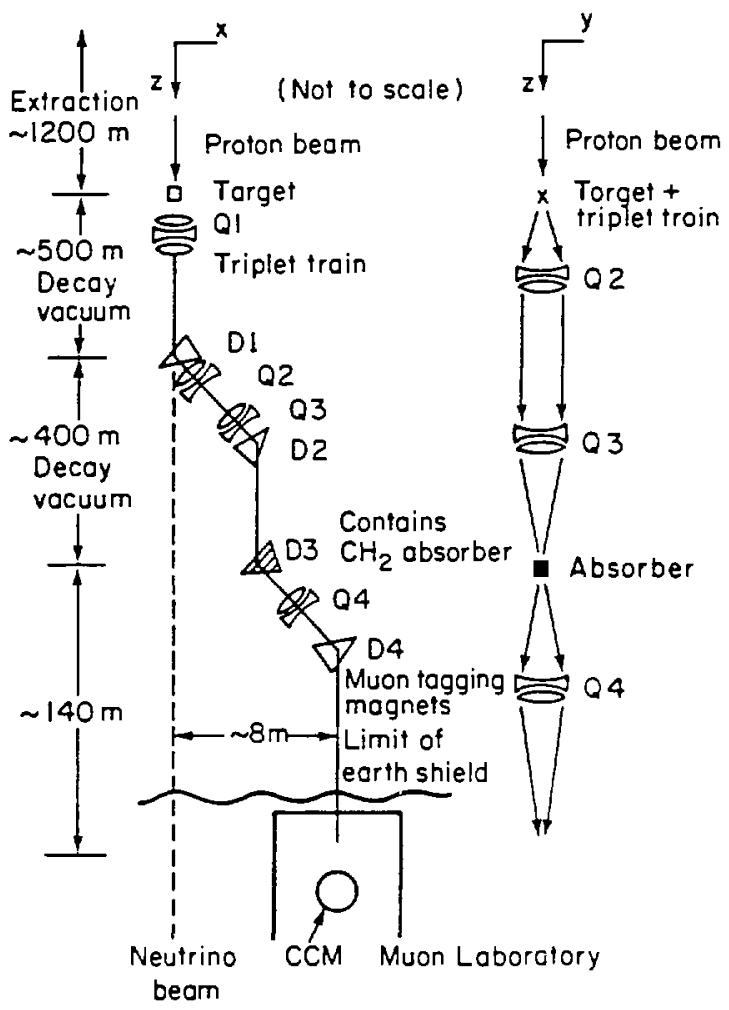

XEL 8010-2137

Fig. 4.2. The Fermilab muon beam. 


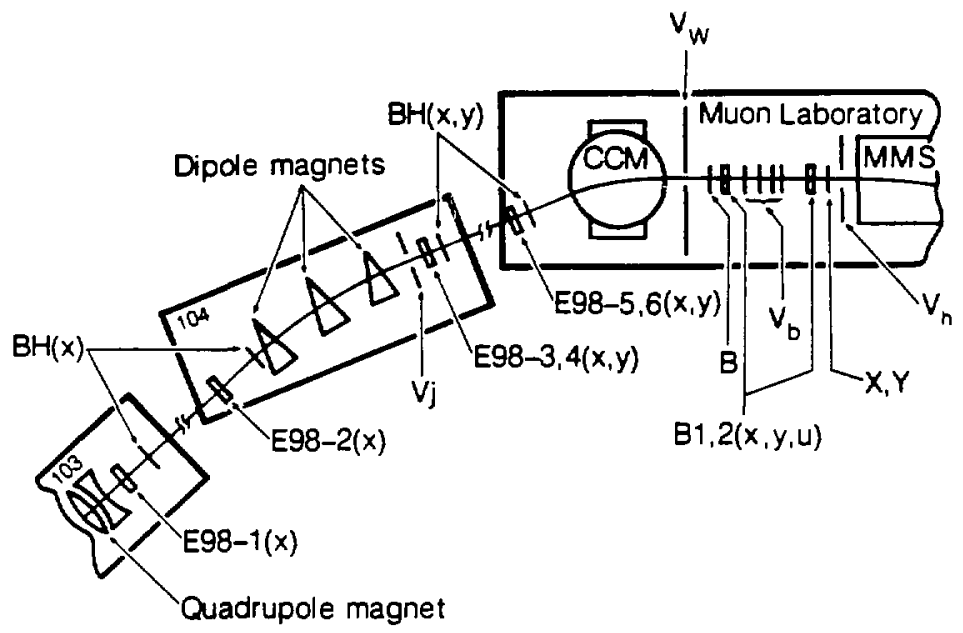

$\begin{array}{ll}\text { E98-n, B1, B2 } & \text { Multiwire proportional chambers } \\ B H, X, Y & \text { Beam scintillator hodoscope } \\ V_{j} & \text { "Jaw" veto counter } \\ V_{w} & \text { Large veto wall } \\ B & \text { Beam defining scintillator } \\ V_{h} & \text { Halo veto hodoscope } \\ V_{b} & \text { Bucket occupancy veto counter } \\ \text { MMS } & \text { Multimuon Spectrometer } \\ \text { CCM } & \text { Chicago Cyclotron Magnet }\end{array}$

XBL 8310.721

Fig. 1.3. The beam monitoring system used to define a valid beam muon and determine its momentum. Not to scale. 
Beam instrumentation is shorn in Fig. 4.3. Experiments using the Chicago Cyclotron Magnet (CCM) ran simultaneously with the MMS, which was positioned along the CCM-deflected beam line. The last $\mathrm{N1}$ dipole and the $\mathrm{CCM}$ were instrumented with multiwire proportional chambers (MWPC) and scintillator bodoscopes, (E98-1...6, BH) giving four horizontal $(\tau)$ and two vertical $(y)$ measurements. These, in conjunction with two MUPC (B1 and B2) measuring $x, y$, and a diagona) ( $u$ ) coordinate located in front of the MIS, were used to determine the moment um of indiridual beam muons. ideally to better than $0.5 r_{c}\left(\sigma_{p} / p\right)$. In reality. the situation was complicated by material placed in the beam by the upstream experiment, including borax (to clean up the beam) and a lead-glass shower counter 20.5 radiation lengt hs thick. With enough INWPC information we could fit the Coulomb scattering angle in the lead-glass, but at the cost of redundancy in the momentum fit. Energ loss in material upstream of the MMS could be corrected for in an average sense onjy. Straggling was accounted for by including in in the simulation used for acceptance and resolution modeling (see Chap. 8).

A system of scintillator hodoscopes (see Fig. 4.3) was used to define the beam size and choose usable beam muons. The last focus of the beam was in the aperture of the last beam line dipole. At the front of the MLMS, the counter B and the hodoscopes $\mathrm{X}$ and $\mathrm{Y}$ deflned the beam to be $35.1 \mathrm{~cm}(x)$ by $22.9 \mathrm{~cm}(y)$. A usable muon was defined to be one unaccompanied by a second muon. either in or out of the beam. This was especially important to protect the multimuon triggers. The muon beam retained the $53 \mathrm{MHz}$ signature of the syocbrotron, leading to a time structure of 18.9 ns "If buckets." Signals from counter B were put into advanced or delayed anticoincidence to veto muons which bad another muon in either the preceding or following bucket. If another muon was in bucket \pm 2 or \pm 3 , the tiack was used but tagged. Tracks with accompanying muons in the same bucket were rejected on the basis of pulse height information from scintillators $B$ and $V_{b}$ and from hodoscope information from $X$ and $Y$. A $1.4 \times$ minimum ionizing signal from any three of the flre pulse heights or more than one count in eit her hodoscope plane retoed the track. Muons out of the defined beam area are known as "halo." In the Fermilab muon beam. total intensity outside our defined beam was roughly equal to the intensity in the beam. Beam muons with an in-time halo muon were rejected by three layers of veto scintillators. one at $V_{w}$ and two at $V_{h}$. The signal for a usable beam track was called BV (for "retoed beam"):

$$
\mathrm{BV}=\left(\mathrm{B} \cdot \sum \mathrm{X} \cdot \sum \mathrm{Y}\right) \cdot \overline{\mathrm{ADJ}} \cdot \overline{\mathrm{SAIIE}} \cdot \overline{\mathrm{V}}_{\mathrm{w}} \cdot \overline{\mathrm{V}}_{\mathrm{b}} .
$$

where $A D J$ is a signal from an acjacent bucket and SAME is a second muon in the same bucket. $\mathrm{BV}$ was required in coincidence with all physics triggers, and a prescaled number. typically $1 / 350000$, was recorded with no further trigger requirements for calibration and use as a unbiased input for the simulation programs. The counter B, designed to give large, well behared signals. was used further as the main timing element for the entire experiment. It was fanned out and used repeatedly in all triggers as a strobe to maintain the critical timing required by our comples trigger.

The intensity of the muon beam was $\approx 3 \times 10^{6}$ muons per pulsa or, equivalently. $a$ bucket occupancy probability of 0.07 . This meant that there was typically one muon in the mion lab at a time. The vetoes rejected from $\frac{1}{3}$ to $\frac{1}{2}$ of the muons. leaving a usable fus of $1.5-2 \times 10^{\circ}$ muons per pulse. For $\mu^{-}$running, the beam intensity was a factor of three lower, but the lower intensity meant less veto rejection. giring a usable fux of around $0.9 \times 10^{8} / \mathrm{pulse}$.

\subsection{Target and magnet}

The 91 steel plates of the Multimuon Spectrometer were rolled and cut at the Danly steel Mills of Chicago from AJSI 1018 steel with a carbon content of $(0.17 \pm 0.03) r_{c}$. The 
average thickness of the plates $\approx$ as $10.28 \mathrm{~cm}$. The plates were grouped into 18 modules of five. with $3 \mathrm{~cm}$ gaps separating the plates. The remaining plate, known as plate 0 . was placed at the front of the stack where the last plate of an otherwise nonexistent module 0 would have been. Following each module was a large gap for the insertion of an instrumentation package. This packige was designed to be as thin as possible to minimize total module length and thus maximize high $Q^{2}$ acceptance and average target density. The large gaps were typically $25.4 \mathrm{~cm}$ thick. This dimension was adjusted to give a module length of $88.90 \pm 0.04 \mathrm{~cm}$. A module with

instrumentation is shown in Fig. 4.4. The mass of the entire spectrometer was $4.3 \times 10^{5} \mathrm{~kg}$ or $4 i 5$ tons.

The target was restricted by trigger geometry to be the first 13 modules plus plate 0 . This gave $678.3 \mathrm{~cm}$ or $5.34 \mathrm{~kg} / \mathrm{cm}^{2}$ of iron. Including scintillator, chambers, and support structures gave a total target thickness of $5.61 \pm 0.01 \mathrm{~kg} / \mathrm{cm}^{2}$, where the error is combined uncertainty due to measurement error in the plate thickness and an estimate of uncatalogued material in tbe beam. This target thickness and our integrated flux of muons gave the experiment a sensitivity of nearly 1 event/femtobarn for unit acceptance.

The iron target was magnetized by 4000 amperes $\times 18$ turns of water-cooled copper running the entire length of the MVS through slots cut in the plates. The conflguration is shown in Fig. 4.5. The confguration and shape of the slots were designed to give an approximately uniform rertical magnetic fleld in the $107 \mathrm{~cm}$ wide by $179 \mathrm{~cm}$ high active area flling the region betreen the coils. The MMS was run for roughly equal durations in both fleld polarities to cancel any systematic left-right asymmetries in the apparatus. This cancellation was only approximate because the CCM was required by the alignment of the MMS and the location of downstream shielding always to bend the beam to the East. This meant that the low momentum tail of the beam was treated asymmetric ally by the two polarities of the MIIS. The benefits of field reversal were nonetheless substantial, especially because once the MMS is magnetized there can be no zero field running. The polarity was typically changed once a day.

The magnetic field was mapped and calibrated using three sets of measured information. Flux loops spanning 12 sections of each module and one large loop enclosing the entire magnet gare absolute measurements of the flus of $B$ in the iron. Hall probe and fip coil measurements between plates mapped the $z$ and $y$ components of $\mathbf{B}$ in the air gaps. Precision measurements of iron samples removed from the coil slots gave $B$ vs. $H .^{40}$ These measurements were turned iuto an absolutely calibrated field map for the MMS by consiraining the fleld to agree with both the measurements (used as boundary conditions) and the Maswell equations. ${ }^{41}$ The field integra] for an average module was $9.998 \times 10^{5}$ Gauss-cm for an average feld of $11.25 \mathrm{bG}(19.46 \mathrm{kG}$ in (he iron). The transverse momentum kick of the magnet was $0.300 \mathrm{GeV} / c$ per module. The field was mapped to $0.2^{\circ} \mathrm{c}$ and observed to be uniform to $3 \%$ in the active region of the spectrometer.

\subsection{Trigger}

Trigger hodoscopes of 12 scintillation counters were located after each even numbered module starting with module 4. There were eight trigger banks in all, separated by $102.8 \mathrm{~cm}$ of jron. Figure 4.6 shows a trigger bank, along $\$$ ith the rest of a large-gap instrumentation package. The central section of each trigger bank was a set of sis narrow staves, each $3.9 \mathrm{~cm}$ high and extending the width of the active area. These staves covered the beam region and were used primarily for multimuon triggering. Above and below the set of narrow staves was a single wide $(15.2 \mathrm{~cm})$ stave. These, along with the nurrow staves. made up the beam reto for the deep inelastic scattering trigger. The regions above and below the staves were each divided vertically into two "paddles" These paddles, each $60.5 \mathrm{~cm}$ high by $52.1 \mathrm{~cm}$ wide, signaled the 


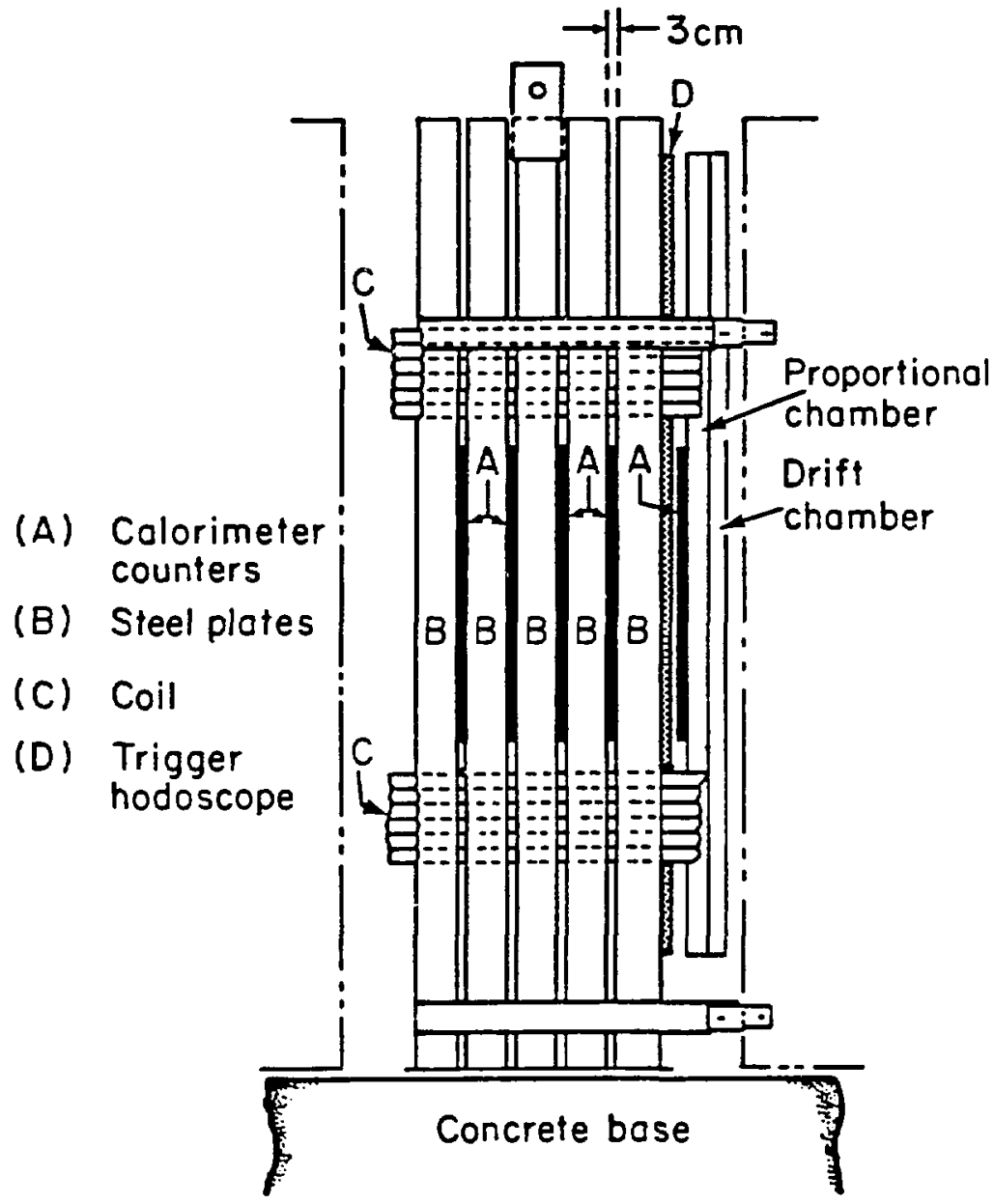

Fig. 4.4. An MLS module showing the location of the calorimeter scintillators and a large gap instrumentation parkage including a trigger hodoscope, a proportional chamber. and a drift chamber. 


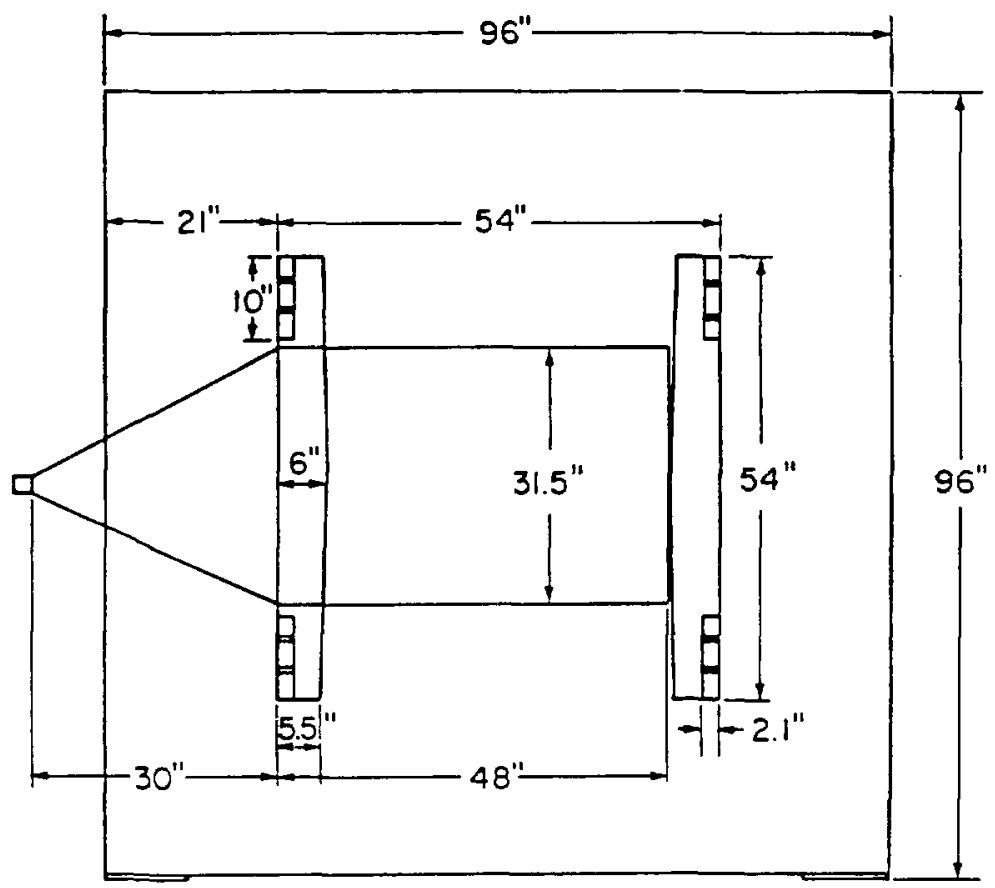

XBL $819 \cdot 7313$

Fig. 4.5. The larout of coil slots and coils in a single iron plate. Also shown is a calorimeter counter. 


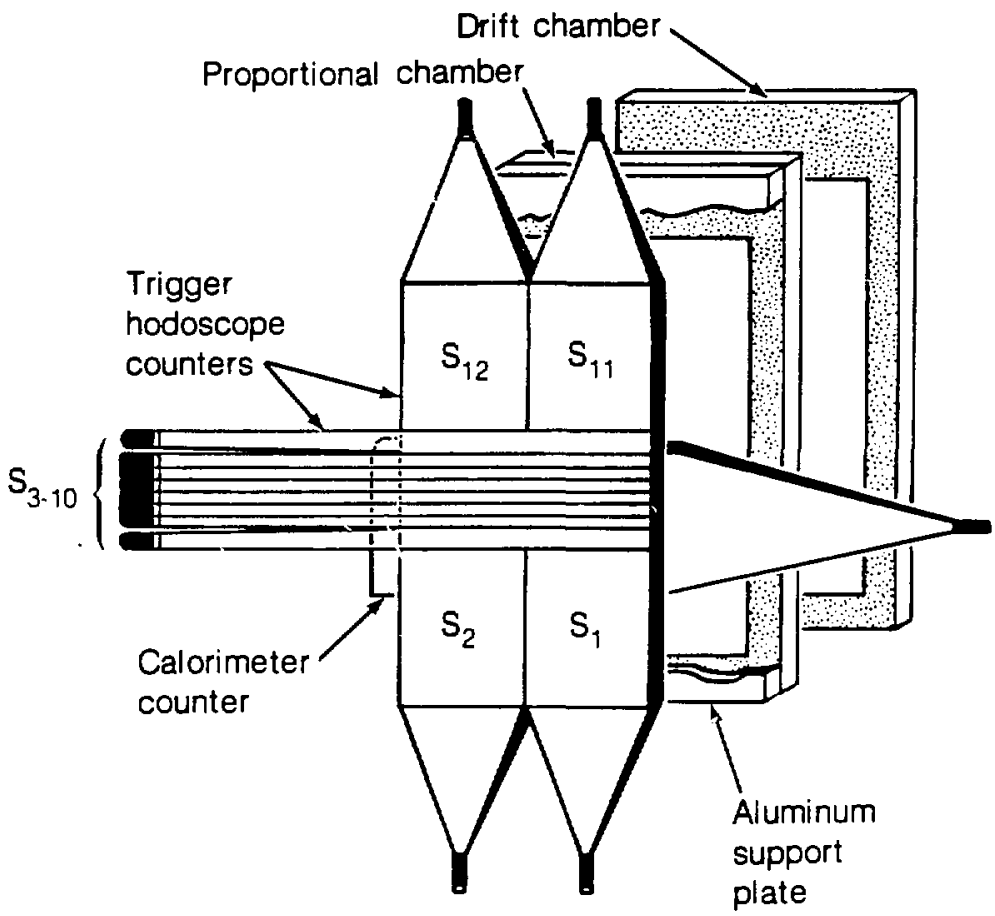

XBL $8310-720$

Fig. 4.6. A large gap instrumentation package. Trigger hodoscopes were located in eren numbered gaps only, starting with module 4 . 
presence of a scattered muon. The deep inelastic trigger required a signal from any paddle and no signal in the beam veto (staves) in each of three or more consecutive trigger banks. There \#3s no requirement made on badronic energy deposition. The eight trigger banks made up six overlapping subtriggers of three banks each. These operated independently and a signal from any of them in coincidence with a BV signal from the beam logic (Eq. 4.1) created a full trigger signal which initiat the readout of the spectrometer information and the recording of an erent. Along with chamber and calorimeter information. the logical status of each trigger and beam counter and each subtrigger were recorded. The trigger rate for the deep inelastic trigger was $3 \times 10^{-6}$ per usable beam muon.

The xide staves between the paddles and the beam region imposed a minimum vertical scattering angle requirement of $12 \mathrm{mr}$. This minimum could only be reached by a scatter in module I triggering the last subtrigger. Moving the interaction rertex downstream increased the minimum accepted scattering angle. The acceptance of tac MMS in $Q^{2}, I$, and $E^{\prime}$, areraged orer the length of the spectrometer, is show $\mathrm{a}$ for beam energies of 93 and $215 \mathrm{GeV}$ in Fig. 4.7. Since the cross section goes to infinity and the acceptance goes to zero as $Q^{2} \rightarrow 0$ the absolute value of the acceptance for a kinematic region that includes very low $Q^{2}$ is arbitrary. The plots assume $:>0.015$. or $Q^{2}>2.5,5.9 \mathrm{GeV}^{2} / \mathrm{c}^{2}$ for tne two beam energies. Figure 4.8 shows the acceptance in ihe $Q^{2}-I$ plane. Finally, Fig. 4.9 shows the acceptance as a function of rertex : location for several ranges of $Q^{2}$. All of these results are from the Monte Carlo simulation discussed in Chap. 8.

The deep inelastic trigger was relatively free from background. The primary source of non-physics background, that is, triggers not involying an actual scattered muon. Was a stopping or decaying beam muon (to give a $\mathrm{BV}$ signal. yet avoid the beam-vetoing staves) in coincidence with a halo muon, lobbed over or under the halo veto and entering the MMS from the top or bnttoru. directly into the paddles. The chief source of this component of the halo mas believed 10 be ot herwise barmless halo muons deflected back toward the beam by the return yoke of the Chicago Cyclotron Magnet. Approximately $1.6 \%$ of the triggers were from this source. They Here easily eliminated in analysis by the failure of the beam and "scattered" tracks to meet at a consistent rertex.

During the data analysis. we discovered that some of the paddle counters were quite ineticient. Worse, the ineficiency was spatially non-uniform. Since the $x$ and $y$ positions of a track at the back of the MTS translate quite directly into $E^{\prime}$ and $\theta$ due to the Cartesian geometry of the spectrometer. this position dependent efficiency was a disaster. Fortuntely. the redundant nature of the trigg r with its orerlapping subtriggers. allowed us to use the dat a to completely map the trigger eff ciency. Tbis crucial saga is related in sec. 7.5 . The stave counters were measured to be $>99^{\circ} c$ eflcient.

\subsection{Proportional and drift chambers}

Yuon trajectories in the MMS nere determined from positions measured after every module in packages containing three multiwire proportional chamber (PC) planes and one drift chamber (DC) piane (see Figs. 4.1,4, and 6). There were 19 such packages. inclading one following plate 0 , and the entire system contained over $14 \mathrm{nnn}$ cbannels. The chambers covered an area $106.7 \mathrm{~cm}$ wide by $178.9 \mathrm{~cm}$ higb. slightly larger tnis that covered by the trigger hodoscopes.

Each PC was built on a $1 / 2$ inch aluminum jig plate bept at ground potential, which formed the entry window. Continuing in the beam direction, the jig plate was followed by a catbode plane of wires oriented at 60 degrees from rertical, an anode plane of vertical wires. a second cat hode plane of horizontal wires, and a 1/16 inch aluminum xindor at ground. The 


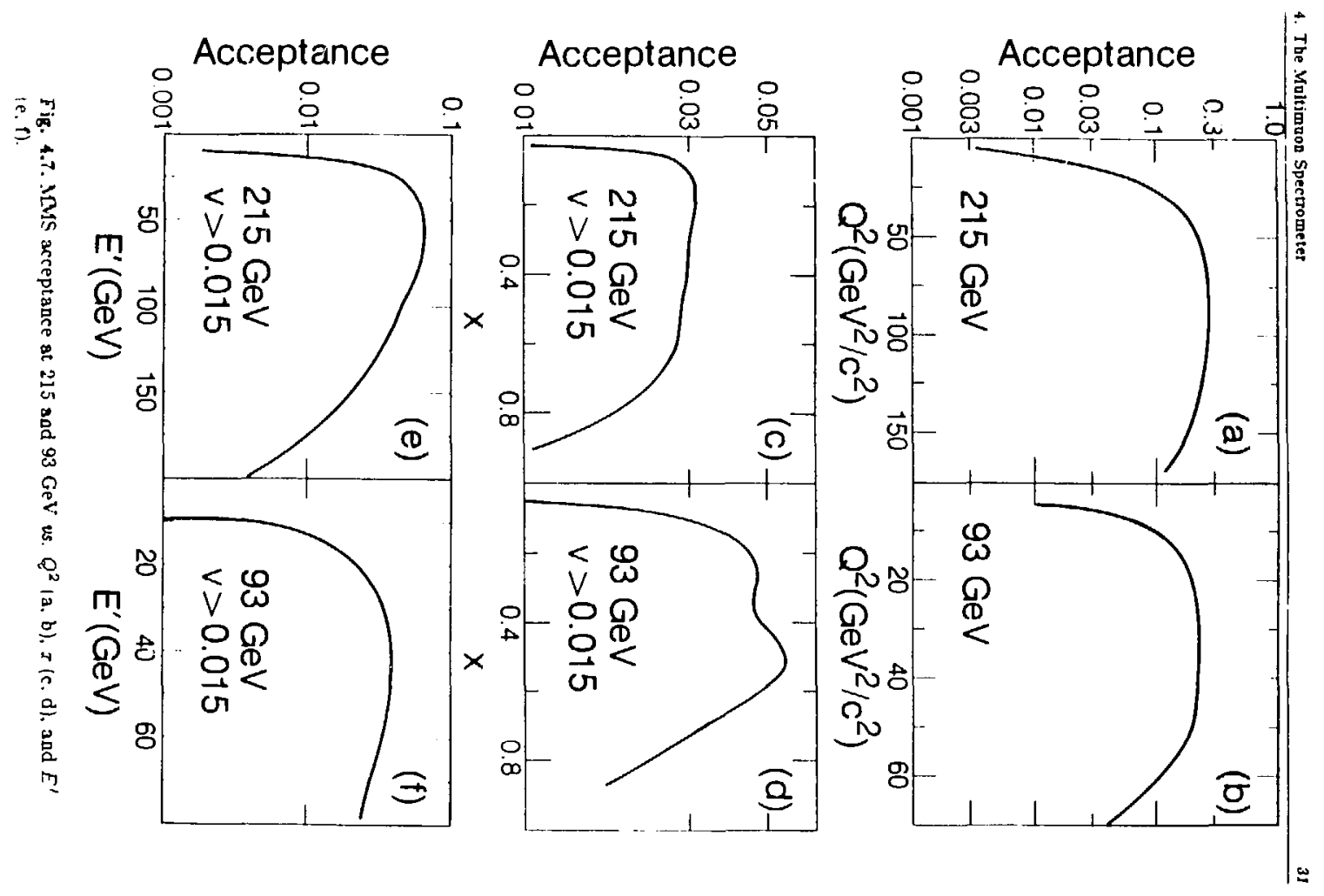




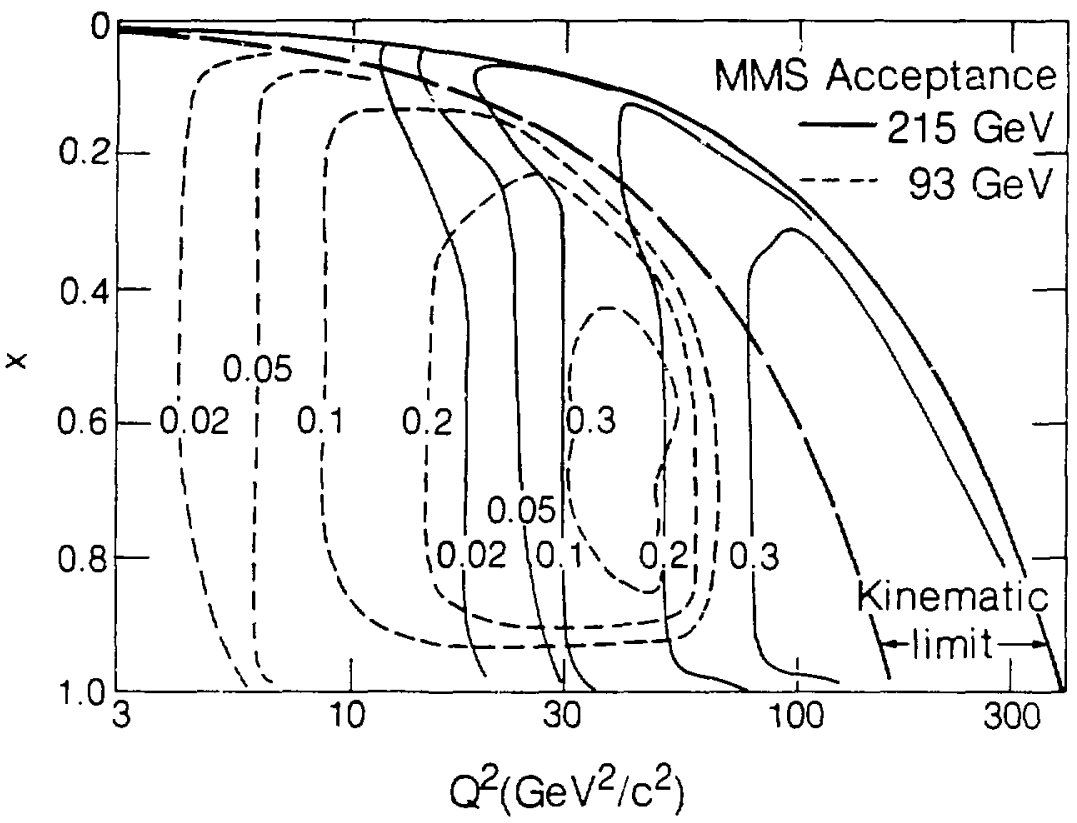

XBL $8310 \cdot 726$

Fig. 4.8. Contours of constant MUIS acceptance us. $Q^{2}$ and $I$. The $x$ axis origin is at we top to facilitate comparison with the $F_{2}\left(x, Q^{2}\right)$ plots of Chap. 10. Solid (dashed) contours are (cr a beam energy of $215(9.3) \mathrm{Ger}$. 


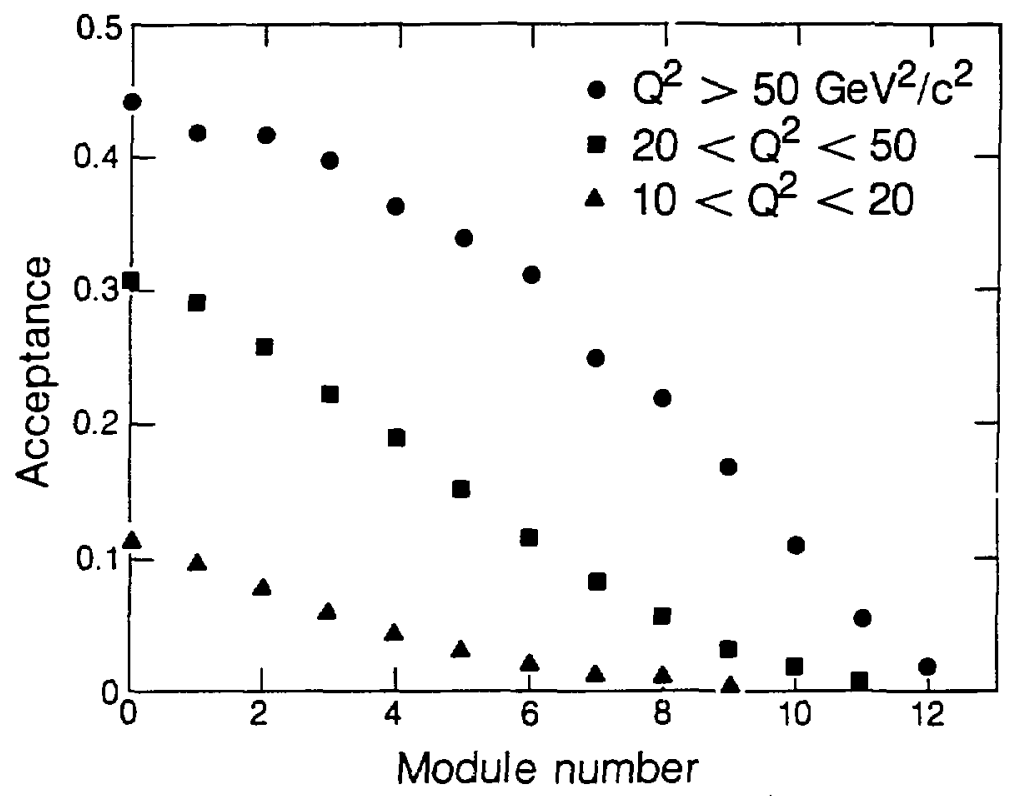

XEL B311.766

Fis. 4.9. MMS acceptance at $215 \mathrm{GeV}$ vs. vertex location. 
anode (sense) plane, measuring the $I$ coordinate, was formed of 336 wires with 0.125 in spacing. Each wire was de conpled to an amplifier/discriminator. The boundary between modules was defined to be at the sense plane. and the sense plane in PC 0 was defined as the origin in $z$. The signals induced on the cathode planes were read out to measure $y$ and a diagonal $u$ coordinate. The cathode wires were 0.050 inches apart and ganged together in groups of four. The broad induced signals were discriminated only after passing through a center-finding circuit (Fig. 1.10 ) with an output that was the second difference of the input. (Returning to our vision analog: this is the same circuit used for pre-processing of risual signais in the retina (Fig. $4.10 \mathrm{c}$ ). ${ }^{42}$ It is apparent!y used for edge-enhancement and is generally unnoticable except in the visual illusion known as Mach bands. ${ }^{43}$ ) Although there are only half as many output channels as input cbannels, resolution equivalent to the input channel spacing is restored by the design, which gives signals on either one or two output channels, depending on which of the two input channels is hit (compare Figs. $4.10 \mathrm{a}$ and b). There are $176 \mathrm{y}$ and $192 u$ output cbannels per chamber. Further details on the PC design and construction are giren bs Markiewicz. ${ }^{30}$

The use of the induced signals allowed a thinner design than would three sense planes plus associated bigh roltage planes. The design also gave a distinct advantage in matching the various coordinates to give points in space. Because the signals on all three planes resulted from the single avalanche on the sense wire, the matching of the planes was independent of the angle of the initial ionizing track. This decoupled the matching process from the track finding process. an immense simplification. The $u$ coordinate was used to resolve ambiguit it : in matching $I$ witb $y$ caused by multiple tracks.

From the amplifier/discriminator cards, signals entered 60-90 w of ribbon cable to bring the signals from different chambers into synchronicity and to allow time for a trigger decision to be made. A trigger signal opened a 70 us write gate. and chamber signals arriving during this interval were latched to await readout by the computer.

The resolution of the PC's was close to the expected $\sigma=($ wire spacing $/ \sqrt{12})$ or $920 \mu \mathrm{m}$ and $1500 \mu \mathrm{m}$ for the sense and induced planes. The sense planes. which measured positions in the magnetic bending direction and thus momentum. were designed to give sufficient position resolution so that the multiple Coulomb scattering (MCS) in the 29 radiation lengtbs between chambers would limit momentum resolution for average length tracks at the highest momenta. The efficiency of the PC's was > $90 \%$ away from the beam. However, at our high intensities the eficiency in the beam suffered, dropping to an average of $83 \%$ and $59 \%$ at beam center for $I$ and $y$. respectively. This was a greater factor in the analysis of multimuon events than in deep inelastic scattering, where the scattered muon rapidly leaves a beam region already obscured by the hadronic shower.

High momentum tracks at large angles can be shorter than average. In order to maintain MCS-limited resolution for these high $Q^{2}$ tracks, th $2 x$ position measurement was augmented by following each PC with a drift chamber (DC). Each DC bad $563 / 4$ inch wide cells with sense wires in the center. A trigger signal initiated a string of 31 time bins during which the ionization electrons drifted to the nearest sense wire. Cp to four occupied time bins could be recorded per cell. A uniform drift velocity would have meant a time bin width of $300 \mu \mathrm{m}$ of $\sigma=90 \mu \mathrm{m}$. In ract, the bulk of the signals arrived within 20 time bins and the velocity w as not esactly uniform. This nonlinear time-distanc a relation was included in the analysis, and the resulting measured position resolution was better than $250 \mu \mathrm{m}$. The effriency of the drift chambers was good, better than $98^{\circ} \mathrm{C}$ in the beam area, but their (31 time bins / $120 \mathrm{MHz}$ clock' meant they were active for 260 s.s or $13 \mathrm{rl}$ buckets. Extra beam tracks were often recorded. These were eliminated, and the left-right ambiguities in the cells were resolved, by referring to the PC system. For further discussion of the drif chamber system, see Ref. 44 . 
MWPC Cathode amplifiers
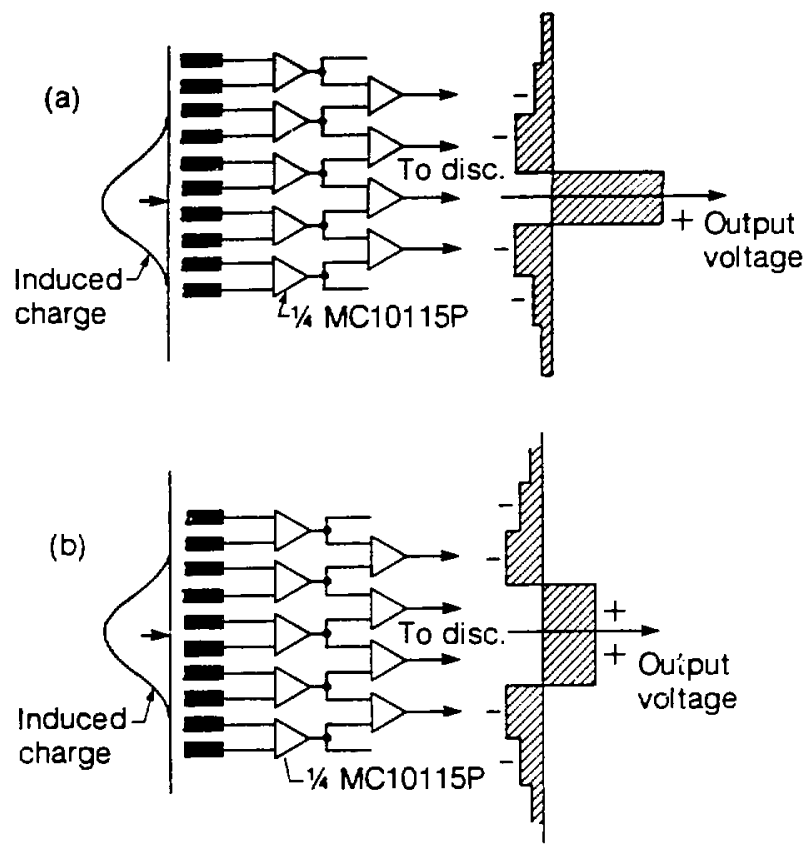

(c)
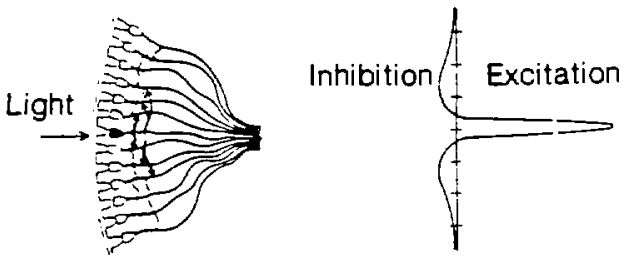

Fig. 4.10. Proportional chamber induced signal readout. The center of the broad input signal is found before a threshold is applied. Altbough there are only balf as many output as input channels, full input resolution is restored by distinguishiug between one (a) and two (b) out put channels abore threshold. (c) Similar circuitry in the eye of the horseshoe crab Limulus (rrom Ref. 42.). 


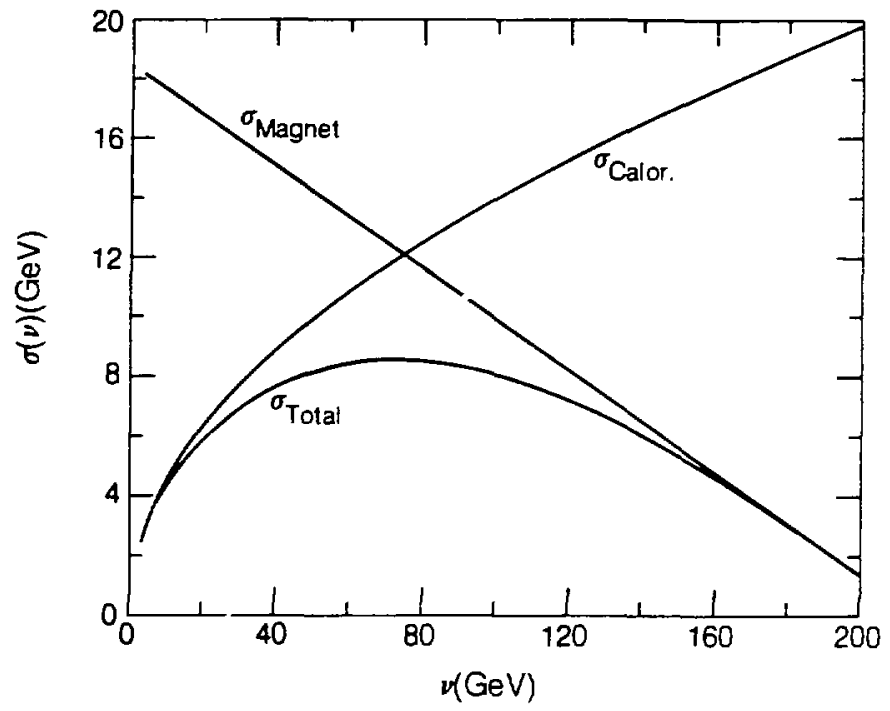

XEL 83:0-731

Fig. 4.11. Calorimeter and magnetic resolution in $\nu$ showing the improrement possible at low $\nu$ using the direct calorimetric measurement.

The arerage momentum resolution for deep inelastic scattering erents in the MMS fas $\sigma_{p^{\prime}} / p^{\prime}=8.6^{\circ} \mathrm{c}$ at a beam energy of $215 \mathrm{GeV}$ and $9.0 \%$ at $93 \mathrm{GeV}$. The average resolution is worse at the lower energy because the tracks are typically shorter.

\subsection{Calorimeter}

Athough the kinematics of an inclasive deep inelastic scattering event can be determined entirely from the initial and final 4-momenta of the scattered muon (Eq. 2.1), there are experimental advantages in measuring $\nu=E-E^{\prime}$ directly by measuring the energy of the hadronic final state. This we do calorimetrically by sampling the hadronic shoxer between the $10.28 \mathrm{~cm}$ iron plates in plastic scintillator. This is useful because, at lor $\nu^{\prime}, E^{\prime}=E-\nu$ with $E$ from the beam system and $\nu$ from the calorimeter gives better resolution than the magnetic determination of $E^{\prime}$. The energy resolution of the calorimeter is $\sigma_{\nu} \approx 1.4 \sqrt{t(\mathrm{GeV})}$. Neglecting the beam momentum resolution, the magnetic and calorimetric resolution of $E^{\prime}$ are equal whed 


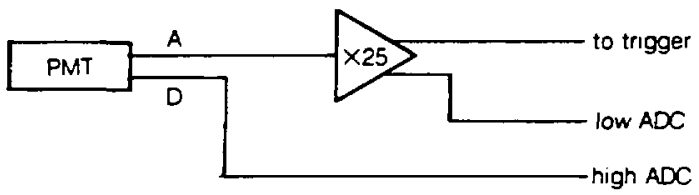

XBL 8310.715

Fig. 4.12. Calorimeter counter readout. The signals on both the anode $(A)$ and the last dynode (D) of the photomaltiplier tube (PMT) are read out to give two orerlapping scales.

$1.4 \sqrt{E-E^{\prime}}=0.086 E^{\prime}$ at a beam energ of $215 \mathrm{GeV}$. The calorimeter thus gives better $E^{\prime}$ resolution for $E^{\prime}>140 \mathrm{GeV}$ or $\nu<75 \mathrm{Ge}^{-1}$ (see Fig. 4.11).

Calorimeter counters were placed to cover the beam area ater each of the first 75 plates, not including plate 0 . A counter is show $\mathrm{a}$ in place in Fig. 4.5. The scincillation light was detected and the resulting signal amplifled at one end of each counter by an RCA 6655 photomultiplier tube. The triangular light guides were made of utraviolet absorbing (UVA) lucite to absorb short wavelength light from the near end of the counter. Short wavelength light has a short attenuation length in the scintillator and cannot reach the phototube from the far end of the counter. The LVA light guide thus makes the response of the counter more spatially uniform. Overall uniformity was achieved by inserting the counters from alternate sides of the spectrometer. The read-out scheme for one counter is shown in Fig. 4.12. The anode signal is amplifed $\times 25$ and sent both to a discriminator for use in the cwo-muon trigger and to a 1024 counc analog-to-digital converter $(A D C)$ for pulse measurement. For larger pulses, the ac signal on the last dynode (about $0.6 \times$ the anode signal) was read directly by a second $A D C$. The amplifled-anode and dynode ADC's (LRS 2249's) were known as the low- and high-ADC's. respectively.

The calorimeter works by sampling the shower produced when the primary hadrons from the event vertes interact in the iron between the counters. A typical shower reaches a masimum in the first or second plate following the verter and estends 5-15 plates, with the mean length depending logarithmically on the shower energy. The individual counters and the calorimeter as a whole were calibrated in terms of "equinlent particles" (EP). the most probable pulse beight produced in one counter by a minimum ionizing particle. This, rather than the mean. is used because the most probable pulse height is independent of the energy of the particle at large energies. ${ }^{45}$ Our source of minimum ionizing particles is. of conrse the muon beam. A 
single muon gives a most probable signal in each low ADC of about 20 counc. The low ADC tbus saturates $a t \approx 50 \mathrm{EP}$. corresponding to the maximum of a $30 \mathrm{GeV}$ shower The high ADC saturates at $\approx 2000$ EP. The analssis of the calorimeter signal is described in Sec. 6 \& Calibration is discussed in Spe . 7.3. 


\title{
Chapter 5 \\ Data Taking and Data Sets
}

\author{
"Dress warm," said the mother, who seemed to koow. \\ Joseph Heller, Cakeh 22
}

\subsection{Operation of the MMS}

The operation of the MUMS was synchronized with the beam cycle through timing signals sent by the accelerator control system. Immediately prior to the one second beam spill, the online computer sent out test pulses and then generated a trigger to record the resulting MLMS signals as in a normal event. During a test event. chamber planes were pulsed and. on alternate spills. either a pedestal level or the response to an LED flash was measured for each calorimeter counter.

During the spill, control of the experiment was handed back and forth between trigger and computer. The more complicated multimuon triggers, notably the dimuon trigger which required input from the calorimeter. necessitated a two-level structure of pretrigger and full trigger. For the deep inelastic trigger. pre- and full trigger were identical. An event which satisfied the deep inelastic trigger generated a pretrigger signal which initiated the latching of lamber information and the digitization of calorimeter pulses and started the drifl chamber clock. The pretrigger signal also inhibited further pretriggers for $3 \mu \mathrm{sec}$. If during that time no confirming full trigger signal arrived. digitization was aborted and the latches cleared. For deep idelastic events, a full trigger always arrived. This signal blocked further triggers for $300 \mu$ sec ind informed the computer that there was an event to record. At this point, the computer took over. ignoring triggers until it was through reading out the chamber systems. the ADC's and the trigger latches $l \approx 1$ msec). At full intensity, typically 50 events rere recorded per spill of which four were deep inelastic triggers. Total deadtime was under $10 \%$. Events were stored in computer memory during the spill, then written to tape between spills.

The beam flux was recorded by simply counting BV signals with a computer-read scaler gated by the same signal as the trigger. It was intecded that this scaler would protide the normalization for all absolute measurements. Unfortunateiy, several supposedly redurdant counts of the lus were found to be al rariance at a level of several per cent. This was traced to an unespected operating mode of one of the 1000 logic elements used in the trigger and meant that the $\mathrm{BV}^{*}$ scaler did not accurately reflect the livetime of the triggers. Fortunately. there ras one count of $\mathrm{BV}^{*}$ that did identically match the trigger livetime. This was the prescaled BV trigger mentioned in Sec. 4.3. When multiplied by the known prescale value, the number of recorded BV 
triggers, which was typically about the same as the number of deep inelastic triggers. provided the beam flux normalization for the experiment.

The rigors of handling and writing to tape 50 events per spill strained the capabilities of our PDP-15 computer to the extent that only minimal on-line analysis was possible. This ras restricted to bistogramming raw information from the MVS to check tbat rarious component. mere operating. A somewhat more detailed diagnostic package was run on randomly selected data tapes several times daily. but we had very little ability to analyze events until after tbo data taking was completed. This was a spvere disadvantage, as was brougbt bome in the late discovery of the trigger counter ineffiency and ot her problems.

\subsection{Chronology}

This experiment was originally advanced in a series of proposals submitted between March 1973 and December 1974. All proposed using rougbly the same apparatus for measurements of rare muon-induced processes with one or more muons in the final state. These were combined into a single experiment with the various processes triggered in parallel and Fermilab Experiment 203/391 was approved in March 1975.

Magnet construction began in Spring 1977. After a period devoted to magnetic measurements. instrumentation of the MMS began at the end of the summer. As installation progressed. low intensity muon running was used to tune up the trigger. Data taking began on 20 January 1978 with all subsystems fully operational by 16 March (after a two week shutdown during a coal strike). Muon running continued until 9 May 1978. During this time 1239 magnetic tapes were Fritred. A typical tape contained one run of approsimately 13000 events, about 1200 of which were deep irelastic triggers. Major analysis began in the summer of 1978 and concluded with production running early in 1980 of the final versions of the track finding and momentum fltting programs on all analyzable data. Results of the multimuon analysis Fere published first. ${ }^{4-49}$ The analysis reported here represents the end of Experiment 203/391.

\subsection{Data sets}

The deep inelastic scattering analysis used a somewhat restricted data sample, corresponding to about $70^{\circ}$ of the analyzable data. Of concern was our ability to simulate and make corrections for data with marginal analyzability or resolution. Runs previously deemed usable were rejected due to various forms of hardware failure, primarily in two classes. The first included data taken whout drift chambers. The drift cbambers contributed to the resolution of the MAIS not only through their superior spatial resolution. but also by filling gaps in tracks due to $P C$ inefficincy. The second class of rejected data included runs where the E98 beam chambers (Fig. 4.3) were operating poorly. The frequent failure of the beam chambers B1 and B2 in front of the MIS made the use of the E98 chambers crucial. Although it was often possible to identify a hit in MMS chamber 0 with the beam track, this was not sufticient to provide an independent measurement of the beam momentum in the CCM unless multiple scattering is the material downstream of the CCM was ignored. The use of measured beam tracks as the parent beam distribution for our simulation required confidence that the measured parameters of recoustructed beam tracks were negligibly different from the true values. For this reason, in the runs retained for analysis, we eliminated erents whese the beam track was missing more than one of the possible four $I$ bits in the E98 chambers (a 10\% Joss) or for which no beam track could be reconstructed at all $\left(8{ }^{\circ}\right)$. Events of the latter type were mostly background. with an off-axis muon entering the MLIS. 
Table 5-I. Data sets. Columns (d)-(g) refer to events with reconstructable beam ' racks.

\begin{tabular}{|c|c|c|c|c|c|c|}
\hline $\begin{array}{c}\text { (a) } \\
\text { Beam }\end{array}$ & $\begin{array}{c}(b) \\
\langle E\rangle \\
\left(\mathrm{GeV}^{\prime}\right)\end{array}$ & $\begin{array}{c}\text { (c) } \\
\text { Intensity } \\
(\mu / \text { spill })\end{array}$ & $\begin{array}{c}\text { (d) } \\
\text { Incident } \mu\end{array}$ & $\begin{array}{c}\text { (e) } \\
\text { Triggers }\end{array}$ & $\begin{array}{c}\text { (f) } \\
\text { Track found }\end{array}$ & $\begin{array}{c}(g) \\
\text { Event flt }\end{array}$ \\
\hline $215 \mathrm{GeV}_{\mu^{+}}^{+}$ & 209. & $3.0 \times 10^{8}$ & $1.91 \times 10^{11}$ & 560872 & 555316 & 531781 \\
\hline $215 \mathrm{GeV}_{\mu}-$ & 209. & $1.0 \times 10^{6}$ & $2.61 \times 10^{10}$ & 58365 & 58110 & 56615 \\
\hline $215 \mathrm{GeV}^{-} \mathrm{tot}$ & 209 . & - & $2.17 \times 10^{1:}$ & $61923 \pi$ & 613456 & 588396 \\
\hline $93 \mathrm{GeV}_{\mu^{+}}$ & 88.0 & $2.5 \times 10^{8}$ & $8.75 \times 10^{9}$ & 66533 & 65740 & 61794 \\
\hline
\end{tabular}

The data sets comprising events with good beam tracks as defined in the previous paragraph are summarized in Table $5-1$. At $215 \mathrm{GeV}$, for both $\mu^{+}$and $\mu^{-}$. roughiy equal amounts of data were taken with each MLIS magnet polarity. At $93 \mathrm{GeV}$. bending the muons to the rest in the MMS was prohibited by radiation-safety considerations and all data were taken with the eastbending magnet polarity. Column (b) show 5 the average energy of beam muons at the interaction vertex. It differs from the nominal beam energy by the average energs loss (1.35 GeV/module at $215 \mathrm{GeV}$ ) suffered by beam muons in the MMS before scattering. Column (c) is the total number of muons penetrating the IMS in the beam area during a spill. Columns (d) and (e) are the totals of incident and scattered muons used in this analysis. Columns (f) and (g) show the faces of these events up to the beginning of physics analysis and will be discussed in the next chapter. 


\section{Chapter 6 \\ Event Reconstruction}

\subsection{Overview}

Reconstruction takes the events observed in the MMS from raw, bardware-produced signals (wire numbers, pulse heights, etc.) recorded on magnetic tape to measured kinematics. This procedure has two major phases: track finding and momentum fltting. In both phases, for reasons that seemed good at the time, an effort was made to treat all types of triggers identically. Track flnding was optimized using trimuon events with the expectation that these would the the most challenging for the programs. As an unfortunate side effect, we did not capitalize upon the simplicity of the deep inelastic events, and their analysis was not hat it might have been. In any case. the track flnding program was quite successful, as can be seen in columns (e) and (f) of Table 5-I.

Event reconstruction proceeds along the following patb:

- Track finding begins wh a preliminary rertex $z$ position which is found by using calorimeter information to locate the beginning of the hadronic shower.

- The beam track is reconstructed.

Hits in ihe beam chambers are used to determine the beam track trajectory and moment um.

The beam track is then projected into the MDIS and PC hits are added. This continues until the preliminary vertex is reached.

- Scattered tracks are reconstructed.

starting from the back of the spectrometer, combinations of hits in tbree chambers are tried until one that gives a sensible moment um and direction is found.

The candidate track is extended a module at a time. Ater each added hit, a new trajectory and momentum are -alculated to project into the next module. This procedure is continued until no hit can be added or the vertes is reached.

New track candidates are tried until all starting combinations up to the vertes are exhausted.

Drift chamber hits are added to each successfully found track. 
- Event fitting starts with a more sophisticated moment um fit of each track. using drift chamber hits when arailable.

- The $x, y$, and $z$ positions of the vertex are determined.

- All tracks are re-fit, this time with all tracks constrained to meet at the common vertex.

- Using information from the calorimeter counters year the vertex, the hadronic energy is calculated.

- The calorimeter measurement of $\nu$ is used to reflne the magnetic determination of $E^{\prime}$.

- Kinematic quantities such as $Q^{2}, \theta$, and $I$ are calculated.

In the following sections of this chapter, these steps are discussed in more detail. A final section deals with the performance of these routines on our data sample. A discussion of the calibration and alignment procedures necessary to the analysis is deferred until the next chapter.

\subsection{Track finciing}

Track flnding was the most difficult part of the analysis of the experiment. The simple geometry of the MMS makes it quite easy for the human eye to identify tracks. Our task was to automate this procedure to bandle the more than 15 million events recorded during the experiment. Unfort unately, pattern recognition is one of the areas where the combination of eye and brain can be vastly superior to machine computation. The track finding task was complicated turther by several factors. some inherent to the distributed-target design of the AMS and some due to imperfections in the instrumencation. In the .AMS, muons travel in nearly solid iron. We must therefore allow for substantial multiple Coulomb scattering and energy loss bet ween position measurements. The energy loss distribution has a tail which estends all the way up to the muon energ: In more than $20^{\circ}$ c of our single-muon events either the beam or scattered track suffered an energ loss of over $5 \mathrm{GeV}$ in a single plate. This energy appeared as an electromagnetic shower, fouling the calorimeter and chambers. Delta rays $w$ hich escape into a chamber without showering can degrade track-finding by giving signals on wires adjacent to the one hit by the muon. These complications are compounded by instrumental effects, primarily the livetime and inefliciencs of the proportional chambers. The 70 ns PC write window extended beyond the \pm 1 rf-bucket beam veto. This permitied the recording of out-of-time "ghost tracks, $\approx 20 \% \mathrm{c}$ of which showered.

The ineflleiency was a more serious problem. To allow for missing hits, it was necessary to allow projection through more than one module in extending a candidate track. The momentum uncertainty (initially very large) and multiple Coulomb scattering then demanded largr search windows, increasing the probability of picking an incorrect hit to add to the track. Inclusion of a nearby wrong hit was often suff cient to derail the extension of the t.rack. Figure 8.9a shows a typical deep inelastic scattering event. Alebough its overall pattern is unambiguous, there is clearly a fair amount of electromagnetic hash obscuring the tracks.

The track finding algorithm was basically a brute sar-p trying of combinations of hits. Cleverness and endless testing were applied primarily toward reducing the number of avajable combinations. During the testing. a tremendous number of decisions of no general interest. but of crucial importance to the track tinder, had to be made. Such questions as how many consecutire miscing chambers 10 allow in a track had to be answered empirically. Our solutirns to these questions are described in Ref. 38. For completeness. I will describe the general orerution of the 
track finder and discuss its limitations, which were most apparent in the attempt to measure precisely the low-background deep inelastic signal.

The first step in track finding was to convert the raw list of hit wires into spatial coordinates. To do this, hits on adjacent wires due to, sag. a muon plus a $b$-ray or small shower were first coalesced into a single hit. The position of the new hit was deflaed to be the center of the group of hit wires and the width set equal to the (number of hit wires/ $x$ (changel spacing) This redefinition of the width is incompatible with the output scheme of the induced electronics. which half the time gare two adjacent hits for single tracks. Although the result was to inflate mistakenly the error on some of the $y$ positions by a factor of $t$ wo. the central position was calculated correctly. Studies showed no significant degradation of angular resolution due to this problem. but the $x^{2}$ 's for our fits of trajectories in the $y$ view come out unnat urally small.

These redefined hits are then used in the matching of the independently recorded $x, y$. and $u$ coordinates into "triplets," each with a unique $x-y$ position. The poor efficiency of the PC induced planes required the retention of unmatched hits as "singlets." also arailable for use on tracks. Triplets. which were needed to tie the $I$ and $y$ views of the tracks together, were given higher priority in the reconstruction.

The search for bits to attach to beam and scattered tracks was limited along the beam direction by a preliminary vertex $z$ position found using individual calorimeter pulse heights to locate the beginning of the hadronic shower. The determination of this vertex was begun by finding the largest single pulse height. In large hadronic showers, the mean location of the shower maximum occurs after more than $10 \mathrm{~cm}$ of iron. More important. hadronic showers have notorious fluctuations. It was therefore necessary to look upstream of the maximum to find the beginning of the shower. With $A$ the maximum pulse height, a threshold of 0.08 .4 was chosen empirically 10 define shower activity. To avoid missing the beginning of a shower with a large downward tuctuation in pulse height before the maximum and also to avoid incorporating electromagnetic splashes from the beam track into the shower, all pulse heights upstream of the maximum were cumpared to the threshold. The verte $\mathbf{x}$ as placed in the middle of the plate that maximized $\therefore=($ the number of pulse heights $<0.08 .1$ ) - (the number of pulse beights $>0.08 .4$ ) upstream of the vertes. The operation of this algorithm is illustrated in Fig. 6.1.

The most frequent failure of the calorimeter vertex finder occurred when the larges single pulse height was due to an electromagnetic shower away from the true vertex. Electromagnetic showers give more pulse height per GeV than hadronic showers. They are aiso shorter. and thus can bave very large maxima. When the rertex finder was fooled by an electromagnetic shower. either the beam or sizitered track finding was stopped short. Even if the verter was mored to the proper location turing fltting (see the nest section), the event was sometimes rejected for having a large gap in the track. Approximately $0.6^{r}\left(1,3_{c}^{r}\right)$ of the $215(93) \mathrm{GeV}^{\circ}$ events were lost in this way.

With the calorimeter vertex dividing the event into two sections, track finding proceeded with the separate reconstruetion of the beam and scattered tracks. The beam track was begun in the beam chambers upstream of the MMS. It momentum and trajectory in $I$ and $y$ (including. if possible. the scattering in the lead glass) were fitted. The track was then projected to the front of the IMIs and the momentum was corrected for energy loss in the lead glass and ot ber material in the beam. From there. the beam track was extended. one module at a time, by using the track as reconstructed up to that point to predict a central position in the next proportional chamber. The position. angle, and momentum uncertainties for the track and the predicted magnitude of multiple scattering were used to open a search window. A PC bit within the window was added to tbe track. and this was coutinued until the last chamber before the vertex. Only one beam track ras sought. 
(d)

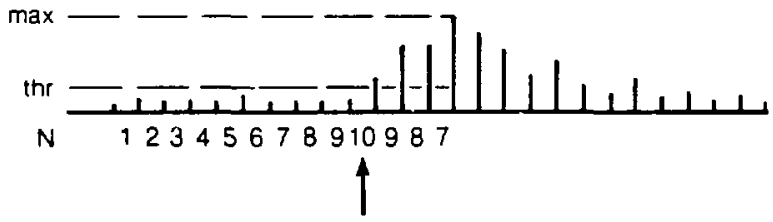

))
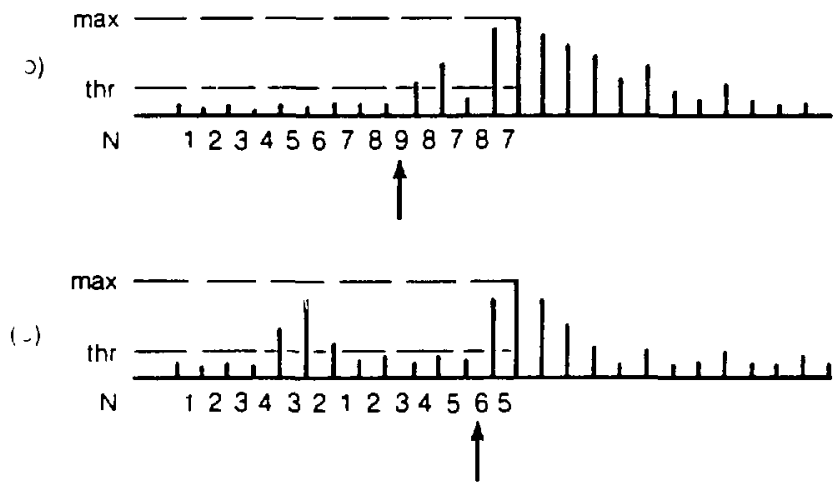

XEL 8310-717

Fig. 6.1. The calorimeter vertex-flnding algorithm. The rertical bars indicate 'ie pulse heights in individual counters. Each pulse height is compared to a threshold of 0.08 times the maximum pulse height. The result for each counter of the algorithon discussed in the text is shown as $N$. The vertes is assigned to the plate following the maximum of $V$ (arrow). The point of the algorit hm ie in allow for Auctuations before the shower maximum (b). without including separate electromagnetic showers (c). 
From the back of the spectrometer, starting combinations of three triplets or two triplets plus an unmatched $z$ and $y$ were investigated. One empty chamber was allowed between hits. but the combination had to meet angle and linearity requirements in $y$ and angle and minimum momentum requirements in $x$. Valid starting combinations mere pursued upstream modnle by module in the same fashion as for the beam track until the vertex was reached. The track was then projected to pick up bits downstream of the starting segment. Accepted tracks bad $x$ and $y$ hits in at least. four chambers, separated by no more than two consecutive empty chambers. At least tro of the $(x, y)$ pairs were required to be triplets. All possible starting combinations made up from hits downstream of the calorimeter vertex were investigated. All ralid tracks of either charge were retained.

For all valid tracks, including the beam track, drift chamber bits were added aher track finding was complete. In each drift chamber. the two bits closest to the track position were saved. These two hits could be the left-right ambiguous options of the same hit. The choice of which to use was postponed ur:il a better fit of the trajectory could be performed.

\subsection{Momentum fitting}

Our momentum fitting algorithm took the measured positions of a found track and calculated the 3 -momentum of the track at some reference point (usually the vertex) and a detailed trajectory of the track through the spectrometei. The calculated trajectory included the effects of magnetic defection, energy loss, and multiple Coulomb scattering (MCS). The magnetic deflection was treated as a single transverse impulse between position measurements. It used the detailed field map produced during the MMS ca! 'bration, whi:b included the small $x$ component of the field. Energy loss was included as a continuous correction to the muon energy along the track. For a muon of energy $\mathrm{E}$ ( $\mathrm{GeV}$ ), the energy loss in $\mathrm{MeV} /\left(\mathrm{g}^{-\mathrm{cm}^{-2}}\right.$ ) is

$$
\frac{d E}{d x}=1.825+0.0716 \ln \left(\frac{E^{2}}{E+10.9}\right)+0.0045 E .
$$

Besides the ionization loss, this expression includes terms due to $\delta$-rays, bremsstrahlung, and pair production. The terms for these processes, which do not occur smoothly, represent averages integrated uf o same maximum allowed energy lass. It is presumed that energy losses larger than this maximum would disrupt the track and confound the track finder. and thus would not appear within a single found track. MCS is handled by actually fitting the residual deflections between chambers after removing those due to the magnetic field.

Three variations of the fitting routine were used. These were applied to the $x$ view of scattered tracks, the $y$ view of scattered tracks, and either view of beam tracks. The first of these. the bending view, was the most general. For a track with $N$ transyerse position measurements $x_{1} \pm \sigma_{1}$ at $z_{1} \quad(i=1, \ldots, N)$, we define $a_{0}, a_{1}$, and $a_{2}$ as the $x$ position. slope, and inverse momentum at the reference point $z_{0}$. The predicted positions $\xi_{i}$ of the track are then ${ }^{50}$

$$
\xi_{1}=a_{0}+a_{1}\left(z_{1}-z_{0}\right)+\sum_{j=1}^{i-1} a_{2} g_{j}\left(z_{1}-w_{j}\right)+\sum_{j=1}^{i-1} a_{2} p_{j}\left(z_{1}-w_{j}\right)
$$

Bere the $q$, are the unknown MCS $p_{\perp}$ 's that occur at $w$, between $z_{j}$ and $z_{j+1}$, the $p$, are the known magnetic deflection $p_{\perp}$ 's, and, for demonstration purposes, energy loss has been neglected. In this expression there are $\mathcal{N}$ equations corresponding to the $\because$ measurements and $X+2$ (three $a$ 's and $X-1 q$ s) unknowns. This distasteful situation is rectified by the constraint 
that the average MCS $p_{\perp}$ is zero. This constraint is applied by adding $N-1$-measurements" $q=0$ with a "measurement uncertainty" equal to the expected rms ralue of the MCS $p_{1}$. $\delta=15 \sqrt{\mathrm{X}} \mathrm{MeV} / \mathrm{c}$, with $X$ the path length in radiation lengths. The $\chi^{2}$ of the fit is then

$$
\chi^{2}=\sum_{i=!}^{N} \frac{\left(x_{1}-\xi_{1}\right)^{2}}{\sigma_{1}^{2}}+\sum_{j=1}^{N-3} \frac{q_{j}^{2}}{\delta^{2}} .
$$

The value of $\chi^{2}$ is minimized to give the best values of the fit parameters $a_{0}, a_{1}, a_{2}$ and $q_{1} \ldots, N-1$ For the $y$ view of scattered tracks, $a_{2}$, the inverse momentum. was not fitted. Instead, the value from the $x$ fit was used. For beam tracks, both the invirse momentum $a_{2}$ and the slope $a_{1}$ were fired at the front of the spectrometer to the values determined with higher resolution in the beam system. in each fit, the routine was permitted to discard a limited number of hits if the $x^{2}$ was poor.

Each track was jtted several times in the course of fitting an event. In the first fit, only proportional chamber hits were used. U'sing the initial fitted trajectory as a guide, the $x$ vien of each track was refitted, this time with the routine choosing the best hit in each chamber from the one PC and two DC hits provided by the track finder. If the track finder got confused, it Fas common for it to find two or more segments of a long track and call them separate tracks.

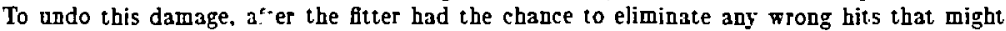
have misdirected the track finder, an attempt was made to rejoin tracks that appeared similar. Successful matches were consecrated by refitting them as one track. At this time, "ghost trach"s" made of hits due to out-of-time muons were eliminated. This was done by requiring that the trigger counters, which had output pulse lengt hs less than the width of an rf bucket, register the passage of all fitted tracks.

The next step in event fitting was to require that all tracks intersect at a common vertes. The position of this vertex was determined by using the preliminary (calorimeter) vertes and the tracks themselves. For each outgoing track, the point of closest approach to the beam track was calculated. Tracks with too large an impact parameter were eliminated. The vertes was constructed from the remaining tracks and the calorimeter vertes by finding the $z$ position which minimized

$$
\chi^{2}=\sum_{1} \frac{\left[x_{1}(z)-x_{b}(z)\right]^{2}}{\sigma_{x}^{2}}+\sum_{i} \frac{\left[y_{i}(z)-y_{b}(z)\right]^{2}}{\sigma_{y}^{2}}+\frac{\left(z-z_{\text {ealorimeter }}\right)^{2}}{\sigma_{\text {ealorimeler }}^{2}}
$$

where $i$ refers to outgoing tracks, $b$ refers to the beam track, and $\sigma_{\mathrm{cs} \text { orimeter }}$ is the estimated

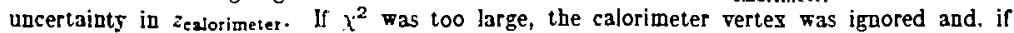
necessary, tracks were eliminaced. The $x$ and $y$ positions of the vertex were then the weighted averages of the positions of the surviving tracks at zuertex. All tracks were then refit with the new requirement that they pass through the rertex. Tracks which could be successfully fit in this fashion were called "vertes enabled." The distribution of vertes $z$ positions from our $215 \mathrm{GeV}$ data set is show $\mathrm{n}$ in Fig. 6.2. Note that the large gaps between modules are easily resolved. 


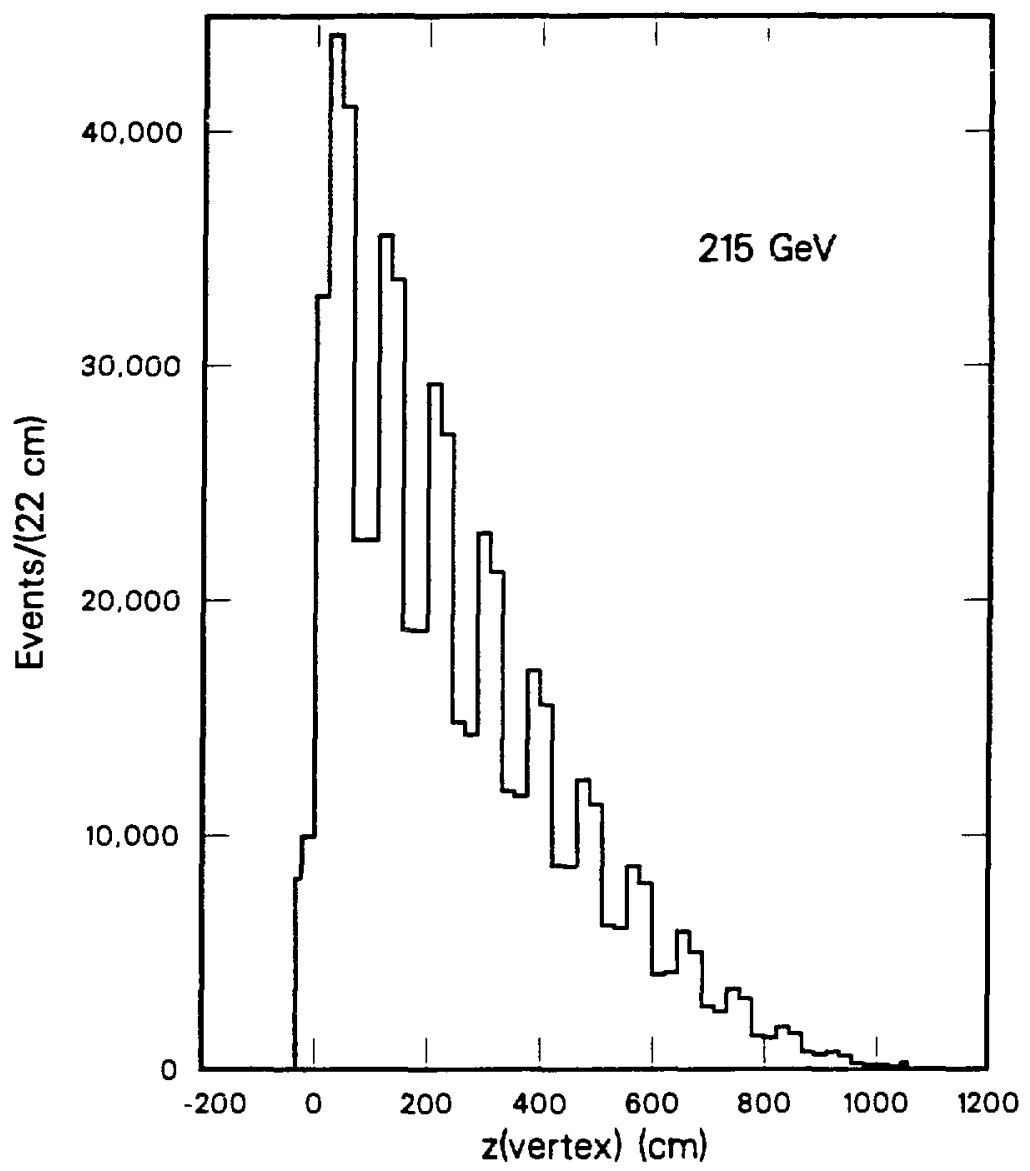

XCG $8311-7277$

Fig. 6.2. The distribution of event vertices along the beam direction for the $215 \mathrm{GeV}$ data. 


\subsection{Calorimetry}

Once the vertex was located, the pedestal-subtracted signals (calibrated in equiralent particles, see nest chapter) from calorimeter counters in the surtonnding region were summed to give a direct measurement of $\nu$, the energy of the hadronic final state. The summed region started five counters upstream of the estimated vertes position and extended to ten counters downtream of the rertex. This interval was extended if the counters at the ends shoned more pulse height than expected from a single muon. In the sum, each counter signal bad subtracted from it the mean pulse height from a single muon $1 \approx 2.6 \mathrm{EP}$. This summed pulse beight tras turned into $\mathrm{GeV}$ via the calibration procedure described in the nest chapter. The conrersion was approximately $6 \mathrm{EP} / \mathrm{GeV}$.

With $\nu$ determined independently, we could measure the missing energy of events. $E_{\text {miss }}=E-E^{\prime}-\nu$ (Fig. 6.3). For deep inelastic scattering events, this was due entirely to instrumental resolution. As discussed in Sec. 4.7, the ot herwise redundant calorimeter information can be used to improve the $E^{\prime}$ resolution of individual tracks. The procedure assumed that the resolution on the beam energy measurement was negligible. $E^{\prime}$, previously determined from the momentum fit. nas redefined to be the weighted average of the original value and $E-t$. The individual components of $\mathrm{p}^{\prime}$ xere then adjusted using the correlations determined in the momentum ft. Events ith large $\left|E_{\text {miss }}\right|$ were suspect. Large positive $E_{\text {miss }}$ could mean that the shower was missed completely. Large negative $E_{\text {miss }}$ could accompany a wide angle bremsstrahlung event with an electromagnetic shower (see Appendis $A$ ). For this reason. the calorimeter information was used only in events with $\left|E_{\text {miss }}\right|<02$ (26) GeV for the 215 (93) Get data sets. These cuts correspond to approsimately $2.5-3 \sigma$ in $E_{\text {miss }}$, independent of $E^{\prime}$.

\subsection{Performance}

Table 5-1, columns (e). (f). and (g) summarize ous success in reconstructing events. All entries refer to events with successfully reconstructed beam tracks. For the $215 / 93 / \mathrm{GeV}$ dat 3 sets, the scattered track was found in $99.1 \%(98.8 \% \mathrm{c})$ of the events. Of these events. $95.9 \sigma_{c}$ $\left(94.0{ }^{-}\right)$were successfully moment um- and vertex-ftted. Losses through this stage of the analysis

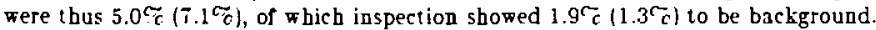

Momentam resolution in the MMS was limited by multiple Coulomb scattering to about $8 \%$. The resolution for short, low momentum tracks was somew bat worse - about $10^{\circ} \tau$. Using the calorimeter improved the resolution at high $E^{\prime}$ dramatically. In the $215 \mathrm{GeT}^{\prime}$ data. the resolution for $E^{\prime}>150 \mathrm{GeV}$ without the calorimeter was $\sigma_{E^{\prime}}=0.08 E^{\prime}$. Using the calorimeter improved the resolution to $\sigma_{E^{\prime}}=0.05 E^{\prime}$. Figure 6.4 shows our resolution in $Q^{2}$ and $I$ at various locations in the $Q^{2}-x$ plane. The inner and outer bars indicate $\sigma$ with and without calorimetry. The largest improvement occurs at low $\nu$, that is, the lowest $Q^{2}$ for each ralue of $I$. This Gigure includes the effects of radiative corrections which, in effect, change the internal kinematics of an erent without changing its appearance to the outside world. The $Q^{2}$ resolution ras roughly constant at $13{ }^{\%} \mathrm{c}$. The $I$ resolution varied between $133^{\sigma_{C}}$ and $30^{\sigma_{c}}$.

These ralues for the resolution come from a program which simulates deep inelastic scatcriog events in the MMS. At $215 \mathrm{GeV}$, the width $(\pi)$ of the distribution of ( $\left.E_{\text {mesured }}^{\prime}-E_{\text {genersted }}^{\prime}\right) / E_{\text {generated }}^{\prime}$ is between 0.074 and 0.083 . depending on how much of the rail is included. Another estimate of the resolution comes from the momentum uncertanty calculated by the atting routine, combined with the calorimeter resolution. For the same simulated events. the mean uncertainty is 0.077 . This is a useful quantity because it can also be calculated for real events. where the result is 0.076 . 


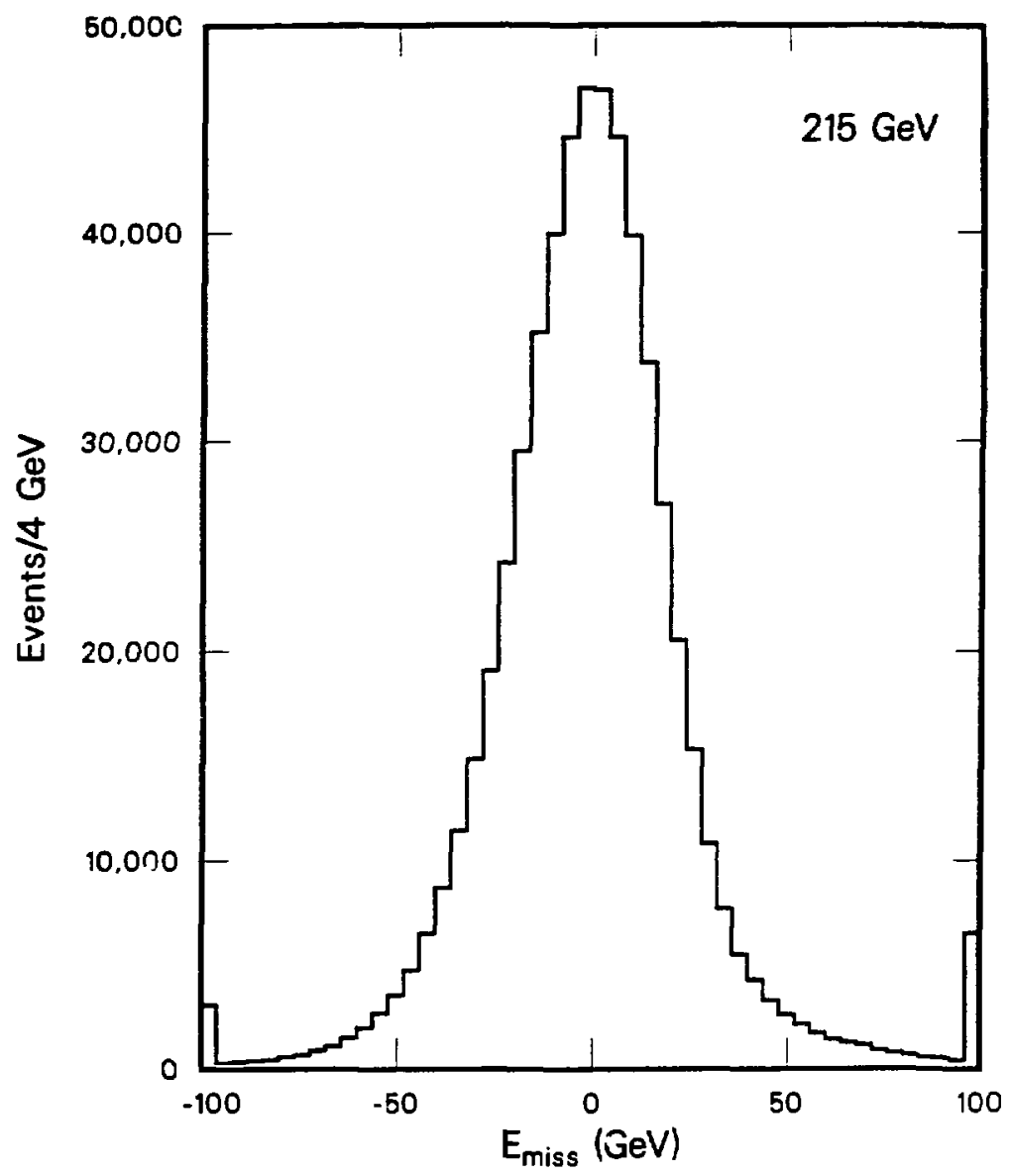

XCG $8311-7278$

Fig. 6.3. The missing exerg distribution of the $215 \mathrm{GeV}$ data. The mean is calibrated to be zero, and the width $\left(\sigma=21 \mathrm{GeV}^{\mathrm{N}}\right)$ is consistent with our expected beam. momentum, and calorimeter resolution. 


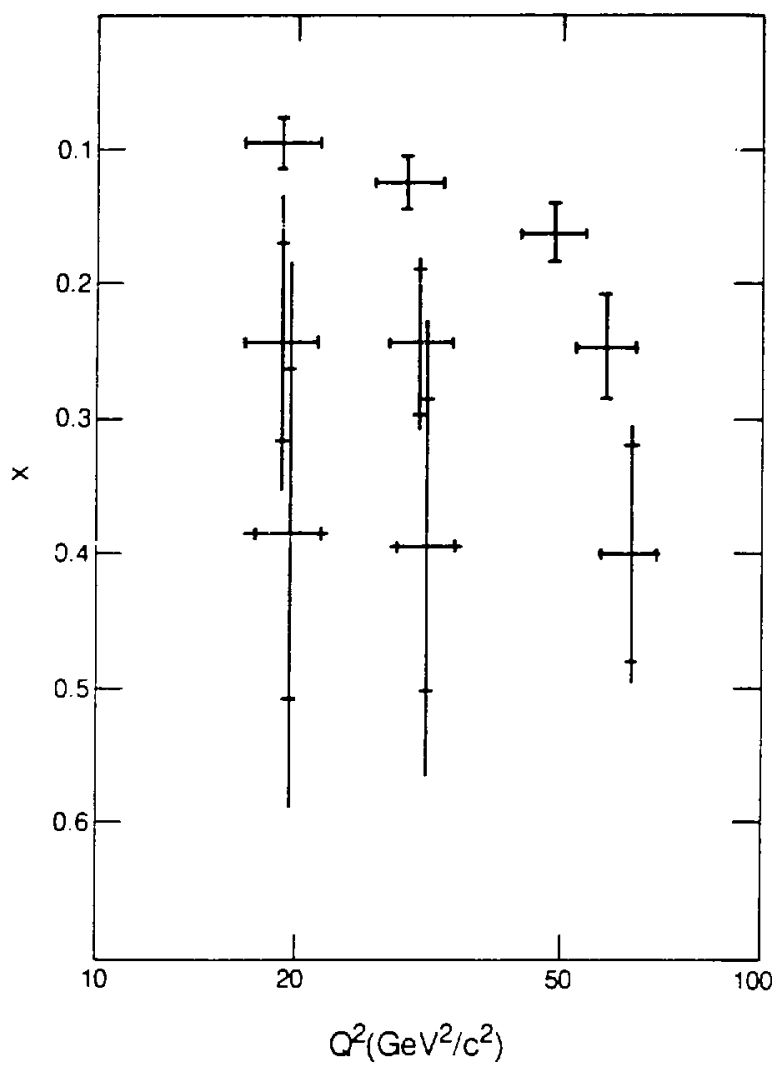

XBL 8310.735

Fig. 6.4. MLS resolution in $I$ and $Q^{2}$. The inner and outer bars indicate the resolution $( \pm \sigma)$ with and without the use of the calorimeter. Note that the origin in $I$ is at the top. 
The results of the data reduction are shown in Fig. 6.5. Where the measured spectra of reconstructed erents in $Q^{2}$. 3 . and $E^{\prime}$ are plotted for both the 215 and $93 \mathrm{GeV}$ data samples. The production running of the event reconstruction routines on our large amount of data xas an arduous task that was performed only once on the full set of analyzable data. The effects of minor mistakes found after that point were corrected by subjecting the simulared events used in the acceptance caleulation to the same errors in reconstruction. 

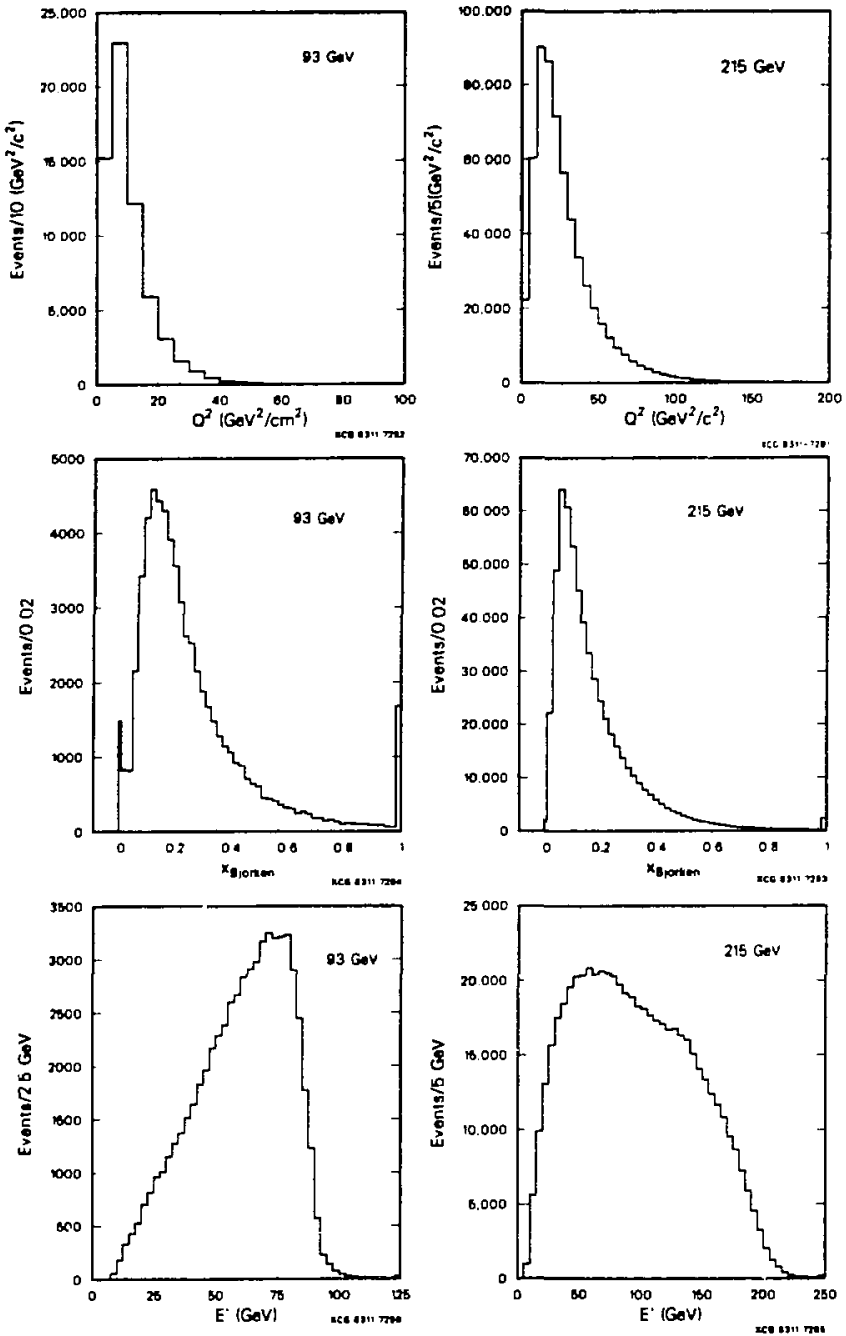

Fig. 6.5. Spectra of all reconstructed events in $Q^{2}, I$, and $E^{\prime}$. 


\section{Chapter 7 \\ Alignment and Calibration}

In this chapter I discuss the various auxiliary measurements necessary to event reconstruction and analysis. This discussion follows that of reconstruction because of the iterative nature of most of these calculations. Although this information is used as input to the reconstruction and simulation programs, it was often derived using these same programs.

\subsection{Chamber alignment}

Surveying instruments were nsed to align both proportional and drift chambers in the MMS to an estimated $130 \mu \mathrm{m}$ accuracy. The aligrment was checked and improved during data reduction by nsing muon trajectories from BV triggers to determine alignment constants, offsets to be added to raw coordinates in each chamber. This task was complicated by three factors: 1) the rms multiple scattering of $180 \mu \mathrm{m} / \operatorname{module}$ at $215 \mathrm{GeV}, 2$ ) the large residual magnetic fleld $\left(\approx \frac{1}{2} \times\right.$ full field $), 3$ ) the lack of confdence in the knowledge of the beam system alignment at that time. These factors meant that: 1) the average of many tracks had to be used, 2) the tracks curved (we left the magnet. at full fleld), and 3 ) the momentum which determined the curvature was unknown.

The alignment procedure was to tit a large sample of tracks, ignoring the information from a single chamber. The initial position, angle, and momentum of the track were determined from the fit. The atted position of the track at the ignored chamber was compared to the position of the hit in that chamber. An offet was then added to the chamber position to make the mean residual equal to zero. This was repeated for each chamber. and the whole procedure was iterated to produce a set of self-consistent alignment constants. The drift chambers were aligned to about $80 \mu \mathrm{m}$ accuracy for the event reconstruction. After the production running. the DC offsets were remeasured to $20 \mu \mathrm{m}$ and the se locations were put into the apparatus simulation. the events from which were reconstructed with the same set of constants used on the real daia.

With the position, angle, and curvature (momentum) of the tracks left free in the flt, constant systematic oflsets in alignment or those that go as $z$ or $z^{2}$ are not detectable in the residuals. The assumption used in the alignment procedure was that there were no such effects and the mean oflset, angle. and curvature of the alignment constants was zero. Fet it is precisely the last of these terms that can systematically affect the momentum measurement. In fact, after the initial alignment, it was found that the average fltted momenta for east- and west-bending $215 \mathrm{GeV}^{\circ}$ tracks difiered by $1.06 \mathrm{GeV}$, indicating that a flnite curvature did exist in the alignment. 
corresponding to a radius of curvature of 214 miles. A quadratic correction was thus applied to the drift chamber cfisets. This correction was everywhere less than $60 \mu \mathrm{m}$.

The horizontal positions of the top and bottom of each chamber were surreyed separately. Thus the possibility that the chambers were rotated about their centers had to be investigat ed. A comparison of residuals for tracks scattered up and down in the MDIS showed indications of random rotations at the level espected from the surveying precision. Fortunately, the effects of such rotations on the fitted momentum are negligible for deep inelastic scattering event $s$. To produce a systematic effect on the momentum, an offset which is quadratic in $z$ is required. A systematic rotation (due to systematic effects in the survey or to chance) that raries linearly with 2 will combine with the vertical scattering angle to give such an effect. If the chanber rotation is $\alpha(z)=a z$, and $\theta$ is the scattering angle of a track, the chamber offet seen by the irack is

$$
\delta(z)=\alpha(z) y(z)=a \theta z^{2} .
$$

This effect might manifest itself as a difference in the fitted momentum betwecn bigh moment um tracks scattering up and down at large angles. High momentum is required to give the track a curvature smail enough so that the chamber ofset is noticeable. The kinemati. limit $Q^{2} \leq$ $2 \mathrm{H}_{N}{ }^{\prime}$ (or Bjorken $x \leq 1$ ) gives a maximum scattering angle that varies inversely with $E^{\prime}$. Given our survey precision, this restricts the possible systematic effect on the momentum at $215 \mathrm{GeV}$ to less than $0.1 \vec{c}$.

\subsection{Magnetic field integrals}

The absolute calibration of the MMS magnet was described in Sec. 4.4. In the beam system, both the last dipole (actually three magnets labeled D4 in Fig. 4.2) and the Chicago Cyclotron Magnet (CCM) were used for the moment um measurement of beam muons and had to be calibrated. The fleld integrals of the D4 and CCM magnets were numerically integrated from flip cuil measurements of the field along the patb of the beam. At $215 \mathrm{GeV}$, the D 4 magnets were operated at 4515 amp, giving a fleld integral of $205.9 \mathrm{kG}-\mathrm{m}$. The CCM field integral at 3100 amp was $59.70 \mathrm{kG}-\mathrm{m}$. At $93 \mathrm{GeV}$, the field integrals were $88.84 \mathrm{kG}-\mathrm{m}$ and $29.60 \mathrm{kG}-\mathrm{m}$.

The beam chambers were aligned in a similar rashion to those in the spectrometer. Hеге we were aided by the fact that some of the chambers were on a direct unobstructed line and could be aligned with magnets off and no bending. With both the bean and spectrometer aligged and calibrated, BV triggers could be used to compare the two systems. BV triggers were treated differently from deep inelastic triggers by the track finder. No calorimeter vertex was found for $\mathrm{BV}$ triggers. Instead, the beam track was allowed to continue to the back of the spectrometer. Then a "scattered" track was found from the back ir. :sc usidi fashion and allowed to continue to the front. When these two versions of the sane track were fit. two values of the momentum at cbamber 0 mere produced, the first from the benm system. the second from the MMS. Ising equal amounts of $215 \mathrm{GeV}$ east- and west-bending MLS data, a discrepancy of $p_{\text {beam }}-p_{\text {ans }}=2.39 \mathrm{GeV} / c$ was found, a value that was constant tbroughout the experiment . This $1.1 r_{c}$ difference was attributed to calibration or alignment errors in the beam system. There were two pieces of evidence backing this interpretation. The first was the estimated error in the MIMS magnet calibration of $0.2{ }^{2}$. This was confirmed at a level smaller than $1 \sigma_{c}$ by our measurement of the 2 mass using muon pairs in the $\mathbf{M M S}{ }^{30}$ For the elastic, inelastic, and total $t^{\prime \prime}$ samples, the differnces between the measured and true mass were $0.7 \tau_{0},-0.9 \tau^{\circ}$, and $0.2 \sigma^{-}$. with statistical errors of about $0.2^{5}$. We thus applied -2.39 and $-1.67 \mathrm{GeV} / \mathrm{c}$ corrections to the measured momenta of individual beam tracks in the beam system for the 215 and $93 \mathrm{GeV} / \mathrm{C}$ beams. 


\subsection{Calorimeter calibration}

The calibration of the calorimeter bad three distinct phases. The first was an ADC counts to equivalent particles (EP) calibration which gave the 75 counters 3 common scale in the low ADC's. Nest was a bigh ADC to low ADC calibration to match the two scales in each counter. Last was an EP to $\mathrm{GeV}$ calibration of the summed counters in a shower.

Tsing the definition of EP, the single muon pulse height spectrum for each counter was inspected by eye to determine the location of the peak, after pedestal subtraction. As an (apparently cursory) study revealed no time rariation of this calibration. only one set of constants was used. Single muons gave no appreciable signal in the high ADC"s. The bigh scale was calibrated by comparing large low $\mathrm{ADC}$ signals from showers ith their high ADC counterparts. I wo problems arose, botb worsening with increasing intensity: the relation between high and Iow broadened with intensity, and events with a random high/low ratio appeared at the few percent level at the tighest intensities. With no further information to go on, these problems were ignored and low intensity data was used to ft (high) $=a($ low $)+b$.

The energy calibration was performed in two steps, each of which compared the summed calorimeter signal to $\nu_{\text {mag }}$, the value of the shower energy meaiured magnetically as $E-E^{\prime}$. The first calibration simply flt $S=c \nu_{\text {mag }}$ where $S$ is the sum of calorimeter signals in EP described in Sec. 6.4. The value found for $c$ was $5.965 \mathrm{EP} / \mathrm{GeV}$. This linear fit was adequate only as a starting point. Problems with it included a possible offset due to inadequacies in the muon pulse height subtraction and an observed anti-saturation (more than $5.965 \mathrm{EP} / \mathrm{GeV}$ ) at large $\nu$. The muon subtraction used the mean pulse height for $215 \mathrm{GeV}$ muons. Unlike the most probable pulse height, the mean is energy dependent, and a correct subtraction should depend on $\nu$. The anti-saturation is believed to be due to radiative corrections. especially wide angle bremsstrahlung events, which add a large pulse height electromagnetic component to the showers and which trigger the MMS only at large $\nu$. The flnal energy calibration was also the catch-all for curing the deficiencies in the previous calibration stages.

The final calibration was a correction derived from another comparison of $\nu_{\text {calor }}$ with $\nu_{\text {mag }}$, which, by definition, has its worst resolution where we need the calorimeter the most. At low $\nu$ (or high $E^{\prime}$, see Fig. $6.5 \mathrm{c}$ ) the spectrum of triggered events drops off rapidly. Thus a bin of low ineasured $\nu_{\text {mag }}$ bas $\left.\left\langle\nu_{\text {true }}\right\rangle\right\rangle\left\langle\nu_{\text {mag }}\right\rangle$ due to smearing. This bias must be removed from the calorimeter calibration. This was done using the apparat us simulation (see next chapter) wbich produced events with known values of botb $\nu_{\text {true }}$ and $\nu_{\text {mag }}$.

Before proceeding with the calibration, an important correction was made. The calorimeter, like the chambers, had a write gate that extended over several rf buckets. Since a muon contributes a mean pulse height of several $E P$ in each of the $\geq 15$ summed counters in a shower, 1 muon from another if bucket could add several GeV to the apparent shower energy. Fort unately, this effect decreased ith the numher of buckets separating the ghost muon from the triggering muon. We vetoed buckets \pm 1 and tagged buckets $\pm 2,3$. To events with such a tag, a correction was applied. The largest correction was $-5.6 \mathrm{GeV}$ for bucket +2 in the $215 \mathrm{GeV}$ data.

The final calorimeter calibration began with sampies of real and simulated data, each divided into bins of measured $\nu_{\text {mag }}$. For each bin. we produced histograms of $v_{\text {calor }}$ for the real data and $l^{\prime}$ rea, the true value of $E-E^{\prime}$, for the simulated data. We then plotted $\left\langle\nu_{\mathrm{cs}} \mathrm{los}_{\mathrm{s}}\right\rangle-\left({ }^{\prime} \mathrm{gen}\right\rangle$ os. $\left\langle\nu_{\text {gen }}\right\rangle$. One such plot is shown in Fig. 7.1 . These points were then fir, using a fourthor sixth-order polynomial with only eren porers of $\left(\nu_{\mathrm{B} \text { ва }}\right)$. The ft was then used to correct the original linear calibration. Inspection of the calibration plots for different blocks of data shored significant time dependence. Much of this was found to be synchronized with the feld reversals of the MMS, an effect traced to magnetic fleld sensitivity in individual photomultiplier 


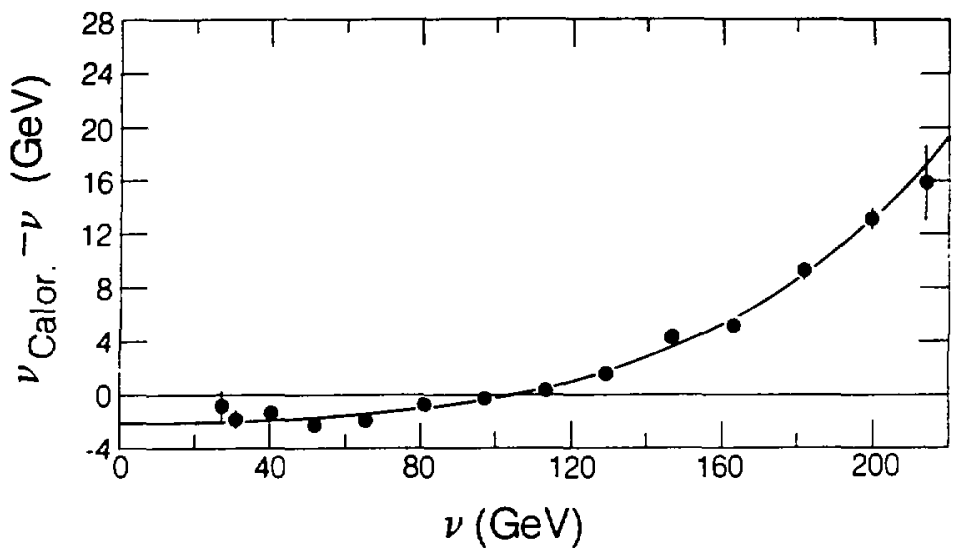

XBL 6310-727

Fig. 7.1. Typical calorimeter final calibration plot. Lncorrected $\nu$ (calorimeter)$\nu$ (truel is plotted vs. $\nu$ (true) determined bin by bin using our Monte Carjo simulation. The carve is the fit to be subtracted from the uncorrected measurements to give the final calorimeter energy.

Lubes. These effects were corrected in an average sense only by separately calibrating blocks of data. each spanning only one AMtS polarity. We also looked for the effects of transverse shower containment on the calibration. Fortunately. large shower recoil angles are correlated with small shower energies and thus shorter showers. A look at the lenst favorable combinations

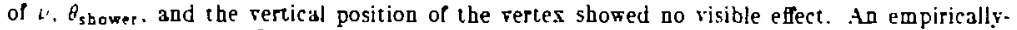
motirated search for $Q^{2}$ dependence showed an effect at large " (see Fig. T.2). beyond where the calorimeter contributes to the $"$ resolution. This effect, visible aiso in the simulated data sample, is due to radiative events which occur preferentially at large 2 and low $Q^{2}$.

By methods similar to those used in the calibration. we determined the resolution of the calorimeter. Ising the same binned dat a, we us ${ }^{\circ} d$ the widths of the histograms to compute

$$
\sigma_{\text {edat }}^{2}=\sigma_{\nu_{\text {calor }}}^{2}-\sigma_{\nu_{\text {gen }}^{2}}^{2}
$$

with $\sigma_{\nu_{C} \text { alor }}$ and $\sigma_{\nu_{\mathrm{K}^{n}} \mathrm{f}}$ from the histograms. The results are plotted as $\sigma_{\text {ealor }} / \sqrt{l_{\text {gen }}} u s$. $\left\langle l_{\text {gen }}\right\rangle$ in Fig. 7.3. This procedure as rery susceptible to disruption by tails in the histograms. For this reason, in Fig. 7.3 we show also for comparison $\sigma_{\text {cslor }}$ estracted by this method from simulated data generated with $\sigma_{\text {ealor }}=1.5 \sqrt{\text { मfGell }}$. From measurements sucb as this we estimate that $\sigma_{\text {(a) } t h} / \sqrt{t^{\prime}}=1.35$ 10 1.7 ar $\iota^{\prime} \leq 80 \mathrm{GeV}^{*}$ for brious blocks of data. with a typical value of 1.4. This xas substantially xorse than the current state-of-t he-art resolution for similar systems 


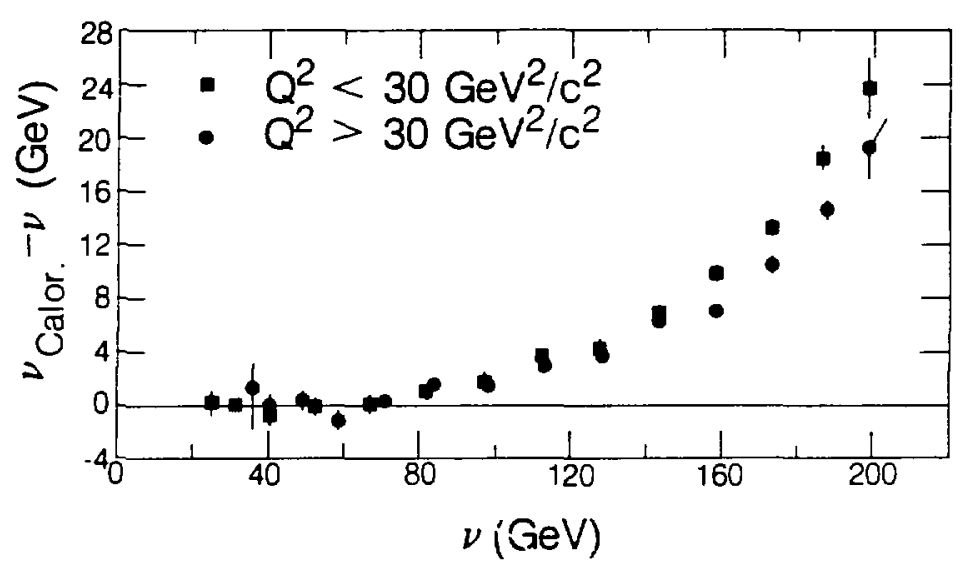

XBL 8310725

Fis. 7.2. $Q^{2}$ dependence in the calorimeter calibration. The larger pulse beight at low $Q^{2}$. high $\nu^{\prime}$ is due to the electromagnetic showers in radiative events. Note: this is a different sample of events than in the calibration example of Fig. T.I.

of $\sigma=1.0 \sqrt{2}$, a walue which we approached in early small-scale tests in a pion beam. The reasons for this degradation are thought to lie primarily in the early calibration stages (counterto-counter and $A D C$ high-to-low), but include also an inherent conponent from fluctuations in the background eaergr loss of the muon (or muons).

The use of the cajorimeter information to improve the energy resolution of the AN1S was described in Sec. 6.4. The relative values of the calorimetric and magnetic resolutions in $\therefore$ Daturally restrict the effect of the calorimeter to low values of $t(5+2)$ ig. 4.11$)$. At large ${ }^{\prime}$. several problems appeared to the calorimetry including the radiative effects mentioned above. ot ber poor calibration bebavior, and lack of agreement between real and simulated calorimeter recolution. For these reasons, we quenched the calorimeter's contribution to $l$, by unweighting the contribution of the calorimeter by a furtber factor of $\left(\mathrm{V} / \mathrm{l}^{\prime} \mathrm{c}\right)^{2}$ for $" \geq \mathrm{ic}_{\mathrm{c}}$. For the 215 (93) Gel. data. "'c was 80 l $401 \mathrm{Gel}$.

\section{Chamber efficiency}

Missing chamber hits can seriously degrade the reconstruction and fitling of tracks. To model correctly the acceptance and resolution of the MDSS, the efficiencies of the proportional 


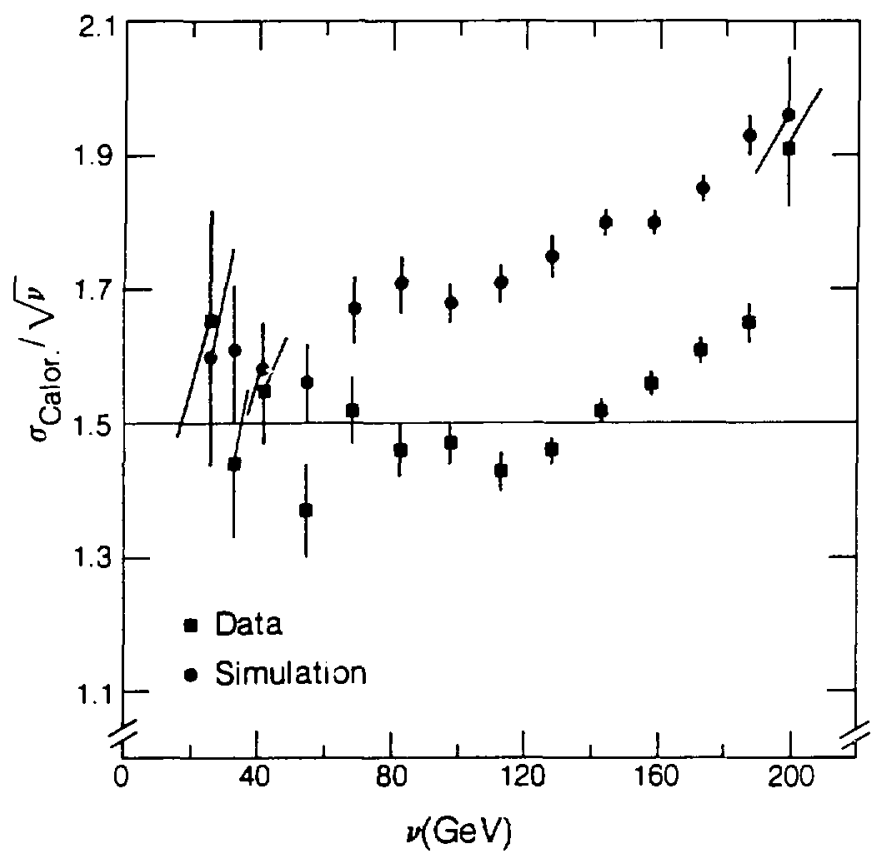

$X B L 8310-728$

Fig. 7.3. Calorimeter resolation. The eircles show the results of the calculation when applied to simulated erents generated with $\sigma_{\mathrm{s} \text { lor }}=1.5 \sqrt{\bar{u}}$. The method thus somexhat overestimates the resolution. The simulated resolution -as later adjusted to match that of the data. 
and drift chambers had to be measured and incorporated into our simulation programs. This was done in a track-oriented fashion by counting the missing chambers on successfully reconstructed tracks. This metbod inadvertant!f includes in the chamber ineficiency track finding deflciences due to other causes. We iteratively corrected for this by using the raw measured inefliciencies in the simulation program, measuring the resulting rate of missing hits on tracks, comparing the result to the rate in real data. and making appropriate adjustments. Each wire plane in each proportional chamber was measured separately. with allowance for correlations within a chamber due to the induced signal read out. In each case, the result was fltted to

$$
\epsilon(x, y)=a-b e^{-r / a} .
$$

where $a, b$, and $s$ are ftted parameters and $r$ is the radial distance from the beam center in that chamber. The fits were perforwed separately for $\mu^{+}$and $\mu^{-}$running because the lower intensity of the $\mu^{-}$beam led to improved elliciency. The average PC efficiencies were quoted in Sec. 4.f. The drift chambers sutfered no loss of eflciency in the beam. A constant efficiency of 0.98 ais used for all drift chambers.

\subsection{Trigger counter efficiency}

The discorery of a large, position-dependent jnefticiency in some of the paddle counters that make up the deep inelastic trigger was a crucial one. The systematic pattern of ineffliency. largest near the beam and decreasing (impioving) toward the top and bottom was almost exactly that needed to create spurious $Q^{2}$ dependence - a disaster for an experiment attempting to measure precisely logaritbmic scaling violations. Fortunately, the existence of parallel subtriggers in the deep inelastic trigger allowed this discovery and also the measurement and mapping of the inefficiency with triggered (i.e., recorded) events. The existence of the inefficiency was verifled by hand-scanning a sample of events suspected to contain missing paddle counters. The possibility that the inefficiency was in the recording of the hits, and not in their contribution to the trigger. was eliminated by the insufficient rate of events with a recorded pattern of trigger counters which failed to satisfy the trigger. Eventually the evidence was incontrovertiblc, as in Fig. 7.4 which shows horizontal scans across pairs of uaddle counters at two locations in the spectrometer. Two common features are visible. In Fig. $T .4 i$ the vertical edge between a good and bad counter is show $\mathrm{n}$. In $\mathrm{b}, \mathrm{a}$ depression at the center of a paddle is obvious. The calbration of this efflciency was of extreme importance and will be discussed in some detail.

The method used to measure the effiency took advantage of the fact that. For a muon penetrating more than three paddles, those paddles outside of any satisfied subtrigger are redundant and can be checked in an eftciency measurement. Thus the events satisfying any subtrigger proride an unbiased sample for the study of the flve other trigger banks. In this discussion. "penetrated" means the muon passed through the counter: "lit" means that the passage was observed by the phototube and amilible to the trigger. L'sing sucb events. most of the active region of the paddles could be mapped. The exceptions were (hose regions which could not be penetrated by a muon that also penetrated tbrce ot ber counters. There were iwo sucb regions: the outer edges (away from the beam) of paddles in the first three trigger banks, and. more importantly. the inside edges of the last three trigger banks. Of these. the most crucial was the inside edge of bank 6 , through which every low $Q^{2}$ event in the experiment fassed. How these regions were fllled in is described below.

Ysing the entire s3mple of 800000 analrzed deep inelastic events, efficiency maps of ea h paddle counter were prepared on a grid with $6 \mathrm{~cm}$ spacing in $I$ and fire cells in $y$ ranging from $3 \mathrm{~cm}$ high near the beam to $18 \mathrm{~cm}$ high at the top and bottom of the spectrometer. Since 


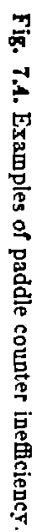

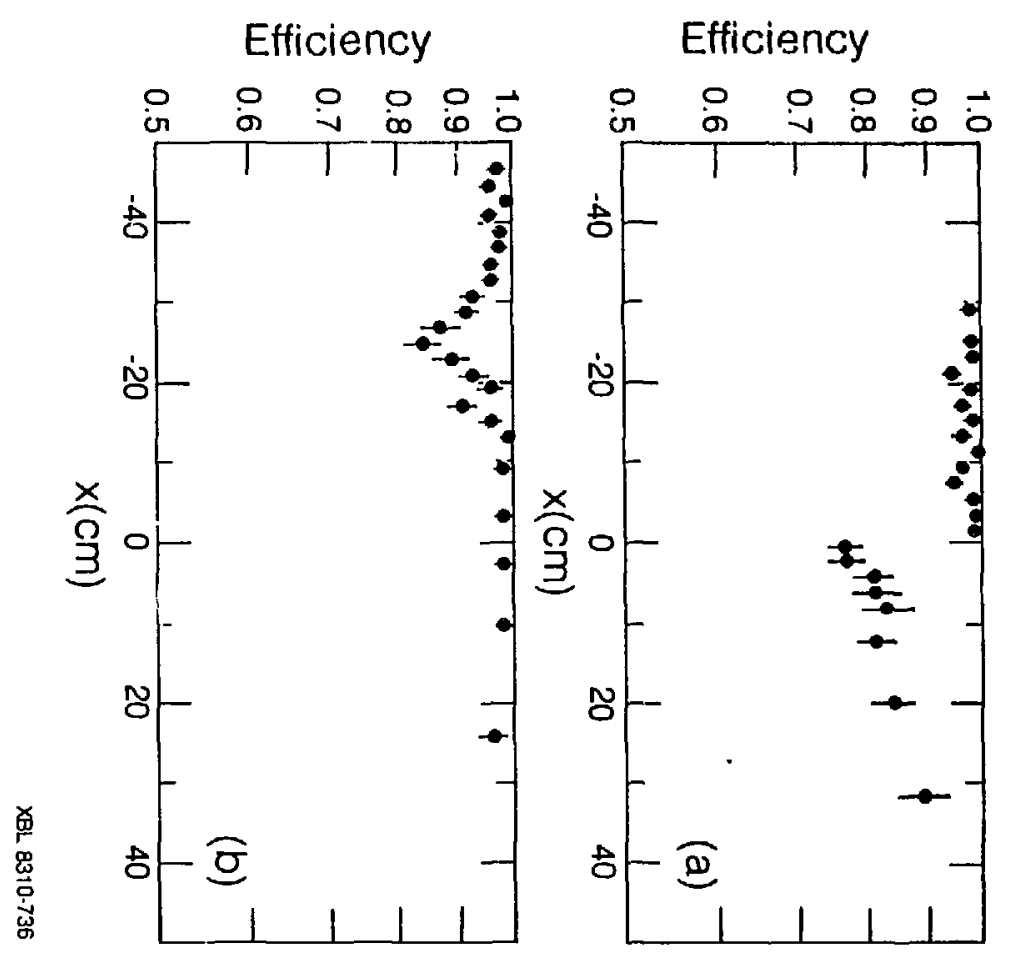


time dependence was obserted in the effiency of sereral counters, the data set was divided into four blocks and four separate maps were produced. Where no time dependence was noticeable. the total sample was used for each map. Much of the time dependence was abrupt, and most orcursed betricen the first block, which was not used in the deep inelastic analysis. and the rest In regions where mapping was possible, the effiency in each cell was typically measured to better than $5 \%$.

Originally, the unreachable regions at the inside edges of the back counters were filled in by linearly extrapolating in $y$. This was checked using a small sample of trimuon triggers that 1riggered in the staves upstream. This test showed no systematic problems tith the extrapolation. Further tests with a large sample of multimuon triggers still shoted no systematic differences between the multimuon and deep inelastic maps in the extrapolation region. but sereral individual cells showed large discrepancies. Although these discrepancies were of random sign, in the MLIS the relationship between geometry and kinematics is such that these did not average out. After this realization. which was the key to the successful analysis of the experiment, each muon from every multimuon event was used to make another set of paddle efficiency maps. Forunately. those multimuon events which reached the paddles did so predominantly in the critical region missing from the deep inelastic map. Before including the multimuon dat a. the two sets of maps were compared in the regions where both measured the efficiency well. This was done to check for a possible bias due to fake tracks in the high background multimuon sample. the fear of which originally prevented their use in the maps. The 180 cells that had multimuon and deep inelastic efficiency measurements with a precision of better than $5 \tau_{c}$ indicated that the multimuon wap was low by $\frac{1}{2} c_{c}$. With this correction, the hypotiesis that the two maps Fere the same yielded a $x^{2}$ of 184 for 179 degrees of freedom. The maps were then averaged, giving complete coverage of the critical regions of the spectrometer to better than $10 \%$ and typically to f $c$. Figure $i .5$ is a contour map of a trigger bank, showing typical good and bad counters. The completed maps were included in the apparatus simulation for the calculation of the acceptance of the MMS.

Knowing the trajectory of a scattered muon, one can calculate the trigger efficiency for that track using the information from th. maps. For muons penetrating more than the required three paddles. the efficiency of course foes up. The statistical uncertainties attached to the penetrated map cells can be similarly combined to give an uncertainty in the trigger efficiency for a single event. The distributions of trigger eficiency and uncertainty for the events used in the deep inelastic analysis are shuwn in Fig. 7.6. The mean efficiency and uncertainty are 0.83 and 0.057 , respectifely, with the effiencies of $94.5 \%$ of the events known to better than $10 \%$. Although counting on averaging to smooth statistical errors in the paddle maps is risky, one can count on at least a four-fold averaging from the four quadrants of the MMS which, alter adding the two magnet polarities are identical except for paddle efficiency. (For the $93 \mathrm{GeF}$ sample. the symmetry is only two-fold because only one MMS polarity was permitted.) Looking at events from indiridual $x-Q^{2}$ bins used in the $F_{2}$ analysis shows that at least five map bins in eacb quadrant of each trigger bank are illuminated in the worst (lowest $Q^{2}$ ) case. Thus, even in the worst case, the effiency measurements for events in a single $I-Q^{2}$ bin are uncorrelated enough to reduce the effects of statistical errors by a factor of four or flve. Allowing for different combinations of map cells in different trigger banks reduces the correlation furt her. The residual uncertainty in our results due to trigger counter effiency will be discussed further in the section on systematic errors. 


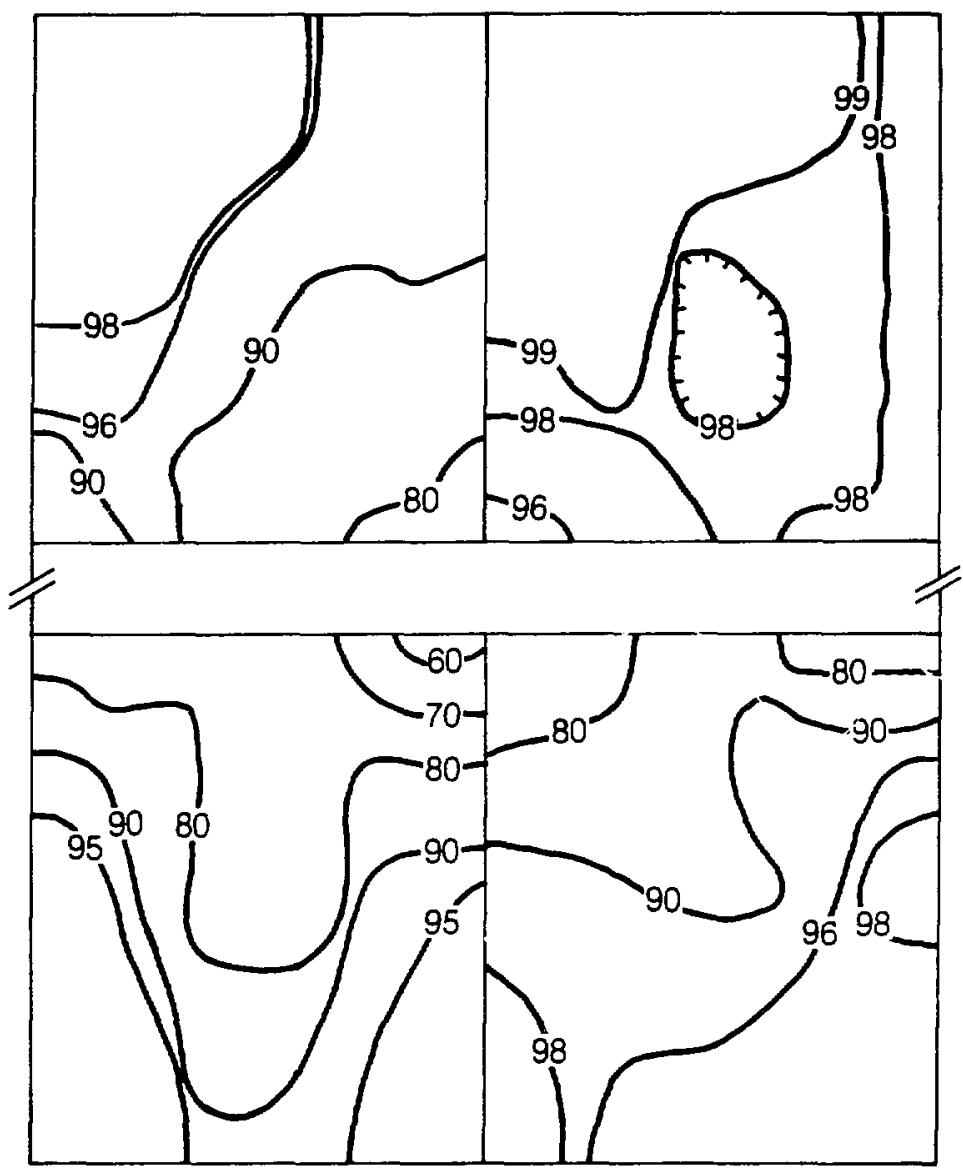

Trigger bank 5 efficiency map (percent)

Fig. 7.5. Efficiency contour map of one trigger hodoscope showing counters of varring quality. 


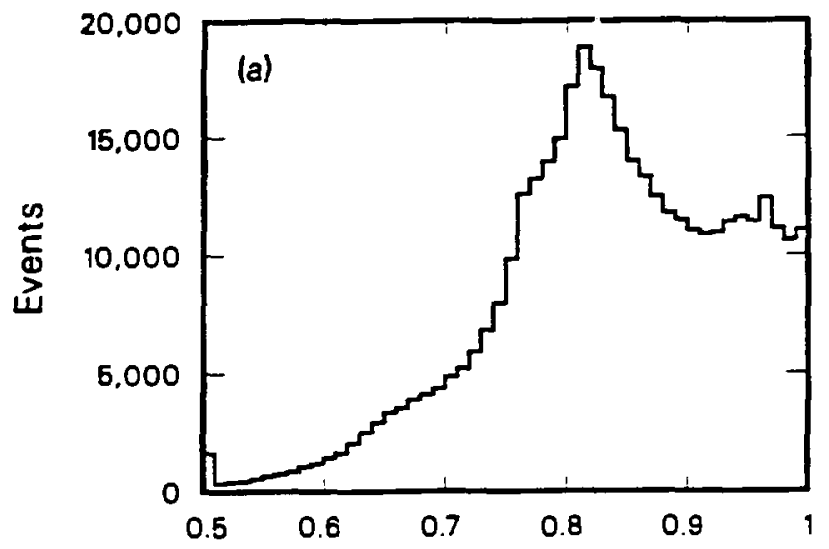

Trigger efficiency

XCG $8311-7289$

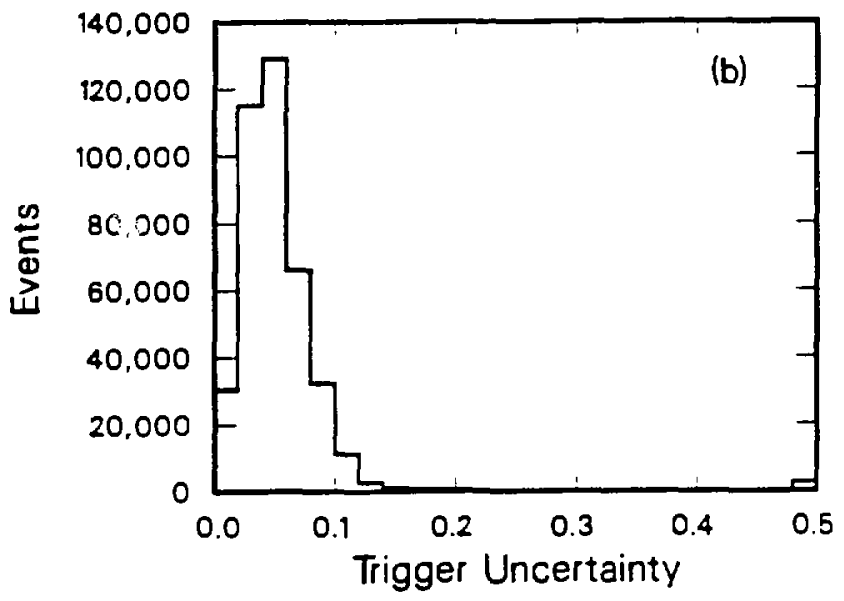

XCG $8311-7290$

Fig. 7.6. Total triggering effleiency (3) and its uncertaints (b) calculated for individual erents in the $215 \mathrm{Gel}^{-}$data. 


\section{Chapter 8 \\ The Apparatus Simulation}

ROS: SerentF-sir - love.

GLIL /musing!: The law of probability, if bas been oddly asserted, is something to do with the propasition that if sir monkeys ... if six monkeys were...

ROS: Game?

GLIL: Were they?

ROS: Are you:

Tom Stoppard, Rosencreots end Gujdenstern afe Dead

\subsection{Acceptance calculation}

After event reconstruction, both the number of incident muons and the number of detected scattered muons as a function of $Q^{2}$ and $r$, or any other set of rariables, are known. From direct measurement of the various componencs of the IDIS, the number of target nucleons is also known. To turn these numbers into a scattering cross section, one further quantity is needed. This is the acceptance of the MMS

$$
A\left(x, Q^{2}\right)=\frac{N^{\text {rig }}\left(x, Q^{2}\right)}{N^{\operatorname{scat}}\left(x, Q^{2}\right)}
$$

which is the fraction of the scattered muons which successfully trigger the apparatus. This is primarily a geometrical quantity depending on what range of angles and energies will project scattered muons into the paddle counters of the trigger banks. It also includes the efficiency of the trigger counters and can be extended to include the eficiency of the reconstruction programs. With these latter quantities measured, a simple apparatus might allow the acceptance to be calculated analy:ically, say as a multiple integral over allowed solid angle and energy. The MLMS does not represent such a case. The integration over the large beam size. distributed target. energ: loss. and multiple scattering, as weil as scattered energy and angle make such an analytic or even numerical integration ditficult. When variables atfecting reconstruction efflency such as PC efficiency, $\delta$-rays, and the splashes from hadronic and eleciromagnetic showers are added to the integrals, the task becomes impossible.

Instead of integrating over the buge phase space defined by all of these variables. we use a Monte Carlo simulation to sample the various regions of this space with frequencies proportional 10 : heir likelihood. To do this, simulated eveats are generated and propagated through a computer 
representation of the MMS. For each process of interest, a random value of the relesant mariable. say an MCS angle in a single steel plate, is chosen at each occurance from a measured or calculated distribution of that rariable. With a large number of generated event $s$. it is hopeci that even the more obscure neighborhoods of the phase space will be sampled. In the deep inclastic simulation. muons were propagated through more than $2 \times 10^{9}$ steel plates.

With the acceptance known, the cross section can be calcuiated from

$$
d \sigma\left(x \cdot Q^{2}\right)=\frac{D\left(x \cdot Q^{2}\right)}{I T A\left(x \cdot Q^{2}\right)}
$$

Where $I$ and $D$ are the numbers of incident and triggered muons and $T$ is the number of target nucleons per wit area. There is a more convenient form of this expression that allows full advantage to be taken of the details of the simulation. Wiriting $A$ as it is calculated in the simulation as

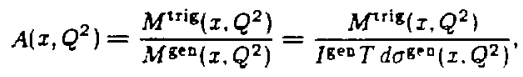

with $M^{\mathrm{gen}}$ and.$^{\text {trig }}$ the numbers of generated and "triggered" events. $I^{\text {ged }}$ the flux of muons represented by the simulated sample, and $d \sigma^{\mathrm{gen}}$ the cross section used to generate the event5, we nrite

$$
d \sigma^{\text {mess }}\left(x, Q^{2}\right)=\frac{\pi D\left(x, Q^{2}\right)}{M\left(I, Q^{2}\right)} d \sigma^{\mathrm{gen}}\left(x, Q^{2}\right) .
$$

In this expression. $d \sigma^{\text {mess }}$ is our measured cross section, $D$ and $M$ are the numbers of triggered dat a and triggered Monte Cario-generated events, and $n$ is the normalization, the ratio of incident muons in the simulated and real samples. Equation 8.4 is the basis of our analysis. Its convenience stems from the fact that no reference is make to untriggered events, either real or simulated. Only triggered simulated events bave to be saved, and they can then be recorded and reconstructed in the same format as data. Witb unit normalization and a perfect apparatus simulation. the interpretation of Eq. 8.4 is simple: $D\left(x, Q^{2}\right)=M\left(x, Q^{2}\right)$ implies $d \sigma^{\text {meas }}=d \sigma^{\text {ren }}$. This equation is also used to make important corrections for resolution smearing. This procedure is described in the next chapter.

It is absolutely crucial that our simulation accurately model the behavior of muons in the MMS in detail. We have no "clean" data sample. Our acceptance and resolution have long tails due to occurances that would be unlikely anywhere but in $5.34 \mathrm{~kg} / \mathrm{cm}^{2}$ of iron. In kinematic regions of small cross section, these tails can dominate the observed population of erents. It is essential that we have confidence in our ability to model not just typical bebavior, but these tails as well.

\subsection{Overview}

Each of the 578 runs used in the analysis was separately simulated. The Monte Carlo simulation of an event proceeds as follows:

- Choose a beam track from the sample of $\mathrm{BV}^{*}$ triggers taken during the data run being simulated.

- Choose the vertex z position randomly in the target.

- Propagate the beam muon from the front of the MMS to the vertes. 
- Use the radiatively corrected deep inelastic cross section to choose the momentum vector of the scattered muon.

- Propagate the scattered track until it leaves the spectrometer, recording the positions in chambers and trigger counters.

- Test the trajectory for triggering.

- If the event triggers, generate the information that appears on a raf data tape: chamber hits, including those from $\delta$-rays and showirs, calorimeter pulse heights, etc.

- Write the event on a magnetic tape in the same format as raw data. with an additional block containing the true (generated) kinematics of the event.

This tape is then read hy the data reduction programs which do not distinguish between real and simulated events except in certain calibration information.

\subsection{Beam and target}

The rapidly rising deep inelastic cross section as $Q^{2} \rightarrow 0$ makes us quite sensitive to the details of the beam phase space. Our trigger makes us especially sensitive to the spatial and angular distributions of the beam in the vertical $(y)$ coordinate. For this reason, and also because frequent adjust ments were made in the beam line settings to improve the yield of muons. We did not try to simulate the beam. We instead used the random selection of beam tracks recorded along witb the triggered events as the parent sample of incident muons for the simulation. For each event, the real beam chamber hits are copied to the simulation output tape and the beam track as reconstructed in position, angle, and momentum at the front of the MLIS is propagated to the interaction vertex by the same routine used for scattered muons (see below). This method. the use of reconstructed values as actual values, supposes that the resolution of the beam system is negligible compared to the width of the beam in all relevant variables. This is calculated to be the case in general, and in Sec. 5.? I discussed the elimination of data where the meeting of 1 his criterion was suspect.

The vertex $z$ position is chosen randomly in the material of the first 13 modules. including piate zero. The scatter may take place in any of the elements of the spectrometer (iron, scintillator, chamher windows, etc.), but the target is always treated as a nucleon in an iron nucleus. $95 c_{C}$ of the scatters take place in the iron plates.

Although the simulation starts with an unbiased sample of the muon beam phase space, the demands of the trigger modify it substantially. Figure 8.1 show 5 the $y$ position distribution of the beam tracks used as input to the simulation, the distribution of simulated events which satisfled the deep inelastic trigger, and the corresponding distribution of real events. The outer edges of the triggered distributions are enhanced by the large low $Q^{2}$ cross section. The 'Iuctuations present in i he dat are due to problems in the beam chambers. Csing the measured distribution as input to the simulation causes the same fuct uations to reappear.

\subsection{Event generation}

When the beam muon reaches the $z$ position of the rertes. its energs (after $d E / d J$ losses 1 is banded to the event generator which fill determine the kinematics of the interaction and the 3-momentum of the scattered muon. The generator uses the deep inelastic cross section 


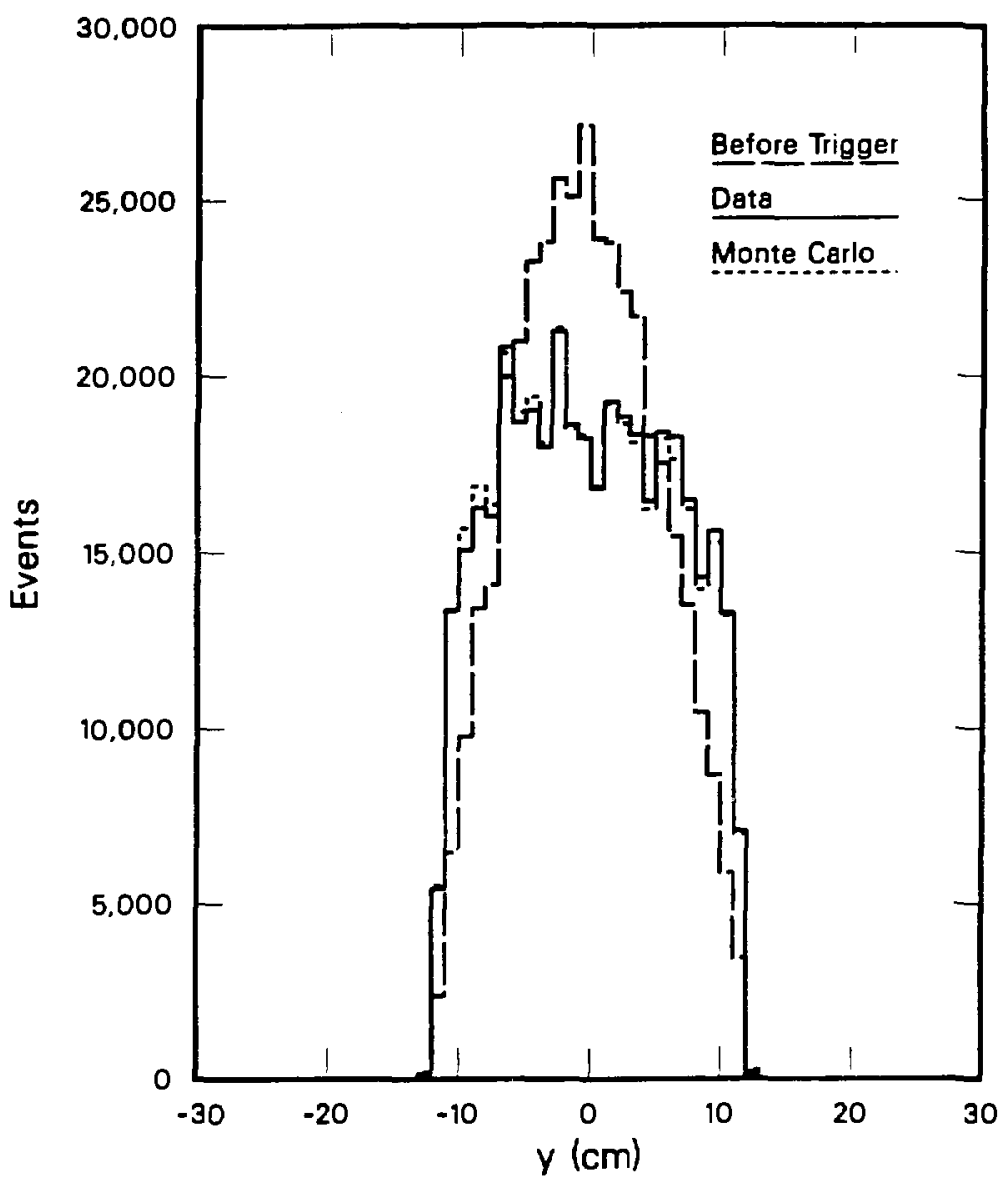

XGC $8311-7283$

Fig. 8.1. Vertical distribution of beam muons entering the MAS, showing the input to the simulation (dotted) and the resulting distribution after triggering (dasbed), normalized to the same total number of events. Also show $n$ is the distribution of real events (solid line). 
in the form (see Eqs. 2.2 and 2.12)

$$
\frac{d^{2} \theta}{d x d y}=\frac{4-x^{2}}{2 M X E} \frac{1}{r^{2} y}\left[1-y+\frac{y^{2}}{2(1+R)}\right] F_{2}\left(s=v / y \cdot Q^{2}=2 M U E_{v}\right) .
$$

It starts by choosing 2 from a $1 / v^{2}$ distribation. Siace this is divergent. xe choose $t$ only above a value $i_{\mathrm{min}}=0.015$. low enough so that the acceptance is nearly zero below $\imath_{\mathrm{m}} \mathrm{n}$. This $\imath_{\mathrm{m}} \mathrm{n}$ corresponds to $Q_{m 2 n}^{2}=5.9(2.5)$ at the average energies at the vertex of the 215 (9.3) $\mathrm{GeV}^{-}$ dat a cets. Vext, $y$ is picked from a $1 / y$ distribution with $y>t$. The remaining terms of the cross section are then calculated, using a nominal beam en ... of $200 \mathrm{GeV}$ and $R=0$. For $F_{2}\left(I, Q^{2}\right)$ we use the parametrization of Buras and Gaemers. ${ }^{51}$ This is a simple analytic form *hich approximates the predictions of QCD by adjusting the $Q^{2}$ dependence to match Eq. 3.13 before fitting the rest of the parametrization to data. The value of $\mathrm{A}$ used is $300 \mathrm{MeV} / \mathrm{c}$. The chosen $(v, y)$ pair is kept or rejected based on a random number comparison with the above cross section. The process is repeated until a valid $(v, y)$ pair is found, at which point the initially chosen $1 / \mathrm{t}^{2} y$ distribution has been shaped to reflect all of Eq. 8.5 .

Once $t$ and $y$ are chosen and the nominal ( $E=200 \mathrm{GeV}$ ) cross section for the event is know, the distribution is shaped once again to take into account the actual energg of the beam muon at the vertex and radiative corrections. Radiative corrections are treated in four parts. corresponding to the diagrams of Fig. 8.2. The first and last diagrams contain the radiation of a real photon in conjunction with the deep inelastic scattering. These are bandled using the peaking approximation which assumes that the radiation leaves the muon direction unchanged and the method of equiralent radiators $w$ hich treats the radiation as a separate energ loss due to a $Q^{2}$ dependent aumber of radiation lengths ${ }^{52}$ A new cross section is calculated for the actual beam energy. including energy loss in the target before the vertes and in the initual equivalent radiator. This cross section is then corrected for the contribution from the vertes and racuum polarization diagrams of Fig. $8.2 \mathrm{~b}^{52}$ To this cross section is added the cross section for the wide angle bremsstrablung (WAB) process of Fig. $8.2 c^{53}$ This can be viewed either as a background process or as a correction to the peaking approsimation - a quasi-elastic scalier wit bost of the deflectun of the muon occuring at the radiative rertes. WAB makes its largest contribution $1 \approx 3 r_{r}$ after cutsl at large $y$ and low $Q^{2}$. Padiative corrections are discussed furt her in Appendis A. The final shaping is done by comparing a random number 10

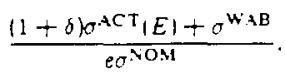

where $\sigma^{A C T}|E|$ is the actual cross section at the iocident muon energy $E$ (including radiarive corrections). 1$]+\delta$ ) is the vertex and racuum polarization correction (see Appendis 1 ). $\sigma^{\text {NoM }}$ is the uncorrected cross section at $200 \mathrm{GeV}$, and $e$ is an enhancement factor. chosen to make this ratio usually less than 1.

For events successfully passing the thal shaping. the outgoing muon's evergy and polar angle with respect to the beam track are

$$
\begin{aligned}
E^{\prime} & =E(1-y \| 1-y) \\
\theta & =2 \arcsin \left[\frac{M x}{2 E(1-y)}\right]^{1 / 2} .
\end{aligned}
$$

wherc $y s$ is the fractional energs loss in the final equinlent radiator. The azimutbal angle $o$ is chored randomly. Eren with the erhancement ractor $e$ in E.q. 8.6. events with a large upstream 


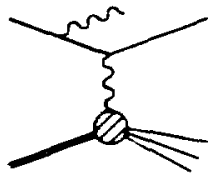

(a)

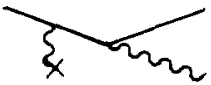

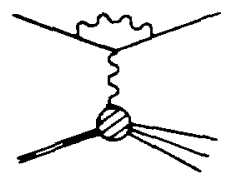

(b)
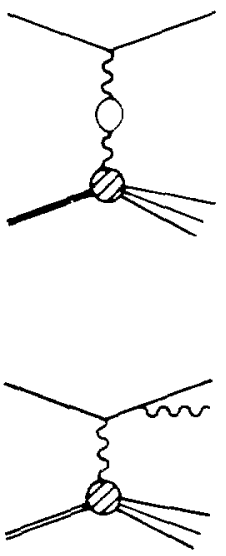

(d)

XBL 8310.716

Fig. 8.2. Radiative corrections. ( $a, d)$ Internal bremsstrahlung: (b) vertes agd vacuun polarization; $(c)$ ride angle bremsstrablung. 
energy loss or a large $\mathbf{W A B}$ contribution will have a shaping ratio greater than one. These events are repeated the appropriate number of times, each time choosing a new $y_{f}$ and 0 . Finally. the outgoing track is rotated from the coordinate system of the beam track to that of the spectrometer and its 3-moment um is handed to the propagation routine.

\subsection{Muon propagation}

The propagation routine constructs the trajectory of the beam muon from the front of the spectrometer to the vertex, and that of the scattered muon from the vertes to where it exits the spectrometer. It projects the path through one element of the MLMS at a time. In active elements (chambers. trigger counters, and calorimeter counters) the position of the trajectory is recorded. In these elements and in the air gaps the muon patb is a straight line. In each iron plate the muon is subjected to energ loss, magnetic deflection, and multiple scattering. Although these processes are treated as occurring only in the iron, the amount of material or $\int \mathrm{B} \cdot d \mathrm{l}$ assigned to each plate includes that in the gaps. It should be noted that both the $d E / d x$ and the $M I C S$ are generated in a form independent of that used to analyze them in the reconstruction programs.

The energy lost by the muon in an iron plate is calculated as the sum of tive terms: average ionization losses (below $0.01 E$ ), stochastically cbosen ionization losses (to knock-on electrons above $0.01 E$ ), average and stochastic losses to pair production (below and above $0.001 E$. respectively), and stochastic losses to bremsstrablung. ${ }^{5-4}$ The probability distribution of energ: losses above $5 \mathrm{GeV}$ for a $200 \mathrm{GeV}$ muon in one of our 4 inch iron plates is show $\mathrm{a}$ in Fig. 8.3. Muons are allowed to lose energy until their range in iron is less than one plate thichness. Their subsequent decay is not simulated. For use in the propagation routine. the energr loss in a plate is divided in half and the balves are applied before and after the deflections due to the magnet and MCS.

The magnetic deflection in each plate is calculated from $t$ he detailed field maps produced during the IAIS calibration. Both the $x$ and $y$ components of the cieflection are included. Each is treated as a single transrerse impulse halfwar through the plate.

Multiple Coulomb scattering $p_{\perp}$ 's are chosen from a distribution calculated using the method of Moliere as illuminated by Betbe. ${ }^{55}$ In four inches of iron. the Rutberford scattering formula predicts a significant probability for scattering at quite large $p_{\perp}$ 's, much larger t ban the $p_{\perp}$ 's anticipated by Molière and Betbe. While the single scattering law used by these gentlemen includes a screening suppression at small $p_{\perp}$, it lacks a bigh $p_{\perp}$ roll-off due to the nuclear form factor. The Molière distribution thus has a tail extending to very large $p_{\perp}$ bich is dominated by single scattering well beyond where the form factor should suppress it (see Fig. 8.4). To cure this. we subtract from the distribution the absolutely normalized Rucherford cross section for single scattering multiplied by $\left(1-\left|F\left(p_{\perp}^{2}\right)\right|^{2}\right)$. The form factor we use is a Gaussian for the nucleus plus an incoherent sum of terms for the protons. The resulting suppression of the tail is also shown in Fig. 8.4. This procedure is of course only approsimate in that it does not deal correctly wit the plural scattering region.

The MCS distribution is important since it determines the moment un resolution of the IMIS. Furthermore. at very low $Q^{2}$ where the cross section is large but the acceptance is small. most of the events that trigger do so wit b the belp of multiple scattering. For this reason. doubling the width of the MCS distribution rould raise our total trigger rate by over $20{ }^{\circ}$. Figure 8.5 shoxs a measure of the MCS that can be observed directly in the data. In the non-bending $|y|$ vien in the MDIS, the fitted position and angle of a track at the vertes are projected in a straight line to the bach of the spectrometer and compared to the MCS-deflected position of the 


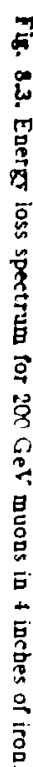

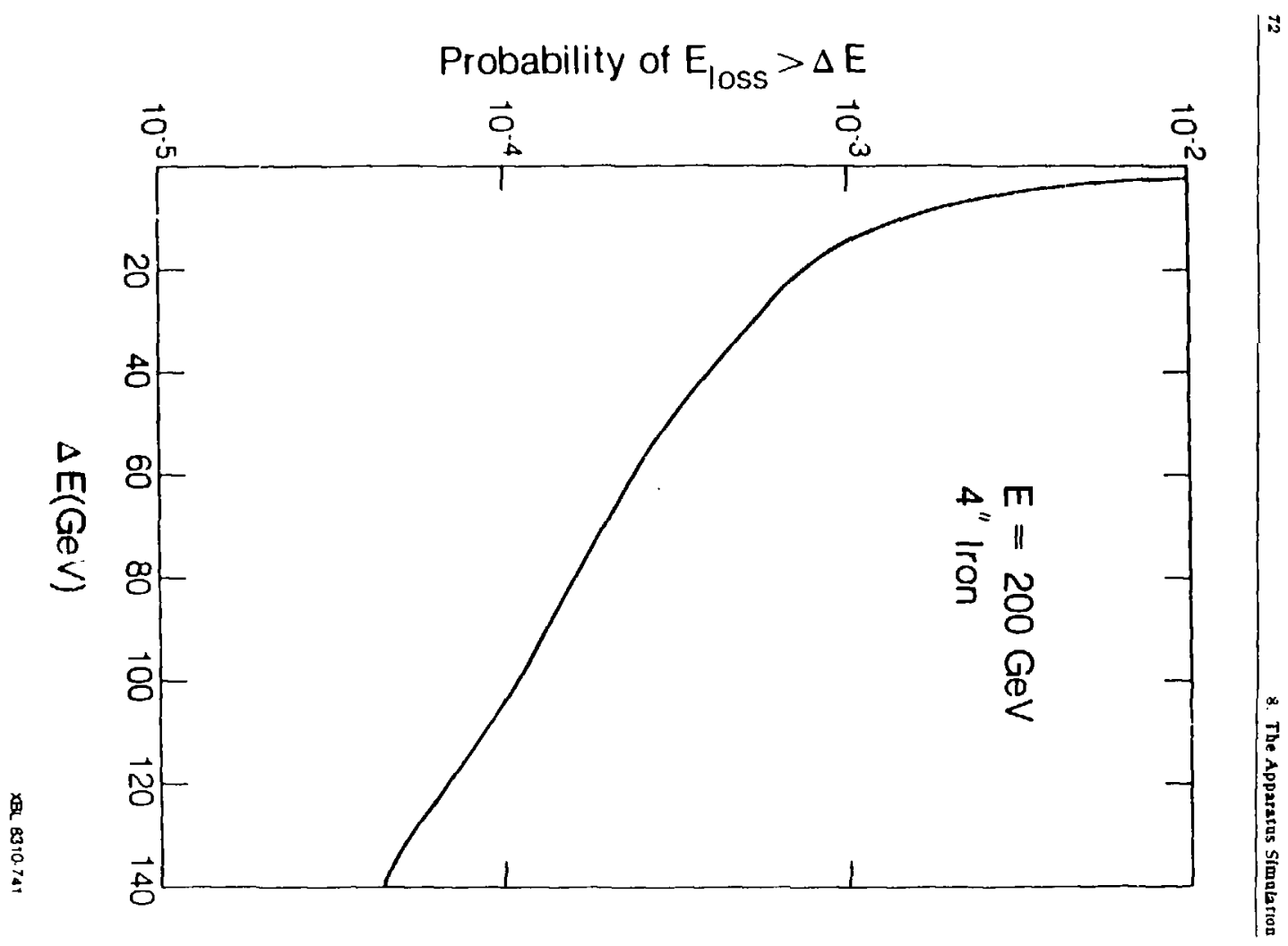




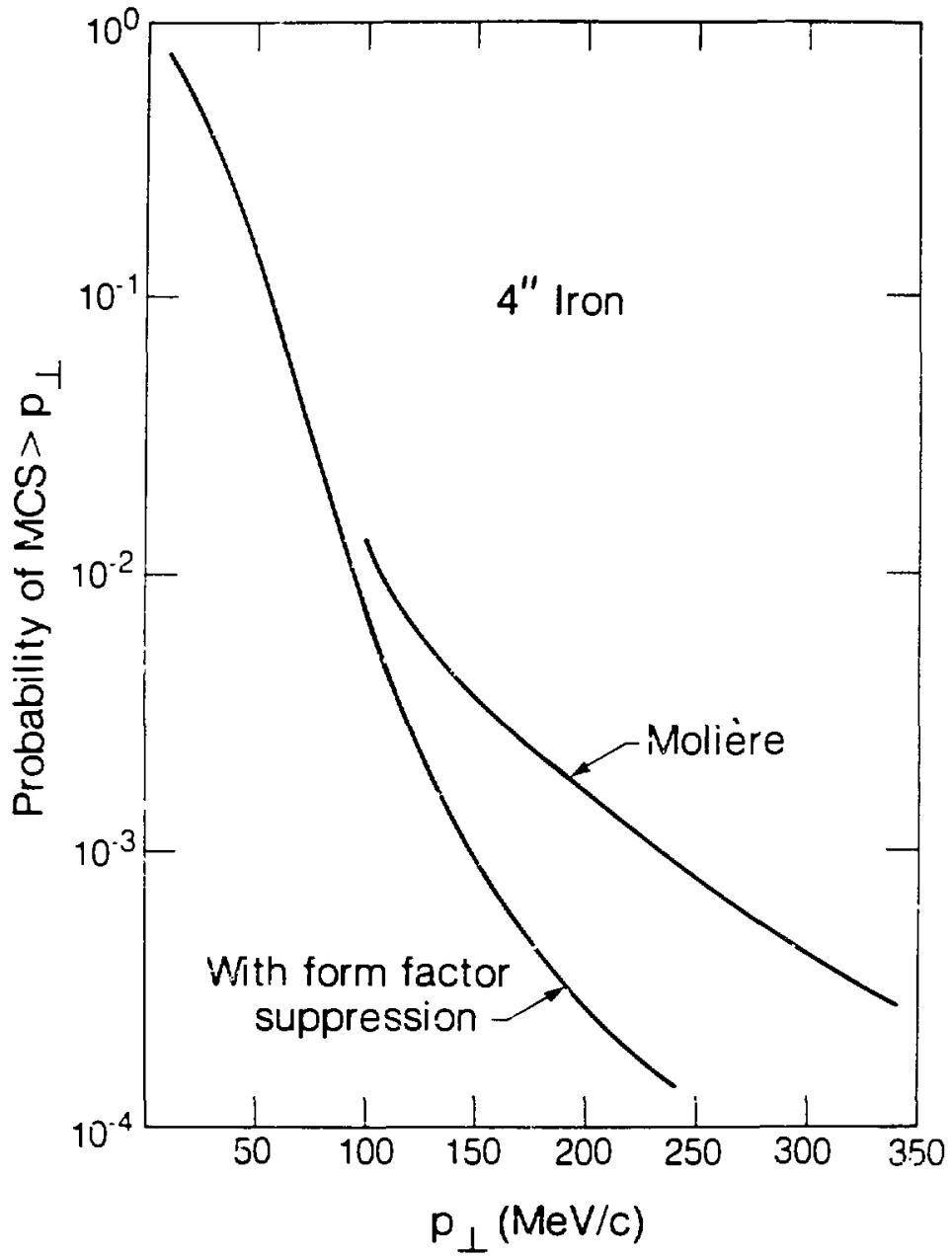

XBL 8310.737

Fic. 8.4. Multiple Coulomb seattering $?_{\perp}$ spectrum for 4 inches of iron. The tall with and witbout form factor suppression of single scattering is shown. 


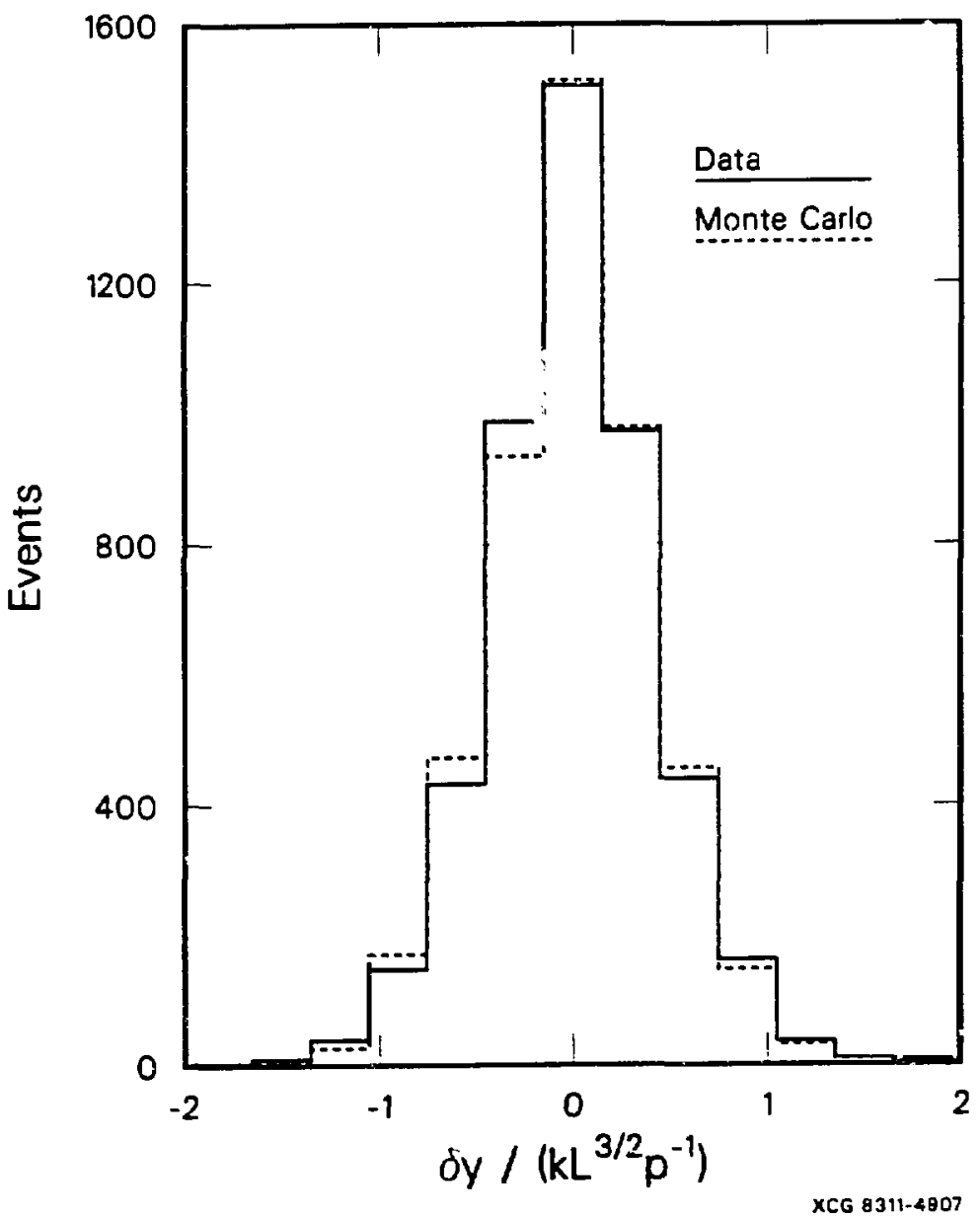

Fig. 8.5. Measured vertical multiple scattering deflection. normalized by the expected value, for real and simulated events. 
track there. To remore patb length and momentum dependence, $\mathrm{h}$ his residual is divided by the expected ralue. proportional to $L \frac{3}{2} / p$ where $L$ is the track length. In the igure, distributions in this rariable are presented for scattered trarks " om our $93 \mathrm{GeV}$ real and simulated data. Although our $y$ position resolution is insufficient to resolve the HCS angles in individual modules land thus the fitted MCS is somewhat suppressed). Monte Carlo study shows that the widtb of this distribution is sencitive to the width of the actual MCS fistribution at the several percent level I he widt bs of the distributions for real and simulated events differ by less than $1.5 c_{c}$.

The propagation of the scattered muon continues unt il the muon leares the spectrometer. The position of the muon in each trigger bank is inspected, and penetrated trigger counters are latched. If the counter is a paddle, the position is used to look up the efficiency in the maps constructed from the data. Only the appropriate fraction of penetrations result in latched counters. The pattern of latched counters is then tested against the deep inelastic trigger requirement.

\subsection{D-ta simulation}

Erents which satisfy the trigger are turned over to a routine which simulates the information produced by the MM!s, including its defects and blemishes. These blemisheschamber hits from showers, missing hits, adjacent wires hit by $\delta$-rays. etc.-are important. As an example, the track-finding failure rate doubles in data taken with an one of the proportional chambers in the middle half of the spectrometer switched off.

The routine begins by turaing the positions of the muon in each chamber into rire and time bin uumbers using the best arailable set of alignment constants. The measured efficiency of each plane is applied, and a history of the experiment is checked to blank out chambers which were switched of during the real run corresponding to that being simulated. Without regard to any specific physical process, positions were jittered before quantization. extra adjacent hits were added. and efficiescies were slightly adjusted to bring into agreement the distributions of $x^{2}$. residuals, and fraction of missing chambers on tracks for real and simulated data. For hits in the induced $\mathrm{PC}$ glanes, it was found that the ratio of double to single hits (ideally unity) was empirically reproduced by applying the measured effciency to each channel of a double hit independently. Cases in which a double hit is changed to a single hit dominate the $x^{2}$ of the $y$ bis because ther are interpreted as offset from their true locations and their weights are increased by a factor of four (see Sec. 6.2). The results of this tuning prores. are shown in Figs. 8.6 and 7. Figure $8.6 \mathrm{a}$ shows distributions of $x^{2}$ per degree of freedom for fits of rea: and simulated tracks in the $r$ and $y$ riews. Figure $8.6 \mathrm{~h}$ shows an overall measure of chamber and track finding effiency, the fraction of hits present on indiridual tracks in the DC's and the $y$ view of the PC's. A malue of 1.0 corresponds to a hit in every chamber penetrated by a track. In Fig. 8.7 we show the idths $(\sigma)$ of the residual distributions $\left(x_{\text {meas }}-x_{\theta t}\right)$ in the drift chambers. where the fit bas been performed ignoring the information in each chamber in turn. These distributions give us confidence that our modeling of the resolution of the MMIS is adequate.

Every deep inelastic event has a hadronic shower which produces a distribution of pulse heights in the calorimeter and also creates a splash of hits in nearby chambers. The response of the calorimeter must be modeled to give dat a and simulation the same resolution at low $\nu$. The splash in the chambers must also be included in the simulation because it affects track finding and vertex resolution. The shower simulation was based on a parametrization of showers from our deep inelastic data. There was no physics input whatsoever.

To arierate the total pulse height in a shorier, the calorimetry simulation used a calibration of $6.0 \mathrm{EP} / \mathrm{GeF}$ and a resolution chosen to match that of the data. The parametrization 


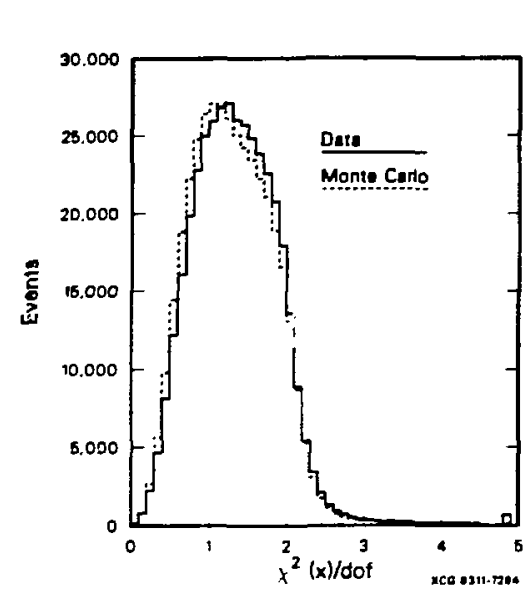

(a)
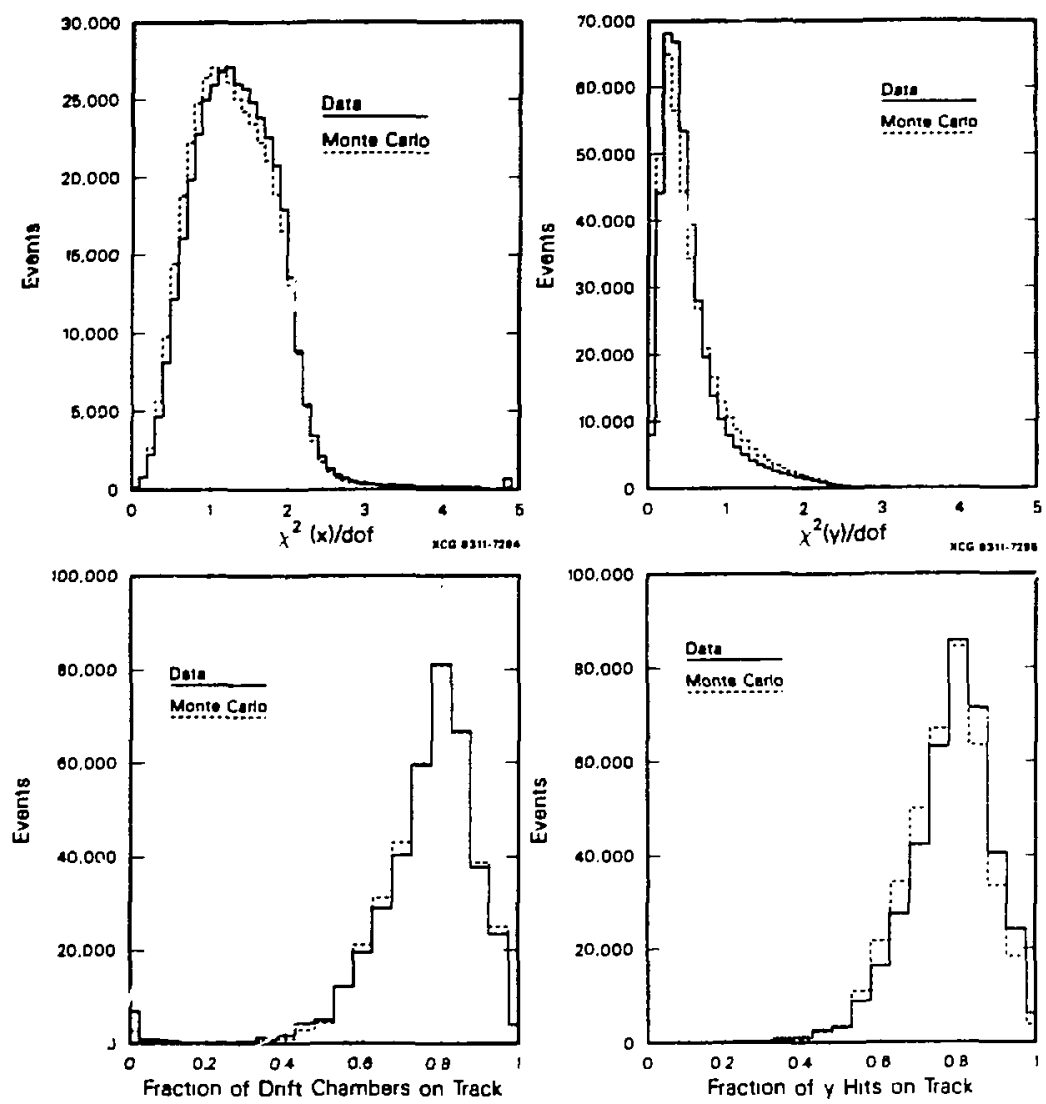

acc esti. rou

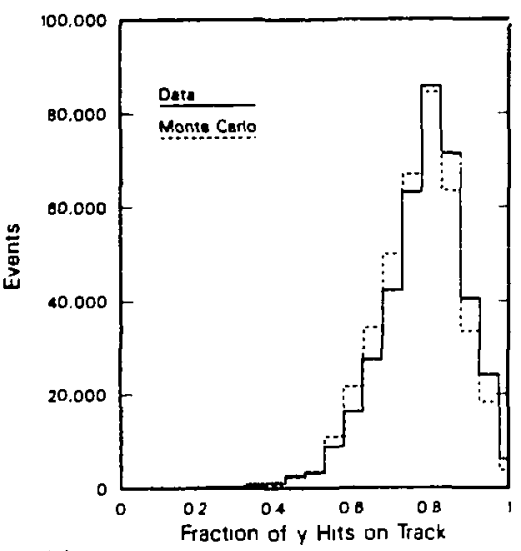

(b)

xct ou

Fig. 8.6. Comparisons of real data and simulation in (a) momentum-ft $x^{2}$ per degree of freedom and (b) fraction of chamber hits on tracks. 


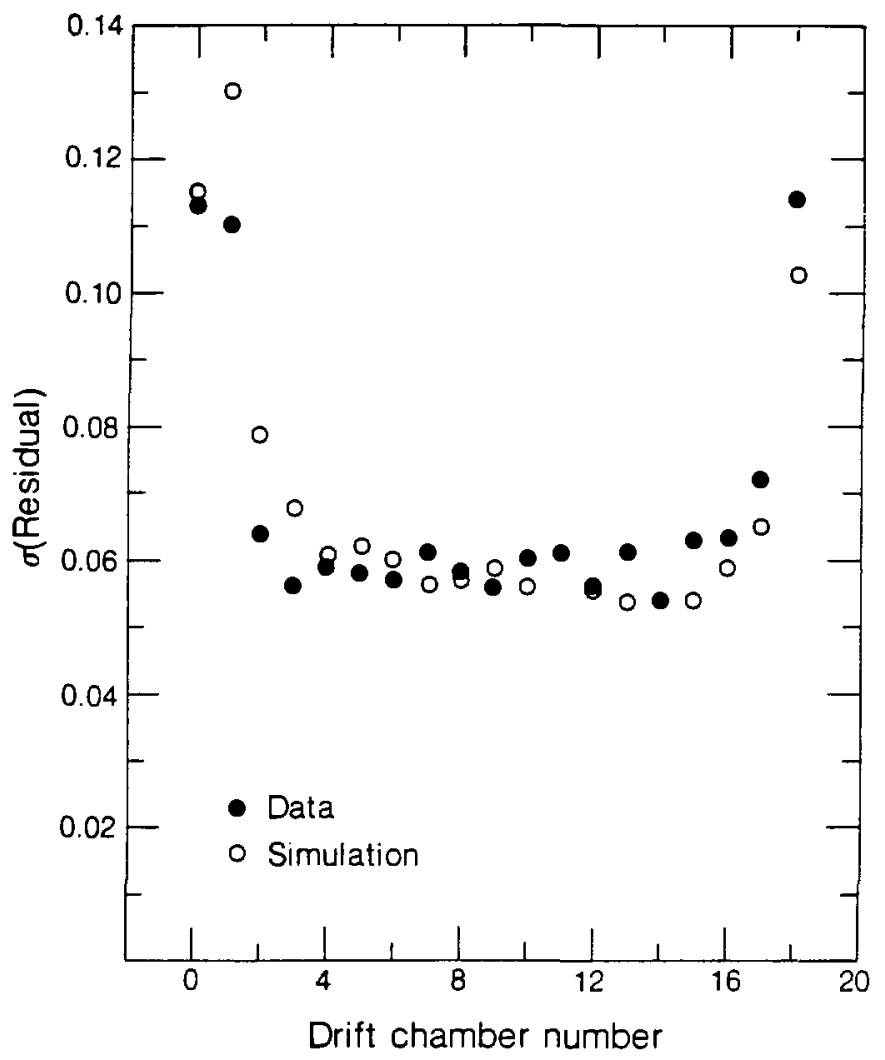

XEL $8310-730$

Fig. 8.7. Widrbs of the residual distributions in each drift chamber. 
of the longit udinal shape began with the determination of the fraction of the energr appearing in each counter following the vertex. This fraction as a function of counter number at 1 wo energies is presented in Fig. 8.8 along witb results from another calorimeter of similar construrt:on ${ }^{45}$ To use tbis information to generate the individual pulse heights sequentially. it $x$ as $c$ ast in the form of fraction of remaining energy per counter. The extremely large fuctuations in these numbers xer. also measured and used in the shower generation. For reconstruction. the F.P-to-GeV calibration of the simulated calorimetry is done with the same procedures used for data. as is the summing of the individual pulse heights to get the shorer energy.

To put hadronic shower splashes into the chambers, the widt h of the splash. the number of hits, and its standard deviation were measured in real showers. The widih was measured as a function of energy and distance from the vertex and was found to be consistent in the DC and various $P C$ planes. Rather than parametrize the number of hits as a function of plane type. energy, and distance from the vertex, a shortcut was employed. The number of hits in a plane was plotted vs the pulse hejght in the adjacent calorimeter counter for each plane type. The result was a family of rapidly rising, then saturating curres, with the level at saturation depending. as expected. on the rire spacing. After calculating the simulated calorimeter counter pulse heights. this relation and its standard jeviation were used to choose a number of hits to put into each chamber plane. These bits were distributed in space as a Gaussian with a width as measured for that energy and distance from the vertex. The center of the shower in earh plane was laid out along a line whose direction was determined by the shower's recoil from the scattered muon.

Electromagnetic showers were parametrized and simulated in a similar fashion. The sample of real showers used to determine the parameters fas found in RV (random) triggers using the calorimeter. The showers showed about $10 \%$ more pulse height per GeV than hadronic showers, with a resolution of $\sigma_{\nu} \approx 2.3 \sqrt{\nu}$. An electromagnetic shower was simulated for each energy loss exceeding $5 \mathrm{GeV}$ in an iron plate. For wide angle bremsstrahlung events, an electromagnetic shower replaced the hadronic shower at the vertex.

\subsection{Operation and performance}

The use of real beam tracks as the beam sample for the simulation prorided an automatic normaiization. both betreen simulated samples and with the data, through the known sampling fraction in the $\mathrm{BV}^{*}$ trigger. The total Monte Carlo simulated data sample corresponds to $1 \times$ the $215 \mathrm{GeV}$ data and $2 \times$ the $93 \mathrm{GeV}$ data. These events were then reconstructed using the same routines used on the real data. Generating and reconstructing the total simulated sample consumed one week of CDC 7600 central processor time.

The remaining flgures in this chapter are evidence of the success with which we can model the complex acceptance and resolution of the MLMS. Figures $8.9 \mathrm{a}$ and $\mathrm{b}$ shor typical real and simulated events. The simulation reproduces all the features of a real event except for hits not related to the beam or seattered muons and the details of low level fuctuations in the single muon pulse heights in the calorimeter. Distributions of real and simulated events in tro rariables that depend only indirecty on the details of the cross secisu are shown in Fig. 8.10. In the figure, the samples are those which result from the application of the analysis cuts and shaping procedure described in the nest chapter. The numbers of events in the two samples have been normalized. Figure $8.10 \mathrm{a}$ is che distribution of events in $\phi$, the azimuthal angle of the scattered muon with respect to the beam track's direction at the vertes. Bending of the beam in the spectrometer before the vertex correlates this coordinate $s$; stem to that of the spectrometer and the distribution has peaks at $\frac{\pi}{2}$ and $\frac{3 \pi}{2}$ corresponding to the vertical scatters required by 


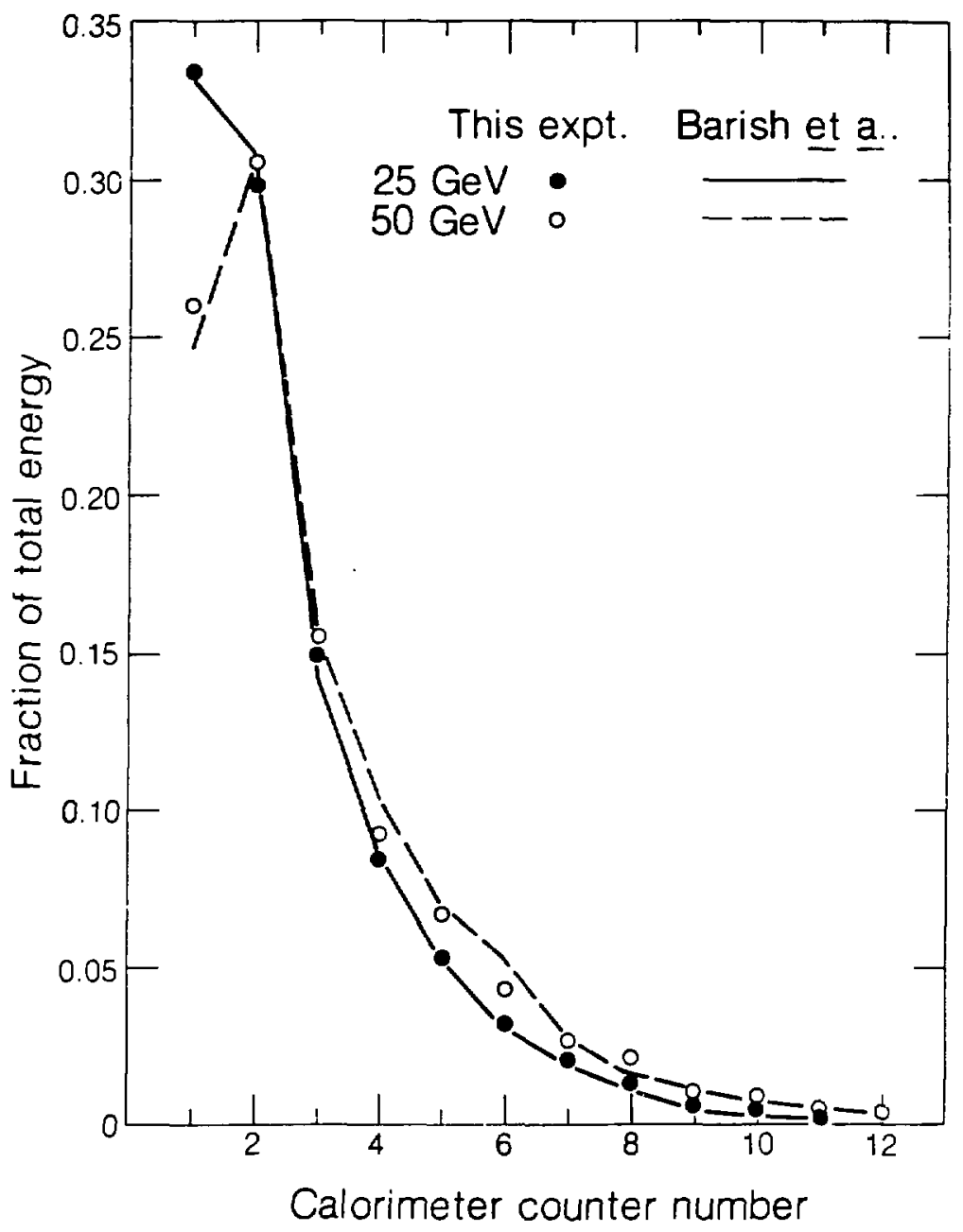

Fig. 8.8. Mean longitudinal distribution of hadronic energy deposition in the calorimeter at $\nu=25$ and $50 \mathrm{GeV}$. At the higher energy, shower maximum is typically after more than 4 inches of iron. The curres show similar measurements from Re? 45. 


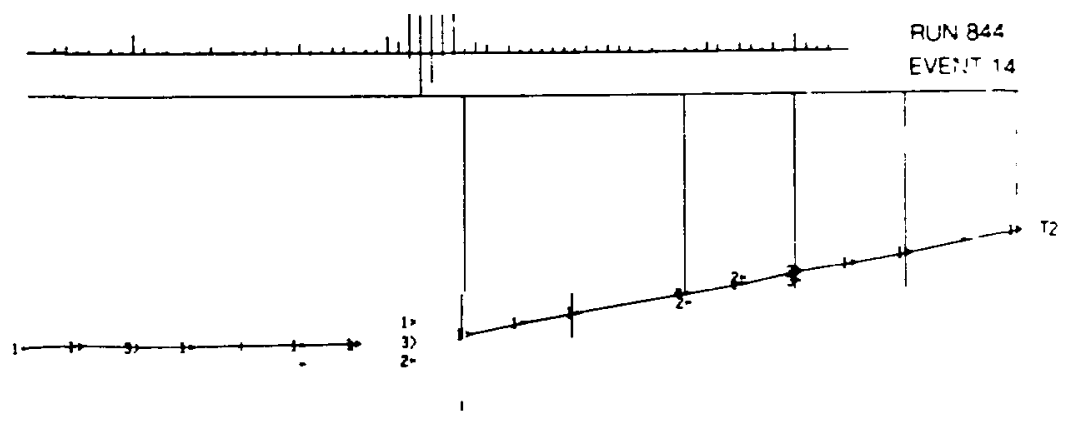

(a) Real

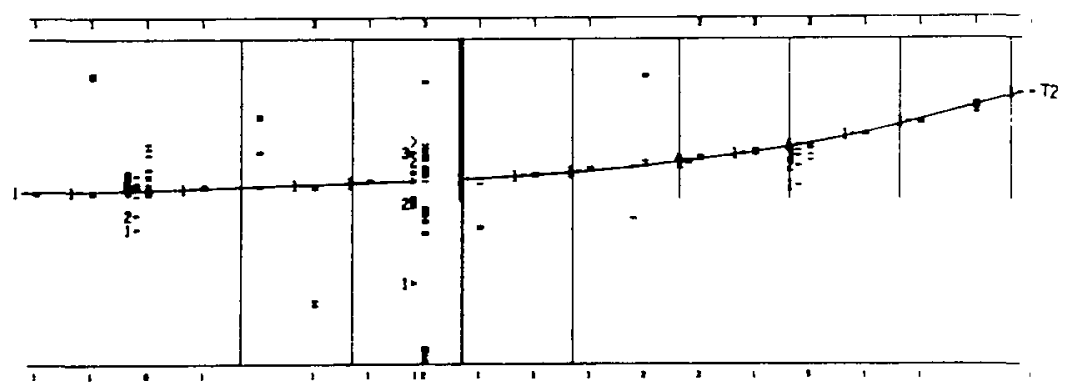

Fig. 8.9. Typical real (a) and simnlated (b) event. Shown are computer displags of raw data from the track flnd:ug program with line segments connecting PC hits assigned to tracks. From top to bottom are: a bar graph of calorimeter pulse heights, low $A D C$ above and high $A D C$ below: the side view of the spectrometer; and the top view. The beam enters from the let. Vertical lines are hit trigger ccunters. In each chamber, the width of the caret indicates the span of consecutive bit wires. In the top view, the upstream and downstream hits in each module are those in the F? and the DC. where both of the left-right ambiguous solutions are 5 hown. Vote the electromagnetic splashes in both the chambers and the calorimeter, and the dense distribution of hits in the badronic shower. 


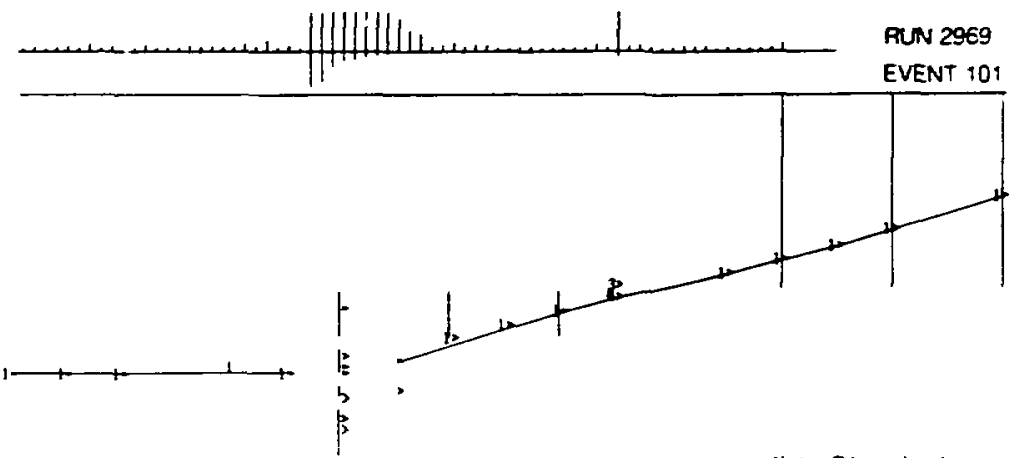

(b) Simulation

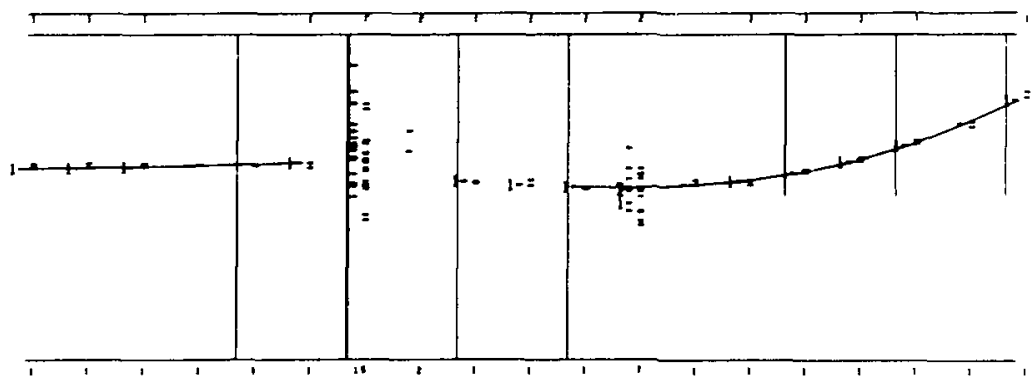

Fig. 8.9. - continued. (b) Typical simulated event. 

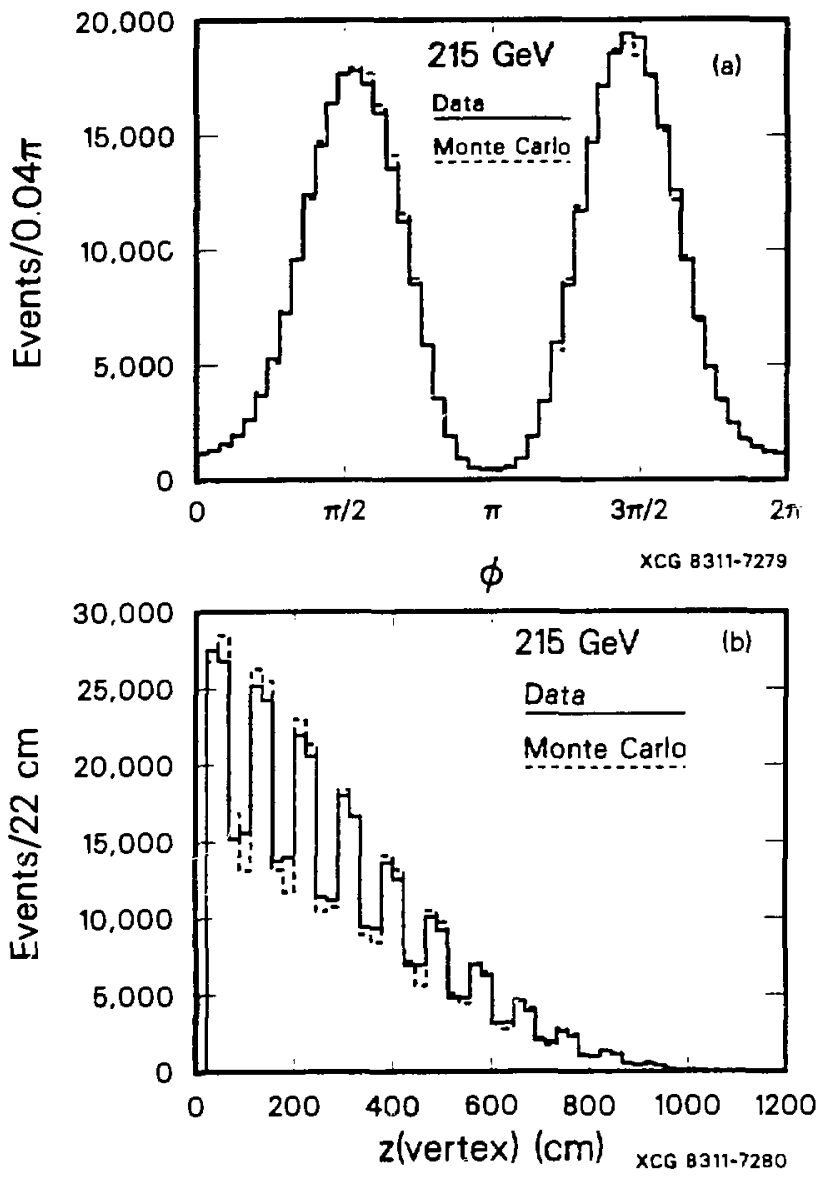

Fig. 8.10. Comparison of real and simulated distributions in (a) azimuthal scattering angle and (b) vertex position along the beam. 
the trigger. Distributions of vertex $z$ locations are shown in Fig. $8.10 \mathrm{~b}$. The mean $=$ positions are $281.0 \pm 0.3 \mathrm{~cm}$ and $280.7 \pm 0.4 \mathrm{~cm}$ for the real and simulated samples. 


\title{
Chapter 9 \\ Analysis
}

\author{
I suid, "No, it's not a case of betng lodely we hape bere - I've been \\ working on this pain tree for eighty-seven years." \\ Niej Young. "The Lest Trip to Tulsa"
}

\subsection{Cuts and the final data sample}

Our analysis is based on the comparison of distritutions of reconstructed real and simulated events with all differences attributed to differences between the aciual cross section and that used in the simulation. In doing this, we are assuming that tbe effects of the apparat us and reconstruction on the distributions are adequately modeled. We apply cuts simultaneously to the real and simulated samples to insure that this is the case.

Our cuts fall into two general categories. The first set of cuts includes those that are necessary because of known limitations in the simulation. The event generator bose events only above $v_{m \text { nn }}=0.015\left(v \equiv Q^{2} / 2 M_{N} \nu\right)$. We must therefore eliminate regions "hich in the data have a contribution from $v<v_{\min }$. We choose a cut of $v>0.025$. The data include events with scattering vertices upstream of the AMS. These are eliminated by a cul in ivertes corresponding to the front of plate 0 . Muons which penetrate a paddle counter near the edge adjoining the staves can veto themselves by producing a $\delta$-ray which hits a stare. While some of the effects of $\delta$-rays on track fluding and resolution were empirically modeled, the effect on the trigger was neglected. For this reason an aperture cut of $0.5 \mathrm{~cm}$ was applied to the inside edges of the paddle counters. A study of real events whose muons passed near the edge of oue paddle and triggered in three other paddles further downstream showed this to be adequate. These cuts remove abont $20 \%$ of the data.

Additional cuts aot absolutely required by the simuiation are applied to remore events that are likely to have been badly misanalyzed or come from regions of poor resolution. We require tisat there be one and only one scattered track and that the reconstructed track be consistent with the recorded pattern of hit cotinters that triggered the event. Because there was no calorimeter counter in the gap following plate 0 . the calorimeter cannot be used to improve t: resn!ution for events with vertices ia plate 0 . We eliminate these events and also, to allow for our 6nite rertex resolution, those in the Brst plate of module 1. Table 9 I shows the set of kinematic cuts applied before analysis. Cuts in parentheses removed ouly an intloitesimal number of events. Tbe regions remored are pcpulated by the extrebe tails of i he resolution and energ. loss distributions. As an example. large missing energr indicates a cati strophic energy loss by 
Table 9-I. Kinematic cuts. Those in parentbeses remove a negligible number of events.

\begin{tabular}{|c|c|c|}
\hline Variable & $93 \mathrm{GeV}^{-}$ & $215 \mathrm{GeV}^{-}$ \\
\hline$E$ & $75<E<96 \mathrm{GeV}$ & $196<E<217 \mathrm{GeV}$ \\
$\nu$ & $>10 \mathrm{GeV}$ & $>20 \mathrm{GeV}$ \\
$E^{\prime}$ & $(>10 \mathrm{GeV})$ & $>10 \mathrm{GeV}$ \\
$W^{2}$ & $\left(>8\left(\mathrm{GeV}^{2} / c^{2}\right)^{2}\right)$ & $>16\left(\mathrm{GeV} / \mathrm{c}^{2}\right)^{2}$ \\
$E_{\text {miss }}$ & $<48 \mathrm{GeV}^{\circ}$ & $<96 \mathrm{GrV}$ \\
\hline
\end{tabular}

the beam muon before scattering. Altbough such losses were included in our simulation, these erents are badly misanalyzed. This second set of cuts removes about $20^{\circ} c$ of the erents surviring the first set. There remain 394522 (39 061) events in the 215 (93) GeV sample.

\subsection{Normalization correction and uncertainty}

The normalization of the data is determined br twe Ased relation between data and simulation achieved through the use of the prescaled sample of real beam trachs in the simulation. Deficiencies in the simulation are revealed by different losses to reconstruction failures and cuts in the real and simulated samples. Tbis necessitates small corrections to the normalization. Before comparing real and simulated event losses, the cumber of rejected real events itself had to be corrected. This is because some rejection categories are dominated by background events which do not appear in the simulation. Thus samples of rejected events from each loss category had to be hand scanned to determine wat fraction were rea! deep inclastic scattering events. This fraction ranged from $0.13 \pm 0.11$ for events rejected due to too large an impact parameter between the beam and scattered tracks to $1.00 \pm 0.08$ for events in which the beam track was badly reconstructed and missed the hit trigger counters. Losses to cuts were also investigated in the same way. Of course, no correction to the normalization was made for the losses due to the "necessary" cuts discussed in the previous section, which were expected to he different for the real and simulated samples. The corrections, accumulated into three major categories, are shown in Table 9-1l. The final corrections to the 215 and $93 \mathrm{GeV}$ samples were $0.020 \pm 0.002$ and $0.028 \pm 0.003$, respectively.

The uncertainty in this correction was but one of the systematic uncertainties in the normalization Others include uncertainties in the target thickness. magnetic feld calibration. beam enerfy, and trigger counter effliencies. Some of these are discussed in more detail in the next chapter. He estimate that in total the normalization uncertainties for the 215 and $93 \mathrm{GeV}$ samples are each $3 \widetilde{r}_{\mathfrak{c}}$. Since some of these uncertainties are common to both samples. the uncertainty in the relative uormalization between the two samples is smaller and is estimated to be $2.5{ }^{c} \mathrm{c}$.

\subsection{Extraction of $F_{2}\left(x, Q^{2}\right)$}

The basis for the exraction of $F_{2}\left(I, Q^{2}\right)$ from the rax population of measured events is Eq. 8.4. Since our doger includes radiative corrections, with as assumption about $R$ we can 
Table 9-I. Normalization correction.

$215 \mathrm{GeV}$ losses

\begin{tabular}{|l|c|c|c|}
\hline Source & Data & Simulation & Correction \\
\hline Track fnding & $0.0015 \pm 0.0007$ & $0.0050 \pm 0.0004$ & $-0.0035 \pm 0.0008$ \\
Erent fitting & $0.0238 \pm 0.0020$ & $0.0148 \pm 0.0003$ & $0.0090 \pm 0.0020$ \\
Cuts & $0.1789 \pm 0.0006$ & $0.1644 \pm 0.0006$ & $0.014+ \pm 0.0008$ \\
\hline Total & & $0.0199 \pm 0.0023$ \\
\hline
\end{tabular}

$93 \mathrm{GeV}$ losses

\begin{tabular}{|l|c|c|c|}
\hline Source & Data & Simulation & \multicolumn{1}{c|}{ Correction } \\
\hline Track foding & $0.0048 \pm 0.0007$ & $0.0080 \pm 0.0003$ & $-0.0032 \pm 0.0008$ \\
Event ftting & $0.0247 \pm 0.0011$ & $0.0059 \pm 0.0002$ & $00188 \pm 0.0011$ \\
Cuts & $0.2089 \pm 0.0018$ & $0.1968 \pm 0.0015$ & $0.0121 \pm 0.0024$ \\
\hline Total & & $0.027 \pm 0.0028$ \\
\hline
\end{tabular}

cancel all the kinematic factors in Eq. 8.4 and are left with an expression for $F_{2}$

$$
F_{2}^{\text {meas }}\left(z, Q^{2}\right)=\frac{n D\left(x, Q^{2}\right)}{M\left(x, Q^{2}\right)} F_{2}^{\text {gea }}\left(x, Q^{2}\right)
$$

The normalization $n$ now includes the corrections of the previous section. Besides providing the acceptance and radiative corrections to the dat3. Eq. 9.1 is also used to correct for resolution smearing effects. The rapidly varying cross section and the poor $x$ resolution at low $v$ (see Figs. 6.4 and 6.5b) make this resolution correction essential. This is illustrated in Fig. 9.1 where the knowledge of the true kinematics of Monte Carlo-generated events is used to show the arerage value of $x_{\text {true }}$ for bins of $x_{\text {measured. }}$. From this flgure one can easily determine where data points will and will not appear in our flasl $F_{2}$ plots. For example. we cannot determine $F_{2}$ at $Q^{2}=128 \mathrm{GeV}^{2} / \mathrm{c}^{2} . x=0.75$, even though we have hundreds of events with (unsmeared) $Q^{2}$ and $I$ in this bin and the acceptance of the MMS in this region is at its masimum. The resolutioninduced feed down from the more populous low $x$ region (see Fig. 6.5b) makes it impossible to isolate a subset of the data at this $Q^{2}$ with an average true $x$ of 0.75 . Even at smaller $x$. there is a discrepanc $y$ between the measured and average true values of $I$ for the same reason.

We correct for this feed dorn by using Eq. 9.1 and information of the type used to make Fig. 9.1. With real and simulated dat a divided into bins of measured $x$ and $Q^{2},\left(I_{m}, Q_{m}^{2}\right)$. the simulated events in each bin are used to determine $\left\langle r_{\text {true }}\right\rangle$ and $\left\langle Q_{1 \text { ryp }}^{2}\right\rangle$ for hat bin. $F_{2}^{\text {gad }}$ is calculated at these average true blues of $I$ and $Q^{2}$ and the resulting $F_{2}^{\text {meas }}$ is assumed 10 refer to these same values. Eq. ?: thus actually reads

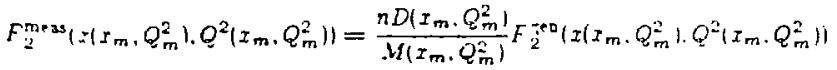

where $I$ and $Q^{2}$ are the averige true miues for the bin of measured rasiables $\left(r_{m}, Q_{m}^{2}\right)$. 


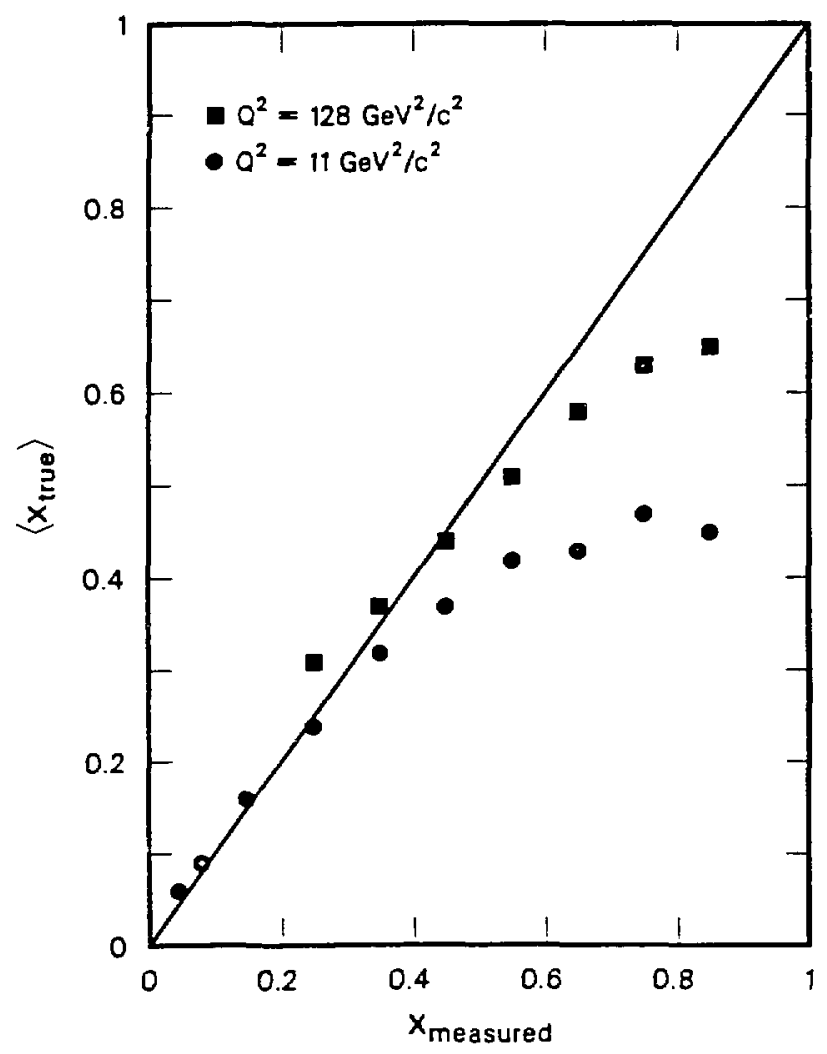

XCG 836-7185

Fiz. 9.1. Limiting effects of $x$ resolution. Plotted is the arerage value of $I_{\text {true }}$ for bins of $z_{\text {messured }}$ as calculated in our apparatus simulation. 
The presence of $F_{2}^{\text {Een }}$ in Eqs. 9.1 and 2 suggests that the $F_{2}^{\text {mes }}$ yielded by this procedure is model dependent. Considering the simulation as merely an acceptance calculation or noting that $M\left(x, Q^{2}\right)$ is proportional to $F 2\left(x, Q^{2}\right)$, demonstrates that, to first order, this is not the case. However, changing the model sufficiently could change the shapes of distributions enough to afect the smearing or the distribution of events within finite sized bins. We remove this model dependence by empirically fitting $F_{2}^{\text {meas }}$ and using this 3 s the $F_{2}^{\text {gen }}$ for a next iteration. This is done by neighting the existing simulated events by the ratio of new to old $F z^{\text {gea }}$. The normalization correction due to the fnal set of cuts must be recalculated for each iteration. The ralues presented above are for the final iteration. The signals that the iteration has converged are l) stability against further change, and 2) identical distributions of data and simulation in many variables. In practice, one iteration satisfes both requirements. In Fig. 9.2 we show comparisoss of dat 3 and Monte Carlo-simulated events in several rariables before and after this iteration for the $215 \mathrm{GeV}^{-}$sample. Only the $I$ and $Q^{2}$ dependence is explicitly changed. Most of the effert in all three variables show $n$ is due to changing the $I$ dependence of $F_{2}$. Note that the shaping is done in real (unsmeared) variables and that agreement in the resolution-dominated tails of measured distributions snch as at large $I$ depends on the accuray of the apparatus simulation. The $93 \mathrm{Gel}^{\circ}$ sample is done separately using the same proceciure.

Other resolution unsmearing techniques were attempted in an effort to extend our measurements further into the high $I$ region. Various global unfolding techniques were tried, ${ }^{50}$ but none were reliable in the regions not covered by the scheme described above. It should be pointed out that the actual unsmearing power of our sueme resides equally in the use of Eq. 9.2 and in the iteration procedure. When the iteration is complete, our parametrization is in fact a determination of $F_{2}$ orer the entire $I$ range, since true valies of $I$ from the entire range are smeared to rield agreement with the measured distribution. Away from the directly measured region. bowever, this determination is limited by the form of the parametrization and is not very sensitive.

Table 9 -III lists our measured ralues of $F_{2}$ in the raw form produced by Eq. 9.2, chat is, *ith each foint referred to its own average true $I$ and $Q^{2}$. The entries have passed a final set of tbree cuts. We have eliminated points whose statistical uncertainty in $F_{Z}$ is greater than $40 \sigma_{\sigma}$. We bare eliminated points from regions with acceptance less than about 0.1 of the masimum. Which corresponds to retaining the region $z>0.06$ and $Q^{2}>15(5) \mathrm{GeV}^{-2} / \mathrm{c}^{2}$ for the $215(93)$ GeV data. We have also eliminated measurements with minimal sensitivity in $I$ by rejecting points for which smearing from other bins contributes greater than $90^{r} \mathrm{c}$ of the events finally appearing in the bin. The correction to $F_{2}$ due to resolution smearing in the retained points is typically $10 \%$, increasing at large $x$. Our results are not sensitive to the esact ralues of these cuts. The interpolation to our final grid of $I$ and $Q^{2}$ is described in the nest chapter. 


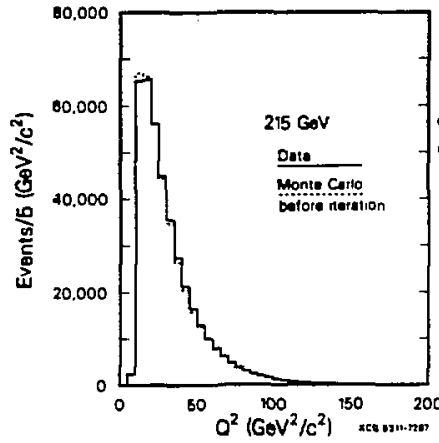

(a)

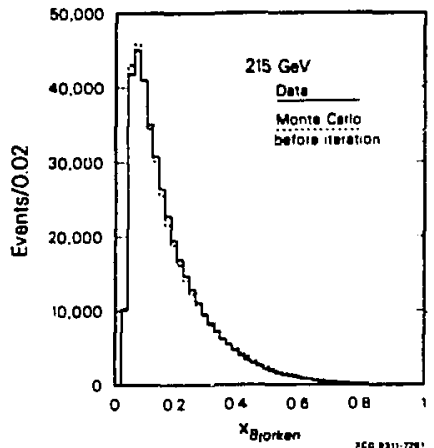

(b)
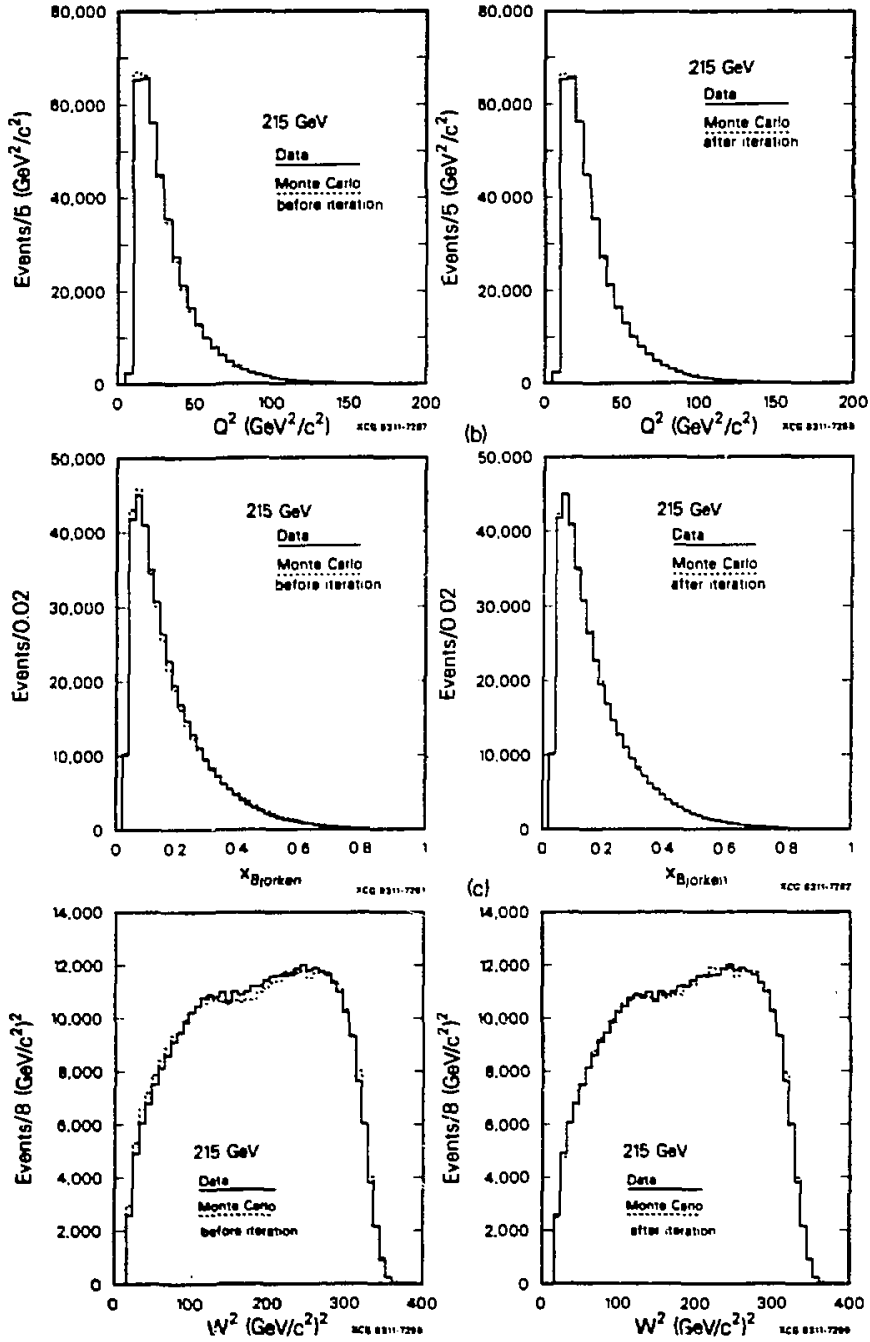

Fig. 9.2. Distributions of real and simulated events before and after the $F F^{\text {en }}$ iteration procedure described in the test. 
Table 9 III Raw $F_{2}\left(x, Q^{2}\right)$ at $93 \mathrm{GeV}$. For each measured bin, $F_{2}\left(x, Q^{2}\right)$ is presented at the average true $I$ and $Q^{2}$ of that bin. Errors are statistical.

\begin{tabular}{|c|c|c|}
\hline$x$ & $Q^{2}\left(\mathrm{GeV}^{-2} / c^{2}\right)$ & $F_{2}\left(x, Q^{2}\right)$ \\
\hline 0.0965 & 5.30 & $0.3890 \pm 0.0105$ \\
0.0992 & 7.13 & $0.3766 \pm 0.0099$ \\
0.1050 & 9.53 & $0.3661 \pm 0.0114$ \\
0.1161 & 12.36 & $0.3729 \pm 0.0228$ \\
0.1591 & 5.29 & $0.3375 \pm 0.0092$ \\
0.1584 & 7.26 & $0.3600 \pm 0.0084$ \\
0.1575 & 9.78 & $0.3478 \pm 0.0074$ \\
0.1601 & 13.30 & $0.3227 \pm 0.0077$ \\
0.1703 & 17.67 & $0.3207 \pm 0.0104$ \\
0.1904 & 22.25 & $0.2716 \pm 0.0201$ \\
0.2343 & 5.33 & $0.2923 \pm 0.0111$ \\
0.2384 & 7.15 & $0.2867 \pm 0.0088$ \\
0.2401 & 9.74 & $0.2666 \pm 0.0075$ \\
0.2398 & 13.28 & $0.2574 \pm 0.0073$ \\
0.2403 & 17.94 & $0.2578 \pm 0.3080$ \\
0.2435 & 24.22 & $0.2436 \pm 0.0091$ \\
0.2647 & 31.06 & $0.2259 \pm 0.0163$ \\
0.28012 & 5.69 & $0.267 i \pm 0.0344$ \\
0.3067 & 7.16 & $0.2257 \pm 0.0103$ \\
0.3131 & 9.62 & $0.2144 \pm 0.0083$ \\
0.3189 & 13.13 & $0.2021 \pm 0.0081$ \\
$0.328-1$ & 17.94 & $0.1986 \pm 0.0083$ \\
\hline
\end{tabular}

\begin{tabular}{|c|c|c|}
\hline$x$ & $Q^{2}\left(\mathrm{GeV}^{2} / \tau^{2}\right)$ & $F_{2}\left(x, Q^{2}\right)$ \\
\hline 0.3299 & 24.19 & $0.1722 \pm 0.0082$ \\
0.3355 & 32.99 & $0.1601 \pm 0.0098$ \\
0.3542 & 42.67 & $01187 \pm 0.0168$ \\
0.3554 & 7.60 & $0.2337 \pm 0.0294$ \\
0.3719 & 9.49 & $0.177-0.0093$ \\
0.3825 & 12.81 & $0.1602=0.0089$ \\
0.3946 & 17.83 & $0.1495 \pm 0.0085$ \\
0.4049 & 24.11 & $0.1374 \pm 0088$ \\
0.4162 & 32.33 & $0.1169 \pm 0090$ \\
0.4199 & 42.87 & $0.0964 \pm 0.0112$ \\
0.4101 & 9.87 & $2.2540 \pm 0.0132$ \\
0.4269 & 12.61 & $0.1 ! 41 \pm 0.0085$ \\
0.4551 & 17.40 & $0.1092 \pm 0.0: 90$ \\
0.4646 & 23.62 & $0.0911 \pm 0.00$ \\
0.4768 & 32.27 & $0.0764 \pm 0.00$ \\
0.4951 & 43.11 & $0.0542 \pm 0.002$ \\
0.5146 & 56.97 & $0.0499 \pm 0.0176$ \\
0.4871 & 17.09 & $0.0888 \pm 0.0096$ \\
0.5091 & 23.22 & $0.0830 \pm 0.0105$ \\
0.5235 & 31.05 & $0.0550 \pm 0.0090$ \\
0.5332 & 42.23 & $0.0467 \pm 0.0093$ \\
0.5801 & 56.65 & $0.0444 \pm 0.0138$ \\
\hline & &
\end{tabular}


Table 9-III. -continued. Raw $F_{2}\left(x, Q^{2}\right)$ at $215 \mathrm{GeV}$. For eacb measured bip. $F_{2}\left(x, Q^{2}\right)$ is presented at the average true $I$ and $Q^{2}$ of that bin. Errors are statistical.

\begin{tabular}{|c|c|c|}
\hline$x$ & $Q^{2}\left(\mathrm{GeY}^{-2} / c^{2}\right)$ & $F_{2}\left(x, Q^{2}\right)$ \\
\hline 0.0736 & 16.31 & $0.3852 \pm 0.0053$ \\
0.0781 & 18.13 & $0.396 i \pm 0.0652$ \\
0.0925 & 18.29 & $0.3794 \pm 0.0028$ \\
0.1026 & 23.70 & $0.3667 \pm 0.0028$ \\
0.1175 & 30.61 & $0.3603 \pm 0.0045$ \\
0.1326 & 34.22 & $0.2972 \pm 0.0306$ \\
0.1563 & 18.58 & $0.3261 \pm 0.0037$ \\
0.1564 & 25.06 & $0.3246 \pm 0.0029$ \\
0.1575 & 33.56 & $0.325 i \pm 0.0027$ \\
0.1686 & 43.68 & $0.3146 \pm 0.0030$ \\
0.1917 & 55.67 & $0.2765 \pm 0.0055$ \\
0.2438 & 18.68 & $0.2466 \pm 0.0044$ \\
0.2439 & 25.25 & $0.2428 \pm 0.0034$ \\
$0.2+! 5$ & 33.85 & $0.2469 \pm 0.0030$ \\
0.2427 & 45.74 & $0.2446 \pm 0.0029$ \\
0.2470 & 61.64 & $0.2336 \pm 0.0030$ \\
0.2670 & 78.30 & $0.2113 \pm 0.0045$ \\
0.2922 & 92.56 & $0.2120 \pm 0.0410$ \\
0.3220 & 18.45 & $0.1940 \pm 0.0052$ \\
$0.3 \pm 55$ & 24.91 & $0.1852 \pm 0.0040$ \\
0.3306 & 33.82 & $0.1830 \pm 0.0034$ \\
0.3307 & 45.67 & $0.1762 \pm 0.0031$ \\
0.3352 & 61.68 & $0.1644 \pm 0.0030$ \\
0.3421 & 83.72 & $0.1589 \pm 0.0033$ \\
0.3598 & 106.28 & $0.1427 \pm 0.0054$ \\
0.3780 & 18.58 & $0.1514 \pm 0.0063$ \\
\hline & & \\
\hline
\end{tabular}

\begin{tabular}{|c|c|c|}
\hline$x$ & $Q^{2}\left(\mathrm{GeY}^{-2} / c^{2}\right)$ & $F_{2}\left(x Q^{2}\right)$ \\
\hline 0.3920 & 24.60 & $0.1416 \pm 0.0045$ \\
0.4049 & 33.48 & $0.1319 \pm 0.0036$ \\
0.4091 & 45.32 & $0.1276 \pm 0.0033$ \\
0.4149 & 61.01 & $0.1086 \pm 0.0028$ \\
0.4163 & 82.30 & $0.1073 \pm 0.0032$ \\
0.4303 & 111.10 & $0.0927 \pm 0.0035$ \\
0.4431 & 136.49 & $0.0788 \pm 0.0095$ \\
0.4175 & 19.48 & $0.1282 \pm 0.0129$ \\
0.4313 & 24.12 & $0.1188 \pm 0.0054$ \\
0.4514 & 33.04 & $0.1047 \pm 0.0041$ \\
0.4684 & 44.44 & $0.0919 \pm 0.0035$ \\
0.4855 & 60.45 & $0.0720 \pm 0.0028$ \\
0.4822 & 80.8 .1 & $0.0661 \pm 0.0027$ \\
0.4933 & 109.42 & $0.0672 \pm 0.0035$ \\
0.5148 & 143.17 & $0.0407 \pm 0.0037$ \\
0.4815 & 32.60 & $0.0892 \pm 0.0052$ \\
0.5159 & 44.07 & $0.0670 \pm 0.0037$ \\
0.5255 & 59.27 & $0.0566 \pm 0.0033$ \\
0.5353 & 79.92 & $0.0492 \pm 00030$ \\
0.5575 & 107.94 & $0.0394 \pm 0.0029$ \\
0.5812 & 142.99 & $0.0382 \pm 0.0040$ \\
0.6056 & 186.69 & $0.0394 \pm 0.0161$ \\
0.5880 & 79.71 & $0.0393 \pm 0.0036$ \\
0.6187 & 107.23 & $0.0296 \pm 0.003 i$ \\
0.6026 & 137.44 & $0.0285 \pm 0.0042$ \\
0.6511 & 182.23 & $0.0120 \pm 0.0043$ \\
\hline & & \\
\hline
\end{tabular}


Chapter 10

Results

It's time to ast or cot buit ... or both.

T. Markiewicz (apoctyphal)

$10.1 F_{2}\left(2, Q^{2}\right)$

The raw $F_{2}$ measurements of Table 9 -II are interpolated onto a grid of flxed true $I$ and $Q^{2}$ using the final ft (see nes section) as an interpolating function. The grid is chosen to be the same as that used to bin the eveats initially in measured $I$ and $Q^{2}$. In $Q^{2}$, the effects of resolution smearing; and thus of the interpolation, are minor. This is aided by the approximate scaling of $F_{2}$. In $\tau$. $\left\{x_{\text {true }}\right\}$ may bear little resemblance to the measured $\tau$ bin it corresponds to. For this reason, the $F_{2}$ measurements were interpolated to the bin center of the bin in the tnal grid which contained ( $x_{\text {true }}$ ) for that point, independent of which bin of measured $z$ rhe point came from. Thus a point from a bin of measured $x$ between 0.6 and 0.7 with $\left(z_{\text {true }}\right)=0.42$ would be interpolated to $I_{\text {t: }}=0.45$. This procedure can result in several bins producing measurements at the same true $I$ and $Q^{2}$. A minimutn requirement sn our extraction method is that these measurements give the same vaiue of $F_{2}$, that is, that they have the same ratio of real to simulated events. Figure $I 0.1$ shows the $x=0.45$ section of our $215 \mathrm{GeV}$ data. The agreement between points at the same $Q^{2}$ (originally from different bins of measured $x$ ) is good. These points are aver'aged after fitting to create our final $F_{2}\left(I, Q^{2}\right)$ results.

The resulting measurements of $F_{2}\left(x, Q^{2}\right)$ are presented separately for the two beam energies in Table 10-I and in Fig. 10.2. We have assumed $R=0$ and have made no correction for Fermi motion. Tùe $F_{2}$ values thus represent $F_{2}^{\mathrm{Fe}} / 56$. The effects of Fermi motion and non-zero $R$ are discussed below. As a consequence of the above procedure, the measurements refer to $F_{2}$ at the indicated true $I$ and $Q^{2}$ and are not bin averages. Because of this fact. a fex of the points appear to have $y>1$. Tbis is simply because the center of the $Q^{2}$ bin lies above the kinematic limit and the original $\left(Q_{\text {true }}^{2}\right)$ was near the lower edge of the bin. In using these measurements, these points caI be ignored if the $y$ dependence is important, or the raw measurements of Table 9-III can be used. The listed and plotted errors are statistical. As systematic errors cause correlated shifts in the dat a we do not attach systematic uncertainties to individual points - systematic errors are discussed in detail in a later section. One powerful test of the internal consistency of our dat $\mathrm{a}$ is the agreement of the 93 and $215 \mathrm{GeV}$ measurements. Figure 10.2 shows this agreement to be quite good. Although the $x^{2}$ tor the orerlap is 33 for 15 degrees of freedom (dof). orer half of this comes from two points. When these are ignored, the $x^{2}$ drops to $14 / 13$ dof. In these 


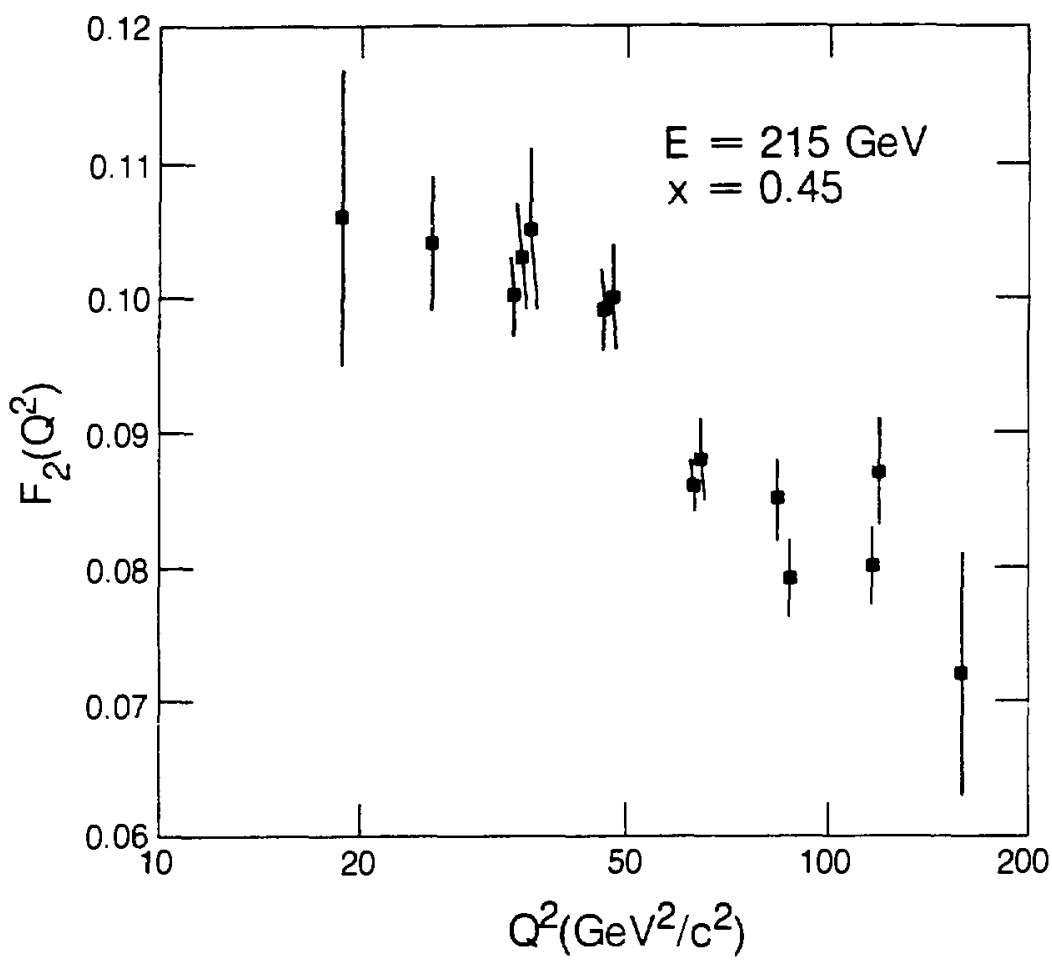

$X B L$ L 8310.722

Fis. 10.1. The $x=0.45$ bin of our $215 \mathrm{GeV} F_{2}\left(x, Q^{2}\right)$ measurement, after interpolation to the grid of true $x$ and $Q^{2}$, but prior to areraging of measurements assigned to the same point. Values at the same point have been displaced slightly in $Q^{2}$ for clarity. 
Table 10-I. The structure fanction $F_{2}\left(x, Q^{2}\right)$ on a fixed grid of $x$ and $Q^{2}$. $R=0$ is assumed and no Fermi motion correction has been applied. (Table continued on next page.)

\begin{tabular}{|c|c|c|c|c|}
\hline$x$ & $Q^{2}$ & $F_{2}(93 \mathrm{GeV})$ & $F_{2}(215 \mathrm{GeV})$ & $F_{2}($ combiged $)$ \\
\hline \multirow[t]{5}{*}{0.08} & 5.54 & $0.3997 \pm 0.0108$ & \multirow{5}{*}{$\begin{array}{l}0.3894 \pm 0.0026 \\
0.3906 \pm 0.0030\end{array}$} & $0.3997 \pm 0.0108$ \\
\hline & 7.52 & $0.3905 \pm 0.0103$ & & $0.3905 \pm 0.0103$ \\
\hline & 10.2 & $0.3855 \pm 0.0120$ & & $0.3855 \pm 0.0120$ \\
\hline & 18.8 & & & $0.3894 \pm 0.0026$ \\
\hline & 25.6 & & & $0.3906 \pm 0.0030$ \\
\hline \multirow[t]{9}{*}{0.15} & 5.54 & $0.3430 \pm 0.0094$ & \multirow{9}{*}{$\begin{array}{l}0.3309 \pm 0.0037 \\
0.3296 \pm 0.0030 \\
0.3330 \pm 0.0023 \\
0.3303 \pm 0.0031 \\
0.3148 \pm 0.0062 \\
\end{array}$} & $0.3430 \pm 0.0094$ \\
\hline & 7.52 & $0.3657 \pm 0.0085$ & & $0.3657 \pm 0.0085$ \\
\hline & 10.2 & $0.3531 \pm 0.0075$ & & $0.3531 \pm 0.0075$ \\
\hline & 13.9 & $0.3321 \pm 0.0074$ & & $0.3321 \pm 0.0074$ \\
\hline & 18.8 & $0.3364 \pm 0.0109$ & & $0.3315 \pm 0.0035$ \\
\hline & 25.6 & $0.3017 \pm 0.0223$ & & $0.3291 \pm 0.0030$ \\
\hline & 34.9 & & & $0.3330 \pm 0.0023$ \\
\hline & 47.2 & & & $0.3303 \pm 0.0031$ \\
\hline & 64.1 & & & $0.3148 \pm 0.0062$ \\
\hline \multirow[t]{11}{*}{0.25} & 5.54 & $0.2811 \pm 0.0102$ & \multirow{11}{*}{$\begin{array}{l}0.2413 \pm 0.0043 \\
0.2373 \pm 0.0033 \\
0.2386 \pm 0.0029 \\
0.2370 \pm 0.0029 \\
0.2300 \pm 0.0029 \\
0.2253 \pm 0.0048 \\
0.2479 \pm 0.0479\end{array}$} & $0.2811 \pm 0.0102$ \\
\hline & 7.52 & $0.2764 \pm 0.0085$ & & $0.2764 \pm 0.0085$ \\
\hline & 10.2 & $0.2576 \pm 0.0072$ & & $0.2576 \pm 0.0072$ \\
\hline & 13.9 & $0.2480 \pm 0.0070$ & & $0.2480 \pm 0.0070$ \\
\hline & 18.8 & $0.2481 \pm 0.0077$ & & $0.2429 \pm 0.0038$ \\
\hline & 25.6 & $0.2365 \pm 0.0088$ & & $0.2372 \pm 0.0031$ \\
\hline & 34.8 & $0.2351 \pm 0.0170$ & & $0.2385 \pm 0.0029$ \\
\hline & 47.2 & & & $0.2370 \pm 0.0029$ \\
\hline & 64.1 & & & $0.2300 \pm 0.0029$ \\
\hline & 87.1 & & & $0.2253 \pm 0.0048$ \\
\hline & 118.3 & & & $0.2479 \pm 0.0479$ \\
\hline \multirow[t]{10}{*}{0.35} & 7.52 & $0.1961 \pm 0.0084$ & \multirow{10}{*}{$\begin{array}{l}0.1729 \pm 0.0039 \\
0.1686 \pm 0.0030 \\
0.1692 \pm 0.0031 \\
0.1628 \pm 0.0029 \\
0.1545 \pm 0.0028 \\
0.1534 \pm 0.0032 \\
0.1475 \pm 0.0056\end{array}$} & $0.1961 \pm 0.0084$ \\
\hline & 10.2 & $0.1874 \pm 0.0059$ & & $0.1874 \pm 0.0059$ \\
\hline & 13.9 & $0.1791 \pm 0.0058$ & & $0.1791 \pm 0.0058$ \\
\hline & 18.8 & $0.1808 \pm 0.0061$ & & $0.1752 \pm 0.0033$ \\
\hline & 25.6 & $0.1578 \pm 0.0076$ & & $0.1671 \pm 0.0028$ \\
\hline & 34.8 & $0.1500 \pm 0.0091$ & & $0.1672 \pm 0.0029$ \\
\hline & 47.2 & $0.1192 \pm 0.0168$ & & $0.1615 \pm 0.0029$ \\
\hline & 64.1 & & & $0.1545 \pm 0.0028$ \\
\hline & $8 \% .1$ & & & $0.1534 \pm 0.0032$ \\
\hline & 118.3 & & & $0.1475 \pm 0.0056$ \\
\hline
\end{tabular}


Table 10-I. - continued.

\begin{tabular}{|c|c|c|c|c|}
\hline$z$ & $Q^{2}$ & $F_{2}(93 \mathrm{GeV})$ & $F_{2}(215 \mathrm{GeV})$ & $F_{2}$ (combined) \\
\hline 0.45 & 10.2 & $0.1009 \pm 0.0107$ & & $0.1009 \pm 0.0107$ \\
& 13.9 & $0.0989 \pm 0.0074$ & & $0.0989 \pm 0.0074$ \\
& 18.8 & $0.1092 \pm 0.0071$ & $0.1082 \pm 0.0109$ & $0.1089 \pm 0.0059$ \\
& 25.6 & $0.1032 \pm 0.0055$ & $0.1062 \pm 0.0048$ & $0.1049 \pm 0.0036$ \\
& 34.8 & $0.0929 \pm 0.0059$ & $0.1035 \pm 0.0022$ & $0.1022 \pm 0.0021$ \\
& 47.2 & $0.0756 \pm 0.0070$ & $0.1009 \pm 0.0022$ & $0.0986 \pm 0.0021$ \\
& 64.1 & & $0.0882 \pm 0.0019$ & $0.0882 \pm 0.0019$ \\
& $8 \bar{i} .1$ & & $0.0844 \pm 0.0020$ & $0.0844 \pm 0.0020$ \\
& 118.3 & & $0.0833 \pm 0.0025$ & $0.0833 \pm 0.0025$ \\
& 160.7 & & $0.0739 \pm 0.0089$ & $0.0739 \pm 0.0089$ \\
\hline \multirow{2}{*}{0.55} & 25.6 & $0.0611 \pm 0.0077$ & & $0.0611 \pm 0.0077$ \\
& 34.8 & $0.0444 \pm 0.0072$ & & $0.0444 \pm 0.0072$ \\
& 47.2 & $0.0403 \pm 0.0080$ & $0.0522 \pm 0.0028$ & $0.0509 \pm 0.0026$ \\
& 64.1 & $0.0443 \pm 0.0105$ & $0.0469 \pm 0.0027$ & $0.0467 \pm 0.0026$ \\
& 87.1 & & $0.0457 \pm 0.0023$ & $0.0457 \pm 0.0023$ \\
& 118.3 & & $0.0412 \pm 0.0030$ & $0.0412 \pm 0.0030$ \\
& 160.7 & & $0.0353 \pm 0.0025$ & $0.0353 \pm 0.0025$ \\
\hline 0.65 & 118.3 & & $0.0210 \pm 0.0022$ & $0.0210 \pm 0.0022$ \\
& 160.7 & & $0.0171 \pm 0.0025$ & $0.0171 \pm 0.0025$ \\
& 218.3 & & $0.0137 \pm 0.0039$ & $0.0137 \pm 0.0039$ \\
\hline
\end{tabular}




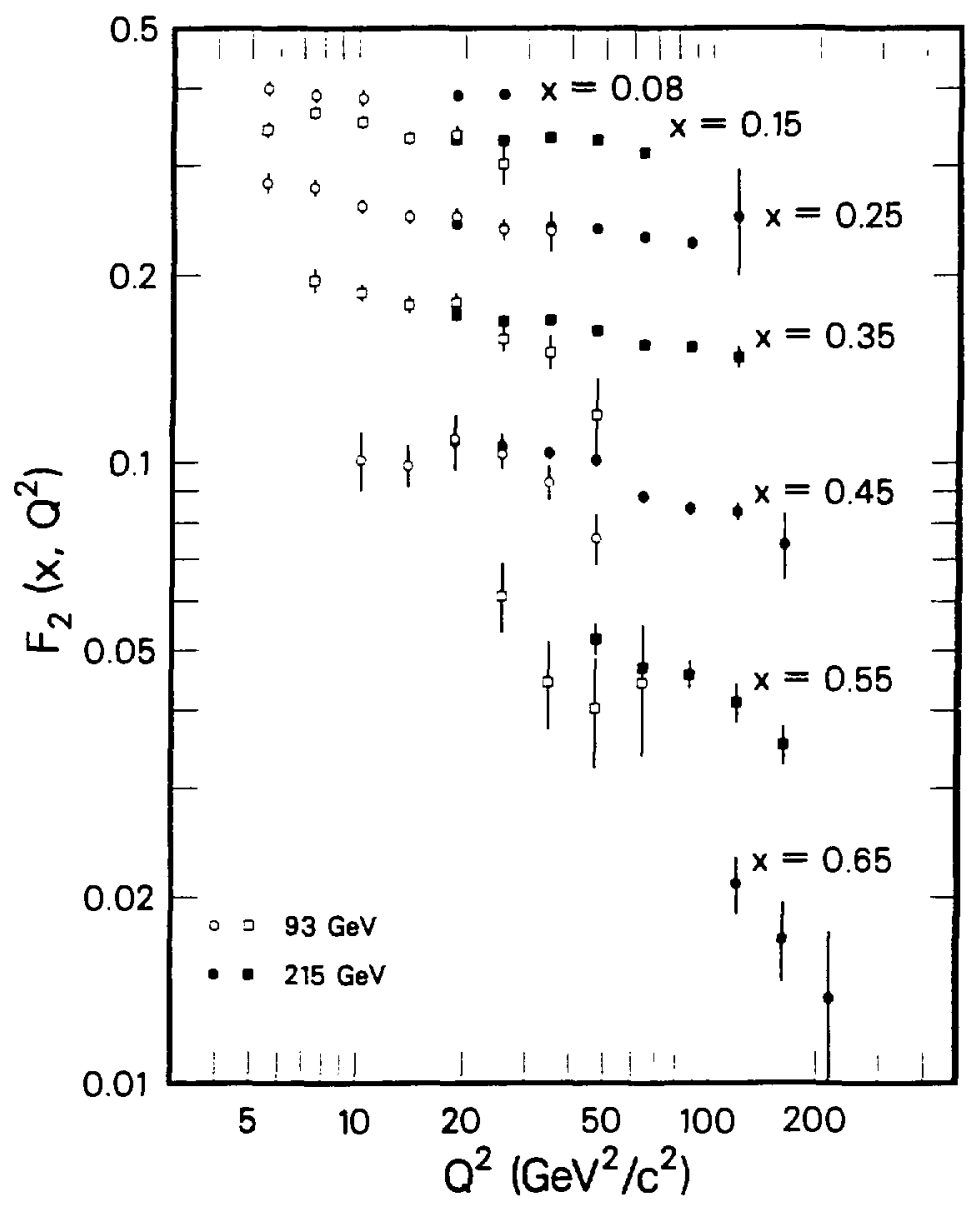

XCG $8311-4902$

Fis. 10.2. $F_{2}$ for nucleons in iron as a punction of $Q^{2}$ at various fixed values of x. 93 and $215 \mathrm{GeV}$ data are shown separately. Errors are statistical. 
data the scaling violation is plainiy visible at $x>0.2$. To treat this quantitatively, we fit the measurements using lorest order quantum chromodynamics.

\subsection{Comparison to lowest order QCD}

Fits are performed to the data of Table 9 -III prior to the interpolation and coalescence of points in $x$. For convenience in fitting, the more gentle interpolation to $Q^{2}$ bin centers is performed before fitting. The core of our fitting program was modifled from a routine hindly provided by R. M. Baruett. Its operation and use are described in Ref. 57 , and summarized here.

The fitting program uses the Atarelli-Parisi method ${ }^{27}$ to calculate the $Q^{2}$ dependence of $F_{2}$ at fixed I predicted by lowest order $Q C D$ for the faror singlet case. Starting with parametrizations of $F_{2}(\tau)$ and $G(x)$ at a reference value of $Q^{2} \equiv Q_{0}^{2}$ and an initial estimate of the QCD scale parameter $\Lambda$, the rcutine numerically integrates the set of simultaneous differential equations of Eq. 3.14 from $Q_{0}^{2}$ to each $Q^{2}$ at which there are $F_{2}$ measurements. There is one singlet-quatk aud one gluon equation for each bin of $x$ with bin center $x_{3}$. At each $Q^{2}$, the predictions for $F_{2}\left(x_{1}, Q^{2}\right)$ are interpolated to the average true $x$ of each measured point at that $Q^{2}$ using a cubic spline. The predicted and measured values are compared and the minimization program MINUTT ${ }^{58}$ is used to vary $A$ and the $Q_{0}^{2}$ parametrization so as to minimize the $\gamma^{2}$ Nir:e that operationally $Q_{0}^{2}$ is largely symbolic, as the data at all $Q^{2}$ are used to determine all the parameters.

Our parametrization at $Q_{0}^{2}=5.535 \mathrm{GeV}^{2} / \mathrm{c}^{2}$ is

$$
\begin{aligned}
& F_{2}\left(x, Q_{0}^{2}\right)=\frac{5}{18} \sum_{s=1}^{2 N_{f}} x f_{2}(x)=A x^{\alpha}(1-x)^{3}(1+\alpha x)+B(1-x)^{7} . \\
& G\left(x, Q_{0}^{2}\right)=C(1-x)^{k} .
\end{aligned}
$$

The two terms of the $F_{2}$ parametrization are inspired by the conceptual division of the quarks into "ralence" and "sea" distributions. The $\frac{5}{5}$ is the average charge squared of the $S($ (2) singlet (valencel or $S[$ ' +1 l singlet (seal quarks. This interpretation is not essential, however. and all we actually demand of Eqs. 10.1 is that ther be suffeiently general. In our standard ft. we fis $a=0$. $\gamma=8$, and $\delta=5$. The rariation of these parameters is discussed below. The parameters $\alpha .3$. $A$. $B$, and $A$ are fitted. The paraueter $C$ determines the fraction of the nucleon's moment um that is carried by gluons. With the other parameters knona. $C$ is fixed by normalization of the fractional momentum distributions

$$
\int_{0}^{1}\left[\frac{18}{5} F_{2}(x)+G(x)\right] d x=1
$$

*hich gives

$$
C=(1-\cdots)\left\{1-\frac{18}{5}\left[A B(1+\alpha \cdot 1+3)\left(1+\frac{a(1+a)}{2+a+3}\right)+\frac{B}{1-\delta}\right]\right\} .
$$

$B$ is the beta function. $B(z, u)=\Gamma(z) \Gamma(u) / \Gamma(z+u)$. We flt the pure singlet case - no correction is made for the neutron excess in iron. 
The result of the standard fit applied simult aneously to the 215 and $93 \mathrm{GeV}$ measurements is show in Fig. 10.3. The family of curves traces the $Q^{2}$ variation of $F_{2}$ at various values of $x$ from the single global flt. We llnd a value for $A$ of $225 \mathrm{MeV} / \mathrm{C}$ with a statistical error of $43 \mathrm{MeV} / \mathrm{c}$. The values of the other fited parameters and their statistical errors are listed as entry (a) of Table 10-II. Each error represents the allowed excursion of the parameters for a unit increase of $\gamma^{2}$. The marginal $\chi^{2}$ of the fit (15.1/91 degrees of freedom) implies that this is an underestimate of the actual uncertainties, either due to the inadequacy of lowest order QCD, to our structure of assumntions, or to the presence of systematic errors. As discussed below, the uncertainties due to these possibilities outweigh those of a purely statistical origin. Entries (b)-(d) of Table 10-II show the minor effects of changing the form of the paranet rization. The parameters are suffiently correlated to allow a restructuring of the parametrization to give searly the same $F_{2}\left(I, Q_{0}^{2}\right)$, without affecting $\mathrm{A}$. Changing $Q_{0}^{2}$ from 5.535 to $25 \mathrm{GeV}^{2} / \mathrm{c}^{2}$ has no effect on $\mathrm{A}$.

We can ask whether the other fited parameters give us any physically meaningful information. The experimental distinction between the charged quarks and the netriral gluons is clear. From the integral of the fitted distributions, we find that $48{ }^{\circ} \mathrm{c}$ of the nucleon's momentum is carried by gluons at $Q_{0}^{2}$. The separatioc between valence and sea quarks in our case is carried entirely by the parametrization. If we choose to interpret the second term of the $F_{2}$ parametrization (Eq. 10.1) as representing the sea, the standard fit tells us that $16^{c} c$ of the momentum of tre target nucleon is carried by quark-antiquark pairs. However, an immediate problem occurs with the subsequent identification of the first term as the valance quark contribution. If we remove the charge-squared factor and one pow' $\mathrm{r}$ of $I$ from this term, we get back the "ralence" quark moment um distribution. Integrating ?his over $I$ counts the ralence quarks. This is a peaker (model dependent) version of the Gross-Llewellyn Smitb sum rule for $F_{3}$ in neutrino scattering. ${ }^{58}$ Infort unately, performing these operations with the result 5 of our standard fit gives 2.04 valence quarks instead of three. If we constrain the parameter $A$ in Eq. 10.1 to give three valence quarks. we get the fit labeled (e) in Table I0-II. The sea momentum fraction in this fit is $11 r_{r}: 1$ and the gluon fraction are virtually unchanged. Even with this constraint, my confidence in this valence-sea separatinn is minimal. On the ot her band, the constrained resuits are in remarkably precise agreement with more direct neutrino results. ${ }^{20}$ 'sing the neutrino's ability to distinguish between quarks and antiquarks, an antiquark structure function $\bar{q}(2)\left(\approx \approx \frac{1}{2} \times\right.$ the sea $)$ can be measured. With this additional input, the neutrino mensurements yield a giuon moment um

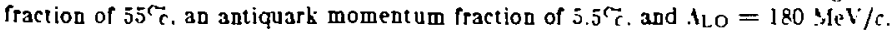

\subsection{Systematic uncertainties}

We deflne systematic uncertainties to menn uncertainties whose origins are purely experimental and affect the measured cross section. Lncertainties that affect the interpretation of the measured cross section. say in terms of 1 . mill be discussed in the nest secrion. Veither the rstimation nor the presentation of systematic eJects is an exact science. We begin by making a catalog of possible sources of experimental uncertainty. tu example would be the MMS magnetir feld calibration. We next estimate the magnitude of the uncertainty in each source and how it would affect our analysis. We then reanalyze the data with each 50":ce in turn changed to reflect its uncertainty, and we observe the effect on our measured and btted $F_{2}\left(x Q^{2}\right.$ ). We also make various tests of internal consistency to reveal possible problems. The changes to $F_{2}\left\{x, Q^{2}\right\}$ from systematic effects are typically correlated. Thus assigning errors to individual measured points is misleading. Sbowing bands of uncertainty on our $F_{2}$ plots is also unsatisfactory - the top of one band may correspond to the bottom of awotber. We will tbus give only a representative example 


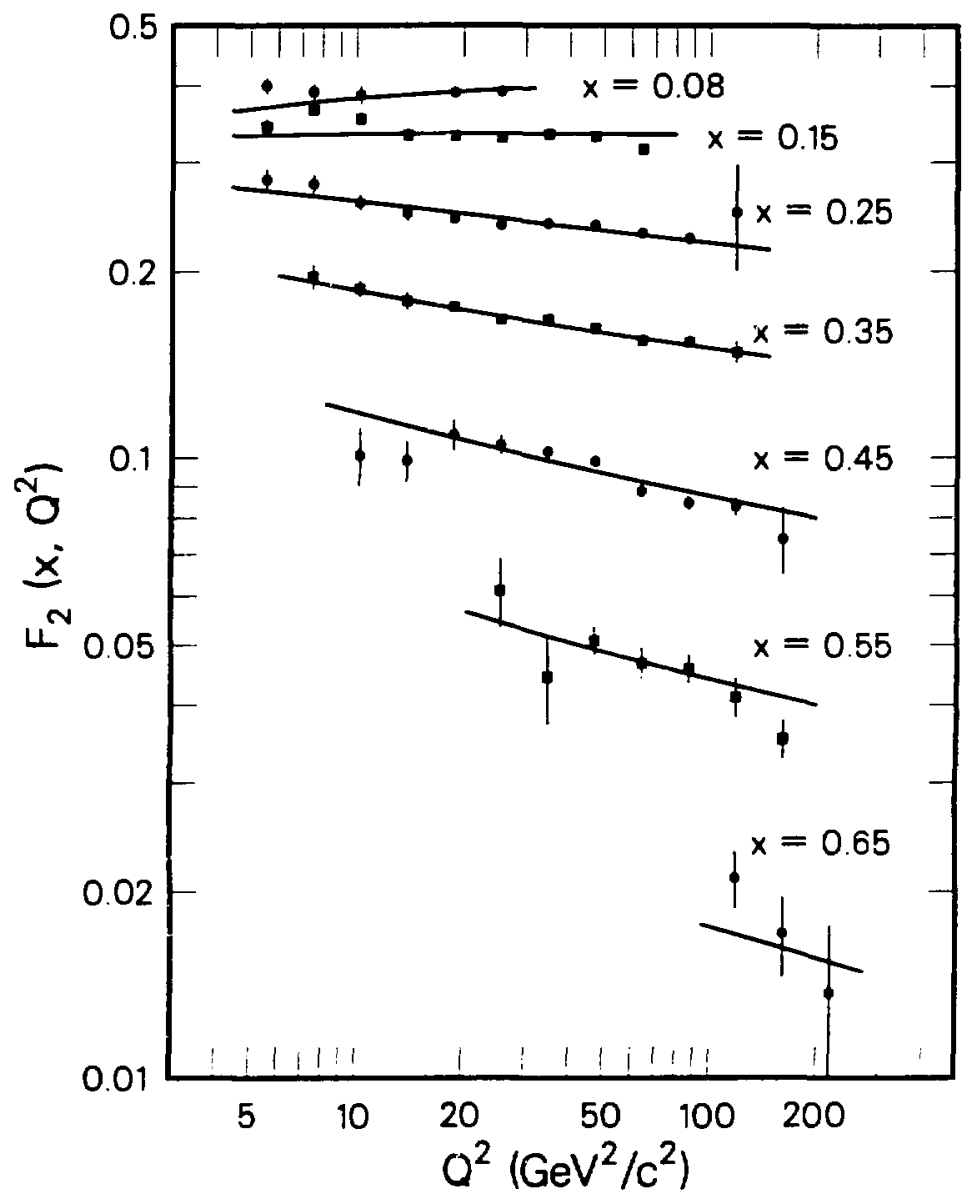

XCG 8311.4903

Fig. 10.3. Results of the lowest-order $Q C D$ st applied simult aneously to the 93 and $215 \mathrm{GeV}$ meacurements of $F_{2} \mid x, Q^{2}$. These measirements have been areraged atier fitting. The curses represent the $Q^{2}$ dependence of $F_{2}$ at barious fxed mlues of $I$ predicted by the siagie global $\mathrm{dt}$. 
Table 10-I. Results of lowest order Q(DD fits to $F_{2}\left(x, Q^{2}\right) . F_{2}\left(x, Q_{0}^{2}\right)=A x^{\alpha}(1-x)^{\beta}(1+a x)+$ $B(1-\rho)^{2} ;\left(j\left(x, Q_{0}^{2}\right)=C(1-x)^{b} ; a, \gamma, \delta\right.$ IIxed; $C$ from momentun sum rule; $\alpha, \beta, A, B$, and $A$ fltted. lirrors are statistical and represent $\Delta x^{2}=1$ excursions in the fit.

\begin{tabular}{|c|c|c|c|c|c|c|c|c|}
\hline Fit & $\Lambda(\mathrm{A} / \mathrm{e} \mathrm{V} / \mathrm{r})$ & $\alpha$ & $\beta$ & $A$ & $B$ & $a$ & $x^{2} /$ dot & Comment \\
\hline $\begin{array}{l}\text { a. Std: } \gamma=8 \\
b=5, a=0\end{array}$ & $225 \pm 43$ & $\begin{array}{r}1.00 \\
\pm 010\end{array}$ & $\begin{array}{r}3.80 \\
+0.15\end{array}$ & $\begin{array}{r}2.74 \\
\pm 0.4\end{array}$ & $\begin{array}{r}0.410 \\
\pm 0.030\end{array}$ & - & $154 / 91$ & $Q_{0}^{2}=5.535 \mathrm{GeV}^{2} / \mathrm{c}^{2}$ \\
\hline b. Wie a & $225 \pm 43$ & 0.51 & 3.97 & $0.8 \cdot 1$ & $0.9 \cdot 48$ & 3.2 .4 & $153 / 90$ & \\
\hline c. $\gamma=12$ & $230 \pm 44$ & 0.71 & 3.56 & 1.92 & 0.350 & - & $153 / 91$ & \\
\hline J. $\alpha=B=0$ & $217 \pm 43$ & - & 3.4 .3 & 0.37 & - & 3.73 & $160 / 92$ & \\
\hline $\begin{array}{l}\text { e. Sum rule } \\
\text { normalization }\end{array}$ & $222 \pm 43$ & 0.67 & 3.35 & $1 . t i 1$ & 0.265 & - & $164 / 92$ & \\
\hline $\begin{array}{l}\text { f. Fit } 93 \mathrm{GicV} \\
\text { normalization }\end{array}$ & $176 \pm 43$ & 0.99 & 3.8 .3 & $2 . t \bar{T}$ & 0.409 & - & $148 / 90$ & $\begin{array}{l}\text { normalization lowered } \\
\text { by } 2.9 \pm 0.8^{\circ}\end{array}$ \\
\hline $\begin{array}{l}\text { g. Standard, EMiC } \\
\text { (lata (Ref. 6i) }\end{array}$ & $159 \pm 25$ & 0.65 & 3.29 & 1.11 & 0.258 & - & $230 / 153$ & $Q_{0}^{2}=5.50 \mathrm{GeV}^{2} / \mathrm{c}^{2}$ \\
\hline
\end{tabular}


Table 10-III. Systematic uncertainties in $\Lambda$. The total indicates the sum of the other entries in quadrature.

\begin{tabular}{|l|c|c|}
\hline \multicolumn{1}{|c|}{ Source } & Uncertainty & $\delta \AA(\mathrm{MeV} / \mathrm{c})$ \\
\hline MLIS B-field calibration & $0.5 \%$ & $<10$ \\
Beam energy & $0.5 \%$ & 10 \\
Trigger efficiency (systematic) & $0.5 \%$ & 16 \\
Trigger efficiency (statistical) & (see test) & 10 \\
Pesolution smearing & (see text) & 50 \\
$9^{\sim}$ '215 GeV normalization & $2.5 \%$ & 60 \\
\hline \multicolumn{2}{|c|}{ Total } & 82 \\
\hline
\end{tabular}

of the efiect on $F_{2}\left(x, Q^{2}\right)$, but concentrate on the global effects on cur fitted results, notably on A. Our catalog of systematic effects is listed in Table 10-IIl.

The first source of systematic uncertainty to be cha-ked was the MMS magnetic field calibration. From the evidence presented in Sec. 7.2 we conclude that the uncertainty in the feld is, conservatively, $\pm 0.5 \%$. What would the effect of a $0.5 \%$ miscalibration be? Since both the beam system and the calorimeter were calibrated to the spectrometer, the result is an overall shit in the measured energy scaie. This would be easy to simulate - change the incident beam energy and MAS ficld by $0.5 \%$ in the simulation and analyze with the original values - but timeconsuming and expensive. Instead, we reason backwards from the existing simulated events, a procedure to be used often in this section. By using the existing simulated events we have flxed the trajectory, that is, the angle, currature and triggering probability of earh track in the MaSS. We reinterpret the true moment !!m of both the incideit and scattered tracks in accordance with the new assumed field value. If $\bar{B}=\epsilon B$ (with $\epsilon=1.005$ ), we find $\overline{Q^{2}}=\epsilon^{2} Q^{2}, \bar{x}=\epsilon x$, and $\bar{y}=y$. Had we actually redone the simulation, this change in kineinatics would bave caused a corresponding change in the cross section for each event. We therefore assign each simulated event $a$ new true $x$ and $Q^{2}$ and a weight given by

$$
\frac{\overline{d \sigma}}{d \sigma}=\frac{1}{\epsilon^{3}} \frac{F_{2}\left(\epsilon x, \epsilon^{2} Q^{2}\right)}{F_{2}\left(x, Q^{2}\right)}
$$

as implied by ig. 8.5. This method is not exact. Radiative corrections, MCS, etc. are left at the old energy scale and $\bar{x}=\epsilon I$ must break dow $n$ at the kinematic limit. For small adjustments in $B$, however, it sbould be adequate. It has the advantage that by using the same events, the modified results are highly correlated to the original set, and thus small changes are readily visible in spite of statistinal fluctuations. The most notable change caused by the adjustment in $B$ is the shift in normalization. This, as well as the other effects of this section, bas been taken into account in the estimate of the normalization nncertainty quoted earlier. The changes in shape are minor. as expected from an overall scale change. As indicated in Table 10-III, the uncertainty in $A$ from this source is less than $10 \mathrm{MeV} /:$.

We expect more substantial effects from a differentia) shift of the beam energy (measured exclusively in the beam system) relatire to that of the scattered track. The precision of the calibration of beam to spectrometer rules out such a relative shift of greater than a few tenths of a percent. We consider a total uncertainty of $\pm 0.5 \%$ to allow for uncertainty due to the discrepancs between the two MMS polarities and for possible effects in the analysis of the average 


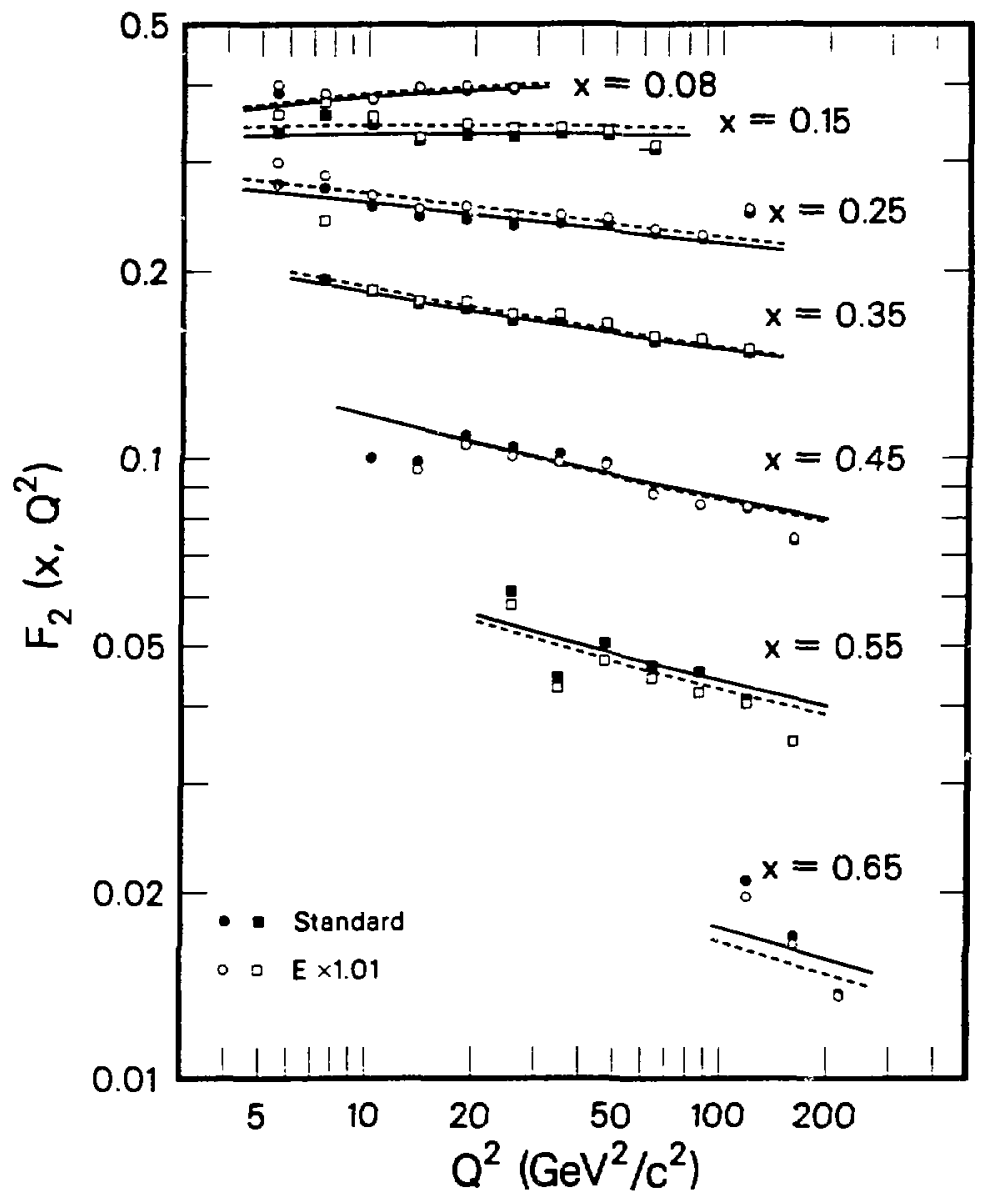

XCG $8311-4900$

Fig. 10.4. Srstematic effects of $1 \%$ shift in beam energy $\approx \approx 2 \times$ the estimated uncertainty). The normalization of the $93 \mathrm{GeV}$ data bas been allowed to toat in each case. The solid (dashed) curves are the QCD fit to the standard (shifted) data. The difference in $A$ between the fits is $20 \mathrm{MeV} / \mathrm{c}$. 


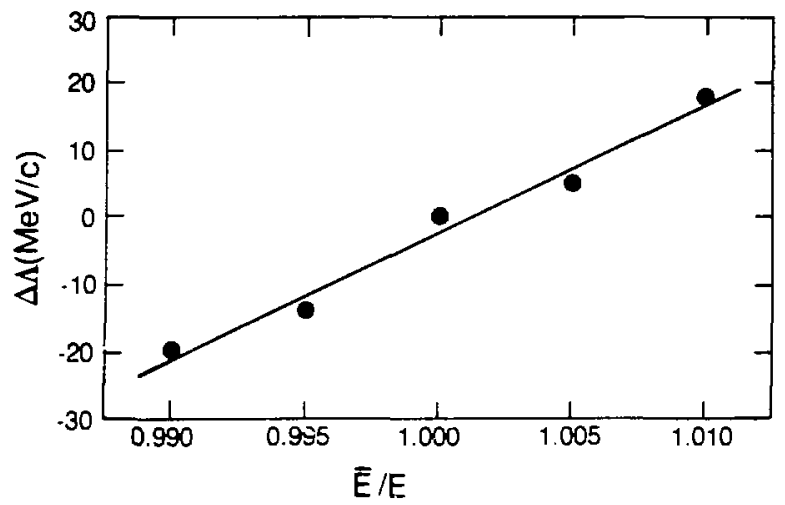

XBL. 8311.763

Fig. 10.5. The dependence of $A$ on systematic error in the beam energy calibration relative to that of the MLSS. The estimated uncertainty is 0.005 . The line is a fit to the points ith slope $(19 \mathrm{MeV} / c)$ per $\left(1 \sigma^{-}\right.$shif $)$.

energy loss of the beam muon en route to the verter. The effect of beam energy shiths was determined in the same fashion as the previous case. To use existing events, we neglect the shift of less than $1 \mathrm{~mm}$ in the vertes position and have $\bar{E}=\epsilon E, \bar{E}^{\prime}=E^{\prime}$, and $\bar{\theta}=\theta$. This gives $\overline{Q^{2}}=\epsilon Q^{2} \cdot \bar{y}=1-(1-y) / \epsilon \cdot \bar{I}=x y / \bar{y}$, and

$$
\frac{\overline{d \sigma}}{d \sigma}=\frac{1}{\epsilon}\left(\frac{y}{\bar{y}}\right)^{2} \frac{\left(1-\bar{y}+\bar{y}^{2} / 2\right)}{\left(1-y+y^{2} / 2\right)} \frac{F_{2}\left(\bar{x}, \overline{Q^{2}}\right)}{F_{2}\left(x, Q^{2}\right)}
$$

Figure 10.4 shows the effect of such an energy shift. magnifled to $\epsilon=1.01$ for clarity. We bare allowed the $93 \mathrm{GeV}$ normalization to adjust to that of the $215 \mathrm{GeV}$ data. As mentioned above, the corresponding points of each set are highly correlated and only their separation is meaningful. The error bars have thus been suppressed. The curves show tne fit to each case. They also demonstrate the effect of changing $A$ in this case by $20 \mathrm{MfV} / \mathrm{c}$. on the slope of $F_{2}\left(Q^{2}\right)$. Figure 10.5 shows the response of 1 to beam energy miscalibration. Our estimate of the uncertainty in $A$ from this source is $\pm 10 \mathrm{MeV} / \mathrm{c}$.

After mapping the effliency of the trigger counters, there are residual uncertainties. both statistical and systematic. We can estimate tbe global srstematic uncertainty in the trigger effliency br comparing events that trigger in different parts of the spectrometer or erents that penetrate different numbers of paddle counters. We feel that any global effieney offset is limited 
to $\pm 0.5 \%$. Even a uniform offset in efficiency can affect the shape of the cross section because the number of paddles penetrated, and thus the sensitivity to the efflciency of individual paddles. varies with $Q^{2}$. We find that a $0.5 \%$ change in efficiency changes $A$ by $16 \mathrm{MeV} / \mathrm{c}$. Even if there is no systematic offset in the efficiency maps, the statistical errors in the maps may affeer our results. In any kinematic region with a large geometrical acceptance, the statistical errors in many map cells are averaged and there is no effect. However, at low $Q^{2}$ oniy small portions of the spectrometer are illuminated and only a limited anount of averaging iakes place. This was the problem with the penultimate set of maps, which were not systematically biased, but had large statistical errors in the crucial regions. We probed this possibility in the new maps by varying by one statistical standard deviation the efficiency of the single most critical map cell (or rather. the corresponding cells in each paddle of trigger bank 6). From this worst case, we estimate that the statistical uncertainty in the efficiency maos leads to an uncertainty in $A$ of $\pm 10 \mathrm{MeV} / c$. As an vverall check, we compare results estracted separately from the events entering the four quadrants of the apparatus as seen by the beam. In Fig. 10.6, we show $A$ as separately fitted in the four quadrants using our $215 \mathrm{GeV}$ data. This figure should be considered in the lignt of Fig. 7.5, wich indicates how different the quadrants actually are. Another indication of the sensitivity of this test is to note that with our previous set of maps, which for two years was believed to be quite adequate, $\Lambda$ from the various quadrants raried by over three orders of magnitude.

Another source of systematic uncertainty is resolution smearing. or rather our ability to model and correct for it. We include here effects due to the use of the calorimeter for resolution improvement. and thus possible calorimeter miscalibration. We study this by varying the degtee to which the calorimeter is used. We relas the missing energy requirement (see Sec. 6.4) and use the calorimeter in all events (witb the same weighting scheme as before). We also try ignoring the calorimeter information totally. The kinematic cuts described in the previous chapter were chosen to minimize the effects of these changes. The uncertaint $y$ in $A$ from this source is estimated to be $\pm 50 \mathrm{MeV} / \mathrm{C}$.

The major source of uncertainty in $A$ is the relative normalization of our 93 and $215 \mathrm{GeV}$ data sets. Since $A$ determines the slope of the $Q^{2}$ dependence of $F_{2}$ at fixed $I$, and cur low $Q^{2}$ data comes from one set and bigh $Q^{2}$ from the other, this is no surprise. We estimate the uncertainty in the relative normalization to be $\pm 2.5-c$. This causes a $\pm 60 \mathrm{MeV} / \mathrm{c}$ uncertainty in $\mathrm{A}$. We can also allow the relative normalization to float in the global fit. Table 10-I, entry (f) shows the result of this fit. The result is to lower the $93 \mathrm{GeV}$ normalization by $2.0 \pm 0.8 \%$. within the estimated uncertainty. Alternatively, we can normalize directly by comparing the 15 points where thc data sets overlap. The flrst normalization check is, of course, model dependent: the second is more sensitive to $R$. The direct normalization indicates that the $93 \mathrm{GeV}$ event sample should be increased by $2.9 \pm 1.5 \%$. Eliminating the same two points that contributed the most to the $\chi^{2}$ of the comparison between the two data sets reduces the normalization shift to $1.5 \pm 1.5 \%$. Unless otherwise indicated. all results of the chapter refer to the original (separate. absolute) normalizations, including the corrections diseussed in the previous chapter.

As another consistency check. we can separately flt the 93 and $215 \mathrm{GeV}$ measurements. Infortunately. the resulting smaller $Q^{2}$ ranges and our limited $93 \mathrm{GeV}$ statistics reduce the significance of this comparison. The results are not terribly satisfying. The fit of the $93 \mathrm{GeV}$ data gives $\mathrm{A}=633 \pm 148 \mathrm{MeV} / c, 2.6$ statistical standard deviations higher than the $215 \mathrm{GeV}$ value of $227 \pm 55 \mathrm{MeV} / \mathrm{c}$. Inspecting. Fig. 10.2, it is apparent that the slopes of the $93 \mathrm{GeV}$ data are somewhat steeper. In fact, there has been some attempt ${ }^{80}$ to attribute physical significance to a similar effect in the data of the European Muon Collaboration (though not by the experimenters). My opinion is that systematic errors must be reduced in all of the experiments before such effects are 10 be considered significant. On the positive sid the agreement between the result of the combined fit and that of the $215 \mathrm{GeV}$ data alone is excellent. The effects of shifting the relative 


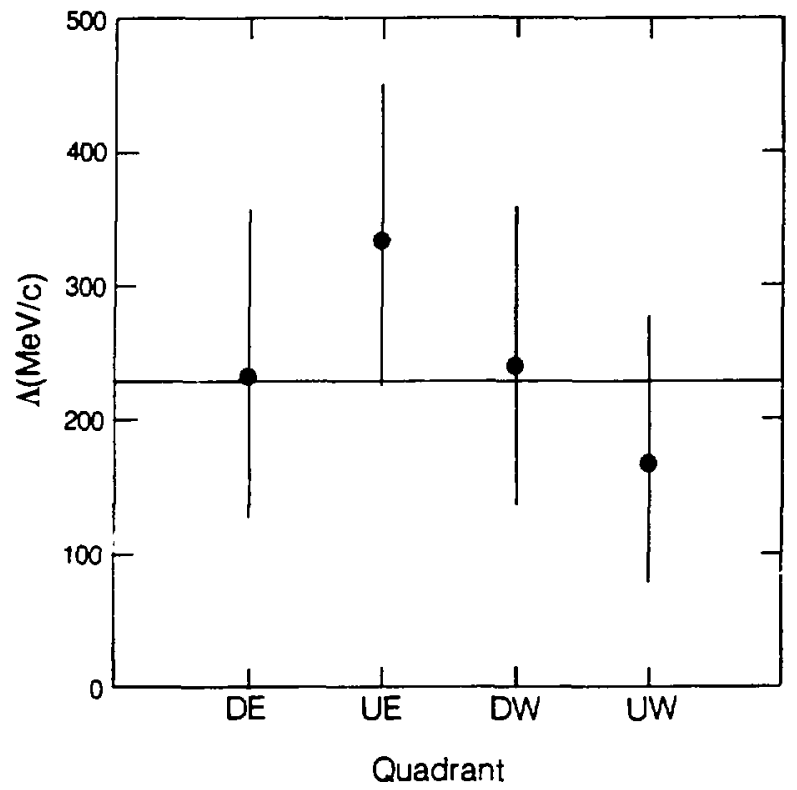

XPL 8311.764

Fig. 10.6. $\mathrm{A}$ by quadrant. The $215 \mathrm{GeV}$ data has been sorted by quadrant and $F_{2}\left(x, Q^{2}\right)$ extracted and fit separately for each. The line shows the value of $A$ fit to the total $215 \mathrm{GeV}$ sample (227 $\mathrm{MeV} / \mathrm{c})$.

normalization indicate that this agreement is not simply due to the $215 \mathrm{GeV}$ data dominating the combined fit.

Table 10-II concludes with an estimate of our total systematic uncertainty in A of $82 \mathrm{MeV} / c$, the sum in quadrature of the indiridual entries. This estimate is obviousiy accurate only to the ertent that our catalog of sources is complete. It is apparent, borever, that the șstematic uncertainty already dominates the statistical uncertainty. 
Table 10-rV. Effects of various phenomenological assunptions on the fitted ralus of $A$.

\begin{tabular}{|l|c|c|}
\hline \multicolumn{1}{|c|}{ Assumption } & $\Delta \Lambda(\mathrm{MeV} / \mathrm{c})$ & $\chi^{2} / \mathrm{dof}$ \\
\hline Standard & $(\Lambda=225)$ & $154 / 91$ \\
$R=0.1$ & -100 & $142 / 91$ \\
Fermi motion correction & $+20 \mathrm{to}+60$ & 149 to $151 / 91$ \\
$x>0.15$ & +5 & $134 / 81$ \\
Gluon exponent $=6$ & -25 & $152 / 91$ \\
Higher order $Q \mathrm{CD}$ & +50 (estimated) & - \\
Target mass correction & +15 & $155 / 91$ \\
Include $1 / Q^{2}$ term & -25 or more (see text) & $152 / 91$ \\
\hline
\end{tabular}

\subsection{Phenomenological uncertainties}

Beyond the experimental uncertainties discussed in the previous section lie questions of interpretation. We bave measured $A$ by assuming that the cross section (Eq. 8.5 with $R=0$ ). the parametrization (Eq. 10.1), and lowest order perturbative QCD are a complete description of deep inelastiz scattering. In this section we explore the effects of relasing or varying the various pieces of this assumption. Table 10-WV summarizes the results.

We start with the cross section and begin by varying $R$. In this exercise, the measured cross section remains constant; the measurement is simply apportioned differently between $F_{2}$ and $R$. We choose $R=0.1$ as an upper limit based on the measurements of $R$ to be discussed below. Even this soall change decreases $\mathrm{A}$ by $100 \mathrm{MeV} / \mathrm{c}$.

Another matter of interpretation concerns Fermi motion As we have defined it in Eq. 8.5, our measured $F_{2}\left(x, Q^{2}\right)$ refers to an average over the nucteons in an iron nucleus. To translate this into $F_{2}$ for a free nucleon, we can try to remove tbefinown effects of the nuclear environmeni such as binding energy and jts manifestation as Fermi motion. This correction has been demoted in importance by the discovery ${ }^{61}$ of another nucjeus-dependent effect which is larger in the I region covered by our data. This "EMC effect" is discussed in the nert section.

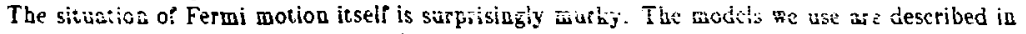
Appendix B. In brief. Fermi motion is primarily an $x$ dependent pbenomenon, and thus bas a limited effect on $A$. Depending on the model used, we find that $A$ increases by 20 to $60 \mathrm{MeV} / \mathrm{c}$ when Fermi motion corrections are applied. Above the very low $Q^{2}$ region $\left(Q^{2}<2 \mathrm{GeV}^{2} / \mathrm{c}^{2}\right)$. the ENC effect has no observed $Q^{2}$ dependence. ${ }^{.2}$

Our dats ertend into the kinematic region known to contain scaling violations due to the crossing of the charm tbreshold. ${ }^{0}$ We have made no detailed attempt to correct for this. but rather try removing the region in question and observe the effect. Before the interpolation to $z$ bin centers. we eliminate all measurements with $z<0.15$. This results in an increase in 1 of only $5 \mathrm{MeV} / c$.

We have already discussed the minor effects of varying some of the more arbitrary features of our parametrization at $Q_{0}^{2}$. One feature. however. has an unambiguous pbysical interpretation. This is the exponent $\delta$ which determines the "hardness" of the gluon momentum spectrum. In inclusive muon scattering we cannot directly measure the gluons. This is unfortunate, because it has been noted ${ }^{\mathrm{t3}}$ that $\mathrm{A}$ and this exponent can be strongly correlated. As 


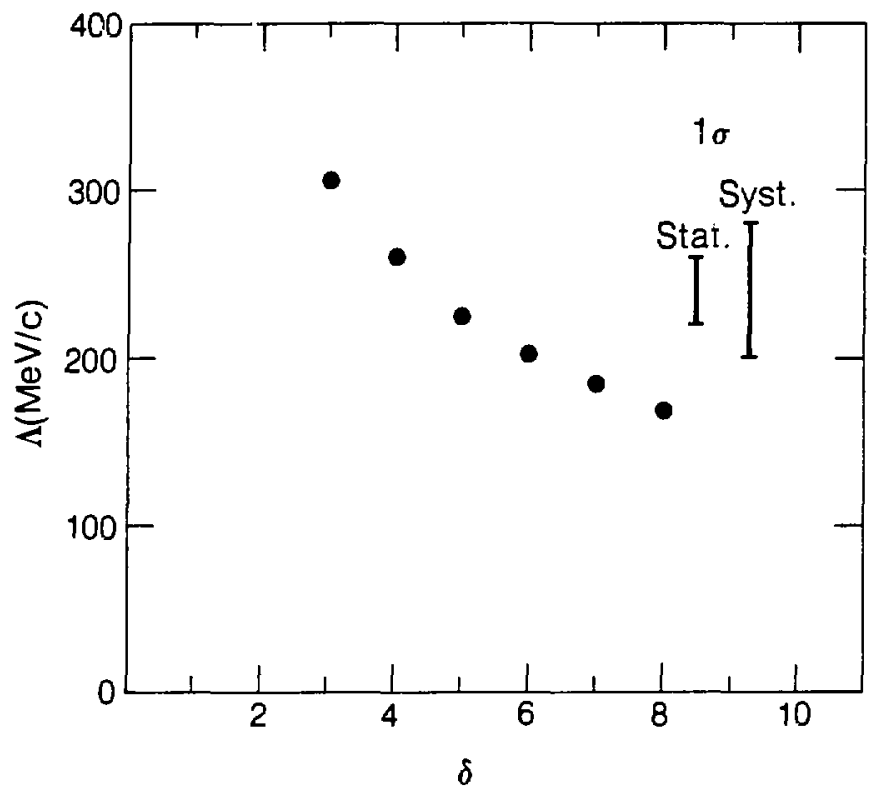

XBL $8310-738$

Fis. 10.7. The correlation between our best-flt value of $\Lambda$ and the value assumed for $\delta$, the exponent of $(1-x)$ in the gluon distribution. For comparison, the statistical and systematic uncertainties in our standard bit of $\Lambda$ are show $\mathrm{n}$. 
mentioned earlier, the non-singlet information arailable from neutrino scattering allows a more constrained determination of the gluon sector. The CERN-Dortmund-Heidelberg-Saclay (CDHS neutrino collaboration ${ }^{2 \theta}$ has found the exponent $\delta$ to lie in the range 5 to $T$, but the actual precision of this result has been questioned. ${ }^{64}$ Our standard fit uses $\delta=5$. Raising this to $\delta=6$ decreases $A$ by $25 \mathrm{MeV} / c$. Figure 10.7 traces further the dependence of our best fit value of $A$ on the assumed value of $\delta$.

As discussed in Chap. 3, our lowest order QCD fits neglect the contribution of higher powers of $\ln Q^{2}$ and powers of $1 / Q^{2}$ to the $Q^{2}$ dependence of $F_{2}$ at fixed $x$. This is known as the "leading-log, leading twist" approximation. The rext-to-leading-log contribution can be incorporated into the Altarelli-Parisi evolution equations, but we have not done so. From the work of others ${ }^{64,05}$ we estimate that these higher order corrections increase $A$ by approximately $50 \mathrm{MeV} / \mathrm{c}$. The $1 / Q^{2}$ terms are, in general, not calculable. An exception is the "target mass correction" $w$ bich can be taken into account ${ }^{86}$ by replacing the variable $x$ with

$$
\xi=\frac{2 x}{1+\sqrt{1+4 x^{2} M_{N / Q^{2}}^{2}}} .
$$

In our $Q^{2}$ and $I$ range, this effect should be small. When this transformation is made we flad that $\Lambda$ increases by $15 \mathrm{MeV} / \mathrm{c}$. Considerably more freedom in $\Lambda$ is allowed if we inciude an arbitrary $1 / Q^{2}$ dependence in our parametrization. We frst simply multiply our $F_{2}$ parametrization by $\left(1+k / Q^{2}\right)$ with $k$ determined by the fit. $\Lambda$ drops by $100 \mathrm{MeV} / c$, with a moderate improvement in $x^{2}$ from $154 / 91$ dof to $130 / 90$ dof. To gain sensitivity, the EMC investigated $1 / Q^{2}$ terms by combining their muon-proton data witb lower $Q^{2}$ electron-proton data from SLAC. ${ }^{85}$ They allowed the $I$ dependence of the $1 / Q^{2}$ term to vary and found that this dependence was consistent with $k x^{2}(11-x)^{2}$. If we use $t$ his form and $f x k=0.45$, which is the approximate level observed by the EMC. $\Lambda$ drops by $25 \mathrm{MeV} / \mathrm{c}$ with a negligible improvement in $\chi^{2}$. If we instead try to use our data to determine $k$, we find $k \approx 4, \Lambda=30 \mathrm{MeV} / c$, and $\chi^{2}=144 / 90 \mathrm{dof}$. It is not clear that anyone is doing anything but quantifying systematic errors with these flts. The fact remains that our experiment alone cannot rule out large $1 / Q^{2}$ corrections to lowest order $Q C D$.

\subsection{Comparison with other experiments}

The most direct comparison we can make is to data from the European Muon Collaboration (EMC) ${ }^{6:}$ Part of their data. was taken on an iron target at beam energies similar to ours. Their spectrometer, however, was completely different from the MMS, and thus we can hope that some of the srurces of systematic error are different for the two experiments. Figure 10.8 shows a comparisor of our results with the EMC's iron target measurements. Both measurements are averaged over the rarious beam energies, both assume $R=0$, and neither has been corrected for Fermi motion. For purposes of this comparison only, our values of $F_{2}$ have been multiplied by a factor of 0.94 , determined from the $I$ dependence comparison discussed below. This normalization is consistent with the $3 \widetilde{c}$ systematic uncertainty quoted by each experiment. Except for the region of $Q^{2}<10 \mathrm{GeV}^{2} / c^{2}$, the agreement is excellent. Table $10-$ Il includes an entry for a fit to the EMC's data using our fitting routine and assumptions. These fitted parameters are consistent with the EMC's published results. ${ }^{85}$ although the assumptions made here are slight!y different. It should be noted that while the individual parameters of $F_{2}(\tau)$ at $Q_{0}^{2}$ appear different in fits $(a)$ and $(\mathrm{g})$, the actual ralues or $F_{2}\left(x, Q_{0}^{2}\right)$ are quite similar after the $6 r_{c}$ shift indicated above.

In Fig. 10.9 the ratio of our $F_{2}^{\mathrm{Fe}}$ (multiplied by 0.94 ) to that of the EMC for $Q^{2}>$ $20 \mathrm{GeV}^{2} / \mathrm{c}^{2}$ is displaşed as a lunction of $I$. Fits $(\mathrm{a})$ and $(\mathrm{g})$ of Table 10 -Il bave been used to 


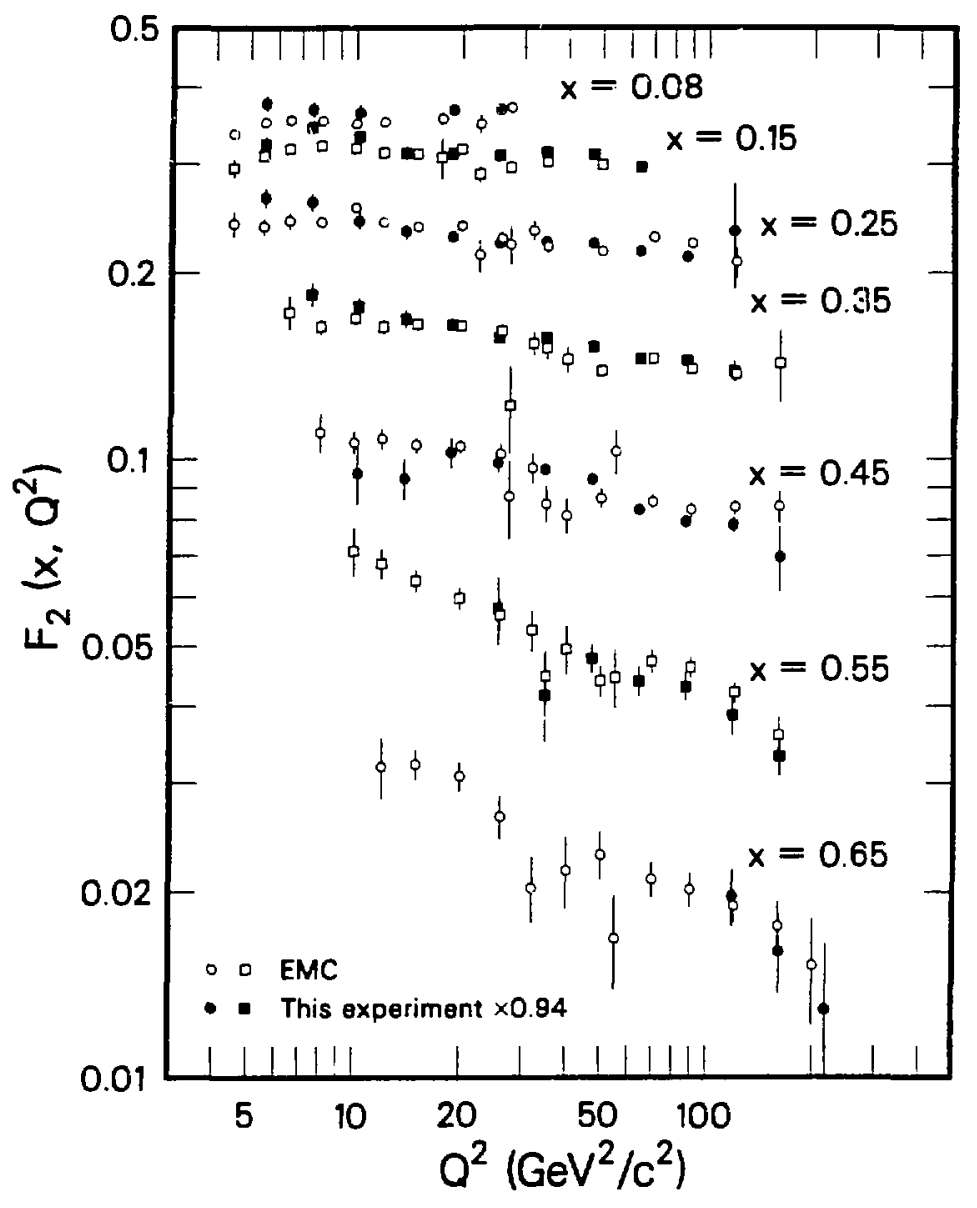

XCG $8311-4904$

Fis. 10.8. A comparison of our measured $F_{2}\left(r, Q^{2}\right)$ (mutiplied by 0.94) to the EMC's iron target measurement (Ref. $6 \pi$.). 


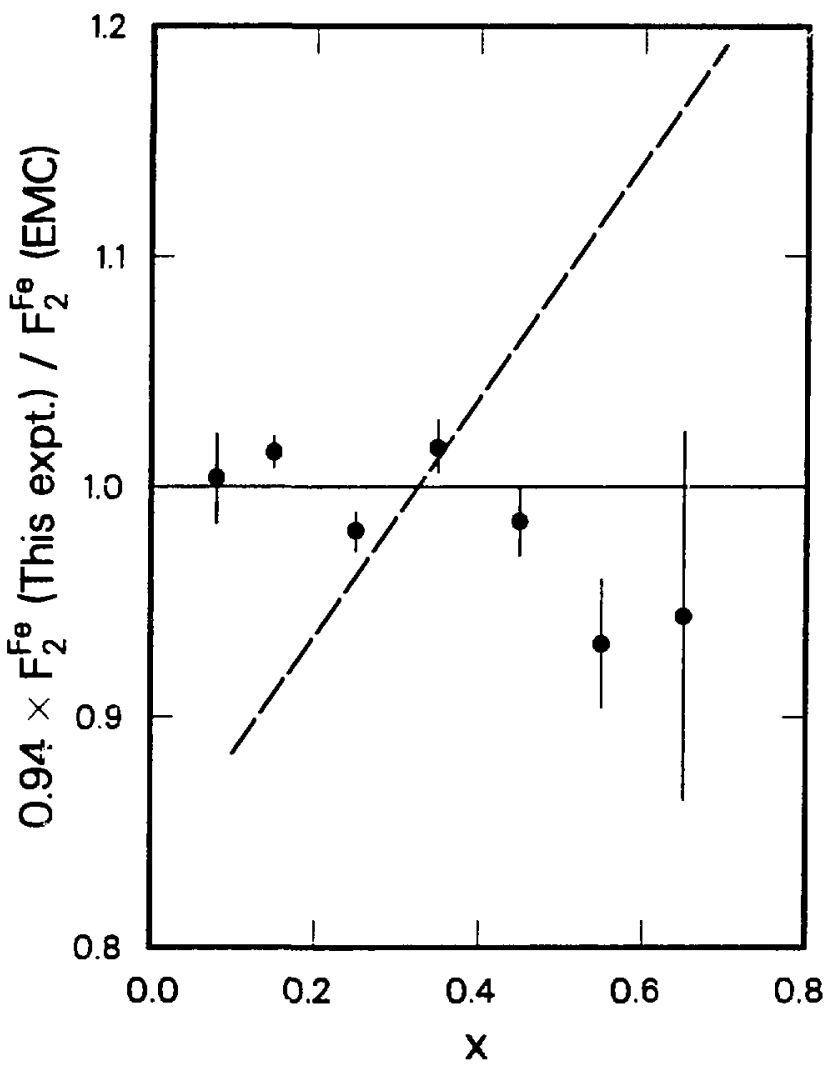

XCG 8311-4805

Fig. 10.9. The ratio of our $F_{2}(\mathrm{Fe})$ measurements (maltiplied by 0.941 to the ExIC"s (Ref. 67.) as a function of $I_{\text {, for }} Q^{2}>20 \mathrm{GeV}^{2} / c^{2}$, interpolated to $Q^{2}=50 \mathrm{GeV}^{2} / c^{2}$. The dashed line shows the irend of EMC's $F_{2}$ (deuterium) $/ F_{2}(\mathrm{Fe})$ from Ref. 61 . 
interpolate each data point to $Q^{2}=50 \mathrm{GeV}^{2} / \mathrm{c}^{2}$ prior te averaging. The errors shown are statistical; systematic uncertainties from our data alone are of comparable magnit ude. The EMIC has reported differences of up to $15 \%$ between deuterium and iron in the $I$ dependence of $F_{2} \cdot{ }^{61}$

These discrepancies, not explained by Fermi motion, have been confirmed using jesurrected data from old experiments at SLAC. ${ }^{62}$ The dashed line in Fig. 10.9 show $s$ the trend of $F_{2}(d) / F_{2}(\mathrm{Fe})$ for the EMC's measurements. We, of course, cannot make the deuterium-iron comparison directly. However, from the evidence of Fig. 10.9, we can provide support for the existence of the discrepancy by noting that our measurements agree quite well with the EMC's iron measurements. but are distinctly different from their deuterium measurements.

We can also compare our results to those of charged current neut rino experiments. Here the comparison is somewhat less direct, due to the difference in the coupling to quirks between the reak and electromagnetic probes used. The parton model predicts that

$$
F_{2}^{\mu N}(x)=\frac{5}{18} F_{2}^{\nu N}(x)+\epsilon(x)
$$

where the $5 / 18$ factor is the average electric charge squared of the quarks and $\epsilon$ represents a residual difference in the coupling to the sea of heary quarks and antiquarks. This $\epsilon$ term, expected to be positive, is complicated by the threshold behavior in the neutrino interaction where an $s$ quark must be turned into a massive $c$ quark. Figure 10.10 shows our results compared to $\frac{5}{15} \times F_{2}^{\nu N}$ from the CDHS collaboration, which also has an iron target. ${ }^{68}$ For the comparison, our $F_{2}$ has been multiplied by 0.89 . Both sets assume $R=0$ and neither has been corrected for Fermi motion. Although CDHS fits a similar value of $\Lambda$ to ours, ${ }^{29}$ the agreement with our $I$ dependence (see Fig. 10.11) is not as good as that between the two muon experiments. The difference at low $z$ is not in the direction expected from the beary quark sea contribution mentioned above. This discrepancy has been noted before ${ }^{6 \theta}$ and will bave to be resolved by future experiments. Figure 10.12 shows the $x$ dependence of the three esperiments, again for $Q^{2}>20 \mathrm{GeV}^{2} / c^{2}$ and interpolated to $Q^{2}=50 \mathrm{GeV}^{2} / r^{2}$, with the arbitrary normalizations mentioned above.

We can compare our measurement of $\Lambda_{L O}=225 \pm 43^{\text {stat }} \pm 82^{\text {syst }} \mathrm{MeV} / \mathrm{c}$ to the results of other investigations of the strong coupling constant $\alpha_{s}\left(Q^{2}\right)$. In $e^{+} e^{-}$annihilation. $\alpha_{S}$ appears whenever gluons are present. For example. the value of $\alpha_{\xi}$ can be determined by observing the modifications due to gluon emission to the two-jet structure of events. There are various metbods of characterizing this modification, but all appear to be subject to substantial uncertainties from the (non-pert urbative) fragmentation of the primary quarks and gluons into the observed hadrons. At a center of mass energy $\sqrt{s} \approx 30 \mathrm{GeV}$, values of $\alpha_{S}$ typically between 0.13 and 0.20 are found. ${ }^{70}$ If we use Eq. 3.11 with $Q^{2}=s$ and $N_{f}=5$ to turn these results into measurements of $A_{L O}$, we find $A_{L O}=60-400 \mathrm{IeV} / c$. The expectation that the hadronic decay of the $\mathrm{T}$ is dominated by by $\mathrm{T} \rightarrow 3$ gluons allows a determination of $c_{3}$ from the observed decay widths of the $T^{71} \quad l t$ is found that $\alpha_{S}\left(H_{T}\right)=0.158 \pm 0.011$. This translates into $1_{\text {LO }}=$ $80 \pm 30 \mathrm{MfeV} / \mathrm{c}$. One of the interpretational diffculties bere is the appropriateness of using. $\mathrm{fT}_{\mathrm{T}}$ as the argument of $\alpha_{S}$. It is apparent that in $e^{+} e^{-}$annihilation. as in deep inelastic scattering. the confrontation of theory with experiment is limited as much by questions of interpretation as by experimental precision. 


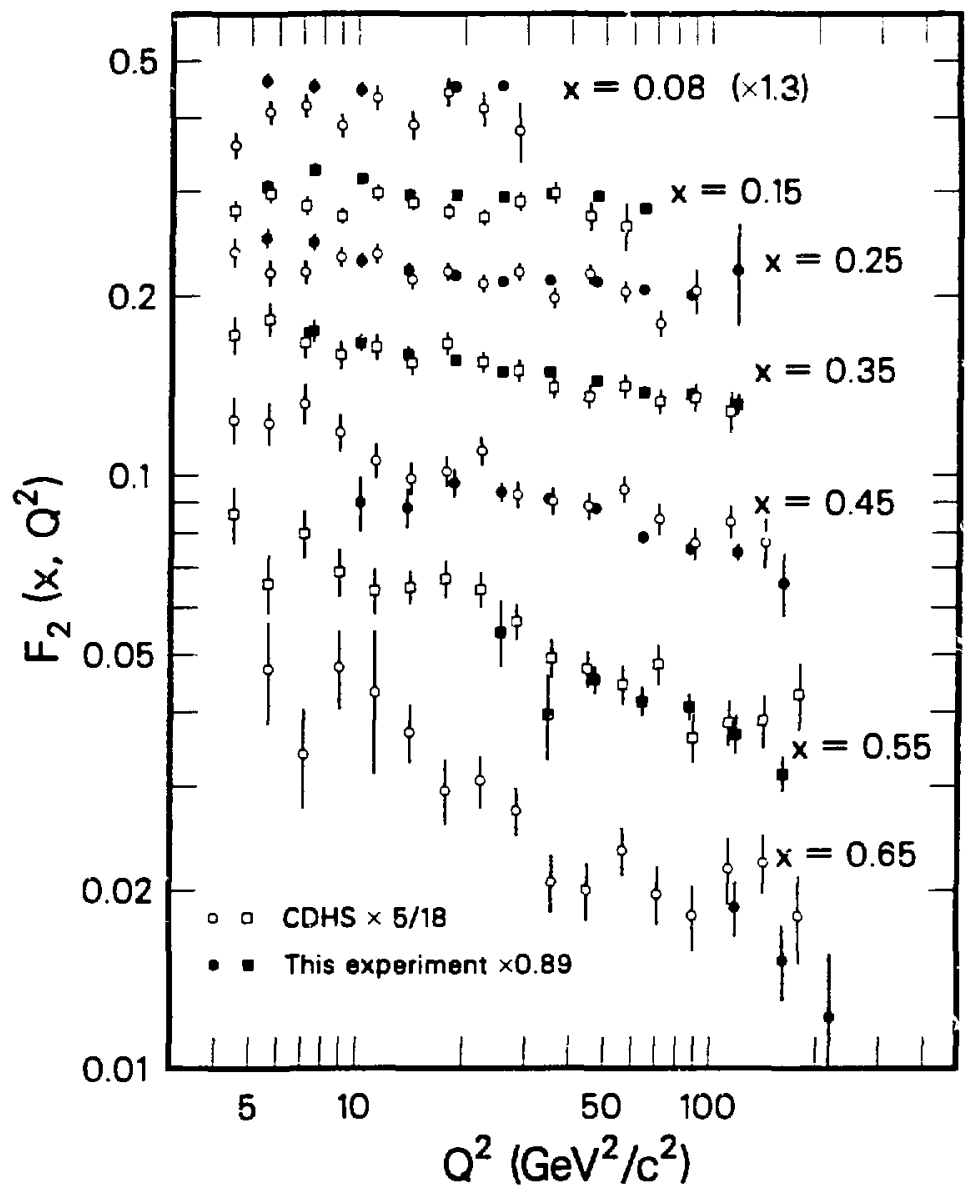

XCG B311-4901

Fig. 10.10. Our measured $F_{2}\left(x, Q^{2}\right)$ (multiplied by 0.89) compared to $\frac{5}{\sqrt{2}} \times$ $F_{2}^{\mu F e}$ from CDES (Ref. 68.). For clarity, the $I=0.08$ bin has been raised by a factor of 1.3 . 


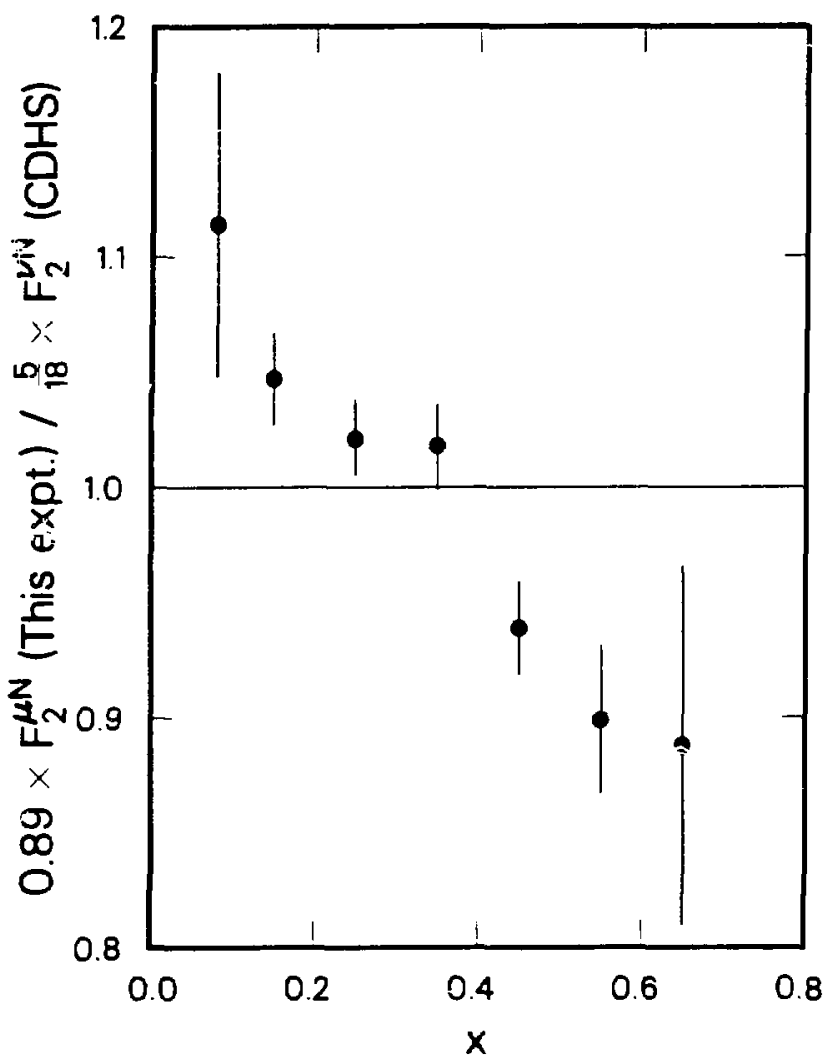

XCG $8311-4800$

Fig. 10.11. A comparison of the $I$ dependence of our $F_{2}^{\mu F e}$ and $x F_{2}^{\nu F e}$ from CDHs (Ref. 68.). Measurements for $Q^{2}>20$ bave been avera,ed after interpolation $10 Q^{2}=50 \mathrm{GeV}^{2} / \mathrm{c}^{2}$. 


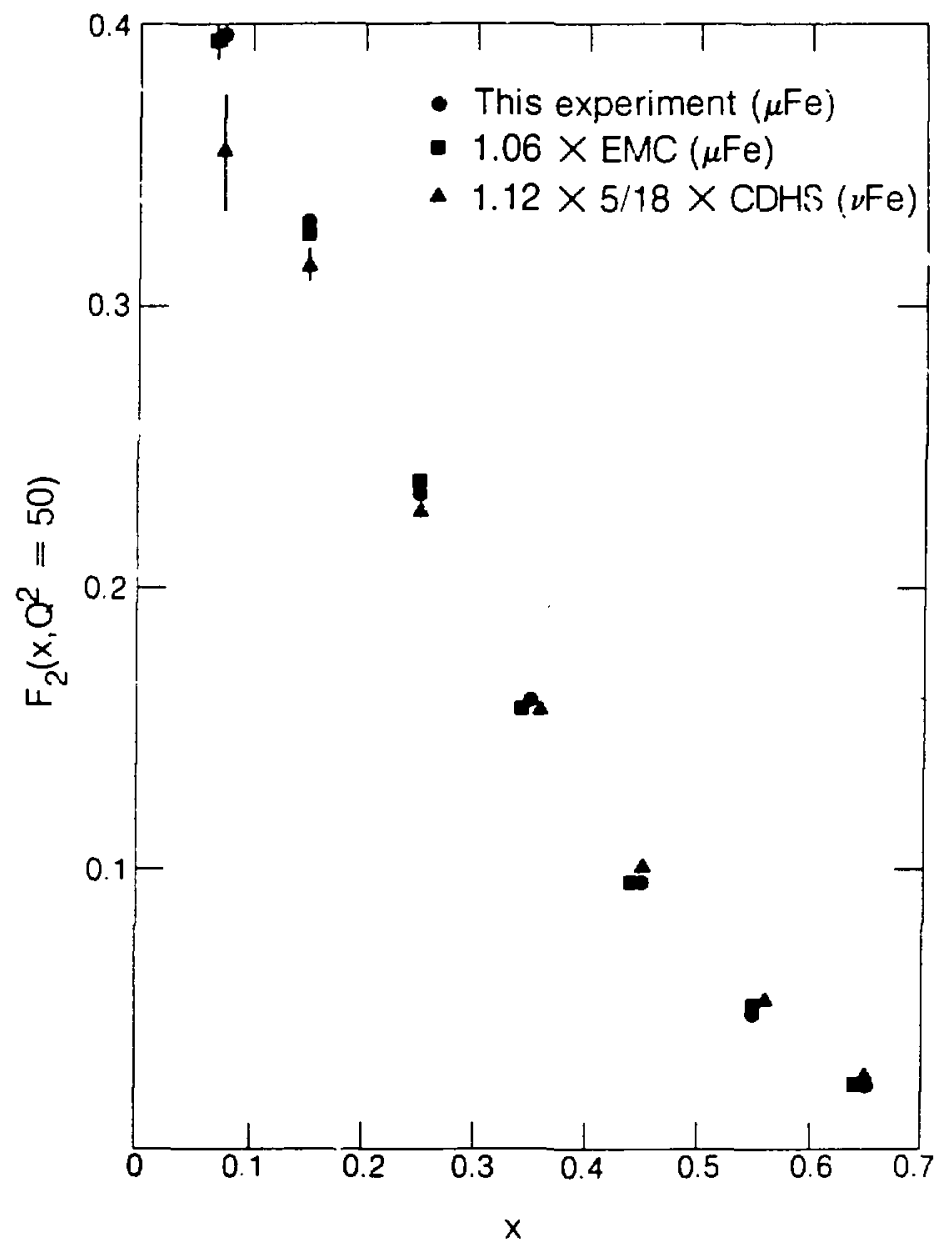

BQL 2311.765

Fis. 10.12. The $I$ dependence of $F_{2}\left(Q^{2}=50\right)$ determined frow our measurements and those of the EMC (Ref. 67.) and CDHS (Ref. 68.). All experiments had iron targets. made no Fermi motion correction, and assumed $R=0$. Some points bare been displaced slightly in a for clarity. 


\subsection{Measurement of $\mathrm{R}$}

Although previous results hare assumed a fixed value of $R \equiv \sigma_{L} / \sigma_{T}$, usually $R=0$, our overlapping measurements of $F_{2}\left(x, Q^{2}\right)$ taken at different bean energies allow us to measure $R$. At flxed $Q^{2}$ and $\nu$ (i.e., at flxed $Q^{2}$ and $x$ ) we have fised the 4-momentum of the virtusl photon, independent of the muon beam energy. However, this does not exhaust the photon's quant um numbers; it also carries polarization. We can isolate the dependence on the virtual photon polarization by writing the differential cross section in two forms:

$$
\begin{aligned}
\frac{d^{2} \sigma}{d Q^{2} d \nu} & =\frac{4 \pi \alpha^{2}}{Q^{4}} \frac{E^{\prime}}{E} \cos ^{2} \frac{\theta}{2}\left(2 W_{1} \tan ^{2} \frac{\theta}{2}+W_{2}\right) \\
& =\Gamma_{T} \sigma_{T}+\Gamma_{L} \sigma_{L} \equiv \Gamma_{T}\left(\sigma_{T}+\epsilon \sigma_{L}\right) .
\end{aligned}
$$

$\Gamma_{T . L}$ and $\sigma_{T, L}$ represent the fluxes and cross sections for transversely and longitudinally polarized virtual photons; the polarization $\varepsilon \equiv \Gamma_{L} / \Gamma_{T}$. Using the expressions for $W_{1}$ and $W_{2}$ in terms of $\sigma_{L}$ and $\sigma_{T}$ (Eq. 2.11), we can identify

$$
\begin{aligned}
\epsilon & =\frac{1}{1+2\left(1+\nu^{2} / Q^{2}\right) \tan ^{2}(\theta / 2)}=\frac{1-(\nu / E)-\left(Q^{2} / 4 E^{2}\right)}{1-(\nu / E)+\left(\iota^{2} / 2 E^{2}\right)+\left(Q^{2} / 4 E^{2}\right)} . \\
\Gamma_{T} & =\frac{K \alpha}{2 \pi Q^{2}} \frac{1}{E^{2}} \frac{1}{1-\epsilon} .
\end{aligned}
$$

$K$ is a factor that must approach $\nu$ as $Q^{2}-0$ to agree with real photoproduction. ${ }^{10,72}$ From this expression for $\epsilon$ we see that, at flsed $\nu$ and $Q^{2}$, varying the muon beam energy changes the polarization of the "beam" of virtual photons.

Inserting $R$ into Eq. 10.8. we define

$$
S \equiv \frac{1}{\Gamma_{T}} \frac{d^{2} \sigma}{d Q^{2} d \nu}=\sigma_{T}(1+\epsilon R)
$$

$R$ is thus the slope of $S$ vs. $\epsilon$. To extract $R$, we recall that our measurement of $F_{2}$ is actualls a measurement of the cross section coupled to an assumption about $R$. By restoring the kinematic factors of Eq. 2.12 with $R=0$ to $F_{2}$, we get a messured cross section independent of $R$. For measurements at different beam energies $E_{i}$, but at the same $\nu$ and $Q_{2}$,

$$
S_{i}\left(x, Q^{2}\right)=\frac{4 \pi^{2} \alpha}{K} \frac{\left(\nu^{2}+Q^{2}\right)}{Q^{2} \nu} F_{2}^{i}\left(x, Q^{2}\right)
$$

where $F_{2}^{i}$ is the measured $F_{2}$ with $R=0$. Lising Eqs. $10.8-11$ fe get a measurement of $R$ for each point where the two data sets overlap:

$$
R\left(x, Q^{2}\right)=\frac{S_{2}-S_{1}}{S_{1} \epsilon_{2}-S_{2} \epsilon_{1}}=\frac{F_{2}^{2}\left(x, Q^{2}\right)-F_{2}^{1}\left(x, Q^{2}\right)}{F_{2}^{1}\left(x, Q^{2}\right) \epsilon_{2}-F_{2}^{2}\left(x, Q^{2}\right) \epsilon_{1}},
$$

where the incices 1 and 2 refer to the 93 and $215 \mathrm{GeV}$ beam energies, respectively.

It is clear that this measurement of $R$ will be sensitive to systematic experimental differences between the dats sets. Beginning with the $F_{2}$ measurements of Table $9-11$, we find 
Table 10-v. $R$ measurements. Errors are statistical. The final entry is a global axerage.

\begin{tabular}{|c|c|c|c|c|}
\hline$I$ & $Q^{2}\left(\mathrm{GeV}^{-2} / c^{2}\right)$ & $f(93 \mathrm{GeV})$ & $(215 \mathrm{GeV})$ & $R$ \\
\hline 0.1633 & 18.18 & $C 5870$ & 0.9464 & $-0.057 \pm 0.085$ \\
0.2421 & 18.31 & 0.8359 & 0.9772 & $-0.160 \pm 0.185$ \\
0.3252 & 18.20 & 0.9184 & 0.9880 & $-0.378 \pm 0.291$ \\
0.3863 & 18.20 & 0.9445 & 0.9916 & $-0.550 \pm 0.325$ \\
0.2437 & 24.73 & 0.6682 & 0.9564 & $0.011 \pm 0.138$ \\
0.3277 & 24.55 & 0.8390 & 0.9776 & $0.758 \pm 1.071$ \\
0.3985 & 24.35 & 0.8996 & 0.9855 & $-0.269 \pm 0.461$ \\
0.4480 & 23.87 & 0.9265 & 0.9891 & $-5.002 \pm 23.776$ \\
0.2531 & 32.45 & 0.4220 & 0.9258 & $-0.003 \pm 0.137$ \\
0.3331 & 33.41 & 0.6766 & 0.9574 & $0.659 \pm 0.518$ \\
0.4106 & 32.91 & 0.8104 & 0.9739 & $0.610 \pm 1.164$ \\
0.4641 & 32.66 & 0.8603 & 0.9803 & $-4.844 \pm 11.585$ \\
0.5025 & $31.8: 3$ & 0.8904 & 0.9842 & $-1.857 \pm 0.975$ \\
0.3425 & 44.17 & 0.4131 & 0.9246 & $1.045 \pm 0.727$ \\
0.4145 & 44.10 & 0.6269 & 0.9512 & $1.557 \pm 1.732$ \\
0.4818 & 43.78 & 0.7436 & 0.9656 & $-4.027 \pm 3.908$ \\
0.5246 & 43.15 & 0.7975 & 0.9723 & $-4.764 \pm 11.744$ \\
0.5001 & 58.71 & 0.5274 & 0.9389 & $0.732 \pm 1.821$ \\
0.5528 & 57.96 & 0.6382 & 0.9526 & $-0.316 \pm 0.525$ \\
\hline 0.2278 & 22.80 & & & $-0.064 \pm 0.05 i$ \\
\hline
\end{tabular}

Table 10-VL $R$ vs. $Q^{2}, R$ rs. I

\begin{tabular}{|c|c|c|c|c|}
\hline $6^{2}\left(\mathrm{GeV}^{2} / c^{2}\right)$ & $R$ & $x$ & $R$ \\
\hline 18.2 & $-0.120 \pm 0.073$ & 0.194 & $-0.055 \pm 0.067$ \\
24.7 & $-0.004 \pm 0.130$ & 0.274 & $0.02 \pm 0.12$ \\
32.5 & $0.034 \pm 0.133$ & 0.392 & $-0.37 \pm 0.25$ \\
44.2 & $0.95 \pm 0.65$ & 0.541 & $-0.57 \pm 0.43$ \\
58.0 & $-0.27 \pm 0.47$ & & \\
\hline
\end{tabular}

that the average true $x$ and $Q^{2}$ can be different for the two besm energies due to differences in s mearing. energy loss, etc. To correct for this, we interpolate each measurement to the are:age $I_{\text {true }}$ and $Q_{\text {true }}^{2}$ of both messurements using the final $F_{2}$ fit for each energs. We eliminate measurements for which $\Delta I_{\text {rtue }}$ or $\Delta Q_{\text {true }}^{2}$ is greater than $10^{r}$ to minimize the sensitivity to this procedure.

The resulting measurements of $R$ are listed in Table 10-V and shown in Fig. 10.13. Points with uncertainties greater than 10 units of $R$ bave not been plotted. In Table 10-VI and Fig. 10.14 we show separately the dependence of $R$ on $Q^{2}$ and $I$. Because no strong dependence is apparent. 


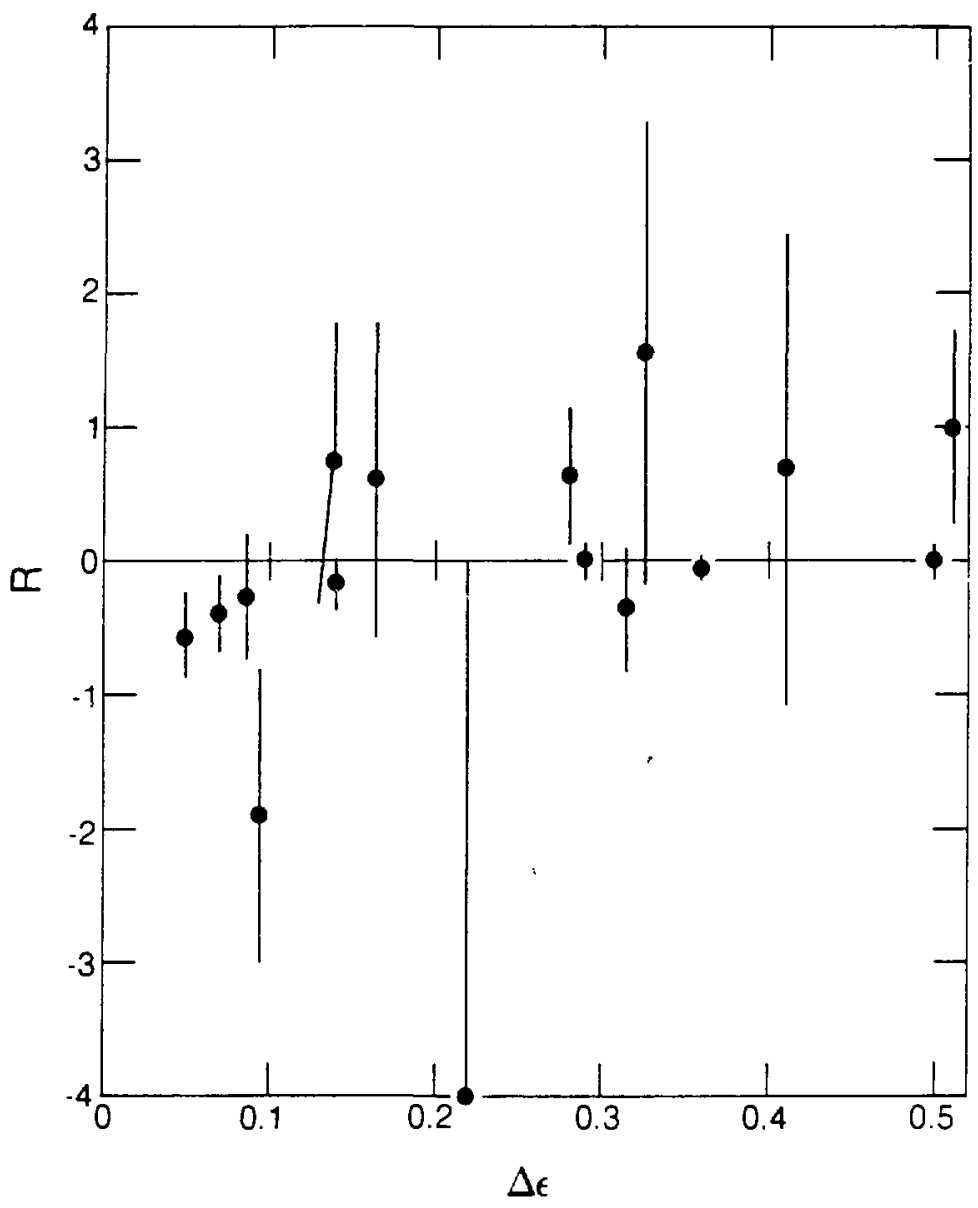

XBL 8310.723

Fig. 10.13. Measurements of $R$ from the comparison of our 93 and $215 \mathrm{GeV}$ data. All measurements it statistical uncertainties less than 10 are shown vs $\Delta \epsilon$. the difference in rirt ual photon polarization due to the different beam energies at each fliced $\left(r, Q^{2}\right)$. Errors are statistical. 

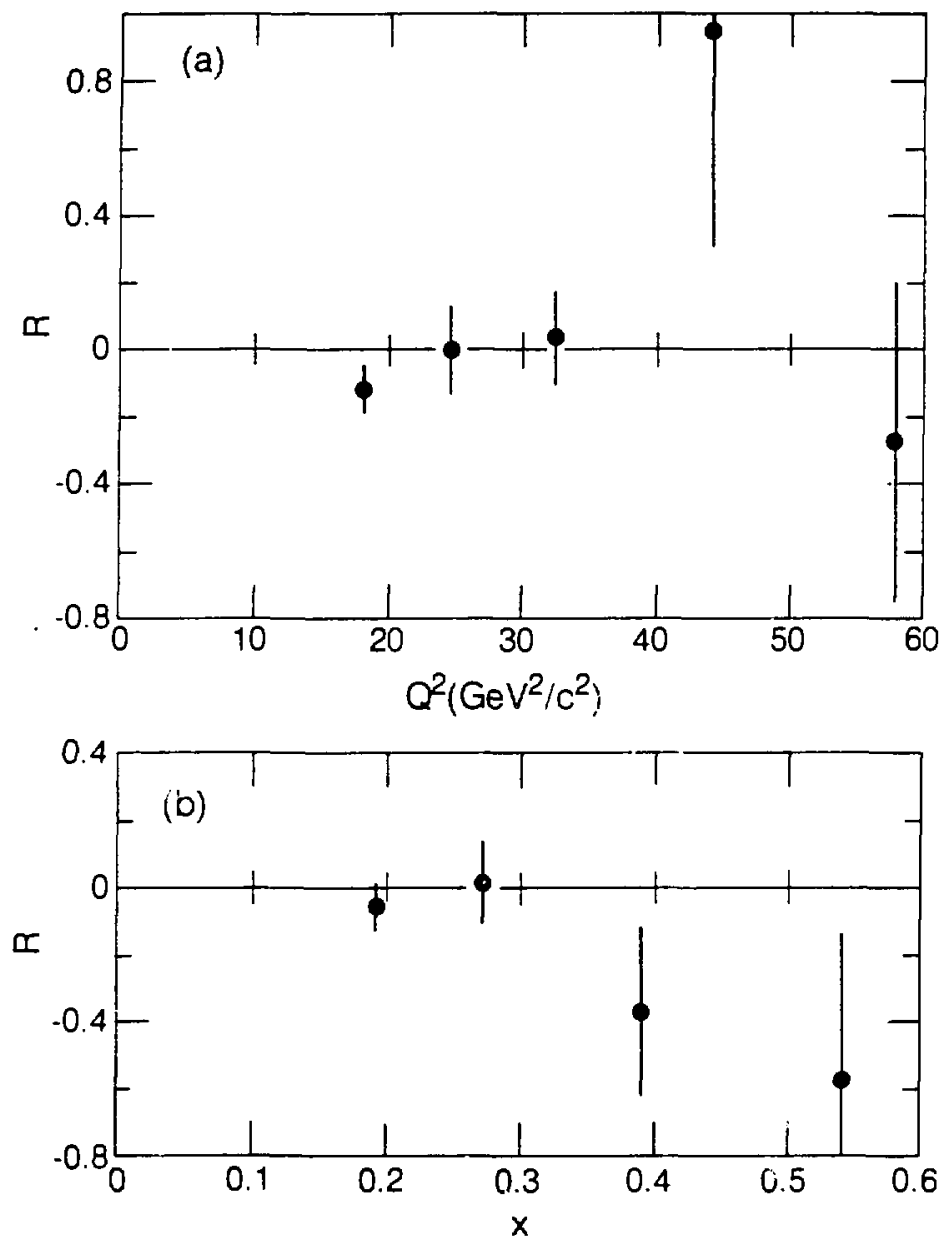

XBL 8310.739

Fig. 10.14. (a) $R$ ขs. $Q^{2}$; (b) $R$ vs. x. 
Table 10-VIL Systematic uncertainty in $R$. The total shors the sum of the other entries in quadrature.

\begin{tabular}{|c|c|c|}
\hline Source & L'ncerainty & $\delta R$ \\
\hline Mass B-Geld calibration & $0.5 r_{c}$ & 0.0049 \\
\hline Beam energy & $0.5 r_{c}$ & 0.0644 \\
\hline Trigger efficiency (systematic) & $0.5 \%$ & 0.0102 \\
\hline Trigger efficiency (statistical) & (see test) & 0.0018 \\
\hline Resolutis & (see text) & 0.0509 \\
\hline $93 / 215 \mathrm{GeV}$ normalization & $2.5 c_{c}$ & 0.0743 \\
\hline \multicolumn{2}{|l|}{ Total } & 0.1113 \\
\hline
\end{tabular}

we arerage all the measurements and find $R=-0.06$ with a statistical error of 0.06 . The kinematic range covered by the measurements is $20<\nu<70 \mathrm{GeV}^{2}$ and $18<Q^{2}<60 \mathrm{GeV}^{2} / \mathrm{C}^{2}$. with $\langle\nu\rangle=53 \mathrm{GeV}$ and $\left(Q^{2}\right\rangle=23 \mathrm{GeV}^{2} / c^{2}$. In Sec. 10.4 we observed the effect of rarying $R$ on our fitted results. This procedure can be used to infer a model dependent ralue of $R$. We find that. at fised normalization, the $x^{2}$ of our QCD fits is minimized for $R \approx 0.1$ (see Table $10-F v$ ).

The greatest contribution to the systematic uncertainty in $R$ comes from the uncertainty in the relative normalization of the $93 \mathrm{GeV}$ and $215 \mathrm{GeV}$ data sets. This uncertainty was estimated in Sec 9.2 to be $2.5 \%$. In Sec. 10.3, we calculated a model dependent correction to the relative normalization by including it as a variable in our QCD fit (xith $R=0$ ). The result was a -2.0 C correction to the $93 \mathrm{GeV}$ normalization. We can attempt a model- and $R$-independent Dormalization as suggested in Ref. 73 . by looking in the low $\nu$ region where both polarizations are near 1 . Unfort unately, if we restrict ourselves to $\epsilon_{1.2}>0.9$. we are left with only three points and a correction to the $93 \mathrm{GeV}$ normalization of $(-2.8 \pm 3.6)^{-} \mathrm{o}$. If we make these corrections. $R$ becomes $0.01 \pm 0.06$ or $0.04 \pm 0.06$ for the $-2.0 \sigma_{c}$ or $-2.8 \sigma_{c}$ corrections. respectively.

We also estimate the contributions to the systematic uncertainty in $R$ from the rest of the effects considered previously in fitting $F_{2}$. These uncertainties are shown in Table 10-vil. Included in the table is the contribution of a $2.5^{\circ}$ c relative normalization uncertainty. Our flnal result is $R=-0.06 \pm 0.06^{\text {stat }} \pm 0.11^{\text {syst }}$. For comparison, the EMC bas reported $R=0.00 \pm 0.10$ in muon-proton interactions for $60<\nu<160 \mathrm{GeV}$ and the same average $Q^{2}: 3$ Electron-nucleon scattering experimencs at $S L A C$ found $R=0.22 \pm 0.1$ at lower $Q^{2} .^{i t}$ Yeit her experiment observes any signibcant kinematic dependence in $R$.

\subsection{Search for a $\mu^{+-\mu^{-}}$asymnietry}

In considering only the radiatirely-corrected process of Figure $10.15 \mathrm{a}$, we bave neglected both the higher-order QED process of Fig. $10.15 \mathrm{~b}$ and the weak neutral current interaction of Fig. 10.15c. Alt hough too small to measure directly, these processes can give measurable effects through their interference with the one-photon exchange diagram. ${ }^{\text {is }}$ Weat interaction effects bave been observed in $e . V$ interactions in atomic physics ${ }^{-6}$ and in high energy $e . X^{*}$ interactions ${ }^{i T}$ by searching for a parity-riolating sigaal.

Recenty the BCDMS Collaboration at CER. reported an asymmetry bet ween the cross sections for $\mu^{+} . Y$ and $\mu-X$ interactions: ${ }^{79}$ 


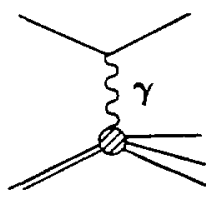

(a)

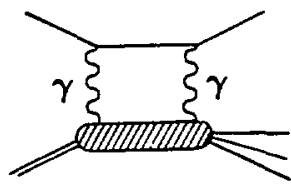

(b)

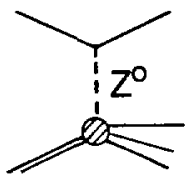

(c)

XBL 8310.734

Fig. 10.15. Contributions to deep inelastic scattering. (a) One photon exchange; (b) two photon exchange; (c) weak neutral current.

$$
A \equiv \frac{d \sigma_{L}^{+}-d \sigma_{\bar{R}}}{d \sigma_{L}^{+}+d \sigma_{\bar{R}}}
$$

The $L$ and $R$ refer to the predominant belicities of the $\mu^{+}$and $\mu-$ beams due to their production in the forward decay of pions. Because this as "mmetry violates neither parity nor charge conjugation, botb the two photon and weak neutral processes contribute. After correcting for the former, BCDMS finds an asymmetry consistent wit the Weinberg-Salam model prediction.

Athough all of our $93 \mathrm{GeV}$ data and nearly all of our $215 \mathrm{GeV}$ data was taken with a $\mu^{+}$beam. Table 5-1 shows that we do have a small amount of $\mu^{-}$data. While statistically insuffleient to probe the expected level of asymmetry (see below). the measurement bas value both as an exercise for the eager student and as a check of the systematic consistency of our data. It should be pointed out that a serious attempt to measure this asymmet ry would require more than additional $\mu$ - statistics. It would also demand better control of possible systematic differences. Although both the $\mu^{+}$and $\mu^{-}$data sets represent roughly equal amounts of east and west bending spectrometer settings, the $\mu$ - running was done all in one block and at a beam intensity of only about $\frac{1}{3}$ that of the $\mu^{+}$beam. Our Monte Carlo simulation takes into account known time-, intensity-, and beam phase space-dependent effects, but no attempt ras made to make these the same for the $\mu^{+}$and $\mu^{-}$running.

The expected asymmetry ${ }^{70}$ using the parton and Weinberg-Salam models is

$$
\begin{aligned}
A w S & =-4 \sin ^{2} \theta_{w}\left(\frac{9 G}{20 \sqrt{2} \pi a}\right) g(y) Q^{2}, \\
g(y) & =\frac{1-(1-y)^{2}}{1+(1-y)^{2}} .
\end{aligned}
$$

Here $\theta_{W}$ is the Weinberg angle, $G \approx 10^{-5} / M_{p}$ is the weak interaction coupling constant, and $y=\nu / E$. This approsimation assumes Bjorken scaling, an isoscalar target, and polarization of \pm 1 for the beams. It neglects Cabibbo mising and non-valence quarks. Because the currently accepted value of $\sin ^{2} \theta_{w} \approx 0.23$ is near $\frac{1}{3}$. the asymmetry is nearly independent of the actual polarization of the beams. The expected astmmetry is then $A$ ws $=-1.45 \times 10^{-4} g(y) Q^{2}$. The two-photon asymmetry is espected to be smaller and opposite in sign. ${ }^{79}$ 
We measure the asymmetry by extracting $F_{2}\left(r, Q^{2}\right)$ for the $\mu^{+}$and $\mu^{-}$samples separateis following the procedures described in Sec. 9.3. For each bin of $x$ and $Q^{2}$ for $w$ hich there is an $F_{2}$ measurement from both samples, the $\mu^{-}$measurement is interpolated to the average $I$ and $Q^{2}$ of the $\mu^{+}$measurement. We then calculate $g(y) Q^{2}$ and $A$ for each point. Figure 10.16 shows the asymmetry measurements after binning in $g(y) Q^{2}$. The line is the best fit of $A=a+b g(y) Q^{2}$ to all the points before binning. The result of this fit is $A=(6.9 \pm 6.7) \times 10^{-3}-(1.4 \pm 2.4) \times$ $10^{-4} g(y) Q^{2}$ with a $\chi^{2}$ of 48 for 44 degrees of freedom. As the statistical error in the slope $b$ indicates, setting $b=0$ has a negligible effect on $\chi^{2}$.

While, as expected, our lack of $\mu^{-}$statistics bars os from the realm of the weak interaction asymmetry, this measurement adds to the confidence we have in our ability to correct for systematic effects due to beam intensity and phase space. It also rules out an unexpectedly large contribution from the one photon-two photon interference term in muon scattering from iron that might affect the deuterium-iron comparison. Figure 10.17 shows the asymmetry us. I. There is no significant asymmetry at the $2 \%$ level out to $I=0.5$. 


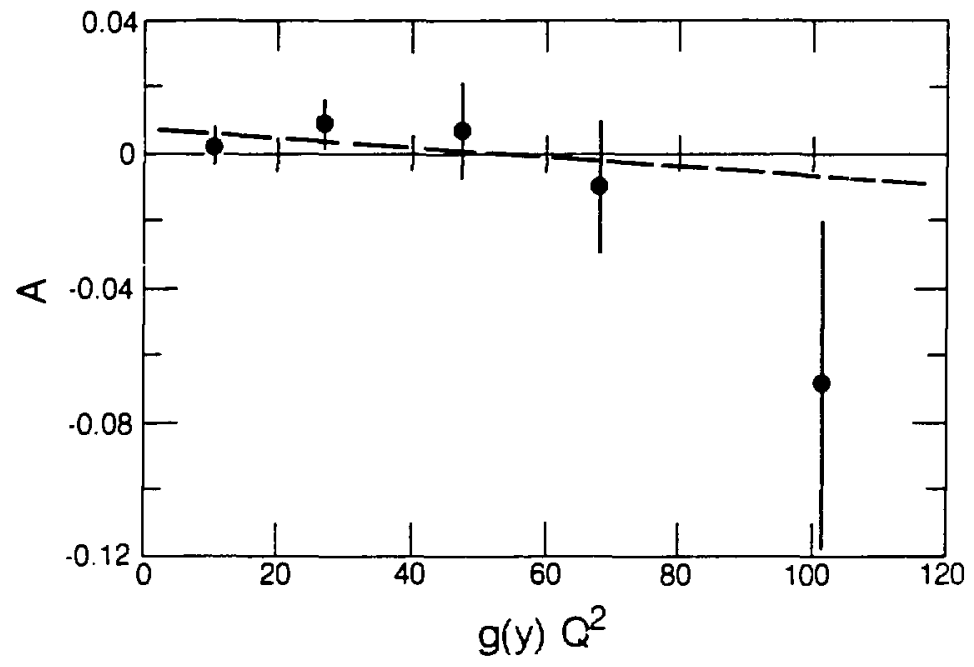

XBL 8310-729

Fig. 10.16. The $\mu^{+}-\mu^{-}$symmetry us. $g(y) Q^{2}$. The dashed line is a linear bit. Errors are statistical. 


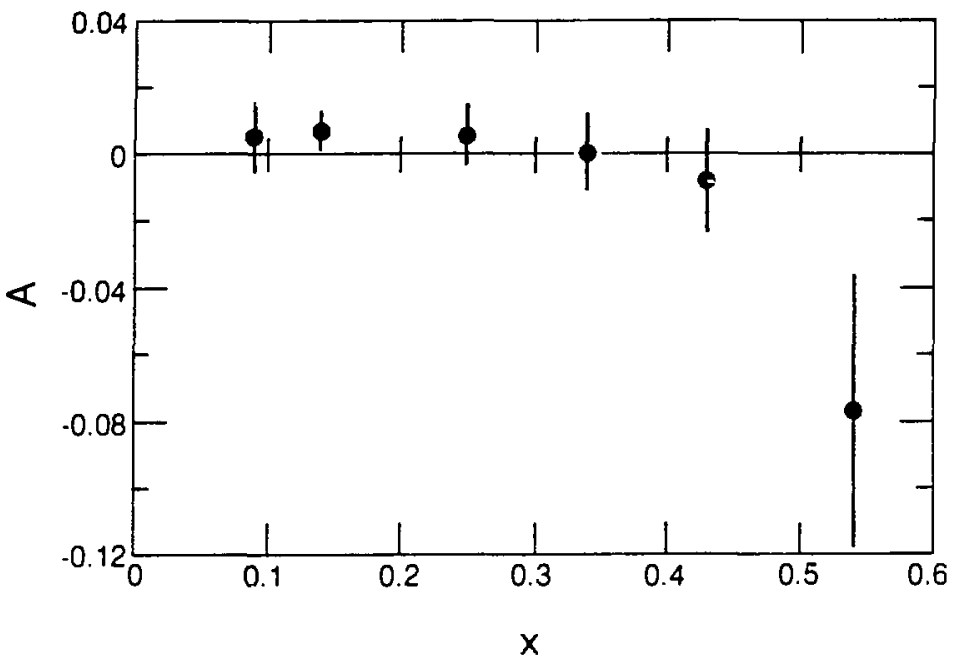

XBL 8310.730

Fis. 10.17. The $\mu^{+}-\mu^{-}$asymmetry vs. $x$. 


\section{Chapter 11 \\ Summary and Conclusions}

Bomp bomp bomp-BOMP BOMP. Bomp bomp bomp-BOMP BOMP.
Bomp bomp bomp-BOMP BOMP...

R. Berry, 1955

We hare built and operated the Maitimuon Spectrometer, an iron target-spectrometercalorimeter in the muon beam at Fermilab, to observe muon-nucleon interactions with high luminosity and broad acceptance. We have measured the cross section for the deep inelastic scattering of muons on nucleons in iron at incident energies of 93 and $215 \mathrm{GeV}$. Using the known properties of the electromagnetic interaction, we have extracted from the measured cross sections measurements of the structure function $F_{2}\left(x, Q^{2}\right)$ with a statistical precision of better than $2 \%$ over a broad kinematic range. These measurements, extending in $Q^{2}$ from 5 to $200 \mathrm{GeV}^{2} / c^{2}$, are presented in Table 10-I and in Fig. 10.2. Figure 11.1 illustrates the factors limiting the kinematic coverage of our experiment. Our measurements are in good agreement with results from similar contemporary experiments. The agreement in $x$ dependence between our data and that of the European M[uon Collaboration's iron target experiments is especially notable in the light of the controversy surrous jing the newly discovered $A$ dependence of $F_{2}(x)$.

We have compared our measurements to the predictions of lowest order quantum chromodynamics (QCD) in wich the $Q^{2}$ dependence of $F_{2}$ at flxed $x$ is calculable. $Q C D$ reproduces the qualitative pattern of scaling violation seen in the data. Within our set of phenomenological assumptions, xe measure the QCD sca!e parameter $\Lambda_{\text {Lo }}$ to be $230 \pm 10^{\text {stat }} \mathrm{MeV} / \mathrm{c}$. With the same assumptions, we estimate the systematic uncertainty in $A_{L O}$ to be $80 \mathrm{MeV} / \mathrm{c}$. This value of $A$ agrees within quoted errors with the determinations from other deep inelastic scatte.ing experiments using muons and neutrinos. While the statistical precision and $Q^{2}$ range of the Lew experiments represent an improvement over those of several years ago, systematic uncertainties still limit the precision with which we measure $\mathrm{A}$ to about $100 \mathrm{MeV} / \mathrm{c}$. If we relas some of our assumptions about imprecisely known parameters, we can again generate $\approx 100 \mathrm{MeV} / \mathrm{c}$ uncertainties in $\mathrm{A}$. These uncerainties will diminish as the quantities in question, notably $R$ and the shape of the gluon spectrum, become better known, but progress in this direction has been slow and diticult. We have compared our $F_{2}$ measurements from the 93 and $215 \mathrm{GeV}$ beam energies and measured $R=-0.06 \pm 0.06^{\text {stat }} \pm 0.11^{\text {synt }}$, a value consistent with zero, but with enough uncertainty to affect 1 substantially. Similarly, the rejection of large contributions to the observed scaling riolations from $1 / Q^{2}$ terms in favor of the logarithmic behavior predicted by $Q C D$ is diffcult. especially with little constraint on the form of such terms. Athough the confirmation from deep inelastic scattering of QCD as the theory of the strong interactions must 


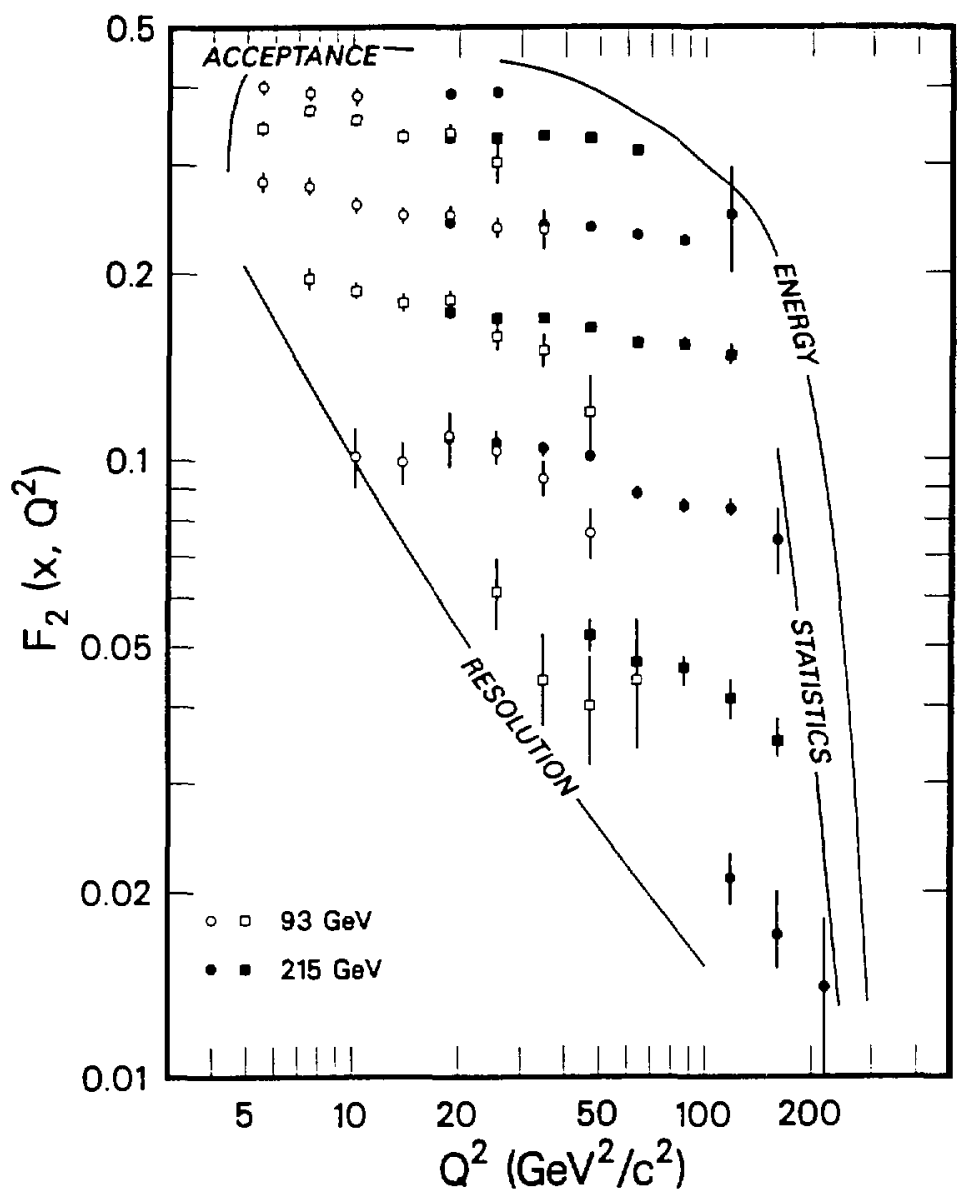

XCG 8311-4902A

Fig. 11.1. The various factors limiting the kinematic range of our measurement of $F_{2}\left(x, Q^{2}\right)$. 
still be considered somewhat qualjtatjwe, the improved agreement between the rarious experiments represents a distinct clarifleation of the experimental situation, without which no progress can be possible. 


\section{Appendix A \\ Radiative Corrections}

As snmmarized in Chapter 8 , we correct the deep inelastic scattering cross section Eq. 8.5 for processes involving the radiation of photons (Fig. 8.2a-d). We do this by asing the method of equivalent radiators ${ }^{32}$ in which these internal bremsstrahlung diagrams are replaced by an equiralent amount of radiation emitted separately trom the primary (deep inelastic) scatter. The connection to the primary scatter remains in the amount of radiation. which is effectively that due to a $Q^{2}$ dependent number of radiation lengt hs

$$
t=\frac{3 \alpha}{4 \pi}\left[\ln \left(Q^{2} / m_{\mu}^{2}\right)-1\right]
$$

This radiation has the characteristic spectrum

$$
P(y) d y=\left(1-y+\frac{3}{4} y^{2}\right) \frac{d y}{y}
$$

where $y=\left|E-E^{\prime}\right| / E$ is the muon's fractional energy loss in the radiator. We radiatively correct the cross section in our event simulation by explicitly radiating a photon before the interaction with a probability and spectrum given by Eqs. $A .1$ and 2. Since the $Q^{2}$ of the interaction is not known before the interaction, we use a large lim.ing nue, to be corrected later. The divergent spectrum is cut off at $y_{m, n}=0.001$. We invole the peaking approximation, in wich the muon's direction is unaffected by the photon cmission, and the incident muon, wit b its energy degraded. is handed to the deep inelastic generator. Besides the energy loss due to the process of Fig. 8.2a, the cross section is modified by the processes of Fig. 8.2b and c. The vertex and racuum polarization corrections (Fig. 8.2b) have the effect of multiplying the cross section by ${ }^{52}$

$$
1+\delta\left(Q^{2}\right)=1+\frac{2 \alpha}{\pi}\left[-\frac{14}{9}+\frac{13}{12} \ln \left(Q^{2} / m_{\mu}^{2}\right)\right] .
$$

We rogenerate the wide angle bremsstrahlung (WAB) events of Fig $8.2 \mathrm{c}$ using the per nucleon cross section ${ }^{\text {so }}$

$$
\frac{d^{2} \sigma^{W+B}}{d v \cdot d y}=\frac{1}{A} \frac{4 Z^{2} \alpha^{3}}{2 M N E} \frac{y\left(1-y+y^{2} / 2\right)}{(1-y) l^{2}} G\left(Q_{11}^{2}\right)
$$

xhere $G\left(Q_{\|}^{2}\right)$ is the nuclear form factor integrated over the component of $q$ perpendicular to the incident muon direction. Finally. photon emission from the outgoing muon is simulated. The artual $Q^{-}$of the event is then calculated and excess radiation is remored. 
To investigate the effects of the radiative corrections, I have convolved numerically the deep inelastic seattering cross section with the radiative effects of Eqs. A.1-3. The cit off at $y_{\mathrm{m}} \mathrm{m}$ is imposed by calculating the total probability of the emission of a photon from a single radiat or

$$
k\left(y_{m \times n}, Q^{2}\right)=\frac{\alpha}{\pi}\left[\ln i\left(Q^{2} / m_{s s}^{2}\right)-1\right] \int_{y=m}^{1} P(y) d y .
$$

The convolution then breaks into four terms:

1. an "unradiated" term with weight $\left[1-\delta\left(Q^{2}\right)\right]\left[1-k\left(y_{m, n}, Q^{2}\right)\right]^{2}$.

2. an "initial radiator only" term with weight $\left[1-k\left(y_{m, n}, Q^{2}\right)\right]$ (the probability of no final emission greater than $y_{\min }$ ).

3. a "final radiator only" term with weight $\left[1-k\left(y_{m i n}, Q^{2}\right)_{j}\right.$.

4. a "both radiators" term with weight 1 .

As a point of reference, for $y_{\min }=0.001$, the probability of photon emission rith $y>y_{\min }$ in a single radiator is 0.082 at $Q^{2}=10$ and 0.125 at $Q^{2}=100 \mathrm{GeV}^{2} / c^{2}$.

The results of this calculation are shown in Fig. A.I for a beam energy of $200 \mathrm{GeV}$ and the same $F_{2}\left(\tau, Q^{2}\right)$ used in the event simulation routine. 5 Ploted is $d \sigma^{\text {cort }} / d \sigma^{\text {uncorr }}$ as a function of $Q^{2}$ for ised $x$. For a flxed observed cross section, $d \sigma^{\text {corr }} / d \sigma^{\text {uncorr }}>1$ corresponds to a downward correction in measured $F_{2}$. Changing the beam energy to $100 \mathrm{GeV}$ has an effect very similar to sliding the plot down by a factor of two in $Q^{2}$. Altbougb it was not included in our event simulation, in this calculation I also studied the contribution of the radiative tail from elastic scattering. This correction turns out to be invisible where we have data. reaching a maximum of $<1{ }^{c} c$ at the highest $Q^{2}$, lowest $I$ point of the $93 \mathrm{GeV}$ data.

After the cuts restricting the region ower which we present $F_{2}\left(x, Q^{2}\right)$ are applied. the contribution of $W A B$ is never greater than $3 \%$. The number of simulated WAB events found in each bin of measured $x$ and $Q^{2}$ is subtracted from bot $h$ the real and simulated events before the $F_{2}$ extractiun procedure of Section 9.3. The iteration of the $F F^{\text {eq }}$ used in the simulation does not modify the WAB events.

As an order of magnitude check on the presence of radiative events, I looked for the difference between hadronic and electromagnetic showers in the calorimeter. In Fig. 7.2 we see evidence in the caiorimeter calibration for the expected electromagnetic trents at low $Q^{2}$. For a given energy, electromagnetic showers are shorter than hadronic showers. Looking at a sample of deep inclastic events with $y>0.6 i$, but short showers, showed them to occur at roughly the same rate as simulated $W A B$ events and in bave similar properties. 


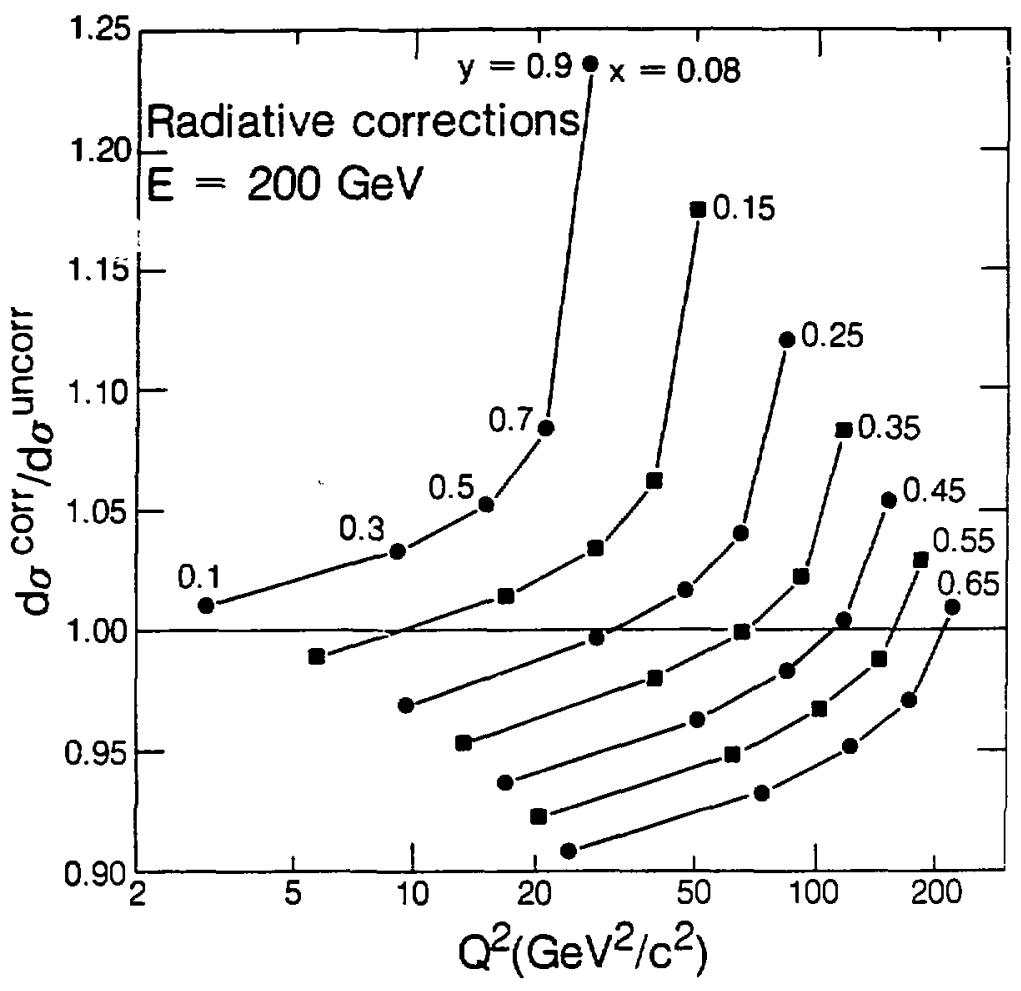

XBL $83 \div 1.768$

Fig. A.1. The effect of radiative corrections on the deep inelastic seattering cross section. 


\section{Appendir B \\ Fermi Motion}

In analyzing our data we hare calculated the rarious kinematic quantities using Eqs. 2.1 and thus have assumed that the laboratory frame and the target rest frame were the same. Ir our experiment, this is fine if the target is considered to be an iron nucleus, as in most of our results where we have presented $F_{2}^{\mathrm{fe}} / 56$. In order to interpret our results in terms of $F_{2}$ for a single nucleon. we must remove the effects of nuclear binding, beginning with Fermi motion. Athough the energy scale of Fermi motion seems negligible compared to that of our $200 \mathrm{GeV}$ muons, a proton with a Fermi momentum of $240 \mathrm{MeV} / c$ can change the effectire beam energy by orer $50 \mathrm{GeV}$. The treatment of Fermi motion and binding can be broken into three nearly independent phases. The first is the determination of the momentum spectrum of the target nucleons. The second is the treatment of binding energy. The third is the calculation of the effect on the cross section, and thus on the measurement of $F_{2}$.

The nucleon momentum spectrum we use is derived from measurements of the nuclear form factor. ${ }^{91}$ augmented by a tail inferred from studies of antiproton production below threshold. ${ }^{\$ 2}$ A zero-temperature, spherically symmetric Fermi gas has a kinetic energy (T) spectrum proportional to $T^{1 / 2}$ below the Fermi energy $T_{f}$. Our spertrum has a form corresponding to a finite temperature plus the tail:

$$
\frac{d N}{d T} \sim \begin{cases}T^{1 / 2} /\left(1+e^{(T-38) / 0.4}\right), & T<70 \mathrm{MeV}: \\ T^{-2.6}, & 70<T<385 \mathrm{MeV} \\ 0, & T>385 \mathrm{MeV}\end{cases}
$$

This spectrum as a function of nucleon momentum is shown as the solid curre in Fig. B.1. Also shown in the foure is the spectrum used by Bodek and Ritchie. ${ }^{83}$ The interpretation of the tails in these spectra is not entirely clear. Rather than single high energy nucleons. the tail may represent a collectire effect in the nucleus, such as the scattering off a higher mass cluster of nucleons.

The treatment of binding is, in effect, the calculation of the energy component of the target nucleon's 4 -momentum. The various models thus provide a function $E(p)$ giving the energy for any momentum cbosen from the spectrum of the previous paragraph. I will consider tro such models here. The first is an independent particle model - each nucleon in the potential well formed by : he others. ${ }^{\text {S4 }}$ We derive the parameters of this well from the known static properties of nuclei. From the table of nuclides we can deflne the separation energy $S$ needed to remove 


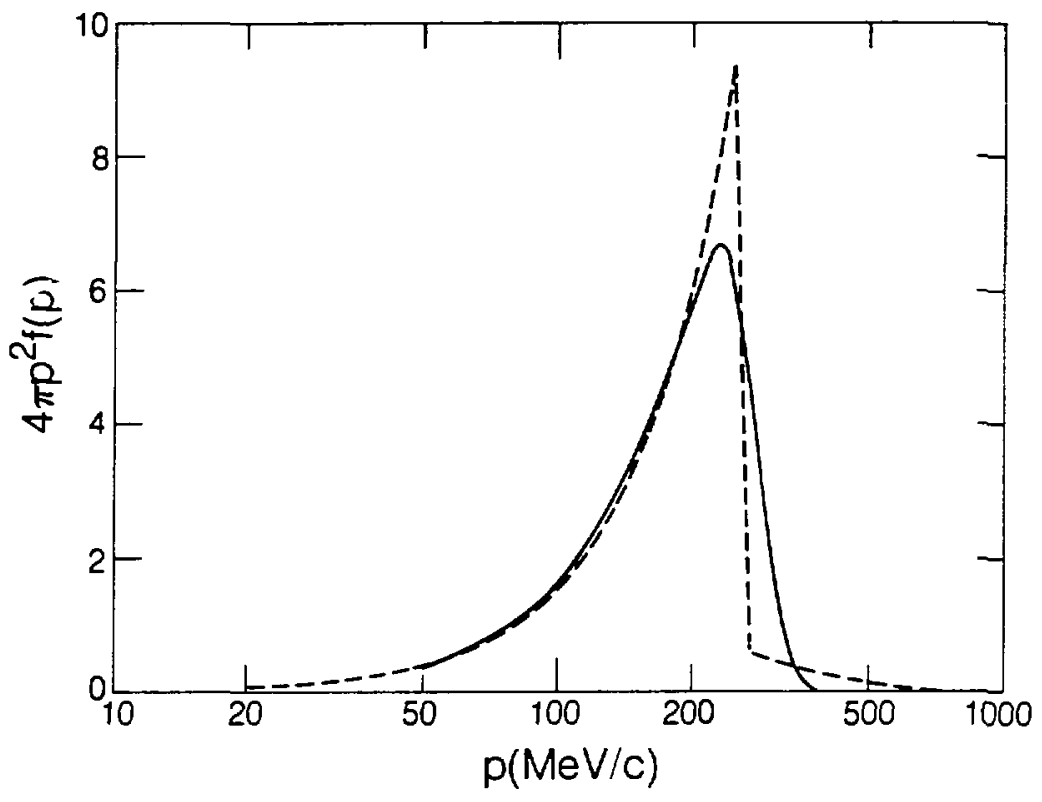

x马L $8311-762$

Fig. B.1. Fermi momentum spectrum of nucleons in iron (solid curve). Also shown is the spectrum used in Ref. 83. (dasbed curve). 
the most weakly bound nucleon (at zero temperature), and the binding energr per nucleon $B / A$

$$
\begin{aligned}
S & =M(A, Z)-M(A-1, Z)-M_{n}=V+T_{f} . \\
B / A & =\left(M(A, Z)-Z M_{p}-(A-Z) M_{n}\right) / A=\frac{1}{2}(D)+(T) .
\end{aligned}
$$

where $V$ is the potential and the $\frac{1}{2}$ is necessary to avoid double counting since the potential seen by each nucleon is actually due to all the otbers. In the limit of "nuclear matter," that is. a collection of nucleons of arbitrary extent, $S=B / A$. and Weisskopf ${ }^{55}$ bas show that a consistent picture demands a velocity dependent potential $v(T)$. For a linear $v^{\prime}(T)=v_{0}+Q T$ we can solve for $V_{0}$ and $a$ and thus determine $E(T)=M_{N}+V_{0}+(1+a) T$.

Bodek and Ritchie use an entirely different model which can be described as the spectat or or coberent recoil picture. In their model, the target nucleon is required to conserve energr and momentum by recoiling against an on-shell spectator nucleus with mass number $A-1$. Thus the moment um of the spectator nucleus is equal and opposite to that of the target nucleon and $E=M_{A}-E_{\text {spectator }}=M_{A}-\left(p^{2}+M_{A-1}{ }^{2}\right)^{1 / 2} \approx M_{N}+S-\left(M M_{N} / M_{A-1}\right) T$. Sote that in this case. the nucleon's energy decreases slowly as its momentam increases. The two models are show $\mathrm{n}$ in Fig. B.2 for iron $\left(T_{f} \approx 36 \mathrm{MeV}, S \approx-11 \mathrm{MeV}, B / A \approx-8 \mathrm{MeV}\right)$. Each of these models bas advantages and disadvantages. The independent particle model arbitrarily invokes the existence of nuclear matter and the linear form of $V(T)$. The spectator model aribitrarily assumes the recoil of a real, on-shell nuclens of mass number $A-1$ (although Bodek and Ritchie modify this for $T>T_{f}$ ). I have been unable to choose between them, and have investigated the effects of each.

To apply these Fermi motion models to deep inelastic scattering. we follow the work of West. $^{96}$ The starting point is the impulse approximation $: 0$ which the complicated interactions within the nucleus are considered to affect things only by detrrmining the wave function of the target nucleon, that is, the momentum spectrum and $E(p)$. Hence

$$
W_{\mu \nu}^{A}\left(P^{A}, q\right)=A \int_{0}^{\infty} 4 \pi p^{2} d p f(p) W_{\mu \nu}^{N}(p, q)
$$

where ${ }_{\mu \nu}$ is tbe hadron tensor introduced in Eq. 2.4 and $A$ and $N$ refer to the nucleus and nucleon. Csing Eq. 2.6 and inspecting the 3-3 component of Eq. B.3 we flad

$$
W_{2}^{A}\left(P^{-4} \cdot q\right)=A \int_{0}^{\infty} 4 \pi p^{2} d p f^{\prime}(p)\left[\left(1+\frac{p_{3} Q^{2}}{M_{N} \nu^{\prime} q_{3}}\right)^{2}\left(\frac{\nu^{\prime}}{\nu}\right)^{2}+\frac{p_{\perp}^{2} Q^{2}}{M_{2}^{2} \cdot q_{3}^{2}}\right] W_{2}^{N}(p, q)
$$

where we have chosen the 3 -axis along the direction of $\mathrm{q}$, and $w^{\prime} \equiv p \cdot q / h_{N}, \nu \equiv P^{A} \cdot q / . k_{j_{i}}$. The remaining diffculty is that $W_{2}^{N}$ in Eq. B. 4 still refers to the structure function of an off-shell nucleon. That this might complicate the situation is evident. Our derisation of $w_{\nu \nu}$ was based on the presence of only two relevant scalars. Now, with $p^{2} \neq M_{3}^{2}, W_{2}^{-N}$ can be a function not only of $Q^{2}$ and $\nu^{\prime}$. but also $p^{2}$. Following Bodek and Richie, we identify the of-shria $\{(p, q)$ with the on-shell rersion at the same $Q^{2}$ and invariant mass $\mathfrak{l}^{2}$. Hence

$$
\begin{aligned}
W_{2}^{N, \text { ofl-shell }}(p, q) & =F_{2}^{N}\left(x^{\prime}, Q^{2}\right) / \nu . \\
Q^{2} & =-q^{2} . \\
x^{\prime} & =\frac{Q^{2}}{2 M, \nu^{\prime}+p^{2}-M^{2}} .
\end{aligned}
$$




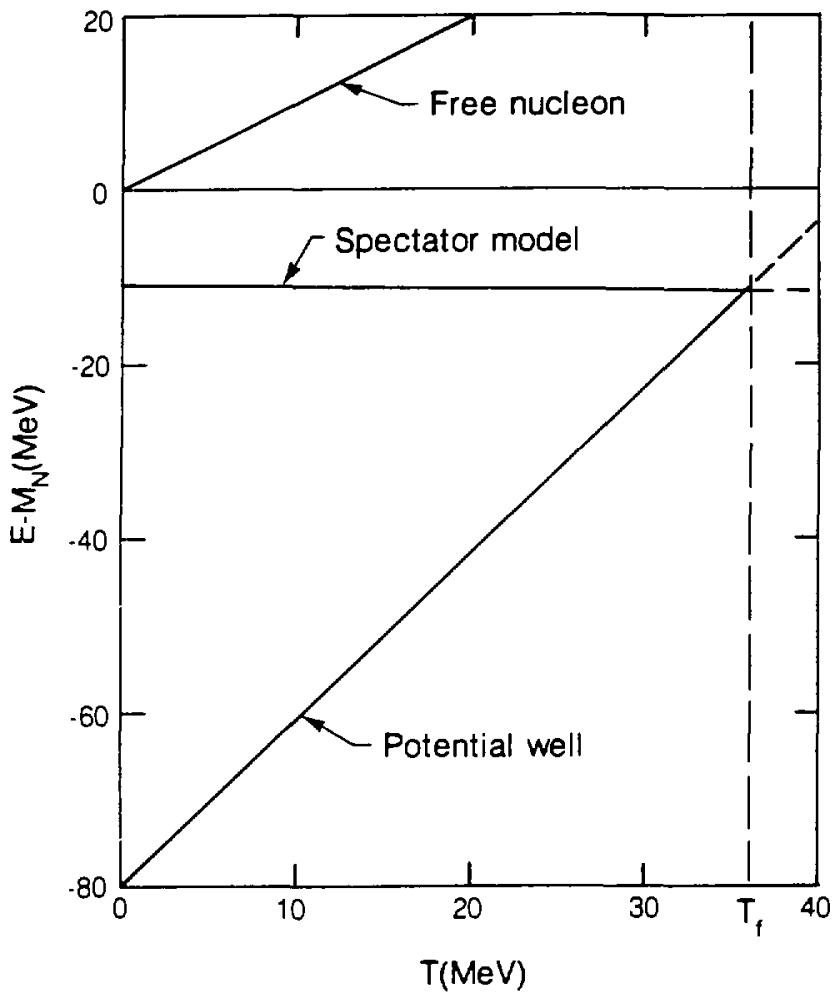

XBL $8311-761$

F.6. B.2. The effects of binding. Total energy us. kinetic energy is sbown for a free nucleon and for the two nuclear models of iron discusse $f$ in the text. 
Where $F_{\dot{2}}^{x}$ is the standard on-shell structure function.

With $f(p), E(p)$, and an assumed $F_{2}^{N}\left(x, Q^{2}\right)$, one can perform the integral in Eq. ? numerically and calculate the Fermi motion correction defined as $F_{2}^{i}\left(x, Q^{2}\right) /\left(\frac{1}{5 b} F_{2}^{\mathrm{Fe}}\left(x, Q^{2}\right)\right)$. The results of such a calculation are shown in Fig. B.3 for the independent particle model and our spectrum. For comparison, the dashed curves are the results of Bodek and Ritchie using the spectator model. Both calculations used the Buras-Gaemers parametrization of $F_{2}^{*}$ as input ${ }^{51}$ The two calculations agree fairly well in $Q^{2}$ dependence, which is minor below $r=0.45$. However. the $I$ dependence differs substantially abore $x=0.4$. It should be noted that a calculation $w$ hich neglects the effects of binding entirely, that is, with $E(p)=\sqrt{\left(p^{2}+M_{i}^{2}\right)}$, yields a correction with a similar shape to those shown, but which is everywhere less than one. Applying these corrections to our measured $F_{2}^{F e}\left(x, Q^{2}\right) / 56$ before fitting resulted in the changes noted in the text. The independent particle model yielded the smaller correction to $\mathrm{A}$. alt hough others have found corrections nearly as small using the model of Bodek and Ritcbie. ${ }^{\text {at }}$ 


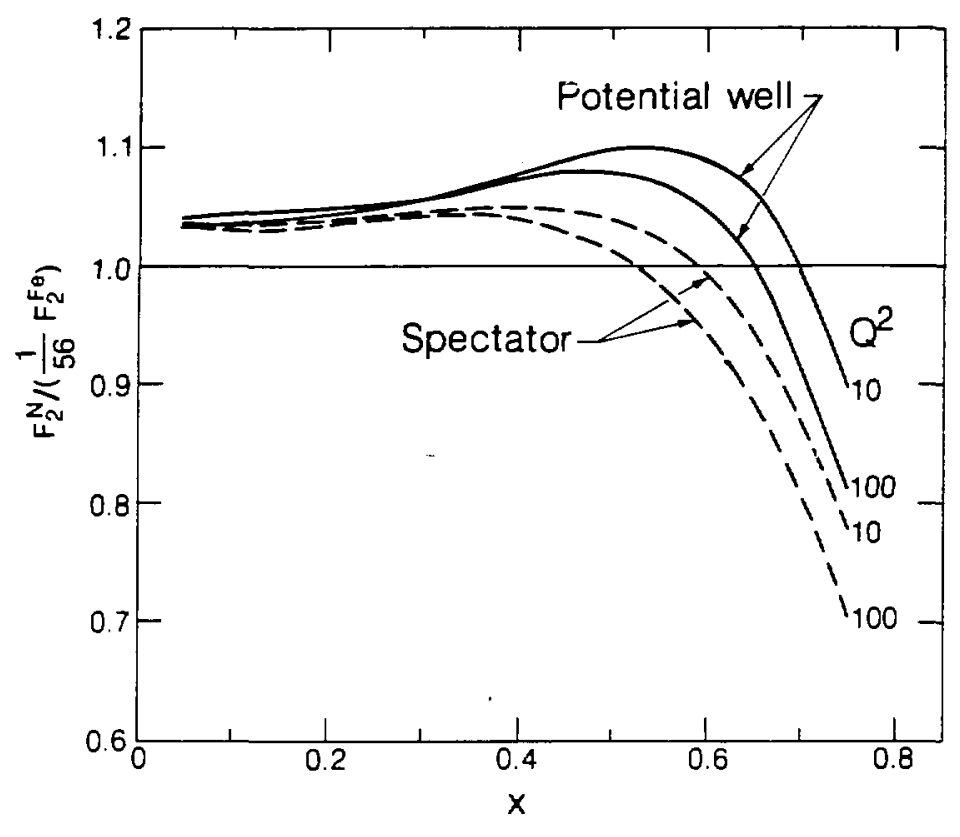

X51 83,960

Fig. B.3. The effects of Fermi motion on $F_{2}$. The two noclear models discussed in the text have been used to calculate $F_{2}^{\mathrm{Fe}}$ given $F_{2}^{*}$. The result is shown as a function of $x$ for $Q^{2}=10$ and $100 \mathrm{GeV}^{-2} / \mathrm{c}^{2}$. The dashed curves are the results of Ref. 83. 


\section{References}

1. J.J. Thomson, Philos. Mag., Ser. V 48, 547 (1899).

2. J.J. Thomson, Camb. Lit. Philos. Soc. 15, 465 (1910).

3. H. Geiger and E. Marsden, Proc. R. Soc. London, Ser. A 82, 495 (1909).

4. E. Rutherford, Philos. Mag., Ser. VT 21, 669 (1911).

5. H. Geiger and E. Marsden, Philos. Mag., Ser. VI 25, 604 (19l3).

6. R. Hofstadter, Ann. Rev. Nucl. Sci. T, 231 (195i).

7. W.K.H. Panofsky, in Proceedings of the Heidelberg International Conference on Elementary Particles, edited by H. Filthuth (North-Holland Publishing Co.. Eeidelberg, Germany, 1967), p. 371.

8. E.D. Bloom et a... Phys. Rev. Lett. 23, 930 (1969); M. Brejdenbach et a.., Phys. Rev. Lett. 23. 935 (1969).

9. J.L. Bjorken and E.A. Paschos, Phys. Rev. 185. 1975 (1969).

10. J.D. Bjorken, Phys. Rev. 179, 1547 (1969).

11. M. Gell-Mann. Phys. Lett. 8. 214 (1964).

12. G. Zweig, CERN Reports TH-412 (1964) (unpublished).

13. H.D. Polizzer, Phys. Rep. 14, 129 (1974).

14. D.J. Fos et al., Phys, Rev. Lett. 33. 1504 (1974): C. Chang et al, Phys. Rev. Lett. 35, 901 (1975).

15. A. Bodek et al., Phys. Rev. D20, 1471 (1979).

16. B.A. Gordon et ad., Phys. Rev. D20, 2645 (1979).

17. J.D. Bjorken and S.D. Drell. Relativistic Quantum Mechanics, (McGraw Bill Book Company, Yew York, 1964).

18. S.D. Drell and J.D. Walecks. Ann. Phrs. (X.Y.) 28. 18 (1964).

13. L.X. Hand, Phys. Rev. 129, 1834 (1963).

20. C.G. Callen and D.J. Gross. Phys. Rer. Lett. 22, 156 (1969).

21. F.E. Close, An Introduction to Quarks and Partors. (Academic Press, London. 1979), p. 221. 
22. M. Gell-Mann and Y. Ye eman, The Eightfoid Way, (W.A. Benjamin. Inc., Nen York, 1964).

23. This heuristic approach is derived from a seminar given by I. Hinchliffe, Lawrence Berkeley Laboratory (1979).

24. Newsweek Magazine 30,60 (Sept. 29. 194i). It was from this article that Tomonaga and other Japanese physicists learned of the discorery of the Lamb shift. See $Y$. Nambu and K. Nishijima. Enrico Fermi Institute Preprint EFI 83/34. June 1983 (unpublished).

25. D.J. Gross and F. Wilczek, Phys. Rer. Lett. 30, 1343 (1973); H.D. Politzer, Phys. Rer. Lett. 30, 1346 (19i3).

26. M. Bace, Phys. Lett. 78B, 132 (1978).

27. G. Altarelli and G. Parisi, Nucl. Phys. B126, 298 (1977).

28. F.J. Yndurain, Quantum Chromodynamics: An Introduction to the Theory of Quarks and Gluons, (Springer-Verlag, New York, 1983).

29. H. Abramowicz et al., Z. Phy̧s. C12, 289 (1982).

30. T. Markiewicz, Lniversity of California Ph.D. Thesis, (Berkeley, 1981).

31. S.L. Glasbor. Nucl. Phys. 22. 579 (1961); A. Salam and J.C. Ward. Pbys. Letr. 13, I68 (1964): S. Weinberg, Phys. Rer. Lett. 19, 1264 (1967).

32. P. Langacker, Phys. Rep. 72, 185 (1981).

33. H. Georgi and S.L. Glashow, Phys. Rev. Let.t. 32. 438 (19-4).

34. A.D. Sakharor, JETP Lett. 5, 24 (1967).

35. A.H. Guth, Center for Theoretical Physics (M.I.T.) Preprint CTP-1050 (Jan. 1983). to be published in the Proceedings of the XI Tesas Symposium on Relativistic Astrophysics (Austin, Tezas, 1982).

36. M. Goldhaber, P. Langacker, and R. Slansky, Science 210, 851 (1980).

37. R.M. Bionta et al, Phys. Rev, Lett. 51, 27 (1983).

38. G.D. Gollin et ad., Phys. Rev. D24, 559 (1981).

39. O.C. Allkofer et ad., Nucl. Instrum. Merhods 179, 445 (1981).

40. D. Nelson, Lawrence Berkeley Laboratory Engineering Note MT-260. May 24, 1977 (unpublished).

41. D. Erans. Kerth Group Internal Note GLV-65, Lamrence Berkeley Laboratory, June 15, 1978 (unpublished).

42. F. Ratliff. Sci. Am. 226. 90 (June,1972).

43. E. Mach, Sitzber. Akad. Wiss. Wien Math. Naturm. Kl.U 52, 303 (1865).

44. G. Gollin et al, IEEE Trans. Nuc. Sci. NS-26. 59 (1979).

45. B. Barish et al. in Proceedings of the Colorimeter Workshop, edited by M. Atac. (Fermilab. May 1975), p. 229.

46. A.R. Clark et ad. Pbys. Rev. Lett. 43. 187 (1979).

4i. A.R. Clark et ad., Phys. Rev. Lett. 45, 682 (1980). 
48. A.R. Clark tt al, Phys. Rev. Lett. 45, 686 (1980).

59. A.R. Clark et al., Phys. Rev. Lett. 45, 1465 (1980).

50. M. Stravink, Kertb Group Internal Note GN-100, Lamrence Berkeley Laboratory, December 18, 1978 (unpublisbed).

51. A.J. Buras and K.J.F. Gaemers, Nucl. Phys. B132, $249(19 ; 8)$.

52. L.W. Mo and Y.S. Tsai, Rer. Mod. Phys. 41, 205 (1969).

53. R.H. Siemann et al.. Phys. Rer. Lett. 22, 421 (1969).

54. F.C. Shoemaker, Kerth Group Internal Totes GLY-153, April 7, 1980 and GL-155. April 9, 1980 (unpublished).

55. G. Molière, Z. Natuforsch. 3a, 78 (1948); H.A. Bethe, Pbys. Rev. 89, 1256 (1953).

56. B.R. Frieden, "Image Enhancement and Restoration" in Picture Processing and Digital Filtering, Topics in Applied Pbysics, v.6, edited by T.S. Huang, (SpringerVerlag, New York, 1975), p. 177.

5i. L.F. Abbott, W.B. Atwood, and R.M. Barnett, Pbys. Rev. D22, 582 (1980).

58. F. James and M. Roos, Computer Phys. Comm. 10, 343 (19i5).

59. D.J. Gross and C.H. Liewellyn Smith,Nucl. Phys. B14. 337 (1969).

60. R.M. Barnett, Phys. Rev. Lett. 48, 1657 (1982).

61. J.J. Aubert et al. Phys. Lett. 123B, 275 (1983).

62. A. Bodek et al. Pbys. Rev. Lett. 51, 534 (1983).

63. R.M. Barnett and D. Schlatter, Pbys. Lett. 112B. 475 (1982).

64. A. Devoto et al. Phys. Rev. D27, 508 (1983).

65. J.J. Aubert et al., Pbys. Lett. 114B, 291 (1982).

66. H. Georgi and H.D. Politzer, Phys. Rev. D14, 1829 (19/6).

67. J.J. Aubert et al. Phys. Letc. 105B, 322 (1981).

68. H. Abramonicz et al., Z. Phys. C17, 283 (1983).

69. H.E. Fisk and F. Sciulli, Ann. Rev. Nucl. Part. Sci. 32, 499 (1982).

70. S. Wu. in Proceedings of the 198R SLAC Summer Institute on Particle Physics, (SLAC, Stanford. CA, 1982). and references therein.

i1. P.B. Mackeazie and G.P. Lepage, Phys. Rev. Lett. 17, 1244 (1981).

i2. F.J. Gilman, Phys. Rev, 167, 1365 (1968).

73. J.J. Aubert et al, Phys. Lett. 1218, 87 (1983).

it. M.D. Mestayer et al., Phys. Rev. D27, 285 (1983).

75. E. Derman, Phys. Rev. D7, 2755 (1973).

i6. E. Commins and P. Bucksbaum. Weak Interactions of Leptors and Quarks. ICambridge Liniversity Press, Cambridge, 1983), pp. 343-52.

77. C.Y. Prescott et al. Phys. Lett. 77B. 347 (1978): 84B, 524 (1979).

78. A. Argento et al. Phys. Lett. 120B. 245 (1983). 
79. S.M. Berman and J.R. Primack, Phys. Rev. D9. 2171 (1974); D10, 3895(E) (1974).

80. M. Strovink, internal memorandum. July 22, 1974 (unpublished).

81. B. Hahn, D.G. Ravenhall, and R. Hofstadter, Pbys. Rev. 101, 1131 (1956).

82. D.E. Dorfan et al, Phys. Rev. Let.t. 14, 995 (1965).

83. A. Bodek and J.L. Ritchie, Phys. Rev. D23, 1070 (1981); D24, 1400 (1981).

84. A. Bohr and B.R. Mottelson, Nuclear Structure, v.I. (W.A. Benjamin. Iac.. New York, 1969), p. 146.

85. V.F. Weisskopf, Nucl. Phys. 3, 423 (195i).

86. G.B. West, Ann. Phys. (N.Y.) 74, 464 (1972). 